Supporting Information

\title{
Catalytic Asymmetric Synthesis of Vicinal Tetrasubstituted Diamines via Umpolung Cross-Mannich Reaction of Cyclic Ketimines
}

Wen-Run Zhu, ${ }^{\dagger}$ Kai Liu, ${ }^{\S}$ Jiang, Weng, ${ }^{*,+}$ Wei-Hua Huang, ${ }^{\dagger}$ Wei-Jie Huang, ${ }^{\ddagger}$ Qing Chen, ${ }^{\ddagger}$ Ning Lin, ${ }^{*, \neq}$ and Gui Lu${ }^{*,+}$

${ }^{\dagger}$ Guangdong Provincial Key Laboratory of Chiral Molecule and Drug Discovery, School of Pharmaceutical Sciences, Sun Yat-sen University, Guangzhou 510006, P. R. China.

‡ College of Pharmacy, Guangxi Zhuang Yao Medicine Center of Engineering and Technology, Guangxi University of Chinese Medicine, Nanning, Guangxi, 530200, P. R. China.

$\S$ Institute of Marine Drugs, Guangxi University of Chinese Medicine, Nanning, 530200, P. R. China.

E-mail for J.W.: wengj2@mail.sysu.edu.cn E-mail for N.L.: linning@gxtcmu.edu.cn

E-mail for G.L.: lugui@mail.sysu.edu.cn 


\section{Table of Contents}

1. General Information S3

2. Experimental Procedure and Characterization of Products S3

a. General Procedure to Prepare Racemic Products 3a-3v S3

b. General Procedure to Prepare Chiral Products 3a-3v S3

c. Analytical Data for Products 3a-3v S4

d. General Procedure to Prepare Racemic Products 8a-8m S11

e. General Procedure to Prepare Chiral Products 8a-8m S11

f. Analytical Data for Products 8a-8m $\quad \mathbf{S 1 2}$

g. Three Components Reaction $\mathbf{S 1 6}$

h. The Gram Scale Reaction of 3a and 8a $\quad$ S17

$\begin{array}{ll}\text { 3. Proposed Reaction Mechanism } & \mathbf{S 1 9}\end{array}$

4. Data for X-ray Crystal Structures of 3d and 8d S20

5. Copies of NMR Spectra of Compounds S22

6. Copies of HPLC Spectra of Compounds S76

\begin{tabular}{lr} 
7. & References \\
\hline
\end{tabular} 


\section{General Information}

All reactions were carried out in oven-dried reaction vessel unless otherwise noted and solvents were dried according to established procedures. Reactions were monitored by thin layer chromatography (TLC). Purification of reaction product was carried out by flash chromatography using Qing Dao Sea Chemical Reagent silica gel (200-300 mesh). ${ }^{1} \mathrm{H},{ }^{13} \mathrm{C}$ and ${ }^{19} \mathrm{~F}$ NMR spectra were recorded on Bruker $400 \mathrm{MHz}$ or 500 $\mathrm{MHz}$ spectrometer in $\mathrm{CDCl}_{3}$ unless otherwise noted. Chemical shifts in ${ }^{1} \mathrm{H}$ NMR spectra are reported in parts per million (ppm, $\delta$ ) downfield from the internal standard $\mathrm{Me}_{4} \mathrm{Si}(\mathrm{TMS}, \delta=0 \mathrm{ppm})$. Chemical shifts in ${ }^{13} \mathrm{C}$ NMR spectra are reported relative to the central line of the chloroform signal $(\delta=77.0 \mathrm{ppm})$. Data are presented as follows: chemical shift, integration, multiplicity ( $s=$ singlet, $d=$ doublet, $t=$ triplet, $q=$ quartet, $m=$ multiplet) and coupling constant in Hertz $(\mathrm{Hz})$. All ${ }^{19} \mathrm{~F}$ NMR spectra in this manuscript are proton-decoupled. HPLC analyses were conducted on an Agilent instrument using a Daicel Chiralpak IA, IB and IC column. High resolution mass spectra were obtained with a Shimadzu LCMS-IT-TOF mass spectrometer. The single crystal X-ray diffraction studies were carried out on a Xcalibur Onyx Nova diffractometer equipped with CuK/a radiation. The molecular sieves were previously activated through high temperature treatment. All low temperature reactions were carried out with EYELA PSL-1400 Low-temperature (Constant Temperature) Magnetic Stirring Reactor in EtOH bath.

Substrates $\mathbf{1},{ }^{1 \mathrm{a}} \mathbf{2},{ }^{1 \mathrm{c}}$ and $\mathbf{7}^{1 \mathrm{~b}}$ were known compounds and were synthesized according to literature method.

\section{Experimental Procedure and Characterization of Products}

\section{a. General Procedure to Prepare Racemic Products 3a-3v}

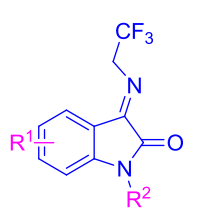

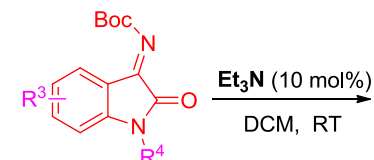

2

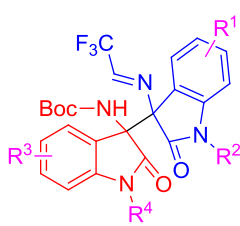

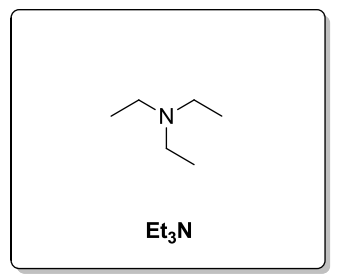

To a solution of $\mathrm{N}$-2,2,2-trifluoroethylisatin ketimine 1 ( $0.10 \mathrm{mmol}, 1.0$ equiv.), and catalyst $\mathrm{Et}_{3} \mathrm{~N}(0.01 \mathrm{mmol}$, $10 \mathrm{~mol} \%)$ in DCM (1 mL) was added isatin-derived $N$-Boc ketimine $2(0.10 \mathrm{mmol}, 1.0$ equiv.). The mixture was stirred at room temperature until the reaction was completed (monitored by TLC analysis). The crude product was purified directly by flash column chromatography on silica gel (petroleum ether/ ethyl acetate = 10:1 5:1) to give the desired racemic products $\mathbf{3 a - 3} \mathbf{v}$.

\section{b. General Procedure to Prepare Chiral Products 3a-3v}<smiles>[R]N1C(=O)/C(=N\CC(F)(F)F)c2cc[R1]cc21</smiles>

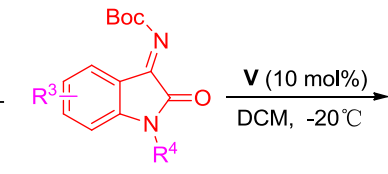

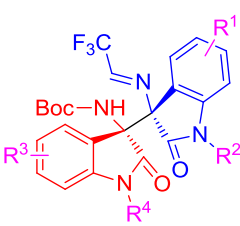

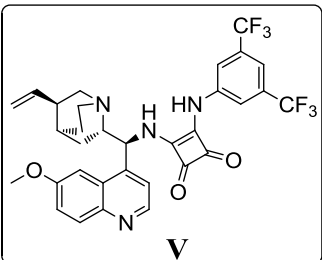

To a solution of $N$-2,2,2-trifluoroethylisatin ketimine 1 ( $0.10 \mathrm{mmol}, 1.0$ equiv.), $40 \mathrm{mg} 4 \AA$ molecular sieves, and catalyst $\mathbf{V}(0.01 \mathrm{mmol}, 10 \mathrm{~mol} \%)$ in DCM $(1 \mathrm{~mL})$ was added isatin-derived $N$-Boc ketimine $2(0.10 \mathrm{mmol}$, 1.0 equiv.). The mixture was stirred at $-20{ }^{\circ} \mathrm{C}$ until the reaction was completed (monitored by TLC analysis). 
The crude product was purified directly by flash column chromatography on silica gel (petroleum ether/ ethyl acetate $=10: 1 \sim 5: 1)$ to give the desired chiral products $\mathbf{3 a - 3} \mathbf{v}$.

\section{c. Analytical Data for Products 3a-3v}

tert-butyl ((3R,3'S)-1,1'-dimethyl-2,2'-dioxo-3'-((E)-(2,2,2-trifluoroethylidene)amino)-[3,3'-biindolin]-3-yl) carbamate $(\mathbf{3 a})$ : petroleum ether/ ethyl acetate $=5 / 1$

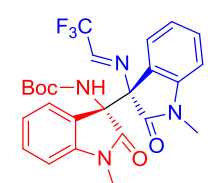

White solid, $46.7 \mathrm{mg}, 93 \%$ yield, $>20: 1 \mathrm{dr}$, 95\% ee, $[a]_{D}^{20}=+35.00(\mathrm{c}=0.26, \mathrm{MeOH})$.

${ }^{1} \mathrm{H}$ NMR $\left(400 \mathrm{MHz}, \mathrm{CDCl}_{3}\right) \delta: 7.47$ (q, $\left.J=2.9 \mathrm{~Hz}, 1 \mathrm{H}\right), 7.38(\mathrm{t}, J=7.7 \mathrm{~Hz}, 2 \mathrm{H}), 7.31-7.24(\mathrm{~m}, 1 \mathrm{H}), 7.19(\mathrm{~s}, 1 \mathrm{H})$, $7.11(\mathrm{t}, J=7.4 \mathrm{~Hz}, 1 \mathrm{H}), 6.84(\mathrm{~d}, J=7.9 \mathrm{~Hz}, 1 \mathrm{H}), 6.79-6.64(\mathrm{~m}, 2 \mathrm{H}), 5.67(\mathrm{~s}, 1 \mathrm{H}), 3.25(\mathrm{~s}, 3 \mathrm{H}), 2.73(\mathrm{~s}, 3 \mathrm{H}), 1.25$ (s, 9H); ${ }^{13} \mathrm{C}$ NMR $\left(101 \mathrm{MHz}, \mathrm{CDCl}_{3}\right) \delta: 172.9,170.9,154.2,152.5$ (q, $\left.J=39.2 \mathrm{~Hz}\right), 144.7,143.9,131.1,130.0$, 125.8, 125.6, 125.2, 122.6, 122.5, $122.1,118.9$ (q, $J=275.5 \mathrm{~Hz}$ ), 109.2, 108.0, 80.3, 72.2, 66.9, 28.2, 26.7, 26.0; ${ }^{19} \mathrm{~F}$ NMR (376 MHz, CDCl 3 ) $\delta:-70.85$. HRMS (ESI-TOF): $\mathrm{m} / z$ calcd. for $\mathrm{C}_{25} \mathrm{H}_{25} \mathrm{~N}_{4} \mathrm{NaO}_{4} \mathrm{~F}_{3}[\mathrm{M}+\mathrm{Na}]^{+}$: 525.1720; found: 525.1725. HPLC analysis: The enantiomeric excess was determined by HPLC with Chiralpak IC column (hexane $/ \mathrm{i}-\mathrm{PrOH}=80 / 20$, flow rate $0.8 \mathrm{~mL} \cdot \mathrm{min}^{-1}, \lambda=254 \mathrm{~nm}$ ): $t_{\text {major }}=10.6 \mathrm{~min}, t_{\text {minor }}=21.9$ $\min$.

tert-butyl ((3R,3'S)-5'-fluoro-1,1'-dimethyl-2,2'-dioxo-3'-((E)-(2,2,2-trifluoroethylidene)amino)-[3,3'-biindolin]-3-yl) carbamate $(\mathbf{3 b})$ : petroleum ether/ ethyl acetate $=5 / 1$

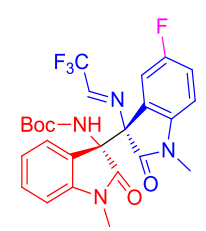

Yellow foam, $50.5 \mathrm{mg}, 97 \%$ yield, $>20: 1 \mathrm{dr}, 98 \%$ ee, $[a]_{D}^{20}=+28.00(\mathrm{c}=0.30, \mathrm{MeOH})$.

${ }^{1} \mathrm{H}$ NMR $\left(400 \mathrm{MHz}, \mathrm{CDCl}_{3}\right) \delta: 7.55(\mathrm{~d}, J=3.0 \mathrm{~Hz}, 1 \mathrm{H}), 7.41$ (t, $\left.J=7.7 \mathrm{~Hz}, 2 \mathrm{H}\right), 7.14$ (t, J=7.4 Hz, $\left.1 \mathrm{H}\right), 7.07$ (s, $1 \mathrm{H}), 7.00(\mathrm{td}, J=8.7,2.6 \mathrm{~Hz}, 1 \mathrm{H}), 6.87-6.66(\mathrm{~m}, 2 \mathrm{H}), 5.43(\mathrm{~s}, 1 \mathrm{H}) 3.24(\mathrm{~s}, 3 \mathrm{H}), 2.79(\mathrm{~s}, 3 \mathrm{H}), 1.24(\mathrm{~s}, 9 \mathrm{H}) ;{ }^{13} \mathrm{C}$ NMR $\left(101 \mathrm{MHz}, \mathrm{CDCl}_{3}\right) \delta: 172.7,170.8,158.5$ (d, $\left.J=242.8 \mathrm{~Hz}\right), 154.2,152.9$ (q, $\left.J=39.3 \mathrm{~Hz}\right), 144.6,139.8$, 130.3, 125.8, 125.4, 124.0 (d, $J=5.4 \mathrm{~Hz}$ ), 122.9, 118.8 (q, $J=275.8 \mathrm{~Hz}), 117.4(\mathrm{~d}, J=23.5 \mathrm{~Hz}), 113.4(\mathrm{~d}, J=$ $26.1 \mathrm{~Hz}$ ), 109.8 (d, $J=7.9 \mathrm{~Hz}), 108.1,80.5,72.1,66.9,28.2,26.8,26.2 ;{ }^{19} \mathrm{~F} \mathrm{NMR}\left(376 \mathrm{MHz}, \mathrm{CDCl}_{3}\right) \delta:-70.90$, -118.97. HRMS (ESI-TOF): $\mathrm{m} / \mathrm{z}$ calcd. for $\mathrm{C}_{25} \mathrm{H}_{24} \mathrm{~N}_{4} \mathrm{NaO}_{4} \mathrm{~F}_{4}[\mathrm{M}+\mathrm{Na}]^{+}: 543.1626$; found: 543.1620. HPLC analysis: The enantiomeric excess was determined by HPLC with Chiralpak IC column (hexane/ $i-\mathrm{PrOH}=$ $\left.80 / 20,0.8 \mathrm{~mL} \cdot \mathrm{min}^{-1}, \lambda=254 \mathrm{~nm}\right): t_{\text {major }}=9.3 \mathrm{~min}, t_{\text {minor }}=19.3 \mathrm{~min}$

tert-butyl ((3R,3'S)-5'-chloro-1,1'-dimethyl-2,2'-dioxo-3'-((E)-(2,2,2-trifluoroethylidene)amino)-[3,3'-biindolin]-3-yl) carbamate $(\mathbf{3 c})$ : petroleum ether/ ethyl acetate $=5 / 1$

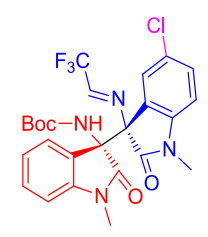


Yellow foam, $48.3 \mathrm{mg}, 90 \%$ yield, $>20: 1 \mathrm{dr}, 97 \%$ ee, $[a]_{D}^{20}=+1.45(\mathrm{c}=0.25, \mathrm{MeOH})$.

${ }^{1} \mathrm{H}$ NMR $\left(400 \mathrm{MHz}, \mathrm{CDCl}_{3}\right) \delta: 7.54$ (q, $\left.J=3.0 \mathrm{~Hz}, 1 \mathrm{H}\right), 7.43(\mathrm{td}, J=7.8,0.8 \mathrm{~Hz}, 1 \mathrm{H}), 7.36(\mathrm{~s}, 1 \mathrm{H}), 7.29-7.23(\mathrm{~m}$, $1 \mathrm{H}), 7.15(\mathrm{t}, J=7.5 \mathrm{~Hz}, 1 \mathrm{H}), 7.03(\mathrm{~s}, 1 \mathrm{H}), 6.76(\mathrm{dd}, J=8.1,5.1 \mathrm{~Hz}, 2 \mathrm{H}), 5.57(\mathrm{~s}, 1 \mathrm{H}), 3.24(\mathrm{~s}, 3 \mathrm{H}), 2.78(\mathrm{~s}, 3 \mathrm{H})$, 1.25 (s, 9H); ${ }^{13} \mathrm{C}$ NMR (101 MHz, $\left.\mathrm{CDCl}_{3}\right) \delta: 172.7,170.6,154.2,153.0$ (q, $J=39.4 \mathrm{~Hz}$ ), 144.5, 142.4, 130.9, 130.3, 130.3, 128.0, 125.7, 125.3, 123.9, 122.9, 118.8 (q, $J=275.8 \mathrm{~Hz}$ ), 110.1, 108.1, 80.6, 72.1, 67.0, 28.2, 26.8, 26.1; ${ }^{19} \mathrm{~F}$ NMR (376 MHz, CDCl $)$ ): -70.83 . HRMS (ESI-TOF): $m / z$ calcd. for $\mathrm{C}_{25} \mathrm{H}_{24} \mathrm{CIN}_{4} \mathrm{NaO}_{4} \mathrm{~F}_{3}[\mathrm{M}+\mathrm{Na}]^{+}$: 559.1330; found: 559.1333. HPLC analysis: The enantiomeric excess was determined by HPLC with Chiralpak IC column (hexane $/ \mathrm{i}-\mathrm{PrOH}=80 / 20,0.8 \mathrm{~mL} \cdot \mathrm{min}^{-1}, \lambda=254 \mathrm{~nm}$ ): $t_{\text {major }}=8.9 \mathrm{~min}, t_{\text {minor }}=17.8 \mathrm{~min}$.

tert-butyl ((3R,3'S)-5'-bromo-1,1'-dimethyl-2,2'-dioxo-3'-((E)-(2,2,2-trifluoroethylidene)amino)-[3,3'-biindolin]-3$\mathrm{yl})$ carbamate $(\mathbf{3 d})$ : petroleum ether/ ethyl acetate $=5 / 1$

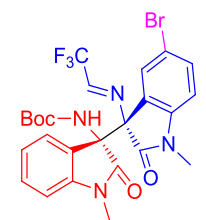

Yellow solid, $51.7 \mathrm{mg}, 89 \%$ yield, $>20: 1 \mathrm{dr}, 97 \%$ ee, $[a]_{D}^{20}=-5.77(\mathrm{c}=0.26, \mathrm{MeOH})$.

${ }^{1} \mathrm{H}$ NMR $\left(400 \mathrm{MHz}, \mathrm{CDCl}_{3}\right)$ ס: $7.54(\mathrm{q}, J=2.9 \mathrm{~Hz}, 1 \mathrm{H}), 7.49-7.28(\mathrm{~m}, 3 \mathrm{H}), 7.15(\mathrm{t}, J=7.4 \mathrm{~Hz}, 1 \mathrm{H}), 7.01(\mathrm{~s}, 1 \mathrm{H})$, $6.74(\mathrm{dd}, J=18.2,8.1 \mathrm{~Hz}, 2 \mathrm{H}), 5.68(\mathrm{~s}, 1 \mathrm{H}), 3.23(\mathrm{~s}, 3 \mathrm{H}), 2.77(\mathrm{~s}, 3 \mathrm{H}), 1.25(\mathrm{~s}, 9 \mathrm{H}) ;{ }^{13} \mathrm{C} \mathrm{NMR}\left(101 \mathrm{MHz}, \mathrm{CDCl}_{3}\right)$ $\delta: 172.6,170.5,154.2,153.0$ (q, $J=39.4 \mathrm{~Hz}$ ), 144.5, 142.9, 133.8, 130.3, 128.5, 125.7, 125.3, 124.2, 122.9 (d, $J=9.2 \mathrm{~Hz}$ ), $118.8(\mathrm{q}, J=275.7 \mathrm{~Hz}), 115.1,110.5,108.1,80.6,72.2,67.1,28.2,26.8,26.1 ;{ }^{19} \mathrm{~F} \mathrm{NMR}(376 \mathrm{MHz}$,

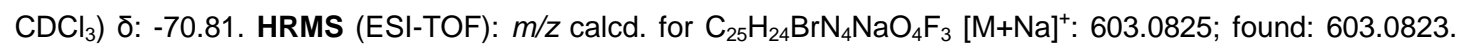
HPLC analysis: The enantiomeric excess was determined by HPLC with Chiralpak IC column (hexane/ $i-\mathrm{PrOH}$ $\left.=80 / 20,0.8 \mathrm{~mL} \cdot \mathrm{min}^{-1}, \lambda=254 \mathrm{~nm}\right): t_{\text {major }}=9.2 \mathrm{~min}, t_{\text {minor }}=18.1 \mathrm{~min}$.

tert-butyl $\quad\left(\left(3 R, 3^{\prime} S\right)-1,1^{\prime}, 5^{\prime}\right.$-trimethyl-2,2'-dioxo-3'-((E)-(2,2,2-trifluoroethylidene)amino)-[3,3'-biindolin]-3-yl) carbamate $(3 \mathbf{e})$ : petroleum ether/ ethyl acetate $=5 / 1$

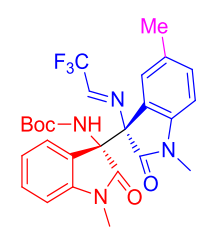

Yellow foam, $48.6 \mathrm{mg}, 94 \%$ yield, $>20: 1 \mathrm{dr}, 98 \%$ ee, $[a]_{D}^{20}=+13.14(\mathrm{c}=0.35, \mathrm{MeOH})$.

${ }^{1} \mathrm{H}$ NMR $\left(400 \mathrm{MHz}, \mathrm{CDCl}_{3}\right) \delta: 7.48$ (q, $\left.J=2.9 \mathrm{~Hz}, 1 \mathrm{H}\right), 7.39$ (t, $\left.J=7.6 \mathrm{~Hz}, 2 \mathrm{H}\right), 7.13$ (dd, $J=15.9,8.9 \mathrm{~Hz}, 2 \mathrm{H}$ ), $7.07(\mathrm{~d}, J=7.9 \mathrm{~Hz}, 1 \mathrm{H}), 6.78-6.62(\mathrm{~m}, 2 \mathrm{H}), 5.36(\mathrm{~s}, 1 \mathrm{H}), 3.22(\mathrm{~s}, 3 \mathrm{H}), 2.72(\mathrm{~s}, 3 \mathrm{H}), 2.02(\mathrm{~s}, 3 \mathrm{H}), 1.25(\mathrm{~s}, 9 \mathrm{H})$; ${ }^{13} \mathrm{C}$ NMR $\left(126 \mathrm{MHz}, \mathrm{CDCl}_{3}\right) \delta: 172.8,170.9,154.3,152.5$ (q, $\left.J=39.1 \mathrm{~Hz}\right), 144.8,141.5,132.0,131.1,129.9$, 126.0, 125.9, 125.6, 122.6, 122.2, 119.0 (q, $J=275.7 \mathrm{~Hz}$ ), 108.9, 107.7, 80.3, 72.4, 67.0, 28.2, 26.7, 26.0, 20.9; ${ }^{19}$ F NMR (376 MHz, CDCl ${ }_{3}$ ) : -70.79. HRMS (ESI-TOF): $\mathrm{m} / z$ calcd. for $\mathrm{C}_{26} \mathrm{H}_{27} \mathrm{~N}_{4} \mathrm{NaO}_{4} \mathrm{~F}_{3}[\mathrm{M}+\mathrm{Na}]^{+}: 539.1877$; found: 539.1873. HPLC analysis: The enantiomeric excess was determined by HPLC with Chiralpak IC column (hexane $/ \mathrm{i}-\mathrm{PrOH}=80 / 20,0.8 \mathrm{~mL} \cdot \mathrm{min}^{-1}, \lambda=254 \mathrm{~nm}$ ): $t_{\text {major }}=12.5 \mathrm{~min}, t_{\text {minor }}=25.6 \mathrm{~min}$.

tert-butyl ((3R,3'S)-5'-methoxy-1,1'-dimethyl-2,2'-dioxo-3'-((E)-(2,2,2-trifluoroethylidene)amino)-[3,3'-biindolin]3 -yl)carbamate (3f): petroleum ether/ ethyl acetate $=5 / 1$ 


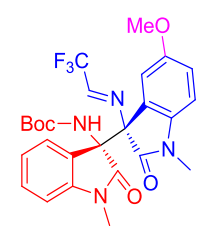

Yellow foam, $52.2 \mathrm{mg}, 98 \%$ yield, $>20: 1 \mathrm{dr},>99 \%$ ee, $[a]_{D}^{20}=+18.44(\mathrm{c}=0.32, \mathrm{MeOH})$.

${ }^{1} \mathrm{H}$ NMR $\left(400 \mathrm{MHz}, \mathrm{CDCl}_{3}\right) \delta: 7.52(\mathrm{q}, J=3.0 \mathrm{~Hz}, 1 \mathrm{H}), 7.39(\mathrm{td}, J=7.7,1.0 \mathrm{~Hz}, 2 \mathrm{H}), 7.13(\mathrm{t}, J=7.5 \mathrm{~Hz}, 2 \mathrm{H})$, $6.81(\mathrm{dd}, J=8.6,2.6 \mathrm{~Hz}, 1 \mathrm{H}), 6.77-6.58(\mathrm{~m}, 2 \mathrm{H}), 5.26(\mathrm{~s}, 1 \mathrm{H}), 3.43(\mathrm{~s}, 3 \mathrm{H}), 3.23(\mathrm{~s}, 3 \mathrm{H}), 2.77(\mathrm{~s}, 3 \mathrm{H}), 1.24(\mathrm{~s}$, 9H); ${ }^{13} \mathrm{C}$ NMR $\left(101 \mathrm{MHz}, \mathrm{CDCl}_{3}\right) \delta: 172.9,170.8,155.5,154.3,152.7$ (q, J = 39.2 Hz), 144.8, 137.1, 130.0, 129.9, 125.9, 123.4, 122.7, 118.9 (q, $J=275.8 \mathrm{~Hz}$ ), 116.7, 111.1, 109.8, 107.9, 80.4, 72.2, 66.9, 55.6, 28.2, 26.7, 26.1; ${ }^{19} \mathrm{~F}$ NMR (376 MHz, CDCl 3 ) $\delta:-70.84$. HRMS (ESI-TOF): $\mathrm{m} / z$ calcd. for $\mathrm{C}_{26} \mathrm{H}_{27} \mathrm{~N}_{4} \mathrm{NaO}_{5} \mathrm{~F}_{3}[\mathrm{M}+\mathrm{Na}]^{+}$: 555.1826; found: 555.1827. HPLC analysis: The enantiomeric excess was determined by HPLC with Chiralpak IC column (hexane $/ \mathrm{i}-\mathrm{PrOH}=80 / 20,0.8 \mathrm{~mL} \cdot \mathrm{min}^{-1}, \lambda=254 \mathrm{~nm}$ ): $t_{\text {major }}=13.2 \mathrm{~min}, t_{\text {minor }}=28.7 \mathrm{~min}$.

tert-butyl ((3R,3'S)-6'-chloro-1,1'-dimethyl-2,2'-dioxo-3'-((E)-(2,2,2-trifluoroethylidene)amino)-[3,3'-biindolin]-3-yl) carbamate $(\mathbf{3 g})$ : petroleum ether/ ethyl acetate $=5 / 1$

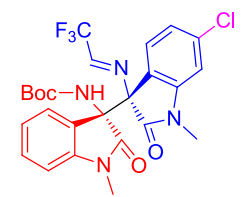

Yellow foam, $46.7 \mathrm{mg}, 87 \%$ yield, $>20: 1 \mathrm{dr}, 93 \%$ ee, $[a]_{D}^{20}=+55.79(\mathrm{c}=0.19$, MeOH).

${ }^{1} \mathrm{H}$ NMR $\left(400 \mathrm{MHz}, \mathrm{CDCl}_{3}\right) \delta: 7.48$ (q, $\left.J=2.7 \mathrm{~Hz}, 1 \mathrm{H}\right), 7.39$ (t, $\left.J=7.7 \mathrm{~Hz}, 2 \mathrm{H}\right), 7.12(\mathrm{t}, J=7.4 \mathrm{~Hz}, 1 \mathrm{H}), 7.06(\mathrm{~s}$, $1 \mathrm{H}), 6.84(\mathrm{~d}, J=1.7 \mathrm{~Hz}, 1 \mathrm{H}), 6.71(\mathrm{t}, J=6.1 \mathrm{~Hz}, 2 \mathrm{H}), 5.58(\mathrm{~s}, 1 \mathrm{H}), 3.24(\mathrm{~s}, 3 \mathrm{H}), 2.80(\mathrm{~s}, 3 \mathrm{H}), 1.24(\mathrm{~s}, 9 \mathrm{H}) ;{ }^{13} \mathrm{C}$ NMR $\left(101 \mathrm{MHz}, \mathrm{CDCl}_{3}\right) \delta: 172.8,171.0,154.1,152.8$ (q, $\left.J=39.3 \mathrm{~Hz}\right), 145.1,144.6,137.2,130.2,126.2,125.7$, 125.6, 122.8, 122.4, 120.6, 118.8 (q, $J=275.9 \mathrm{~Hz}$ ), 110.0, 108.2, 80.6, 71.8, 66.9, 28.2, 26.8, 26.2; ${ }^{19} \mathrm{~F}$ NMR (376 MHz, $\mathrm{CDCl}_{3}$ ) $\delta:-70.85$. HRMS (ESI-TOF): $\mathrm{m} / z$ calcd. for $\mathrm{C}_{25} \mathrm{H}_{24} \mathrm{CIN}_{4} \mathrm{NaO}_{4} \mathrm{~F}_{3}[\mathrm{M}+\mathrm{Na}]^{+}:$559.1330; found: 559.1331. HPLC analysis: The enantiomeric excess was determined by HPLC with Chiralpak IC column (hexane $/ \mathrm{i}-\mathrm{PrOH}=80 / 20,0.8 \mathrm{~mL} \cdot \mathrm{min}^{-1}, \lambda=254 \mathrm{~nm}$ ): $t_{\text {major }}=9.3 \mathrm{~min}, t_{\text {minor }}=18.2 \mathrm{~min}$.

tert-butyl ((3R,3'S)-7'-chloro-1,1'-dimethyl-2,2'-dioxo-3'-((E)-(2,2,2-trifluoroethylidene)amino)-[3,3'-biindolin]-3-yl) carbamate $(\mathbf{3 h})$ : petroleum ether/ ethyl acetate $=10 / 1$

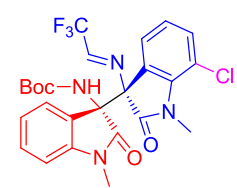

Yellow foam, $48.9 \mathrm{mg}, 91 \%$ yield, $>20: 1 \mathrm{dr}, 95 \%$ ee, $[a]_{D}^{20}=+51.00(\mathrm{c}=0.20, \mathrm{MeOH})$.

${ }^{1} \mathrm{H}$ NMR $\left(400 \mathrm{MHz}, \mathrm{CDCl}_{3}\right) \delta: 7.52(\mathrm{~d}, J=3.0 \mathrm{~Hz}, 1 \mathrm{H}), 7.39(\mathrm{td}, J=7.7,0.9 \mathrm{~Hz}, 2 \mathrm{H}), 7.20$ (dd, $J=8.2,0.9 \mathrm{~Hz}$, $1 \mathrm{H}), 7.12(\mathrm{t}, J=7.5 \mathrm{~Hz}, 1 \mathrm{H}), 7.07(\mathrm{~s}, 1 \mathrm{H}), 6.69(\mathrm{~d}, J=7.7 \mathrm{~Hz}, 1 \mathrm{H}), 6.63(\mathrm{t}, J=7.7 \mathrm{~Hz}, 1 \mathrm{H}), 5.60(\mathrm{~s}, 1 \mathrm{H}), 3.62(\mathrm{~s}$, $3 \mathrm{H}), 2.80(\mathrm{~s}, 3 \mathrm{H}), 1.24(\mathrm{~s}, 9 \mathrm{H}) ;{ }^{13} \mathrm{C}$ NMR $\left(101 \mathrm{MHz}, \mathrm{CDCl}_{3}\right) \delta: 172.8,171.5,154.2,152.9$ (q, J = 39.4 Hz), 144.7 , 139.8, 133.4, 130.2, 125.8, 125.5, 125.3, 123.8, 123.2, 122.8, 118.8 (q, $J=283.0 \mathrm{~Hz}$ ), 116.5, 108.1, 80.5, 71.4, 67.0, 30.2, 28.2, 26.2; ${ }^{19} \mathrm{~F}$ NMR (376 MHz, $\mathrm{CDCl}_{3}$ ) $\delta:-70.90$. HRMS (ESI-TOF): $\mathrm{m} / \mathrm{z}$ calcd. for $\mathrm{C}_{25} \mathrm{H}_{24} \mathrm{CIN}_{4} \mathrm{NaO}_{4} \mathrm{~F}_{3}[\mathrm{M}+\mathrm{Na}]^{+}:$559.1330; found: 559.1330. HPLC analysis: The enantiomeric excess was determined by HPLC with Chiralpak IC column (hexane $/ \mathrm{i}-\mathrm{PrOH}=80 / 20,0.8 \mathrm{~mL} \cdot \mathrm{min}^{-1}, \lambda=254 \mathrm{~nm}$ ): $t_{\text {major }}=8.1$ $\min , t_{\text {minor }}=13.7 \mathrm{~min}$. 
tert-butyl ((3R,3'S)-1-methyl-2,2'-dioxo-1'-phenyl-3'-((E)-(2,2,2-trifluoroethylidene)amino)-[3,3'-biindolin]-3-yl) carbamate (3i): petroleum ether/ ethyl acetate $=10 / 1$

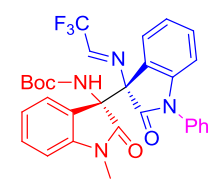

Yellow foam, $53.6 \mathrm{mg}, 95 \%$ yield, $>20: 1 \mathrm{dr}, 92 \%$ ee, $[a]_{D}^{20}=+53.56(\mathrm{c}=0.45, \mathrm{MeOH})$.

${ }^{1} \mathrm{H}$ NMR $\left(400 \mathrm{MHz}, \mathrm{CDCl}_{3}\right) \delta: 7.66(\mathrm{~s}, 1 \mathrm{H}), 7.63-7.28(\mathrm{~m}, 7 \mathrm{H}), 7.21(\mathrm{t}, J=7.7 \mathrm{~Hz}, 1 \mathrm{H}), 7.14(\mathrm{~s}, 2 \mathrm{H}), 6.73(\mathrm{t}, J=$ $6.4 \mathrm{~Hz}, 3 \mathrm{H}), 5.68(\mathrm{~s}, 1 \mathrm{H}), 2.78(\mathrm{~s}, 3 \mathrm{H}), 1.24(\mathrm{~s}, 9 \mathrm{H}) ;{ }^{13} \mathrm{C} \mathrm{NMR}\left(101 \mathrm{MHz}, \mathrm{CDCl}_{3}\right) \delta: 172.9,170.5,154.2,152.8$ (q, $J=39.3 \mathrm{~Hz}$ ), 144.8, 144.2, 144.2, 133.3, 131.0, 130.1, 129.9, 129.0, 127.2, 125.6, 125.5, 122.8, 122.6, 118.9 (q, $J=275.8 \mathrm{~Hz}), 110.3,108.1,80.4,72.1,67.3,28.1,26.0 ;{ }^{19} \mathrm{~F} \mathrm{NMR}\left(376 \mathrm{MHz}, \mathrm{CDCl}_{3}\right) \delta:-70.72$. HRMS (ESI-TOF): $\mathrm{m} / z$ calcd. for $\mathrm{C}_{30} \mathrm{H}_{27} \mathrm{~N}_{4} \mathrm{NaO}_{4} \mathrm{~F}_{3}[\mathrm{M}+\mathrm{Na}]^{+}:$587.1877; found: 587.1878. HPLC analysis: The enantiomeric excess was determined by HPLC with Chiralpak IC column (hexane/i-PrOH =90/10, 0.8 $\mathrm{mL} \cdot \mathrm{min}^{-1}$, $\lambda=254 \mathrm{~nm}): t_{\text {major }}=12.6 \mathrm{~min}, t_{\text {minor }}=34.3 \mathrm{~min}$.

tert-butyl ((3R,3'S)-1'-benzyl-1-methyl-2,2'-dioxo-3'-((E)-(2,2,2-trifluoroethylidene)amino)-[3,3'-biindolin]-3-yl) carbamate $(\mathbf{3 j})$ : petroleum ether/ ethyl acetate $=10 / 1$

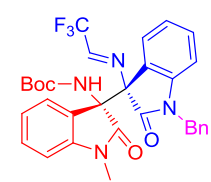

Yellow foam, $53.8 \mathrm{mg}, 93 \%$ yield, >20:1 dr, 92\% ee, $[a]_{D}^{20}=+22.44(\mathrm{c}=0.45, \mathrm{MeOH})$.

${ }^{1} \mathrm{H}$ NMR $\left(400 \mathrm{MHz}, \mathrm{CDCl}_{3}\right) \delta: 7.44(\mathrm{~s}, 1 \mathrm{H}), 7.39(\mathrm{t}, J=7.9 \mathrm{~Hz}, 1 \mathrm{H}), 7.35-7.23(\mathrm{~m}, 6 \mathrm{H}), 7.20-7.05(\mathrm{~m}, 3 \mathrm{H}), 6.72$ $(\mathrm{d}, J=7.8 \mathrm{~Hz}, 2 \mathrm{H}), 6.66(\mathrm{~d}, J=7.9 \mathrm{~Hz}, 1 \mathrm{H}), 5.64(\mathrm{~s}, 1 \mathrm{H}), 4.99(\mathrm{~d}, J=14.7 \mathrm{~Hz}, 1 \mathrm{H}), 4.90(\mathrm{~d}, J=15.5 \mathrm{~Hz}, 1 \mathrm{H})$, $2.81(\mathrm{~s}, 3 \mathrm{H}), 1.26(\mathrm{~s}, 9 \mathrm{H}) ;{ }^{13} \mathrm{C}$ NMR (126 MHz, $\mathrm{CDCl}_{3}$ ) $\delta: 172.9,154.2,152.4$ (q, $\left.J=39.2 \mathrm{~Hz}\right), 144.8,143.1$, 135.0, 134.7, 131.0, 130.1, 129.0, 127.9, 127.4, 125.8, 125.6, 125.5, 122.6, 122.5, 122.5, 118.9 (q, $J=275.7$ $\mathrm{Hz}), 110.4,108.1,80.4,72.4,66.6,44.6,28.2,26.1 ;{ }^{19} \mathrm{~F}$ NMR (376 MHz, $\left.\mathrm{CDCl}_{3}\right)$ ס: -70.81. HRMS (ESI-TOF): $\mathrm{m} / \mathrm{z}$ calcd. for $\mathrm{C}_{31} \mathrm{H}_{29} \mathrm{~N}_{4} \mathrm{NaO}_{4} \mathrm{~F}_{3}[\mathrm{M}+\mathrm{Na}]^{+}:$601.2033; found: 601.2030. HPLC analysis: The enantiomeric excess was determined by HPLC with Chiralpak IC column (hexane $/ \mathrm{i}-\mathrm{PrOH}=90 / 10,0.8 \mathrm{~mL} \cdot \mathrm{min}^{-1}, \lambda=254 \mathrm{~nm}$ ): $t_{\text {major }}=17.2 \mathrm{~min}, t_{\text {minor }}=26.7 \mathrm{~min}$.

tert-butyl ((3R,3'S)-5-fluoro-1,1'-dimethyl-2,2'-dioxo-3'-((E)-(2,2,2-trifluoroethylidene)amino)-[3,3'-biindolin]-3-yl) carbamate $(\mathbf{3 k})$ : petroleum ether/ ethyl acetate $=5 / 1$

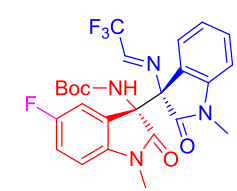

Yellow oil, $47.9 \mathrm{mg}, 92 \%$ yield, $>20: 1 \mathrm{dr}, 94 \%$ ee, $[a]_{D}^{20}=+16.13(\mathrm{c}=0.31, \mathrm{MeOH})$.

${ }^{1} \mathrm{H}$ NMR $\left(400 \mathrm{MHz}, \mathrm{CDCl}_{3}\right) \delta: 7.52(\mathrm{dd}, J=5.9,2.9 \mathrm{~Hz}, 1 \mathrm{H}), 7.29(\mathrm{t}, J=7.8 \mathrm{~Hz}, 1 \mathrm{H}), 7.23-6.87(\mathrm{~m}, 3 \mathrm{H}), 6.85(\mathrm{~d}$, $J=7.9 \mathrm{~Hz}, 1 \mathrm{H}), 6.75(\mathrm{t}, J=7.5 \mathrm{~Hz}, 1 \mathrm{H}), 6.62(\mathrm{dd}, J=8.5,4.0 \mathrm{~Hz}, 1 \mathrm{H}), 5.78(\mathrm{~s}, 1 \mathrm{H}), 3.25(\mathrm{~s}, 3 \mathrm{H}), 2.72(\mathrm{~s}, 3 \mathrm{H})$, 1.27 (s, 9H); ${ }^{13} \mathrm{C}$ NMR (126 MHz, $\left.\mathrm{CDCl}_{3}\right) \delta: 172.7,170.9,159.1$ (d, $\left.J=241.5 \mathrm{~Hz}\right), 154.3,152.9$ (q, $J=39.2 \mathrm{~Hz}$ ), 143.7, 140.8, 131.2, 125.0, 122.6, 122.4, 118.9 (q, $J=275.7 \mathrm{~Hz}$ ), 116.4, 116.2, 114.1 (d, $J=25.3 \mathrm{~Hz}$ ), 109.4, $108.4(\mathrm{~d}, J=7.8 \mathrm{~Hz}), 80.7,71.9,67.1,28.2,26.7,26.2 ;{ }^{19} \mathrm{~F} \mathrm{NMR}\left(376 \mathrm{MHz}, \mathrm{CDCl}_{3}\right) \delta:-70.90,-119.74$. HRMS (ESI-TOF): $\mathrm{m} / \mathrm{z}$ calcd. for $\mathrm{C}_{25} \mathrm{H}_{24} \mathrm{~N}_{4} \mathrm{NaO}_{4} \mathrm{~F}_{4}[\mathrm{M}+\mathrm{Na}]^{+}:$543.1626; found: 543.1628. HPLC analysis: The enantiomeric excess was determined by HPLC with Chiralpak IC column (hexane/i-PrOH = 80/20, $0.8 \mathrm{~mL} \cdot \mathrm{min}^{-1}$, 
$\lambda=254 \mathrm{~nm}): t_{\text {major }}=7.4 \mathrm{~min}, t_{\text {minor }}=13.4 \mathrm{~min}$.

tert-butyl ((3R,3'S)-5-chloro-1,1'-dimethyl-2,2'-dioxo-3'-((E)-(2,2,2-trifluoroethylidene)amino)-[3,3'-biindolin]-3-yl) carbamate (3I): petroleum ether/ ethyl acetate $=5 / 1$

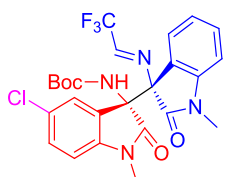

Yellow oil, $51.5 \mathrm{mg}, 96 \%$ yield, $>20: 1 \mathrm{dr}, 94 \%$ ee, $[a]_{D}^{20}=+45.12(\mathrm{c}=0.41, \mathrm{MeOH})$.

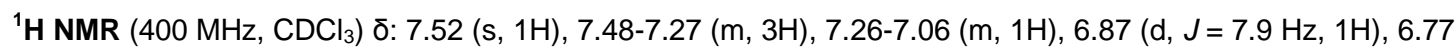
(t, $J=7.6 \mathrm{~Hz}, 1 \mathrm{H}), 6.64(\mathrm{~d}, J=7.2 \mathrm{~Hz}, 1 \mathrm{H}), 5.81(\mathrm{~s}, 1 \mathrm{H}), 3.26(\mathrm{~s}, 3 \mathrm{H}), 2.73(\mathrm{~s}, 3 \mathrm{H}), 1.29(\mathrm{~s}, 9 \mathrm{H}) ;{ }^{13} \mathrm{C}$ NMR $(126$ $\left.\mathrm{MHz}, \mathrm{CDCl}_{3}\right) \delta: 172.5,170.7,154.2,152.8$ (q, $\left.J=39.2 \mathrm{~Hz}\right), 143.7,143.2,131.2,129.8,128.0,127.3,126.1$, 124.9, 122.5, 122.0, 118.7 (q, $J=275.7 \mathrm{~Hz}), 109.3,108.8,80.6,71.8,66.8,28.1,26.6,26.0 ;{ }^{19} \mathrm{~F}$ NMR $(376$ $\mathrm{MHz}, \mathrm{CDCl}_{3}$ ) $\delta:-70.90$. HRMS (ESI-TOF): $\mathrm{m} / \mathrm{z}$ calcd. for $\mathrm{C}_{25} \mathrm{H}_{24} \mathrm{CIN}_{4} \mathrm{NaO}_{4} \mathrm{~F}_{3}[\mathrm{M}+\mathrm{Na}]^{+}:$559.1330; found: 559.1336. HPLC analysis: The enantiomeric excess was determined by HPLC with Chiralpak IC column (hexane $/ \mathrm{i}-\mathrm{PrOH}=80 / 20,0.8 \mathrm{~mL} \cdot \mathrm{min}^{-1}, \lambda=254 \mathrm{~nm}$ ): $t_{\text {major }}=7.1 \mathrm{~min}, t_{\text {minor }}=12.0 \mathrm{~min}$.

tert-butyl ((3R,3'S)-5-bromo-1,1'-dimethyl-2,2'-dioxo-3'-((E)-(2,2,2-trifluoroethylidene)amino)-[3,3'-biindolin]-3-yl) carbamate $(3 \mathrm{~m})$ : petroleum ether/ ethyl acetate $=5 / 1$

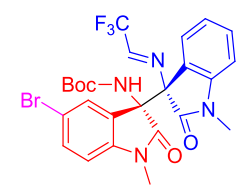

Yellow foam, $50.0 \mathrm{mg}, 86 \%$ yield, $>20: 1 \mathrm{dr}, 95 \%$ ee, $[a]_{D}^{20}=+47.14(\mathrm{c}=0.35, \mathrm{MeOH})$.

${ }^{1} \mathrm{H}$ NMR $\left(400 \mathrm{MHz}, \mathrm{CDCl}_{3}\right)$ ס: 7.69-7.37 (m, 3H), $7.32(\mathrm{t}, J=7.8 \mathrm{~Hz}, 1 \mathrm{H}), 7.19(\mathrm{~s}, 1 \mathrm{H}), 6.86(\mathrm{~d}, J=7.9 \mathrm{~Hz}, 1 \mathrm{H})$, $6.78(\mathrm{t}, J=7.4 \mathrm{~Hz}, 1 \mathrm{H}), 6.59(\mathrm{~d}, J=8.4 \mathrm{~Hz}, 1 \mathrm{H}), 5.81(\mathrm{~s}, 1 \mathrm{H}), 3.26(\mathrm{~s}, 3 \mathrm{H}), 2.72(\mathrm{~s}, 3 \mathrm{H}), 1.29(\mathrm{~s}, 9 \mathrm{H}) ;{ }^{13} \mathrm{C}$ NMR $\left(126 \mathrm{MHz}, \mathrm{CDCl}_{3}\right) \delta: 172.5,170.7,154.3,152.9$ (q, $\left.J=39.4 \mathrm{~Hz}\right), 143.8,143.8,132.8,132.4,131.3,131.0$, 127.8, 125.1, 122.6, 122.1, 118.9 (q, $J=275.9 \mathrm{~Hz}$ ), 115.3, 109.4, 80.7, 71.9, 66.8, 28.2, 26.7, 26.1; ${ }^{19} \mathrm{~F}$ NMR (376 MHz, $\mathrm{CDCl}_{3}$ ) $\delta:-70.89$. HRMS (ESI-TOF): $\mathrm{m} / z$ calcd. for $\mathrm{C}_{25} \mathrm{H}_{24} \mathrm{BrN}_{4} \mathrm{NaO}_{4} \mathrm{~F}_{3}[\mathrm{M}+\mathrm{Na}]^{+}: 603.0825$; found: 603.0834. HPLC analysis: The enantiomeric excess was determined by HPLC with Chiralpak IC column (hexane $/ \mathrm{i}-\mathrm{PrOH}=80 / 20,0.8 \mathrm{~mL} \cdot \mathrm{min}^{-1}, \lambda=254 \mathrm{~nm}$ ): $t_{\text {major }}=7.5 \mathrm{~min}, t_{\text {minor }}=13.0 \mathrm{~min}$.

tert-butyl ((3R,3'S)-1,1',5-trimethyl-2,2'-dioxo-3'-((E)-(2,2,2-trifluoroethylidene)amino)-[3,3'-biindolin]-3-yl) carbamate $(3 n)$ : petroleum ether/ ethyl acetate $=5 / 1$

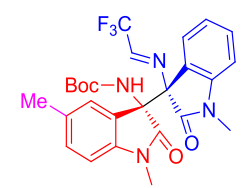

Yellow oil, $47.5 \mathrm{mg}, 92 \%$ yield, $>20: 1 \mathrm{dr}, 98 \%$ ee, $[a]_{D}^{20}=+63.24(\mathrm{c}=0.37, \mathrm{MeOH})$.

${ }^{1} \mathrm{H}$ NMR $\left(400 \mathrm{MHz}, \mathrm{CDCl}_{3}\right) \delta: 7.42(\mathrm{q}, J=3.0 \mathrm{~Hz}, 1 \mathrm{H}), 7.35-7.21(\mathrm{~m}, 2 \mathrm{H}), 7.17(\mathrm{~d}, J=7.8 \mathrm{~Hz}, 2 \mathrm{H}), 6.84(\mathrm{~d}, J=$ $7.8 \mathrm{~Hz}, 1 \mathrm{H}$ ), 6.72 (t, J=7.5 Hz, 1H), 6.57 (d, $J=7.9 \mathrm{~Hz}, 1 \mathrm{H}), 5.63(\mathrm{~s}, 1 \mathrm{H}), 3.25$ (s, 3H), 2.69 (s, 3H), 2.35 (s, $3 \mathrm{H}), 1.25(\mathrm{~s}, 9 \mathrm{H}) ;{ }^{13} \mathrm{C}$ NMR $\left(101 \mathrm{MHz}, \mathrm{CDCl}_{3}\right) \delta: 172.8,170.9,154.2,152.4(\mathrm{q}, J=39.2 \mathrm{~Hz}), 144.0,142.3$, 132.2, 131.1, 130.3, 126.4, 125.7, 125.4, 122.3, 121.8, 119.0 (q, $J=275.8 \mathrm{~Hz}$ ), 109.2, 107.7, 80.3, 72.3, 67.0, 28.2, 26.7, 26.0, 21.3; ${ }^{19} \mathrm{~F}$ NMR (376 MHz, $\mathrm{CDCl}_{3}$ ) ס: -70.85. HRMS (ESI-TOF): $\mathrm{m} / z$ calcd. for $\mathrm{C}_{26} \mathrm{H}_{27} \mathrm{~N}_{4} \mathrm{NaO}_{4} \mathrm{~F}_{3}$ $[\mathrm{M}+\mathrm{Na}]^{+}: 539.1877$; found: 539.1888. HPLC analysis: The enantiomeric excess was determined by HPLC with 
Chiralpak IC column (hexane $/ \mathrm{i}-\mathrm{PrOH}=80 / 20,0.8 \mathrm{~mL} \cdot \mathrm{min}^{-1}, \lambda=254 \mathrm{~nm}$ ): $t_{\text {major }}=10.8 \mathrm{~min}, t_{\text {minor }}=23.3 \mathrm{~min}$.

tert-butyl ((3R,3'S)-5-methoxy-1,1'-dimethyl-2,2'-dioxo-3'-((E)-(2,2,2-trifluoroethylidene)amino)-[3,3'-biindolin]3-yl)carbamate (30): petroleum ether/ ethyl acetate $=5 / 1$

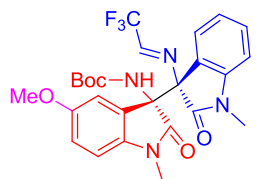

Yellow oil, $47.9 \mathrm{mg}, 90 \%$ yield, $>20: 1 \mathrm{dr}, 97 \%$ ee, $[a]_{D}^{20}=+59.00(\mathrm{c}=0.40, \mathrm{MeOH})$.

${ }^{1} \mathrm{H}$ NMR $\left(400 \mathrm{MHz}, \mathrm{CDCl}_{3}\right)$ ס: $7.47(\mathrm{~d}, J=2.9 \mathrm{~Hz}, 1 \mathrm{H}), 7.33-7.25(\mathrm{~m}, 1 \mathrm{H}), 7.21(\mathrm{~s}, 1 \mathrm{H}), 7.02(\mathrm{~s}, 1 \mathrm{H}), 6.92(\mathrm{dd}, J$ $=8.5,2.5 \mathrm{~Hz}, 1 \mathrm{H}), 6.84(\mathrm{~d}, J=7.8 \mathrm{~Hz}, 1 \mathrm{H}), 6.73(\mathrm{t}, J=7.4 \mathrm{~Hz}, 1 \mathrm{H}), 6.60(\mathrm{~d}, J=8.5 \mathrm{~Hz}, 1 \mathrm{H}), 5.67(\mathrm{~s}, 1 \mathrm{H}), 3.79$ (s, 3H), 3.25 (s, 3H), 2.69 (s, 3H), 1.24 (s, 9H); ${ }^{13} \mathrm{C}$ NMR (126 MHz, $\left.\mathrm{CDCl}_{3}\right)$ ס: 172.6, 170.9, 155.9, 154.2, 152.5 (q, $J=39.1 \mathrm{~Hz}$ ), 143.9, 138.2, 131.1, 126.8, 125.3, 122.4, 122.1, 119.0 (q, $J=266.9 \mathrm{~Hz}$ ), 115.7, 111.7, 109.2, 108.5, 80.4, 72.1, 67.3, 55.7, 28.2, 26.7, 26.1; ${ }^{19} \mathrm{~F}$ NMR (376 MHz, $\mathrm{CDCl}_{3}$ ) $\delta:-70.72$. HRMS (ESI-TOF): $\mathrm{m} / \mathrm{z}$ calcd. for $\mathrm{C}_{26} \mathrm{H}_{27} \mathrm{~N}_{4} \mathrm{NaO}_{5} \mathrm{~F}_{3}[\mathrm{M}+\mathrm{Na}]^{+}:$555.1826; found: 555.1834. HPLC analysis: The enantiomeric excess was determined by HPLC with Chiralpak IC column (hexane $/ \mathrm{i}-\mathrm{PrOH}=80 / 20,0.8 \mathrm{~mL} \cdot \mathrm{min}^{-1}, \lambda=254 \mathrm{~nm}$ ): $t_{\text {major }}=$ $12.1 \mathrm{~min}, t_{\text {minor }}=27.4 \mathrm{~min}$.

tert-butyl ((3R,3'S)-6-chloro-1,1'-dimethyl-2,2'-dioxo-3'-((E)-(2,2,2-trifluoroethylidene)amino)-[3,3'-biindolin]-3-yl) carbamate $(3 p)$ : petroleum ether/ ethyl acetate $=5 / 1$

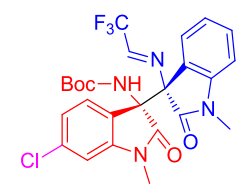

Yellow oil, $47.2 \mathrm{mg}, 88 \%$ yield, $>20: 1 \mathrm{dr}, 96 \%$ ee, $[a]_{D}^{20}=+43.50(\mathrm{c}=0.40, \mathrm{MeOH})$.

${ }^{1} \mathrm{H}$ NMR $\left(400 \mathrm{MHz}, \mathrm{CDCl}_{3}\right) \delta: 7.50$ (dd, $\left.J=5.9,2.8 \mathrm{~Hz}, 1 \mathrm{H}\right), 7.31$ (td, $\left.J=7.8,1.1 \mathrm{~Hz}, 2 \mathrm{H}\right), 7.18(\mathrm{~s}, 1 \mathrm{H}), 7.10$ (d, $J=7.4 \mathrm{~Hz}, 1 \mathrm{H}), 6.85(\mathrm{~d}, J=7.8 \mathrm{~Hz}, 1 \mathrm{H}), 6.80(\mathrm{t}, J=7.7 \mathrm{~Hz}, 1 \mathrm{H}), 6.70(\mathrm{~d}, J=1.8 \mathrm{~Hz}, 1 \mathrm{H}), 5.82(\mathrm{~s}, 1 \mathrm{H}), 3.25(\mathrm{~s}$, $3 \mathrm{H}), 2.72(\mathrm{~s}, 3 \mathrm{H}), 1.27$ (s, 9H); ${ }^{13} \mathrm{C}$ NMR (126 MHz, $\mathrm{CDCl}_{3}$ ) $\delta: 173.0,170.9,154.3,152.8$ (q, J=39.2 Hz), 145.9, 143.8, 135.9, 131.3, 126.6, 125.0, 124.2, 122.7, 122.6, 122.2, 118.9 (q, J = 275.7 Hz), 109.4, 108.8, 80.7, 71.9, 66.6, 28.2, 26.7, 26.2; ${ }^{19} \mathrm{~F}$ NMR (376 MHz, $\mathrm{CDCl}_{3}$ ) $\delta:-70.88$. HRMS (ESI-TOF): $\mathrm{m} / \mathrm{z}$ calcd. for $\mathrm{C}_{25} \mathrm{H}_{24} \mathrm{ClN}_{4} \mathrm{NaO}_{4} \mathrm{~F}_{3}[\mathrm{M}+\mathrm{Na}]^{+}:$559.1330; found: 559.1341. HPLC analysis: The enantiomeric excess was determined by HPLC with Chiralpak IC column (hexane $/ \mathrm{i}-\mathrm{PrOH}=80 / 20,0.8 \mathrm{~mL} \cdot \mathrm{min}^{-1}, \lambda=254 \mathrm{~nm}$ ): $t_{\text {major }}=7.4$ $\min , t_{\text {minor }}=12.2 \mathrm{~min}$.

tert-butyl ((3R,3'S)-6-methoxy-1,1'-dimethyl-2,2'-dioxo-3'-((E)-(2,2,2-trifluoroethylidene)amino)-[3,3'-biindolin]3-yl)carbamate (3q): petroleum ether/ ethyl acetate $=10 / 1$

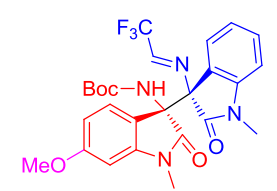

Yellow oil, $52.2 \mathrm{mg}, 98 \%$ yield, $>20: 1 d r,>99 \%$ ee, $[a]_{D}^{20}=+58.18(\mathrm{c}=0.11, \mathrm{MeOH})$.

${ }^{1} \mathrm{H}$ NMR $\left(400 \mathrm{MHz}, \mathrm{CDCl}_{3}\right) \delta: 7.47(\mathrm{~d}, J=3.1 \mathrm{~Hz}, 1 \mathrm{H}), 7.29(\mathrm{td}, J=7.9,1.0 \mathrm{~Hz}, 2 \mathrm{H}), 7.18(\mathrm{~s}, 1 \mathrm{H}), 6.84(\mathrm{~d}, J=$ $7.8 \mathrm{~Hz}, 1 \mathrm{H}), 6.76(\mathrm{t}, J=7.5 \mathrm{~Hz}, 1 \mathrm{H}), 6.60(\mathrm{~d}, J=7.5 \mathrm{~Hz}, 1 \mathrm{H}), 6.27(\mathrm{~d}, J=2.2 \mathrm{~Hz}, 1 \mathrm{H}), 5.80(\mathrm{~s}, 1 \mathrm{H}), 3.86(\mathrm{~s}, 3 \mathrm{H})$, $3.25(\mathrm{~s}, 3 \mathrm{H}), 2.71(\mathrm{~s}, 3 \mathrm{H}), 1.27(\mathrm{~s}, 9 \mathrm{H}) ;{ }^{13} \mathrm{C}$ NMR $\left(126 \mathrm{MHz}, \mathrm{CDCl}_{3}\right) \delta: 173.4,171.1,161.6,154.3,152.5(\mathrm{q}, J=$ $39.2 \mathrm{~Hz}$ ), 146.1, 143.9, 131.1, 126.4, 125.4, 122.5, 122.3, 119.0 (q, J = 275.8 Hz), 117.8, 109.2, 106.1, 96.2, 
80.3, 72.5, 66.7, 55.6, 28.3, 26.7, 26.1; ${ }^{19} \mathrm{~F}$ NMR (376 MHz, CDCl 3 ) ס: -70.80. HRMS (ESI-TOF): $\mathrm{m} / \mathrm{z}$ calcd. for $\mathrm{C}_{26} \mathrm{H}_{27} \mathrm{~N}_{4} \mathrm{NaO}_{5} \mathrm{~F}_{3}[\mathrm{M}+\mathrm{Na}]^{+}$: 555.1826; found: 555.1824. HPLC analysis: The enantiomeric excess was determined by HPLC with Chiralpak IC-H column (hexane $/ \mathrm{i}-\mathrm{PrOH}=80 / 20,0.8 \mathrm{~mL} \cdot \mathrm{min}^{-1}, \lambda=245 \mathrm{~nm}$ ): $t_{\text {major }}=$ $13.1 \mathrm{~min}, t_{\text {minor }}=28.8 \mathrm{~min}$.

tert-butyl ((3R,3'S)-7-chloro-1,1'-dimethyl-2,2'-dioxo-3'-((E)-(2,2,2-trifluoroethylidene)amino)-[3,3'-biindolin]-3-yl) carbamate $(3 r)$ : petroleum ether/ ethyl acetate $=10 / 1$

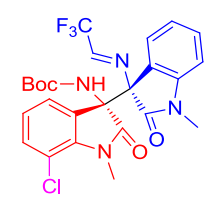

Yellow solid, 46.2mg, 86\% yield, >20:1 dr, 92\% ee, $[a]_{D}^{20}=+26.36(\mathrm{c}=0.22, \mathrm{MeOH})$.

${ }^{1} \mathrm{H}$ NMR $\left(400 \mathrm{MHz}, \mathrm{CDCl}_{3}\right) \delta: 7.46(\mathrm{~d}, J=2.0 \mathrm{~Hz}, 1 \mathrm{H}), 7.40-7.25(\mathrm{~m}, 3 \mathrm{H}), 7.17(\mathrm{~s}, 1 \mathrm{H}), 7.03(\mathrm{t}, J=7.6 \mathrm{~Hz}, 1 \mathrm{H})$, $6.84(\mathrm{dd}, J=18.0,7.8 \mathrm{~Hz}, 2 \mathrm{H}), 5.72(\mathrm{~s}, 1 \mathrm{H}), 3.26(\mathrm{~s}, 3 \mathrm{H}), 3.06(\mathrm{~s}, 3 \mathrm{H}), 1.27(\mathrm{~s}, 9 \mathrm{H}) ;{ }^{13} \mathrm{C} \mathrm{NMR}\left(101 \mathrm{MHz}, \mathrm{CDCl}_{3}\right)$ ס: 173.4, 170.8, 154.2, 152.9 (q, $J=39.1 \mathrm{~Hz}$ ), 143.9, 140.5, 132.2, 131.4, 128.7, 125.0, 124.2, 123.3, 122.7, $121.9,118.9$ (q, $J=275.5 \mathrm{~Hz}$ ), 115.7, 109.4, 80.7, 72.1, 66.7, 29.5, 28.2, 26.7; ${ }^{19} \mathrm{~F} \mathrm{NMR}\left(376 \mathrm{MHz}, \mathrm{CDCl}_{3}\right) \delta$ : -70.86. HRMS (ESI-TOF): $\mathrm{m} / z$ calcd. for $\mathrm{C}_{25} \mathrm{H}_{24} \mathrm{CIN}_{4} \mathrm{NaO}_{4} \mathrm{~F}_{3}[\mathrm{M}+\mathrm{Na}]^{+}:$559.1330; found: 559.1342. HPLC analysis: The enantiomeric excess was determined by HPLC with Chiralpak IC column (hexane/ $i$-PrOH $=$ 80/20, $\left.0.8 \mathrm{~mL} \cdot \mathrm{min}^{-1}, \lambda=254 \mathrm{~nm}\right): t_{\text {major }}=8.0 \mathrm{~min}, t_{\text {minor }}=14.2 \mathrm{~min}$.

tert-butyl ((3R,3'S)-1,1',7-trimethyl-2,2'-dioxo-3'-((E)-(2,2,2-trifluoroethylidene)amino)-[3,3'-biindolin]-3-yl) carbamate (3s): petroleum ether/ ethyl acetate $=5 / 1$

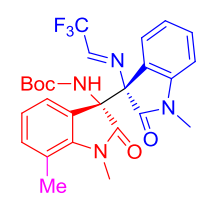

Yellow foam, $46.5 \mathrm{mg}, 90 \%$ yield, $>20: 1 \mathrm{dr}, 96 \%$ ee, $[a]_{D}^{20}=+34.55(\mathrm{c}=0.33, \mathrm{MeOH})$.

${ }^{1} \mathrm{H}$ NMR $\left(400 \mathrm{MHz}, \mathrm{CDCl}_{3}\right) \delta: 7.41(\mathrm{~d}, J=3.1 \mathrm{~Hz}, 1 \mathrm{H}), 7.30(\mathrm{td}, J=7.8,1.1 \mathrm{~Hz}, 1 \mathrm{H}), 7.18(\mathrm{~s}, 2 \mathrm{H}), 7.10(\mathrm{~d}, J=$ $7.6 \mathrm{~Hz}, 1 \mathrm{H}), 6.98(\mathrm{t}, J=7.5 \mathrm{~Hz}, 1 \mathrm{H}), 6.84(\mathrm{~d}, J=7.8 \mathrm{~Hz}, 1 \mathrm{H}), 6.75(\mathrm{t}, J=7.6 \mathrm{~Hz}, 1 \mathrm{H}), 5.61(\mathrm{~s}, 1 \mathrm{H}), 3.24(\mathrm{~s}, 3 \mathrm{H})$, $2.96(\mathrm{~s}, 3 \mathrm{H}), 2.41(\mathrm{~s}, 3 \mathrm{H}), 1.26(\mathrm{~s}, 9 \mathrm{H}) ;{ }^{13} \mathrm{C}$ NMR $\left(101 \mathrm{MHz}, \mathrm{CDCl}_{3}\right) \delta: 173.7,171.0,154.2,152.5$ (q, J = 39.2 $\mathrm{Hz}), 144.1,142.4,133.7,131.2,126.4,125.4,123.4,122.5,122.2,121.9,119.5,118.9$ (q, J = 275.9 Hz), 109.2, 80.3, 72.5, 66.6, 29.5, 28.2, 26.7, 19.1; ${ }^{19} \mathrm{~F}$ NMR (376 MHz, $\left.\mathrm{CDCl}_{3}\right) \delta:-70.79$. HRMS (ESI-TOF): $\mathrm{m} / z$ calcd. for $\mathrm{C}_{26} \mathrm{H}_{27} \mathrm{~N}_{4} \mathrm{NaO}_{4} \mathrm{~F}_{3}[\mathrm{M}+\mathrm{Na}]^{+}:$539.1877; found: 539.1880. HPLC analysis: The enantiomeric excess was determined by HPLC with Chiralpak IC column (hexane $/ \mathrm{i}-\mathrm{PrOH}=80 / 20,0.8 \mathrm{~mL} \cdot \mathrm{min}^{-1}, \lambda=254 \mathrm{~nm}$ ): $t_{\text {major }}=12.0$ $\min , t_{\text {minor }}=24.6 \mathrm{~min}$.

tert-butyl ((3R,3'S)-1'-methyl-2,2'-dioxo-1-phenyl-3'-((E)-(2,2,2-trifluoroethylidene)amino)-[3,3'-biindolin]-3-yl) carbamate (3t): petroleum ether/ ethyl acetate $=10 / 1$

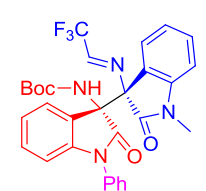

Yellow oil, $50.8 \mathrm{mg}, 90 \%$ yield, $>20: 1 \mathrm{dr}, 93 \%$ ee, $[a]_{D}^{20}=+51.40(\mathrm{c}=0.43, \mathrm{MeOH})$.

${ }^{1} \mathrm{H}$ NMR $\left(400 \mathrm{MHz}, \mathrm{CDCl}_{3}\right) \delta: 7.55(\mathrm{~d}, J=3.0 \mathrm{~Hz}, 1 \mathrm{H}), 7.52-7.43(\mathrm{~m}, 1 \mathrm{H}), 7.40-7.23(\mathrm{~m}, 6 \mathrm{H}), 7.15(\mathrm{t}, J=7.2 \mathrm{~Hz}$, $1 \mathrm{H}), 6.84(\mathrm{dd}, J=11.9,7.8 \mathrm{~Hz}, 2 \mathrm{H}), 6.75(\mathrm{~d}, J=5.4 \mathrm{~Hz}, 2 \mathrm{H}), 6.60(\mathrm{~d}, J=7.8 \mathrm{~Hz}, 1 \mathrm{H}), 5.83(\mathrm{~s}, 1 \mathrm{H}), 3.22(\mathrm{~s}, 3 \mathrm{H})$, 
1.30 (s, 9H); ${ }^{13} \mathrm{C}$ NMR (126 MHz, $\left.\mathrm{CDCl}_{3}\right) \delta: 172.6,171.0,154.5,152.7$ (q, $\left.J=39.2 \mathrm{~Hz}\right), 145.0,144.1,134.0$, $131.2,129.9,129.5,128.3,126.7,126.0,125.6,125.5,123.0,122.7,122.4,119.0$ (q, $J=275.8 \mathrm{~Hz}), 109.5$, 109.1, 80.5, 72.2, 67.1, 28.3, 26.7; ${ }^{19} \mathrm{~F}$ NMR (376 MHz, $\left.\mathrm{CDCl}_{3}\right)$ $\delta:-70.77$. HRMS (ESI-TOF): $\mathrm{m} / \mathrm{z}$ calcd. for $\mathrm{C}_{30} \mathrm{H}_{27} \mathrm{~N}_{4} \mathrm{NaO}_{4} \mathrm{~F}_{3}[\mathrm{M}+\mathrm{Na}]^{+}: 587.1877$; found: 587.1871. HPLC analysis: The enantiomeric excess was determined by HPLC with Chiralpak IC column (hexane $/ \mathrm{i}-\mathrm{PrOH}=80 / 20,0.8 \mathrm{~mL} \cdot \mathrm{min}^{-1}, \lambda=254 \mathrm{~nm}$ ): $t_{\text {major }}=7.7$ $\min , t_{\operatorname{minor}}=36.7 \mathrm{~min}$.

tert-butyl ((3R,3'S)-1'-methyl-2,2'-dioxo-1-propyl-3'-((E)-(2,2,2-trifluoroethylidene)amino)-[3,3'-biindolin]-3-yl) carbamate $(3 \mathbf{u})$ : petroleum ether/ ethyl acetate $=10 / 1$

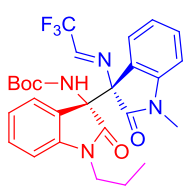

Yellow foam, $46.2 \mathrm{mg}, 87 \%$ yield, $>20: 1 d r,>99 \%$ ee, $[a]_{D}^{20}=+41.29(\mathrm{c}=0.31, \mathrm{MeOH})$.

${ }^{1} \mathrm{H}$ NMR $\left(400 \mathrm{MHz}, \mathrm{CDCl}_{3}\right) \delta: 7.48(\mathrm{q}, J=2.8 \mathrm{~Hz}, 1 \mathrm{H}), 7.41(\mathrm{~s}, 1 \mathrm{H}), 7.36(\mathrm{t}, J=7.7 \mathrm{~Hz}, 1 \mathrm{H}), 7.27(\mathrm{t}, J=7.7 \mathrm{~Hz}$, $1 \mathrm{H}), 7.18(\mathrm{~s}, 1 \mathrm{H}), 7.10(\mathrm{t}, J=7.5 \mathrm{~Hz}, 1 \mathrm{H}), 6.82(\mathrm{~d}, J=7.9 \mathrm{~Hz}, 1 \mathrm{H}), 6.70(\mathrm{t}, J=7.8 \mathrm{~Hz}, 2 \mathrm{H}), 5.65(\mathrm{~s}, 1 \mathrm{H})$, 3.57-3.38 (m, 1H), $3.25(\mathrm{~s}, 3 \mathrm{H}), 3.13-2.92(\mathrm{~m}, 1 \mathrm{H}), 1.23(\mathrm{~s}, 9 \mathrm{H}), 1.15-1.03(\mathrm{~m}, 1 \mathrm{H}), 0.85-0.73(\mathrm{~m}, 1 \mathrm{H}), 0.64(\mathrm{t}, J$ $=7.3 \mathrm{~Hz}, 3 \mathrm{H}) ;{ }^{13} \mathrm{C}$ NMR $\left(101 \mathrm{MHz}, \mathrm{CDCl}_{3}\right) \delta: 172.8,171.2,154.2,152.5$ (q, J=39.2 Hz), 144.3, 144.0, 131.0, 129.9, 126.0, 125.8, 125.6, 125.5, 122.7, 122.4, 118.9 (q, $J=275.7 \mathrm{~Hz}$ ), 109.1, 108.2, 80.3, 72.1, 66.7, 41.8, 28.2, 26.6, 20.3, 11.4; ${ }^{19} \mathrm{~F}$ NMR (376 MHz, $\mathrm{CDCl}_{3}$ ) $\delta:-70.82$. HRMS (ESI-TOF): $\mathrm{m} / z$ calcd. for $\mathrm{C}_{27} \mathrm{H}_{29} \mathrm{~N}_{4} \mathrm{NaO}_{4} \mathrm{~F}_{3}$ $\left[\mathrm{M}+\mathrm{Na}^{+}:\right.$553.2033, found: 553.2036. HPLC analysis: The enantiomeric excess was determined by HPLC with Chiralpak IC column (hexane $/ i-\mathrm{PrOH}=80 / 20,0.8 \mathrm{~mL} \cdot \mathrm{min}^{-1}, \lambda=285 \mathrm{~nm}$ ): $t_{\text {major }}=9.1 \mathrm{~min}, t_{\text {minor }}=22.0 \mathrm{~min}$.

tert-butyl ((3R,3'S)-1-benzyl-1'-methyl-2,2'-dioxo-3'-((E)-(2,2,2-trifluoroethylidene)amino)-[3,3'-biindolin]-3-yl) carbamate (3v): petroleum ether/ ethyl acetate $=10 / 1$

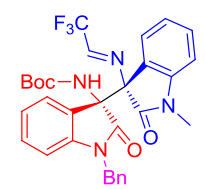

Yellow foam, $53.2 \mathrm{mg}, 92 \%$ yield, $>20: 1 \mathrm{dr},>99 \%$ ee, $[a]_{D}^{20}=+59.77(\mathrm{c}=0.43, \mathrm{MeOH})$.

${ }^{1} \mathrm{H}$ NMR $\left(400 \mathrm{MHz}, \mathrm{CDCl}_{3}\right) \delta: 7.50(\mathrm{~d}, J=2.0 \mathrm{~Hz}, 1 \mathrm{H}), 7.46(\mathrm{~s}, 1 \mathrm{H}), 7.31(\mathrm{t}, J=7.8 \mathrm{~Hz}, 1 \mathrm{H}), 7.24(\mathrm{t}, J=7.6 \mathrm{~Hz}$, $2 \mathrm{H}), 7.12(\mathrm{tt}, J=16.1,7.9 \mathrm{~Hz}, 4 \mathrm{H}), 6.86(\mathrm{~d}, J=7.9 \mathrm{~Hz}, 1 \mathrm{H}), 6.79-6.58(\mathrm{~m}, 3 \mathrm{H}), 6.53(\mathrm{~d}, J=7.8 \mathrm{~Hz}, 1 \mathrm{H}), 5.67(\mathrm{~s}$, $1 \mathrm{H}), 4.75(\mathrm{~d}, J=15.8 \mathrm{~Hz}, 1 \mathrm{H}), 4.33(\mathrm{~d}, J=15.2 \mathrm{~Hz}, 1 \mathrm{H}), 3.27(\mathrm{~s}, 3 \mathrm{H}), 1.26(\mathrm{~s}, 9 \mathrm{H}) ;{ }^{13} \mathrm{C} \mathrm{NMR}\left(101 \mathrm{MHz}, \mathrm{CDCl}_{3}\right)$ б: 173.5, 171.2, 154.3, 152.5 (q, $J=39.2 \mathrm{~Hz}$ ), 144.2, 143.9, 135.4, 131.1, 129.9, 128.7, 127.3, 127.0, 126.1, 125.7, 125.7, 123.1, 122.7, 122.4, 118.9 (q, $J=275.8 \mathrm{~Hz}$ ), 109.3, 109.1, 80.4, 72.1, 66.6, 44.4, 28.2, 26.7; ${ }^{19} \mathrm{~F}$ NMR (376 MHz, $\mathrm{CDCl}_{3}$ ) $\delta:-70.79$. HRMS (ESI-TOF): $\mathrm{m} / z$ calcd. for $\mathrm{C}_{31} \mathrm{H}_{29} \mathrm{~N}_{4} \mathrm{NaO}_{4} \mathrm{~F}_{3}[\mathrm{M}+\mathrm{Na}]^{+}: 601.2033$; found: 601.2023. HPLC analysis: The enantiomeric excess was determined by HPLC with Chiralpak IC column (hexane $/ i-\mathrm{PrOH}=80 / 20,0.8 \mathrm{~mL} \cdot \mathrm{min}^{-1}, \lambda=254 \mathrm{~nm}$ ): $t_{\text {major }}=9.0 \mathrm{~min}, t_{\text {minor }}=18.7 \mathrm{~min}$.

\section{d. General Procedure to Prepare Racemic Products 8a-8m}




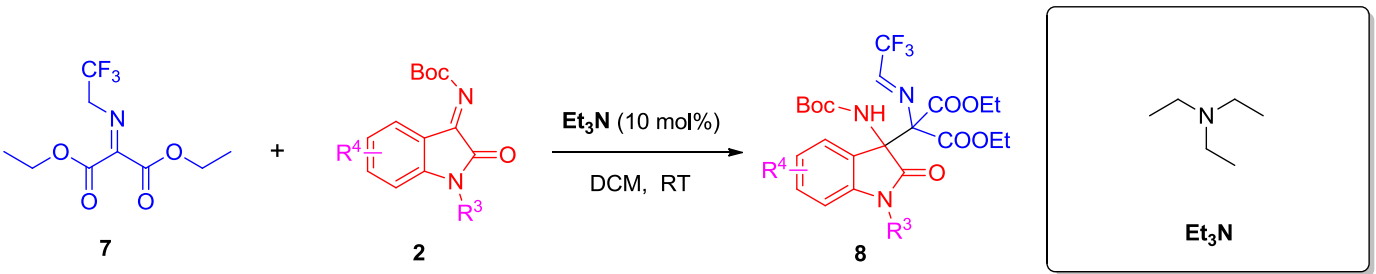

To a solution of isatin-derived $\mathrm{N}$-Boc ketimine $2(0.10 \mathrm{mmol}, 1.0$ equiv. $)$ and $\mathrm{Et}_{3} \mathrm{~N}(0.01 \mathrm{mmol}, 10 \mathrm{~mol} \%)$ in DCM (1 mL) was added diethyl 2-[(2,2,2-trifluoroethyl)-imino]malonate 7 (0.15 mmol, 1.5 equiv.). The mixture was stirred at room temperature until the reaction was completed (monitored by TLC analysis). The crude product was purified directly by flash column chromatography on silica gel (petroleum ether/ ethyl acetate = 10:1 5:1) to give the desired racemic products $8 \mathrm{a}-8 \mathrm{~m}$.

\section{e. General Procedure to Prepare Chiral Products $8 \mathrm{a}-8 \mathrm{~m}$}
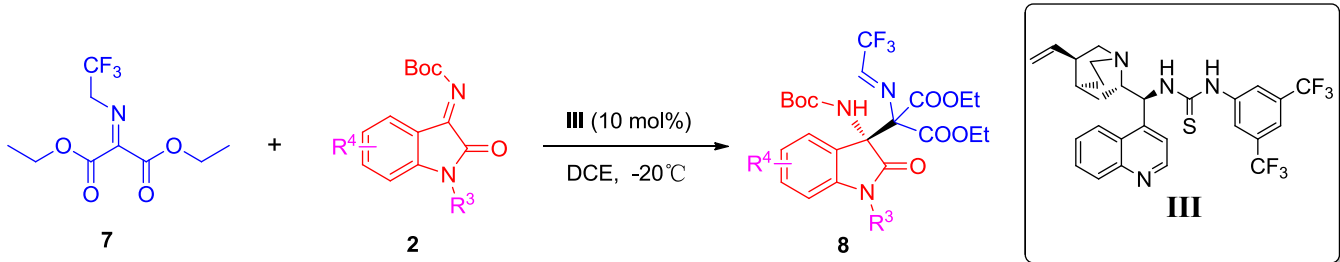

To a solution of isatin-derived $\mathrm{N}$-Boc ketimine $2(0.10 \mathrm{mmol}, 1.0$ equiv. $)$ and catalyst III $(0.01 \mathrm{mmol}, 10$ $\mathrm{mol} \%$ ) in DCE (1 mL) was added diethyl 2-[(2,2,2-trifluoroethyl)-imino]malonate 7 (0.15 mmol, 1.5 equiv.). The mixture was stirred at $-20^{\circ} \mathrm{C}$ until the reaction was completed (monitored by TLC analysis). The crude product was purified directly by flash column chromatography on silica gel (petroleum ether/ ethyl acetate $=10: 1 \sim 5: 1$ ) to give the desired chiral products $8 \mathbf{a}-8 \mathrm{~m}$.

\section{f. Analytical Data for Products 8a-8m}

( $R, E)$-diethyl 2-(3-((tert-butoxycarbonyl)amino)-1-methyl-2-oxoindolin-3-yl)-2-((2,2,2-trifluoroethylidene)amino) malonate $(8 a)$ : petroleum ether/ ethyl acetate $=5 / 1$<smiles>CCOC(=O)NC(N=CC(F)(F)F)(C(=O)OCC)C1(C(=O)OCC)C(=O)N(C)c2ccccc21</smiles>

White foam, $47.9 \mathrm{mg}, 93 \%$ yield, $>20: 1 \mathrm{dr}$, >99\% ee, $[a]_{D}^{20}=+45.26(\mathrm{c}=0.23, \mathrm{MeOH})$.

${ }^{1} \mathrm{H}$ NMR $\left(400 \mathrm{MHz}, \mathrm{CDCl}_{3}\right) \delta: 7.70(\mathrm{~d}, J=2.8 \mathrm{~Hz}, 1 \mathrm{H}), 7.49(\mathrm{~d}, J=7.4 \mathrm{~Hz}, 1 \mathrm{H}), 7.29(\mathrm{t}, J=7.7 \mathrm{~Hz}, 1 \mathrm{H}), 6.98(\mathrm{t}$, $J=7.4 \mathrm{~Hz}, 1 \mathrm{H}), 6.76(\mathrm{~d}, J=7.8 \mathrm{~Hz}, 1 \mathrm{H}), 6.25(\mathrm{~s}, 1 \mathrm{H}), 4.42-4.32(\mathrm{~m}, 2 \mathrm{H}), 4.08(\mathrm{~d}, J=5.5 \mathrm{~Hz}, 2 \mathrm{H}), 3.21(\mathrm{~s}, 3 \mathrm{H})$, $1.33(\mathrm{t}, J=7.1 \mathrm{~Hz}, 3 \mathrm{H}), 1.22(\mathrm{~s}, 9 \mathrm{H}), 1.12(\mathrm{t}, J=6.6 \mathrm{~Hz}, 3 \mathrm{H}) ;{ }^{13} \mathrm{C}$ NMR $\left(101 \mathrm{MHz}, \mathrm{CDCl}_{3}\right) \delta: 173.1,165.3,163.7$, 156.1 (q, $J=39.3 \mathrm{~Hz}$ ), 153.6, 145.6, 129.9, 126.9, 125.5, 122.6, 118.8 (q, $J=245.7 \mathrm{~Hz}$ ), 107.8, 80.4, 63.7, 63.5 28.2, 26.5, 13.9, 13.7; ${ }^{19} \mathrm{~F}$ NMR (376 MHz, $\mathrm{CDCl}_{3}$ ) $\delta:-72.32$. HRMS (ESI-TOF): $\mathrm{m} / \mathrm{z}[\mathrm{M}+\mathrm{H}]^{+}$calcd. for $\left[\mathrm{C}_{23} \mathrm{H}_{29} \mathrm{~F}_{3} \mathrm{~N}_{3} \mathrm{O}_{7}\right]^{+}:$516.1952, found: 516.1964; HPLC analysis: The enantiomeric excess was determined by HPLC with a Chiralpak IA column (hexane $/ i-\mathrm{PrOH}=95 / 5,1.0 \mathrm{~mL} \cdot \mathrm{min}^{-1}, \lambda=254 \mathrm{~nm}$ ): $t_{\text {major }}=7.8 \mathrm{~min}, t_{\text {minor }}=9.0$ $\min$.

$(R, E)$-diethyl 2-(3-((tert-butoxycarbonyl)amino)-5-fluoro-1-methyl-2-oxoindolin-3-yl)-2-((2,2,2-trifluoroethylidene) 
amino)malonate $(\mathbf{8 b})$ : petroleum ether/ ethyl acetate $=5 / 1$

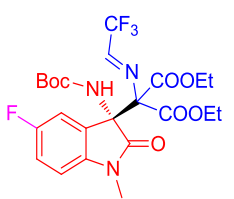

White foam, $50.7 \mathrm{mg}, 95 \%$ yield, $>20: 1 \mathrm{dr},>99 \%$ ee, $[a]_{D}^{20}=+40.81(\mathrm{c}=0.43, \mathrm{MeOH})$.

${ }^{1} \mathrm{H}$ NMR $\left(400 \mathrm{MHz}, \mathrm{CDCl}_{3}\right) \delta: 7.73(\mathrm{~s}, 1 \mathrm{H}), 7.29(\mathrm{~d}, J=7.5 \mathrm{~Hz}, 1 \mathrm{H}), 6.99(\mathrm{t}, J=8.4 \mathrm{~Hz}, 1 \mathrm{H}), 6.74-6.64(\mathrm{~m}, 1 \mathrm{H})$, $6.26(\mathrm{~s}, 1 \mathrm{H}), 4.38(\mathrm{~s}, 2 \mathrm{H}), 4.12(\mathrm{~d}, J=0.7 \mathrm{~Hz}, 2 \mathrm{H}), 3.20(\mathrm{~s}, 3 \mathrm{H}), 1.32(\mathrm{t}, J=6.4 \mathrm{~Hz}, 3 \mathrm{H}), 1.25(\mathrm{~s}, 9 \mathrm{H}), 1.16(\mathrm{~s}$, $3 \mathrm{H}) ;{ }^{13} \mathrm{C}$ NMR $\left(101 \mathrm{MHz}, \mathrm{CDCl}_{3}\right) \delta: 172.9,164.2(\mathrm{~d}, J=207.2 \mathrm{~Hz}), 159.1(\mathrm{~d}, J=240.1 \mathrm{~Hz}), 156.3(\mathrm{q}, J=39.3$ $\mathrm{Hz}$ ), 153.5, 141.8, 128.4 (d, $J=1.2 \mathrm{~Hz}), 128.4(\mathrm{~d}, J=5.2 \mathrm{~Hz}), 118.5$ (q, J = 275.8 Hz), 116.0 (d, J = 23.6 Hz), $113.9(\mathrm{~d}, J=25.8 \mathrm{~Hz}), 108.2(\mathrm{~d}, J=8.0 \mathrm{~Hz}), 80.6,63.9,63.6,28.2,26.7,13.9,13.7 ;{ }^{19} \mathrm{~F} \mathrm{NMR}\left(376 \mathrm{MHz}, \mathrm{CDCl}_{3}\right)$ ס: $-72.37,-120.42$. HRMS (ESI-TOF): $m / z[\mathrm{M}+\mathrm{H}]^{+}$calcd. for $\left[\mathrm{C}_{23} \mathrm{H}_{28} \mathrm{~F}_{4} \mathrm{~N}_{3} \mathrm{O}_{7}\right]^{+}: 534.1858$, found: 534.1847 ; HPLC analysis: The enantiomeric excess was determined by HPLC with a Chiralpak IA column (hexane/ $i-\mathrm{PrOH}=$ $\left.95 / 5,1.0 \mathrm{~mL} \cdot \mathrm{min}^{-1}, \lambda=254 \mathrm{~nm}\right): t_{\text {major }}=8.3 \mathrm{~min}, t_{\text {minor }}=9.6 \mathrm{~min}$.

$(R, E)$-diethyl 2-(3-((tert-butoxycarbonyl)amino)-5-chloro-1-methyl-2-oxoindolin-3-yl)-2-((2,2,2-trifluoroethyliden e)amino)malonate $(\mathbf{8 c})$ : petroleum ether/ ethyl acetate $=5 / 1$

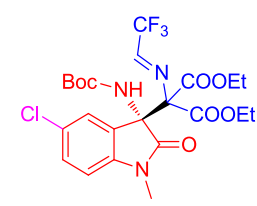

White solid, $50.6 \mathrm{mg}, 92 \%$ yield, $>20: 1 \mathrm{dr},>99 \%$ ee, $[a]_{D}^{20}=+45.57(\mathrm{c}=0.37, \mathrm{MeOH})$.

${ }^{1} \mathbf{H}$ NMR $\left(400 \mathrm{MHz}, \mathrm{CDCl}_{3}\right) \delta: 7.73(\mathrm{q}, J=2.8 \mathrm{~Hz}, 1 \mathrm{H}), 7.48(\mathrm{~d}, J=2.0 \mathrm{~Hz}, 1 \mathrm{H}), 7.29-7.23(\mathrm{~m}, 1 \mathrm{H}), 6.69(\mathrm{~d}, J=$ $8.3 \mathrm{~Hz}, 1 \mathrm{H}), 6.27(\mathrm{~s}, 1 \mathrm{H}), 4.42-4.33(\mathrm{~m}, 2 \mathrm{H}), 4.25-4.05(\mathrm{~m}, 2 \mathrm{H}), 3.19(\mathrm{~s}, 3 \mathrm{H}), 1.32(\mathrm{t}, J=7.1 \mathrm{~Hz}, 3 \mathrm{H}), 1.25(\mathrm{~s}$, $9 \mathrm{H}), 1.16(\mathrm{~s}, 3 \mathrm{H}) ;{ }^{13} \mathrm{C}$ NMR $\left(101 \mathrm{MHz}, \mathrm{CDCl}_{3}\right) \delta: 172.8,164.9,163.3,156.4$ (q, $\left.J=39.3 \mathrm{~Hz}\right), 153.5,144.4$, 129.8, 128.6, 127.8, 125.9, 118.5 (q, $J=275.7 \mathrm{~Hz}$ ), 108.7, 80.7, 63.9, 63.7, 28.2, 26.6, 13.9, 13.7; ${ }^{19} \mathrm{~F} \mathrm{NMR}$ (376 MHz, CDCl 3 ) ס: -72.38. HRMS (ESI-TOF): $\mathrm{m} / \mathrm{z}[\mathrm{M}+\mathrm{H}]^{+}$calcd. for $\left[\mathrm{C}_{23} \mathrm{H}_{28} \mathrm{~F}_{3} \mathrm{CIN}_{3} \mathrm{O}_{7}\right]^{+}: 550.1562$, found: 550.1566. HPLC analysis: The enantiomeric excess was determined by HPLC with a Chiralpak IA column (hexane $/ \mathrm{i}-\mathrm{PrOH}=95 / 5,1.0 \mathrm{~mL} \cdot \mathrm{min}^{-1}, \lambda=254 \mathrm{~nm}$ ): $t_{\text {major }}=8.2 \mathrm{~min}, t_{\text {minor }}=9.3 \mathrm{~min}$.

(R,E)-diethyl 2-(5-bromo-3-((tert-butoxycarbonyl)amino)-1-methyl-2-oxoindolin-3-yl)-2-((2,2,2-trifluoroethyliden e)amino)malonate $(\mathbf{8 d})$ : petroleum ether/ ethyl acetate $=5 / 1$

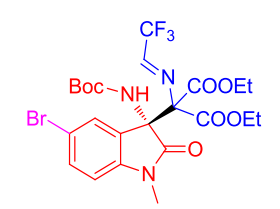

White solid, $55.3 \mathrm{mg}, 93 \%$ yield, $>20: 1 \mathrm{dr},>99 \%$ ee, $[a]_{D}^{20}=+40.42(\mathrm{c}=0.19$, MeOH).

${ }^{1} \mathrm{H}$ NMR $\left(400 \mathrm{MHz}, \mathrm{CDCl}_{3}\right) \delta: 7.74(\mathrm{q}, J=2.9 \mathrm{~Hz}, 1 \mathrm{H}), 7.61(\mathrm{~d}, J=1.9 \mathrm{~Hz}, 1 \mathrm{H}), 7.42(\mathrm{dd}, J=8.3,2.0 \mathrm{~Hz}, 1 \mathrm{H})$, $6.65(\mathrm{~d}, J=8.3 \mathrm{~Hz}, 1 \mathrm{H}), 6.27(\mathrm{~s}, 1 \mathrm{H}), 4.42-4.33(\mathrm{~m}, 2 \mathrm{H}), 4.25-4.07(\mathrm{~m}, 2 \mathrm{H}), 3.19(\mathrm{~s}, 3 \mathrm{H}), 1.33(\mathrm{t}, J=7.1 \mathrm{~Hz}, 3 \mathrm{H})$, $1.26(\mathrm{~s}, 9 \mathrm{H}), 1.18(\mathrm{~s}, 3 \mathrm{H}) ;{ }^{13} \mathrm{C}$ NMR $\left(101 \mathrm{MHz} \mathrm{CDCl}_{3}\right) \delta: 172.7,164.9,163.4,156.4$ (q, J = 39.4 Hz), 153.5, 144.8, 132.7, 129.0, 128.6, 118.5 (q, $J=275.6 \mathrm{~Hz}$ ), 115.1, 109.3, 80.8, 64.0, 63.7, 28.2, 26.6, 14.0, 13.8; ${ }^{19} \mathrm{~F}$ NMR (376 MHz, $\mathrm{CDCl}_{3}$ ) $\delta:-72.38$. HRMS (ESI-TOF): $\mathrm{m} / z[\mathrm{M}+\mathrm{H}]^{+}$calcd. for $\left[\mathrm{C}_{23} \mathrm{H}_{28} \mathrm{~F}_{3} \mathrm{BrN}_{3} \mathrm{O}_{7}\right]^{+}: 594.1057$, found: 594.1072; HPLC analysis: The enantiomeric excess was determined by HPLC with a Chiralpak IA column (hexane $/ \mathrm{i}-\mathrm{PrOH}=95 / 5,1.0 \mathrm{~mL} \cdot \mathrm{min}^{-1}, \lambda=254 \mathrm{~nm}$ ): $t_{\mathrm{major}}=8.7 \mathrm{~min}, t_{\text {minor }}=9.8 \mathrm{~min}$. 
(R,E)-diethyl 2-(3-((tert-butoxycarbonyl)amino)-5-methoxy-1-methyl-2-oxoindolin-3-yl)-2-((2,2,2-trifluoroethylid ene)amino)malonate (8e): petroleum ether/ ethyl acetate $=5 / 1$

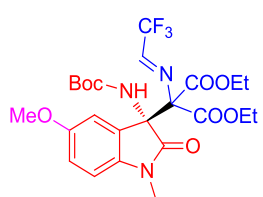

colourless oil, $50.2 \mathrm{mg}, 92 \%$ yield, $>20: 1 \mathrm{dr}, 97 \%$ ee, $[a]_{D}^{20}=+51.17(\mathrm{c}=0.35, \mathrm{MeOH})$.

${ }^{1} \mathrm{H}$ NMR $\left(400 \mathrm{MHz}, \mathrm{CDCl}_{3}\right) \delta: 7.70(\mathrm{~d}, J=2.7 \mathrm{~Hz}, 1 \mathrm{H}), 7.16(\mathrm{~d}, J=2.4 \mathrm{~Hz}, 1 \mathrm{H}), 6.81(\mathrm{dd}, J=8.5,2.6 \mathrm{~Hz}, 1 \mathrm{H})$, $6.66(\mathrm{~d}, J=8.5 \mathrm{~Hz}, 1 \mathrm{H}$ ), 6.24 (s, 1H), 4.36 (ddq, $J=14.2,7.1,3.6 \mathrm{~Hz}, 2 \mathrm{H}), 4.08$ (s, 2H), 3.73 (s, 3H), 3.18 (s, $3 \mathrm{H}), 1.32(\mathrm{t}, J=7.1 \mathrm{~Hz}, 3 \mathrm{H}), 1.23(\mathrm{~s}, 9 \mathrm{H}), 1.12(\mathrm{~s}, 3 \mathrm{H}) ;{ }^{13} \mathrm{C}$ NMR $\left(101 \mathrm{MHz}, \mathrm{CDCl}_{3}\right) \delta:$ 172.8, 165.3, 163.5, 156.1 (q, $J=39.3 \mathrm{~Hz}$ ), 155.8, 153.5, 139.1, 127.9, 118.6 (q, $J=275.5 \mathrm{~Hz}$ ), 114.4, 112.7, 108.1, 80.4, 63.7, 63.4, 55.7, 28.2, 26.6, 13.9, 13.7; ${ }^{19} \mathrm{~F}$ NMR (376 MHz, $\mathrm{CDCl}_{3}$ ) $\delta:-72.32$. HRMS (ESI-TOF): $\mathrm{m} / \mathrm{z}[\mathrm{M}+\mathrm{H}]^{+}$calcd. for $\left[\mathrm{C}_{24} \mathrm{H}_{31} \mathrm{~F}_{3} \mathrm{~N}_{3} \mathrm{O}_{8}\right]^{+}: 546.2058$, found: 546.2061; HPLC analysis: The enantiomeric excess was determined by HPLC with a Chiralpak IA column (hexane $/ \mathrm{i}-\mathrm{PrOH}=95 / 5,1.0 \mathrm{~mL} \cdot \mathrm{min}^{-1}, \lambda=280 \mathrm{~nm}$ ): $t_{\text {major }}=10.5 \mathrm{~min}, t_{\text {minor }}=$ $12.0 \mathrm{~min}$.

(R,E)-diethyl 2-(3-((tert-butoxycarbonyl)amino)-1,5-dimethyl-2-oxoindolin-3-yl)-2-((2,2,2-trifluoroethylidene) amino)malonate (8f): petroleum ether/ ethyl acetate $=5 / 1$

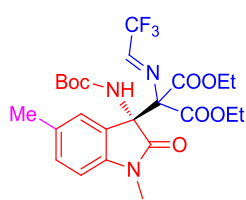

colourless oil, $45.5 \mathrm{mg}, 86 \%$ yield, $>20: 1 \mathrm{dr}, 98 \%$ ee, $[a]_{D}^{20}=+46.63(\mathrm{c}=0.35, \mathrm{MeOH})$.

${ }^{1} \mathrm{H}$ NMR $\left(400 \mathrm{MHz}, \mathrm{CDCl}_{3}\right) \delta: 7.70(\mathrm{~d}, J=2.8 \mathrm{~Hz}, 1 \mathrm{H}), 7.28(\mathrm{~s}, 1 \mathrm{H}), 7.07(\mathrm{~d}, J=7.8 \mathrm{~Hz}, 1 \mathrm{H}), 6.64(\mathrm{~d}, J=7.9 \mathrm{~Hz}$, $1 \mathrm{H}), 6.24$ (s, 1H), 4.36 (ddq, $J=13.9,6.8,3.6 \mathrm{~Hz}, 2 \mathrm{H}$ ), 4.09 (d, $J=5.2 \mathrm{~Hz}, 2 \mathrm{H}$ ), $3.18(\mathrm{~s}, 3 \mathrm{H}), 2.27(\mathrm{~s}, 3 \mathrm{H}), 1.32$ $(\mathrm{t}, J=7.1 \mathrm{~Hz}, 3 \mathrm{H}), 1.22(\mathrm{~s}, 9 \mathrm{H}), 1.15-1.07(\mathrm{~m}, 3 \mathrm{H}) ;{ }^{13} \mathrm{C} \mathrm{NMR}\left(101 \mathrm{MHz}, \mathrm{CDCl}_{3}\right)$ ס: 173.0, 165.3, 163.7, 156.1 (q, $J=39.1 \mathrm{~Hz}$ ), 153.6, 143.1, 132.0, 130.2, 126.8, 126.2, 118.6 (q, $J=275.7 \mathrm{~Hz}), 107.5,80.3,63.6,63.4,28.2$, 26.5, 21.3, 13.9, 13.7; ${ }^{19} \mathrm{~F}$ NMR (376 $\mathrm{MHz}, \mathrm{CDCl}_{3}$ ) $\delta:-72.31$. HRMS (ESI-TOF): $\mathrm{m} / \mathrm{z}[\mathrm{M}+\mathrm{H}]^{+}$calcd. for $\left[\mathrm{C}_{24} \mathrm{H}_{31} \mathrm{~F}_{3} \mathrm{~N}_{3} \mathrm{O}_{7}\right]^{+}: 530.2109$, found: 530.2120 ; HPLC analysis: The enantiomeric excess was determined by HPLC with a Chiralpak IA column (hexane $/ i-\mathrm{PrOH}=95 / 5,1.0 \mathrm{~mL} \cdot \mathrm{min}^{-1}, \lambda=254 \mathrm{~nm}$ ) $: t_{\text {major }}=8.4 \mathrm{~min}, t_{\text {minor }}=9.9$ $\min$.

$(R, E)$-diethyl 2-(3-((tert-butoxycarbonyl)amino)-6-chloro-1-methyl-2-oxoindolin-3-yl)-2-((2,2,2-trifluoroethyliden e)amino)malonate $(\mathbf{8 g})$ : petroleum ether/ ethyl acetate $=5 / 1$

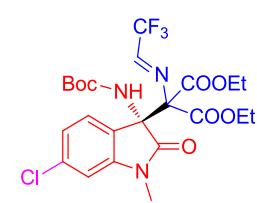

colourless foam, $52.8 \mathrm{mg}, 96 \%$ yield, $>20: 1 \mathrm{dr},>99 \%$ ee, $[a]_{D}^{20}=+38.22(\mathrm{c}=0.46, \mathrm{MeOH})$.

${ }^{1} \mathrm{H}$ NMR $\left(400 \mathrm{MHz}, \mathrm{CDCl}_{3}\right) \delta: 7.72(\mathrm{dd}, J=5.5,2.7 \mathrm{~Hz}, 1 \mathrm{H}), 7.42(\mathrm{~d}, J=8.0 \mathrm{~Hz}, 1 \mathrm{H}), 6.95(\mathrm{dd}, J=8.0,1.8 \mathrm{~Hz}$, $1 \mathrm{H}), 6.76(\mathrm{~d}, J=1.8 \mathrm{~Hz}, 1 \mathrm{H}), 6.23(\mathrm{~s}, 1 \mathrm{H}), 4.41-4.32(\mathrm{~m}, 2 \mathrm{H}), 4.10(\mathrm{~s}, 2 \mathrm{H}), 3.19(\mathrm{~s}, 3 \mathrm{H}), 1.32(\mathrm{t}, J=7.1 \mathrm{~Hz}, 4 \mathrm{H})$, $1.24(\mathrm{~s}, 9 \mathrm{H}), 1.14(\mathrm{~s}, 3 \mathrm{H}) ;{ }^{13} \mathrm{C}$ NMR $\left(101 \mathrm{MHz}, \mathrm{CDCl}_{3}\right) \delta: 173.1,165.1,163.3,156.3$ (q, J=39.5 Hz), 153.5, 146.9, 135.8, 126.5, 125.3, 122.4, 118.5 (q, $J=255.5 \mathrm{~Hz}$ ), 108.6, 80.7, 63.8, 63.6, 28.2, 26.6, 13.9, 13.7; ${ }^{19} \mathrm{~F}$ NMR (376 MHz, $\mathrm{CDCl}_{3}$ ) $\delta:-72.30$. HRMS (ESI-TOF): $\mathrm{m} / z[\mathrm{M}+\mathrm{H}]^{+}$calcd. for $\left[\mathrm{C}_{23} \mathrm{H}_{28} \mathrm{~F}_{3} \mathrm{CIN}_{3} \mathrm{O}_{7}\right]^{+}: 550.1562$, found: 550.1574; HPLC analysis: The enantiomeric excess was determined by HPLC with a Chiralpak IB column 
(hexane $/ \mathrm{i}-\mathrm{PrOH}=96 / 4,1.0 \mathrm{~mL} \cdot \mathrm{min}^{-1}, \lambda=254 \mathrm{~nm}$ ): $t_{\text {major }}=7.0 \mathrm{~min}, t_{\text {minor }}=8.6 \mathrm{~min}$.

(R,E)-diethyl 2-(3-((tert-butoxycarbonyl)amino)-6-methoxy-1-methyl-2-oxoindolin-3-yl)-2-((2,2,2-trifluoroethylid ene)amino)malonate (8h): petroleum ether/ ethyl acetate $=5 / 1$

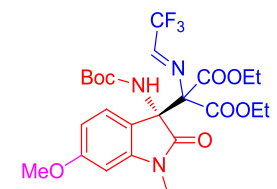

colourless oil, $50.2 \mathrm{mg}, 92 \%$ yield, $>20: 1 \mathrm{dr},>99 \%$ ee, $[a]_{D}^{20}=+37.54(\mathrm{c}=0.37, \mathrm{MeOH})$.

${ }^{1} \mathrm{H}$ NMR $\left(500 \mathrm{MHz}, \mathrm{CDCl}_{3}\right) \delta: 7.69(\mathrm{~s}, 1 \mathrm{H}), 7.40(\mathrm{~d}, J=8.1 \mathrm{~Hz}, 1 \mathrm{H}), 6.46(\mathrm{~d}, J=8.3 \mathrm{~Hz}, 1 \mathrm{H}), 6.34(\mathrm{~s}, 1 \mathrm{H}), 6.21$ (s, 1H), 4.44-4.28 (m, 2H), $4.08(\mathrm{~s}, 2 \mathrm{H}), 3.80(\mathrm{~s}, 3 \mathrm{H}), 3.19(\mathrm{~s}, 3 \mathrm{H}), 1.33(\mathrm{t}, J=7.0 \mathrm{~Hz}, 3 \mathrm{H}), 1.25(\mathrm{~s}, 9 \mathrm{H}), 1.13(\mathrm{~s}$, $3 \mathrm{H}) ;{ }^{13} \mathrm{C}$ NMR $\left(126 \mathrm{MHz}, \mathrm{CDCl}_{3}\right) \delta: 173.5,165.3,163.6,161.4,155.8$ (q, J = 38.7 Hz), 153.5, 146.8, 126.4, $118.5,118.5$ (q, $J=275.6 \mathrm{~Hz}), 105.9,95.9,80.2,63.5,63.3,55.4,28.1,26.4,13.8,13.6 ;{ }^{19} \mathrm{~F} \mathrm{NMR}(376 \mathrm{MHz}$, $\mathrm{CDCl}_{3}$ ) ठ: -72.23. HRMS (ESI-TOF): $\mathrm{m} / \mathrm{z}[\mathrm{M}+\mathrm{H}]^{+}$calcd. for $\left[\mathrm{C}_{24} \mathrm{H}_{31} \mathrm{~F}_{3} \mathrm{~N}_{3} \mathrm{O}_{8}\right]^{+}: 546.2058$, found: 546.2064; HPLC analysis: The enantiomeric excess was determined by HPLC with a Chiralpak IA column (hexane/ $i-\mathrm{PrOH}=$ $\left.95 / 5,1.0 \mathrm{~mL} \cdot \mathrm{min}^{-1}, \lambda=254 \mathrm{~nm}\right): t_{\text {major }}=7.8 \mathrm{~min}, t_{\text {minor }}=9.1 \mathrm{~min}$.

(R,E)-diethyl 2-(3-((tert-butoxycarbonyl)amino)-7-chloro-1-methyl-2-oxoindolin-3-yl)-2-((2,2,2-trifluoroethyliden e)amino)malonate $(\mathbf{8 i})$ : petroleum ether/ ethyl acetate $=5 / 1$

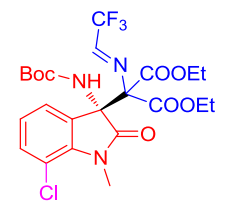

colourless oil, $52.8 \mathrm{mg}, 96 \%$ yield, $>20: 1 \mathrm{dr}, 97 \%$ ee, $[a]_{D}^{20}=+26.23(\mathrm{c}=0.22, \mathrm{MeOH})$.

${ }^{1} \mathbf{H}$ NMR $\left(400 \mathrm{MHz}, \mathrm{CDCl}_{3}\right) \delta: 7.73(\mathrm{dd}, J=5.6,2.7 \mathrm{~Hz}, 1 \mathrm{H}), 7.42(\mathrm{~d}, J=7.4 \mathrm{~Hz}, 1 \mathrm{H}), 7.21(\mathrm{~d}, J=8.2 \mathrm{~Hz}, 1 \mathrm{H})$, $6.89(\mathrm{t}, J=7.8 \mathrm{~Hz}, 1 \mathrm{H}), 6.19(\mathrm{~s}, 1 \mathrm{H}), 4.42-4.33(\mathrm{~m}, 2 \mathrm{H}), 4.21-4.00(\mathrm{~m}, 2 \mathrm{H}), 3.60(\mathrm{~s}, 3 \mathrm{H}), 1.33(\mathrm{t}, J=7.1 \mathrm{~Hz}, 3 \mathrm{H})$, 1.25 (s, 9H), 1.14 (t, $J=6.9 \mathrm{~Hz}, 3 \mathrm{H}) ;{ }^{13} \mathrm{C}$ NMR $\left(101 \mathrm{MHz}, \mathrm{CDCl}_{3}\right) \delta: 173.7,165.1,163.3,156.8(\mathrm{q}, J=39.3 \mathrm{~Hz})$, 153.5, 141.5, 132.2, 129.9, 123.9, 123.4, 118.6 (q, $J=275.5 \mathrm{~Hz}$ ), 115.3, 80.8, 63.9, 63.7, 30.1, 28.2, 13.9, 13.7;

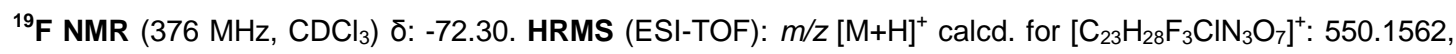
found: 550.1570; HPLC analysis: The enantiomeric excess was determined by HPLC with a Chiralpak IA column (hexane $/ \mathrm{i}-\mathrm{PrOH}=97 / 3,1.0 \mathrm{~mL} \cdot \mathrm{min}^{-1}, \lambda=230 \mathrm{~nm}$ ) $: t_{\text {major }}=8.0 \mathrm{~min}, t_{\text {minor }}=8.9 \mathrm{~min}$.

$(R, E)$-diethyl 2-(3-((tert-butoxycarbonyl)amino)-1,7-dimethyl-2-oxoindolin-3-yl)-2-((2,2,2-trifluoroethylidene) amino)malonate $(8 \mathbf{j})$ : petroleum ether/ ethyl acetate $=5 / 1$

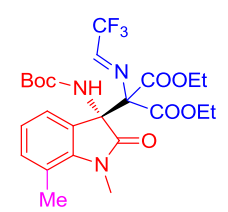

colourless oil, $51.9 \mathrm{mg}, 98 \%$ yield, $>20: 1 \mathrm{dr},>99 \%$ ee, $[a]_{D}^{20}=+39.24(\mathrm{c}=0.25, \mathrm{MeOH})$.

${ }^{1} \mathrm{H}$ NMR $\left(400 \mathrm{MHz}, \mathrm{CDCl}_{3}\right) \delta: 7.66(\mathrm{~d}, J=2.3 \mathrm{~Hz}, 1 \mathrm{H}), 7.33(\mathrm{~d}, J=7.4 \mathrm{~Hz}, 1 \mathrm{H}), 7.00(\mathrm{~d}, J=7.7 \mathrm{~Hz}, 1 \mathrm{H}), 6.85(\mathrm{t}$, $J=7.6 \mathrm{~Hz}, 1 \mathrm{H}), 6.28(\mathrm{~s}, 1 \mathrm{H}), 4.42-4.32(\mathrm{~m}, 2 \mathrm{H}), 4.23-4.02(\mathrm{~m}, 2 \mathrm{H}), 3.49(\mathrm{~s}, 3 \mathrm{H}), 2.52(\mathrm{~s}, 3 \mathrm{H}), 1.32(\mathrm{t}, J=7.1 \mathrm{~Hz}$ $3 \mathrm{H}), 1.24(\mathrm{~s}, 9 \mathrm{H}), 1.14(\mathrm{t}, J=6.9 \mathrm{~Hz}, 3 \mathrm{H}) ;{ }^{13} \mathrm{C}$ NMR $\left(101 \mathrm{MHz}, \mathrm{CDCl}_{3}\right) \delta: 173.9,165.3,163.7,156.1$ (q, $J=38.5$ $\mathrm{Hz}$ ), 153.5, 143.2, 133.8, 127.4, 123.2, 122.5, 119.2, 118.6 (q, $J=276.0 \mathrm{~Hz}$ ), 80.3, 63.6, 63.4, 30.1, 28.2, 19.3, 13.9, 13.7; ${ }^{19} \mathrm{~F}$ NMR (376 MHz, CDCl 3 ) $\delta:-72.35$. HRMS (ESI-TOF): $\mathrm{m} / z[\mathrm{M}+\mathrm{H}]^{+}$calcd. for $\left[\mathrm{C}_{24} \mathrm{H}_{31} \mathrm{~F}_{3} \mathrm{~N}_{3} \mathrm{O}_{7}\right]^{+}$: 530.2109, found: 530.2114; HPLC analysis: The enantiomeric excess was determined by HPLC with a 
Chiralpak IA column (hexane $/ \mathrm{i}-\mathrm{PrOH}=95 / 5,1.0 \mathrm{~mL} \cdot \mathrm{min}^{-1}, \lambda=280 \mathrm{~nm}$ ): $t_{\text {major }}=8.1 \mathrm{~min}, t_{\text {minor }}=7.2 \mathrm{~min}$.

(R,E)-diethyl 2-(3-((tert-butoxycarbonyl)amino)-2-oxo-1-propylindolin-3-yl)-2-((2,2,2-trifluoroethylidene)amino) malonate (8k): petroleum ether/ ethyl acetate $=10 / 1$

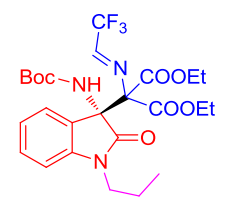

colourless oil, $48.9 \mathrm{mg}, 90 \%$ yield, $>20: 1 \mathrm{dr}, 97 \%$ ee, $[a]_{D}^{20}=+32.42(\mathrm{c}=0.21, \mathrm{MeOH})$.

${ }^{1} \mathrm{H}$ NMR $\left(400 \mathrm{MHz}, \mathrm{CDCl}_{3}\right) \delta: 7.67(\mathrm{~d}, J=2.6 \mathrm{~Hz}, 1 \mathrm{H}), 7.51(\mathrm{~d}, J=7.4 \mathrm{~Hz}, 1 \mathrm{H}), 7.27(\mathrm{t}, J=7.2 \mathrm{~Hz}, 1 \mathrm{H}), 6.96(\mathrm{t}$, $J=7.3 \mathrm{~Hz}, 1 \mathrm{H}), 6.78(\mathrm{~d}, J=7.8 \mathrm{~Hz}, 1 \mathrm{H}), 6.33(\mathrm{~s}, 1 \mathrm{H}), 4.37(\mathrm{q}, J=7.1 \mathrm{~Hz}, 2 \mathrm{H}), 4.23-4.04(\mathrm{~m}, 2 \mathrm{H}), 3.75-3.55(\mathrm{~m}$, 2H), 1.79-1.69 (m, 2H), $1.33(\mathrm{t}, J=7.2 \mathrm{~Hz}, 3 \mathrm{H}), 1.23(\mathrm{~s}, 9 \mathrm{H}), 1.14(\mathrm{~s}, 3 \mathrm{H}), 1.01(\mathrm{t}, J=7.4 \mathrm{~Hz}, 3 \mathrm{H}) ;{ }^{13} \mathrm{C}$ NMR $\left(101 \mathrm{MHz}, \mathrm{CDCl}_{3}\right)$ ס: 173.0, 165.1, 163.7, 155.6 (q, $\left.J=39.4 \mathrm{~Hz}\right), 153.4,145.2,129.7,126.8,125.5,122.2$, 118.5 (q, $J=275.6 \mathrm{~Hz}), 107.9,80.2,63.5,63.2,42.3,28.1,20.6,13.8,13.6,11.6 ;{ }^{19} \mathrm{~F} \mathrm{NMR}\left(376 \mathrm{MHz}, \mathrm{CDCl}_{3}\right) \delta$ : -72.25. HRMS (ESI-TOF): $\mathrm{m} / \mathrm{z}[\mathrm{M}+\mathrm{H}]^{+}$calcd. for $\left[\mathrm{C}_{25} \mathrm{H}_{33} \mathrm{~F}_{3} \mathrm{~N}_{3} \mathrm{O}_{7}\right]^{+}: 544.2278$, found: 544.2285 ; HPLC analysis: The enantiomeric excess was determined by HPLC with a Chiralpak IA column (hexane $/ \mathrm{i}-\mathrm{PrOH}=95 / 5,1.0$ $\left.\mathrm{mL} \cdot \mathrm{min}^{-1}, \lambda=254 \mathrm{~nm}\right): t_{\text {major }}=5.0 \mathrm{~min}, t_{\text {minor }}=5.7 \mathrm{~min}$.

(R,E)-diethyl 2-(1-benzyl-3-((tert-butoxycarbonyl)amino)-2-oxoindolin-3-yl)-2-((2,2,2-trifluoroethylidene)amino) malonate (8I): petroleum ether/ ethyl acetate $=10 / 1$

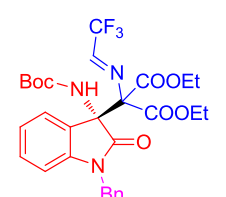

colourless oil, $58.0 \mathrm{mg}, 98 \%$ yield, $>20: 1 \mathrm{dr},>99 \%$ ee, $[a]_{D}^{20}=+45.38(\mathrm{c}=0.42, \mathrm{MeOH})$.

${ }^{1} \mathrm{H}$ NMR $\left(400 \mathrm{MHz}, \mathrm{CDCl}_{3}\right) \delta: 7.69(\mathrm{~d}, J=2.8 \mathrm{~Hz}, 1 \mathrm{H}), 7.52(\mathrm{~d}, J=7.3 \mathrm{~Hz}, 1 \mathrm{H}), 7.46(\mathrm{~d}, J=7.2 \mathrm{~Hz}, 2 \mathrm{H}), 7.30$ (t, $J=7.3 \mathrm{~Hz}, 2 \mathrm{H}), 7.27-7.22(\mathrm{~m}, 1 \mathrm{H}), 7.16(\mathrm{td}, J=7.7,1.1 \mathrm{~Hz}, 1 \mathrm{H}), 6.94(\mathrm{t}, J=7.6 \mathrm{~Hz}, 1 \mathrm{H}), 6.66(\mathrm{~d}, J=7.8 \mathrm{~Hz}$, $1 \mathrm{H}), 6.38(\mathrm{~s}, 1 \mathrm{H}), 4.98(\mathrm{~d}, J=15.6 \mathrm{~Hz}, 1 \mathrm{H}), 4.84(\mathrm{~d}, J=15.7 \mathrm{~Hz}, 1 \mathrm{H}), 4.45-4.35(\mathrm{~m}, 2 \mathrm{H}), 4.14-3.89(\mathrm{~m}, 2 \mathrm{H})$, $1.34(\mathrm{t}, J=7.1 \mathrm{~Hz}, 3 \mathrm{H}), 1.25(\mathrm{~s}, 9 \mathrm{H}), 1.04(\mathrm{~s}, 3 \mathrm{H}) ;{ }^{13} \mathrm{C} \mathrm{NMR}\left(101 \mathrm{MHz}, \mathrm{CDCl}_{3}\right) \delta: 173.5,165.3,163.8,156.0$ (q, $J=39.5 \mathrm{~Hz}$ ), 153.6, 145.0, 135.8, 129.8, 128.7, 127.9, 127.6, 126.8, 125.5, 122.6, 118.6 (q, $J=275.7 \mathrm{~Hz})$, 108.8, 80.4, 63.7, 63.4, 44.8, 28.2, 13.9, 13.6; ${ }^{19} \mathrm{~F}$ NMR (376 MHz, CDCl $)$ $):-72.09$. HRMS (ESI-TOF): $\mathrm{m} / \mathrm{z}$ $[\mathrm{M}+\mathrm{H}]^{+}$calcd. for $\left[\mathrm{C}_{29} \mathrm{H}_{33} \mathrm{~F}_{3} \mathrm{~N}_{3} \mathrm{O}_{7}\right]^{+}:$592.2265, found: 592.2280; HPLC analysis: The enantiomeric excess was determined by HPLC with a Chiralpak IA column (hexane $/ \mathrm{i}-\mathrm{PrOH}=95 / 5,1.0 \mathrm{~mL} \cdot \mathrm{min}^{-1}, \lambda=254 \mathrm{~nm}$ ): $t_{\text {major }}=7.6$ $\min , t_{\operatorname{minor}}=30.6 \mathrm{~min}$.

(R,E)-diethyl 2-(3-((tert-butoxycarbonyl)amino)-2-oxo-1-phenylindolin-3-yl)-2-((2,2,2-trifluoroethylidene)amino) malonate $(\mathbf{8 m})$ : petroleum ether/ ethyl acetate $=10 / 1$

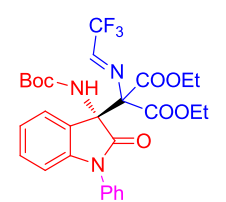

colourless oil, $53.1 \mathrm{mg}, 92 \%$ yield, $>20: 1 \mathrm{dr},>99 \%$ ee, $[a]_{D}^{20}=+42.88(\mathrm{c}=0.35, \mathrm{MeOH})$.

${ }^{1} \mathrm{H}$ NMR $\left(400 \mathrm{MHz}, \mathrm{CDCl}_{3}\right) \delta: 7.77(\mathrm{~d}, J=2.9 \mathrm{~Hz}, 1 \mathrm{H}), 7.57(\mathrm{~d}, J=7.4 \mathrm{~Hz}, 1 \mathrm{H}), 7.54-7.47(\mathrm{~m}, 4 \mathrm{H}), 7.41-7.35(\mathrm{~m}$, $1 \mathrm{H}), 7.21(\mathrm{t}, J=7.8 \mathrm{~Hz}, 1 \mathrm{H}), 7.01(\mathrm{t}, J=7.6 \mathrm{~Hz}, 1 \mathrm{H}), 6.77(\mathrm{~d}, J=7.8 \mathrm{~Hz}, 1 \mathrm{H}), 6.43(\mathrm{~s}, 1 \mathrm{H}), 4.44-4.32(\mathrm{~m}, 2 \mathrm{H})$, 4.28-4.09 (m, 2H), 1.32 (t, $J=7.1 \mathrm{~Hz}, 3 \mathrm{H}), 1.28(\mathrm{~s}, 9 \mathrm{H}), 1.14(\mathrm{~s}, 3 \mathrm{H}) ;{ }^{13} \mathrm{C} \mathrm{NMR}\left(101 \mathrm{MHz}, \mathrm{CDCl}_{3}\right) \delta: 172.7$, 
165.2, 163.6, 156.2 (q, J = 39.5 Hz), 153.8, 145.8, 134.8, 129.7, 129.6, 128.0, 126.7, 125.7, 123.0, 118.7 (q, J = $275.6 \mathrm{~Hz}$ ), 109.1, 80.5, 63.8, 63.6, 28.3, 13.9, 13.7; ${ }^{19} \mathrm{~F}$ NMR (376 MHz, $\mathrm{CDCl}_{3}$ ) ס: -72.18. HRMS (ESI-TOF): $\mathrm{m} / \mathrm{z}[\mathrm{M}+\mathrm{H}]^{+}$calcd. for $\left[\mathrm{C}_{28} \mathrm{H}_{31} \mathrm{~F}_{3} \mathrm{~N}_{3} \mathrm{O}_{7}\right]^{+}: 578.2257$, found: 578.2260; HPLC analysis: The enantiomeric excess was determined by HPLC with a Chiralpak IA column (hexane $/ \mathrm{i}-\mathrm{PrOH}=95 / 5,0.8 \mathrm{~mL} \cdot \mathrm{min}^{-1}, \lambda=254 \mathrm{~nm}$ ): $t_{\text {major }}=$ $6.3 \mathrm{~min}, t_{\mathrm{minor}}=7.0 \mathrm{~min}$.

\section{g. Three Components Reaction}
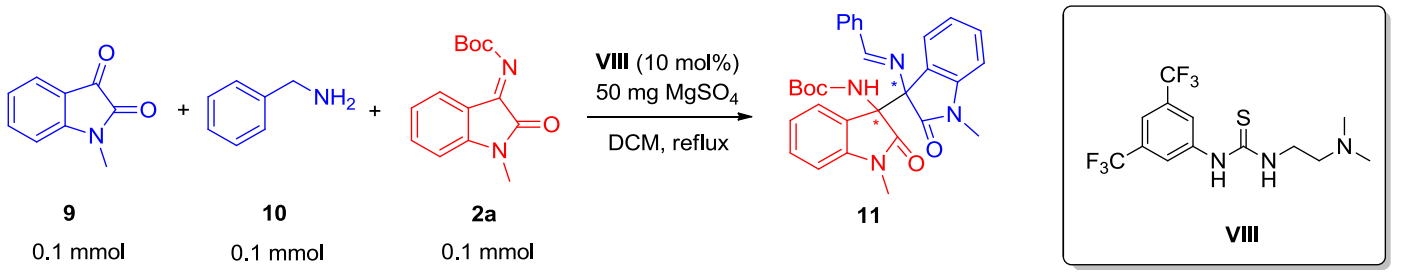

To a solution of isatin-derived $N$-Boc ketimine $2(0.10 \mathrm{mmol}, 1.0$ equiv. $)$ and VIII $(0.01 \mathrm{mmol}, 10 \mathrm{~mol} \%)$ in DCM (1 mL) was added isatin 9 ( $0.10 \mathrm{mmol}, 1.0$ equiv.) and benzyl amine 10 (0.10 mmol, 1.0 equiv, ). Then 50 $\mathrm{mg} \mathrm{MgSO}_{4}$ was added. The mixture was refluxed in oil bath until the reaction was completed (monitored by TLC analysis). The crude product was purified directly by flash column chromatography on silica gel (petroleum ether/ ethyl acetate $=5: 1 \sim 2: 1$ ) to give the desired racemic product 11.
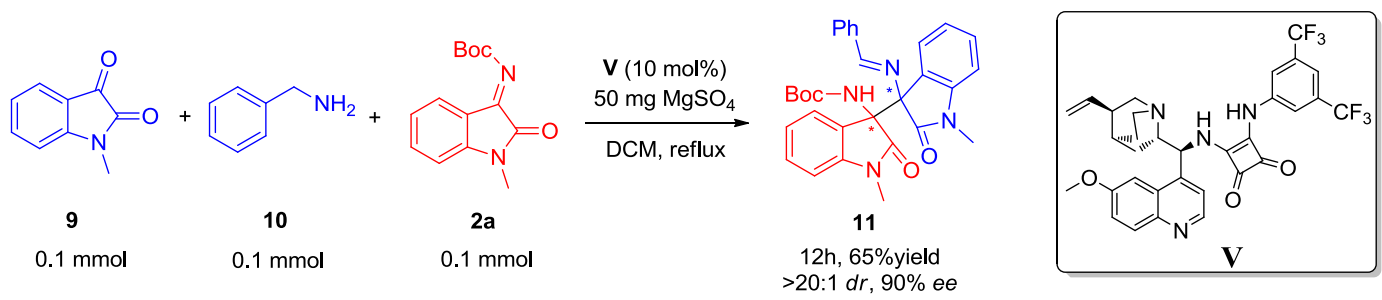

To a solution of isatin-derived $\mathrm{N}$-Boc ketimine $2(0.10 \mathrm{mmol}, 1.0$ equiv. $)$ and $\mathbf{V}(0.01 \mathrm{mmol}, 10 \mathrm{~mol} \%)$ in $\mathrm{DCM}(1 \mathrm{~mL})$ was added isatin 9 ( $0.10 \mathrm{mmol}, 1.0$ equiv.) and benzyl amine 10 (0.10 mmol, 1.0 equiv.). Then 50 $\mathrm{mg} \mathrm{MgSO}_{4}$ was added. The mixture was refluxed in oil bath until the reaction was completed (monitored by TLC analysis). The crude product was purified directly by flash column chromatography on silica gel (petroleum ether/ ethyl acetate $=5: 1 \sim 2: 1$ ) to give the desired chiral product 11 as a colourless oil.

(E)-tert-butyl (3'-(benzylideneamino)-1,1'-dimethyl-2,2'-dioxo-[3,3'-biindolin]-3-yl)carbamate (11): petroleum ether/ ethyl acetate $=5 / 1 \sim 2 / 1$

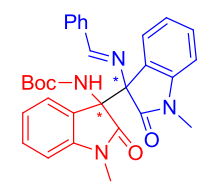

colourless oil, $33.2 \mathrm{mg}, 65 \%$ yield, $>20: 1 \mathrm{dr}, 90 \%$ ee, $[a]_{D}^{20}=+49.20(\mathrm{c}=0.23, \mathrm{MeOH})$.

${ }^{1} \mathbf{H}$ NMR $\left(400 \mathrm{MHz}, \mathrm{CDCl}_{3}\right) \delta: 7.97(\mathrm{~s}, 1 \mathrm{H}), 7.82(\mathrm{~d}, J=7.0 \mathrm{~Hz}, 2 \mathrm{H}), 7.65-7.39(\mathrm{~m}, 5 \mathrm{H}), 7.35(\mathrm{t}, J=7.7 \mathrm{~Hz}, 1 \mathrm{H})$, 7.27-7.21 (m, 1H), 7.05 (t, $J=7.4 \mathrm{~Hz}, 1 \mathrm{H}), 6.82(\mathrm{~d}, J=7.8 \mathrm{~Hz}, 1 \mathrm{H}), 6.68(\mathrm{t}, J=6.2 \mathrm{~Hz}, 2 \mathrm{H}), 5.63(\mathrm{~s}, 1 \mathrm{H}), 3.27(\mathrm{~s}$, 3H), $2.74(\mathrm{~s}, 3 \mathrm{H}), 1.25$ (s, 9H); ${ }^{13} \mathrm{C}$ NMR $\left(101 \mathrm{MHz}, \mathrm{CDCl}_{3}\right) \delta: 173.5,172.9,163.1,154.3,144.8,144.2,135.6$, 131.8, 130.2, 129.5, 129.0, 128.8, 125.7, 125.3, 124.1, 122.3, 121.9, 108.7, 107.7, 79.9, 72.8, 67.6, 28.3, 26.5, 25.9. HRMS (ESI-TOF): $\mathrm{m} / z[\mathrm{M}+\mathrm{Na}]^{+}$calcd. for $\left[\mathrm{C}_{30} \mathrm{H}_{30} \mathrm{~N}_{4} \mathrm{NaO}_{4}\right]^{+}: 533.2021$, found: 533.2020; HPLC analysis: The enantiomeric excess was determined by HPLC with a Chiralpak IB column (hexane/i-PrOH $=75 / 25,0.8$ 
$\left.\mathrm{mL} \cdot \mathrm{min}^{-1}, \lambda=280 \mathrm{~nm}\right): t_{\text {major }}=8.3 \mathrm{~min}, t_{\text {minor }}=9.4 \mathrm{~min}$.

\section{h. The Gram Scale Reactions of $3 a$ and $8 a$}<smiles>CN1C(=O)/C(=N/CC(F)(F)F)c2ccccc21</smiles>

1a

$3.0 \mathrm{mmol}$

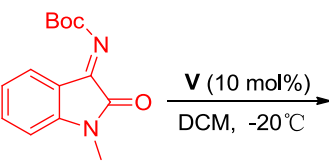

2a

$3.0 \mathrm{mmo}$

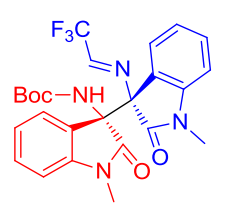

$3 \mathbf{a}$

$1.28 \mathrm{~g}, 85 \%$ yield $>20: 1 d r, 94 \%$ ee

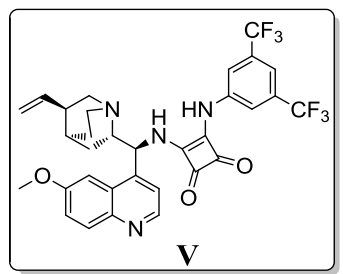

V

To a solution of $\mathrm{N}$-2,2,2-trifluoroethylisatin ketimine $1 \mathrm{a}$ (3.00 mmol, 1.0 equiv.), $1.20 \mathrm{~g} 4 \AA$ molecular sieves, and catalyst $\mathbf{V}(0.30 \mathrm{mmol}, 10 \mathrm{~mol} \%)$ in DCM $(20 \mathrm{~mL})$ was added isatin-derived $\mathrm{N}$-Boc ketimine $2 \mathbf{a}(3.00 \mathrm{mmol}$, 1.0 equiv.). The mixture was stirred at $-20{ }^{\circ} \mathrm{C}$ until the reaction was completed (monitored by TLC analysis). The crude product was purified directly by flash column chromatography on silica gel (petroleum ether/ ethyl acetate $=10: 1 \sim 5: 1)$ to give the desired chiral product $3 \mathrm{a}$ as a white solid $(1.28 \mathrm{~g}, 85 \%$ yield, $>20: 1 d r, 94 \%$ ee $)$.

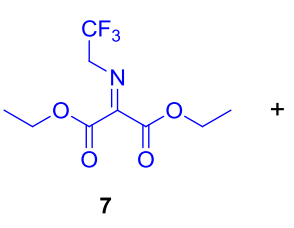

$4.5 \mathrm{mmol}$<smiles>CN1C(=O)/C(=N/C(C)(C)C)c2ccccc21</smiles>

2a

$3.0 \mathrm{mmol}$

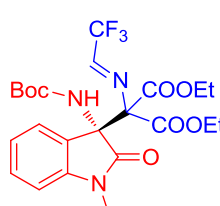

$8 \mathbf{a}$

$1.33 \mathrm{~g}, 86 \%$ yield

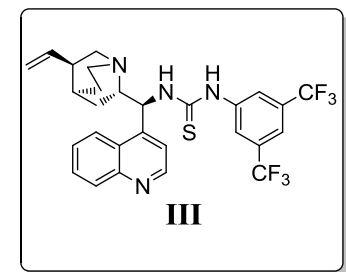

III

To a solution of isatin-derived $\mathrm{N}$-Boc ketimine 2a (3.00 mmol, 1.0 equiv.) and catalyst III $(0.30 \mathrm{mmol}, 10$ mol\%) in DCE (20 mL) was added diethyl 2-[(2,2,2-trifluoroethyl)-imino]malonate 7 (4.50 mmol, 1.5 equiv.). The mixture was stirred at $-20^{\circ} \mathrm{C}$ until the reaction was completed (monitored by TLC analysis). The crude product was purified directly by flash column chromatography on silica gel (petroleum ether/ ethyl acetate $=10: 1 \sim 5: 1$ ) to give the desired chiral product $8 \mathrm{a}$ as a white foam $(1.33 \mathrm{~g}, 86 \%$ yield, $99 \%$ ee). 


\section{Proposed Reaction Mechanism}

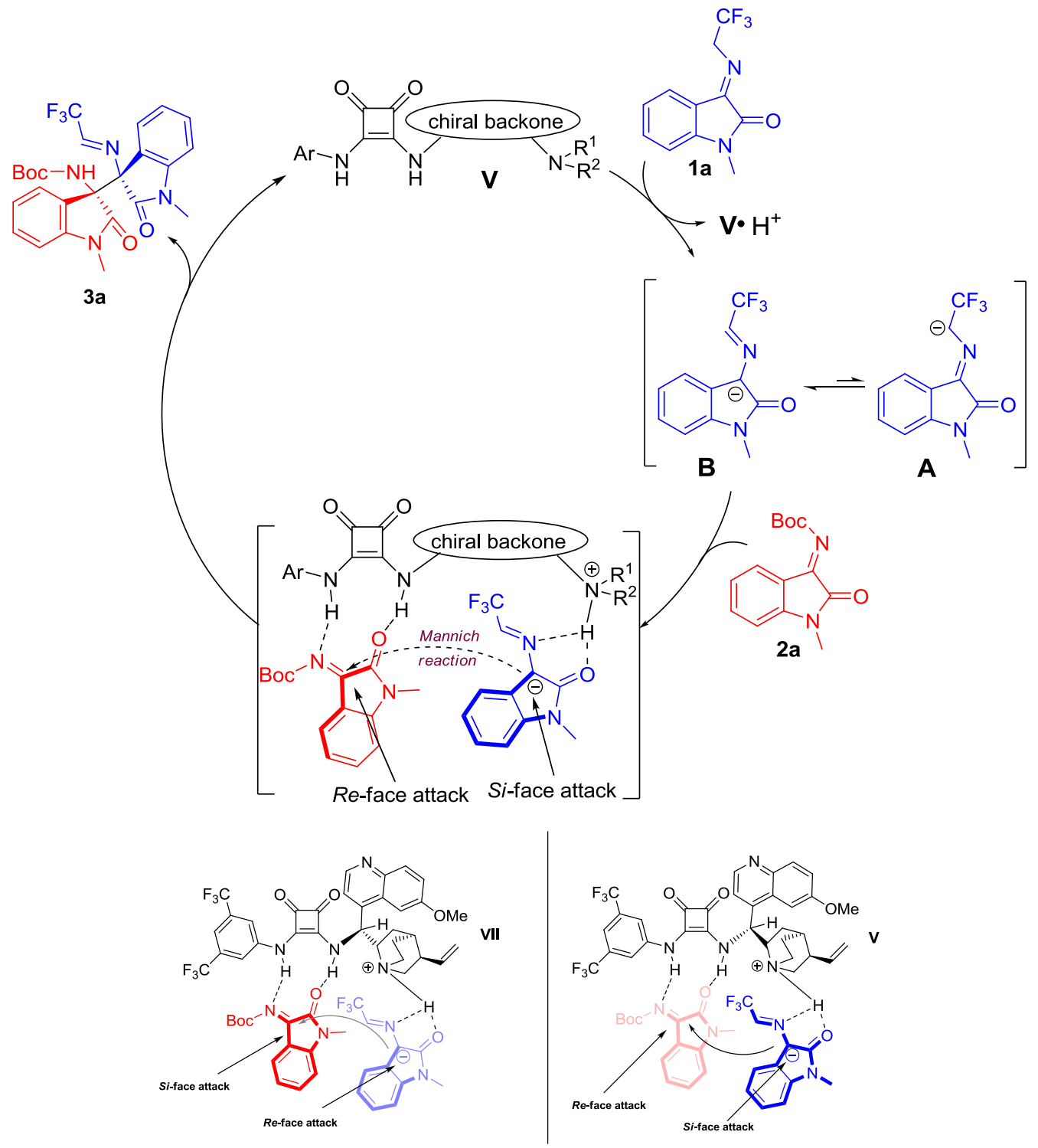

Figure S1. Proposed reaction mechanism

According to the absolute configuration of $\mathbf{3 d}$ and literature reports, ${ }^{2}$ we proposed a possible reaction mechanism for the catalytic asymmetric umpolung cross-Mannich reaction as shown in Figure S1. Catalyst V initially promoted the formation of resonance forms $\mathbf{A}$ and $\mathbf{B}$ through the deprotonation of the precursor $\mathbf{1 a}$. Both resonance forms and $\mathbf{2} \mathbf{a}$ are simultaneously activated via hydrogen-bonding interactions. The squaramide moiety of catalyst $\mathbf{V}$ formed two hydrogen bonds corresponding with the carbonyl and ketimine groups of the isatin-derived $\mathrm{N}$-Boc ketimine $\mathbf{2 a}$. The tertiary amine moiety deprotonated and activated the $\mathrm{N}$-2,2,2-trifluoroethylisatin ketimine via double hydrogen bonds. In this asymmetry umpolung cross-Mannich reaction step, the $\mathrm{C}_{3}$-position (Si-face) of activated $\mathrm{N}$-2,2,2-trifluoroethylisatin ketamine attacks the isatin-derived $\mathrm{N}$-Boc ketimine 2a (Re-face), resulting in the desired 3,3'-diamines bisoxindoles 3a with fixed stereochemistry (Figure S1). 


\section{Data for X-Ray Crystal Structure of $3 d$ and $8 d$}

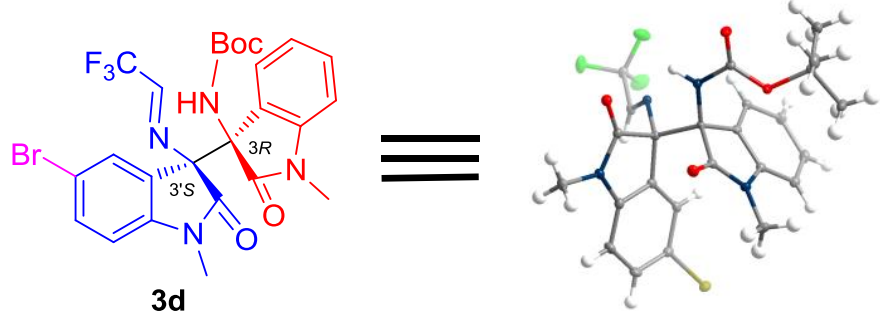

Table S1 Crystal data and structure refinement for $\mathbf{3 d}$ (ellipsoids: $30 \%$ probability).

\begin{tabular}{|c|c|}
\hline Empirical formula & $\mathrm{C}_{25} \mathrm{H}_{24} \mathrm{~N}_{4} \mathrm{O}_{4} \mathrm{BrF}_{3}$ \\
\hline Formula weight & 581.39 \\
\hline Temperature/K & $100.00(10)$ \\
\hline Crystal system & orthorhombic \\
\hline Space group & $\mathrm{P} 2{ }_{1} 2_{1} 2_{1}$ \\
\hline $\mathrm{a} / \AA ̊ \AA$ & $9.92570(10)$ \\
\hline$b / \AA$ & $13.16480(10)$ \\
\hline$c / \AA ̊$ & $19.58350(10)$ \\
\hline$\alpha /^{\circ}$ & 90 \\
\hline$\beta /^{\circ}$ & 90 \\
\hline $\mathrm{y} /^{\circ}$ & 90 \\
\hline Volume/ $/ \AA^{3}$ & $2558.97(3)$ \\
\hline Z & 4 \\
\hline$\rho_{\text {calc }} \mathrm{g} / \mathrm{cm}^{3}$ & 1.509 \\
\hline$\mu / \mathrm{mm}^{-1}$ & 2.730 \\
\hline$F(000)$ & 1184.0 \\
\hline Crystal size $/ \mathrm{mm}^{3}$ & $0.3 \times 0.2 \times 0.1$ \\
\hline Radiation & $\operatorname{CuKa}(\lambda=1.54184)$ \\
\hline $2 \Theta$ range for data collection $/^{\circ}$ & 8.092 to 144.254 \\
\hline Index ranges & $-11 \leq h \leq 12,-16 \leq k \leq 12,-19 \leq \mathrm{I} \leq 24$ \\
\hline Reflections collected & 25496 \\
\hline Independent reflections & $5052\left[R_{\text {int }}=0.0414, R_{\text {sigma }}=0.0240\right]$ \\
\hline Data/restraints/parameters & $5052 / 0 / 339$ \\
\hline Goodness-of-fit on $\mathrm{F}^{2}$ & 1.060 \\
\hline Final $R$ indexes $[\mid>=2 \sigma(I)]$ & $\mathrm{R}_{1}=0.0259, \mathrm{wR}_{2}=0.0665$ \\
\hline Final $R$ indexes [all data] & $\mathrm{R}_{1}=0.0265, \mathrm{wR}_{2}=0.0667$ \\
\hline Largest diff. peak/hole / e $\AA^{-3}$ & $0.45 /-0.30$ \\
\hline Flack parameter & $-0.014(6)$ \\
\hline
\end{tabular}




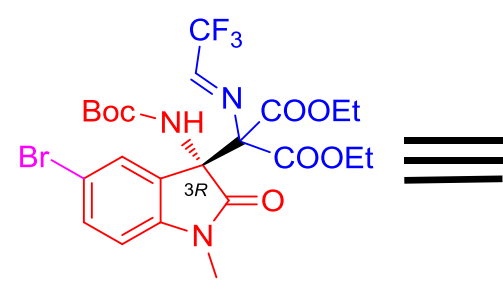

8d

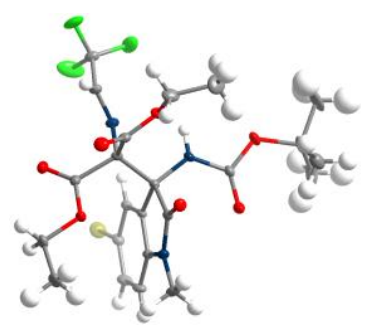

Table S2 Crystal data and structure refinement for $\mathbf{8 d}$ (ellipsoids: $\mathbf{3 0} \%$ probability)

Empirical formula

Formula weight

Temperature/K

Crystal system

Space group

$\mathrm{a} / \mathrm{A}$

$\mathrm{b} / \AA$

$c / \AA ̊ ̊$

$\alpha /^{\circ}$

$\beta /^{\circ}$

$\mathrm{Y} /{ }^{\circ}$

Volume $/ \AA^{3}$

Z

$\rho_{\text {calc }} \mathrm{g} / \mathrm{cm}^{3}$

$\mu / \mathrm{mm}^{-1}$

$F(000)$

Crystal size $/ \mathrm{mm}^{3}$

Radiation

$2 \Theta$ range for data collection/ ${ }^{\circ}$

Index ranges

Reflections collected

Independent reflections

Data/restraints/parameters

Goodness-of-fit on $\mathrm{F}^{2}$

Final $R$ indexes $[\mid>=2 \sigma(I)]$

Final $R$ indexes [all data]

Largest diff. peak/hole/e $\AA^{-3}$

Empirical formula
$\mathrm{C}_{23} \mathrm{H}_{27} \mathrm{BrF}_{3} \mathrm{~N}_{3} \mathrm{O}_{7}$

594.38

100.0

monoclinic

$P 2_{1}$

16.63910(10)

$10.19740(10)$

$16.70830(10)$

90

101.9360(10)

90

2773.70(4)

4

1.423

2.600

1216.0

$0.4 \times 0.3 \times 0.2$

CuKa $(\lambda=1.54178)$

5.406 to 152.906

$-20 \leq \mathrm{h} \leq 20$,

$-12 \leq \mathrm{k} \leq 12$,

$-20 \leq \mathrm{I} \leq 21$

54608

11429 [Rint $=0.0627$,

Rsigma $=0.0345]$

$11429 / 1 / 667$

1.026

$R_{1}=0.0391, w R_{2}=0.1058$

$\mathrm{R}_{1}=0.0397, \mathrm{wR}_{2}=0.1066$

0.33/-0.90

$\mathrm{C}_{23} \mathrm{H}_{27} \mathrm{BrF}_{3} \mathrm{~N}_{3} \mathrm{O}_{7}$

$R_{1}=\Sigma|| F_{0}|-| F_{\mathrm{c}}|| \sum\left|F_{0}\right| \cdot w R_{2}=\left[\sum w\left(F_{0}^{2}-F_{\mathrm{c}}^{2}\right)^{2} / \Sigma w\left(F_{0}^{2}\right)^{2}\right]^{1 / 2}$. 


\section{Copies of NMR Spectra of Compounds}

${ }^{1} \mathrm{H}$ NMR of compound $3 \mathrm{a}\left(400 \mathrm{MHz}\right.$ in $\left.\mathrm{CDCl}_{3}\right)$

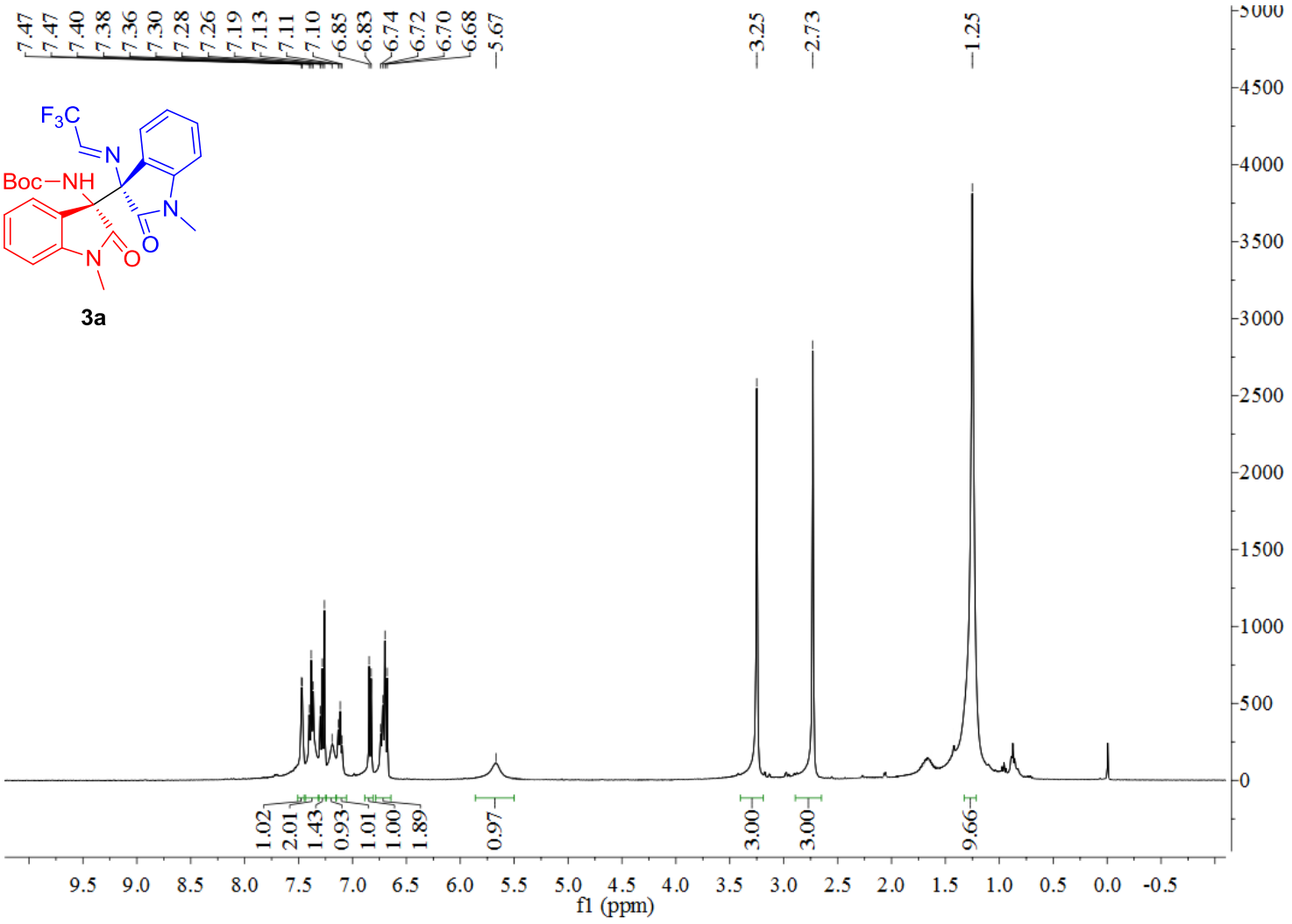

${ }^{13} \mathrm{C}$ NMR of compound $3 \mathrm{a}$ (101 $\mathrm{MHz}$ in $\mathrm{CDCl}_{3}$ )

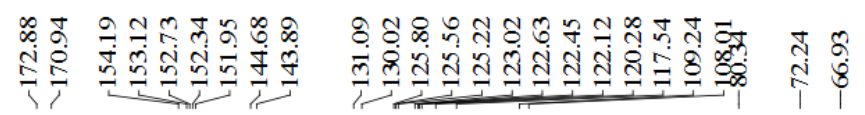

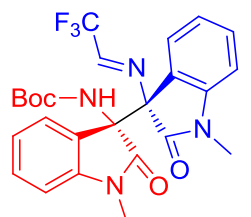

3a

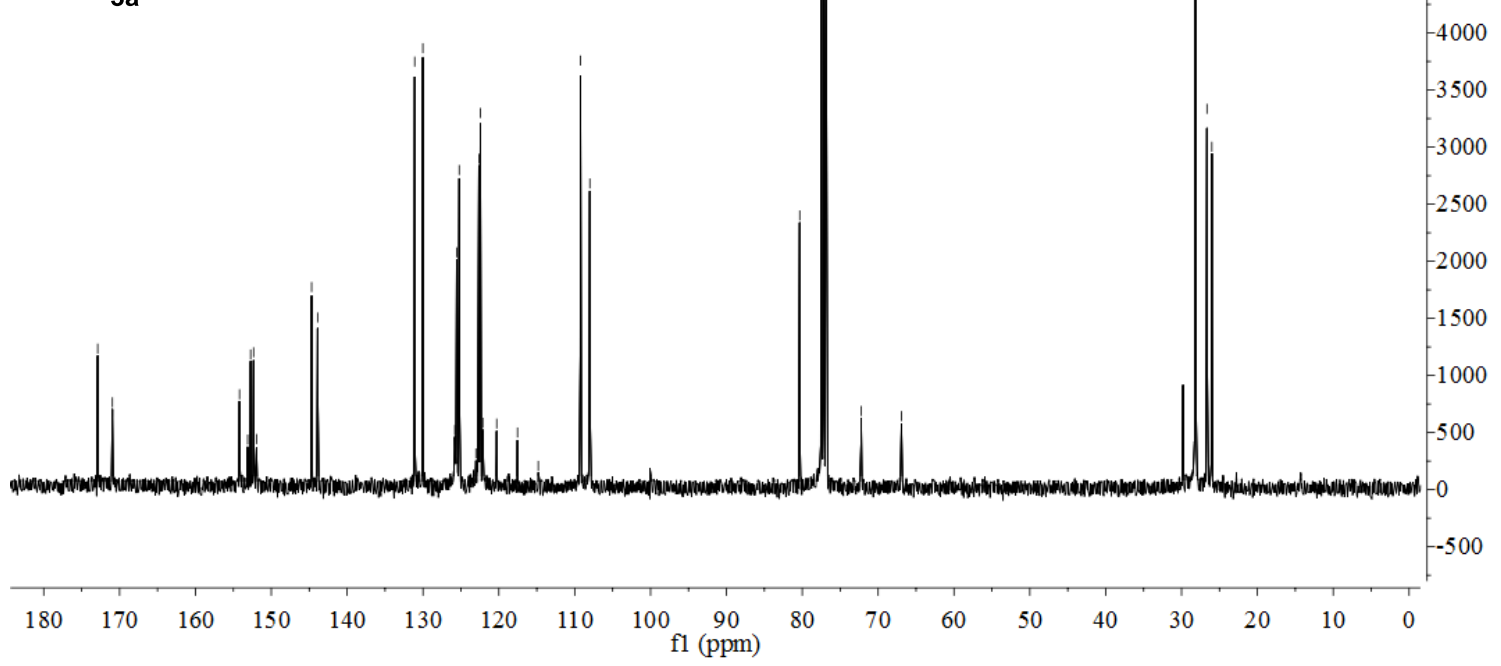


${ }^{19} \mathrm{~F}$ NMR of compound $3 \mathrm{a}\left(376 \mathrm{MHz}\right.$ in $\left.\mathrm{CDCl}_{3}\right)$

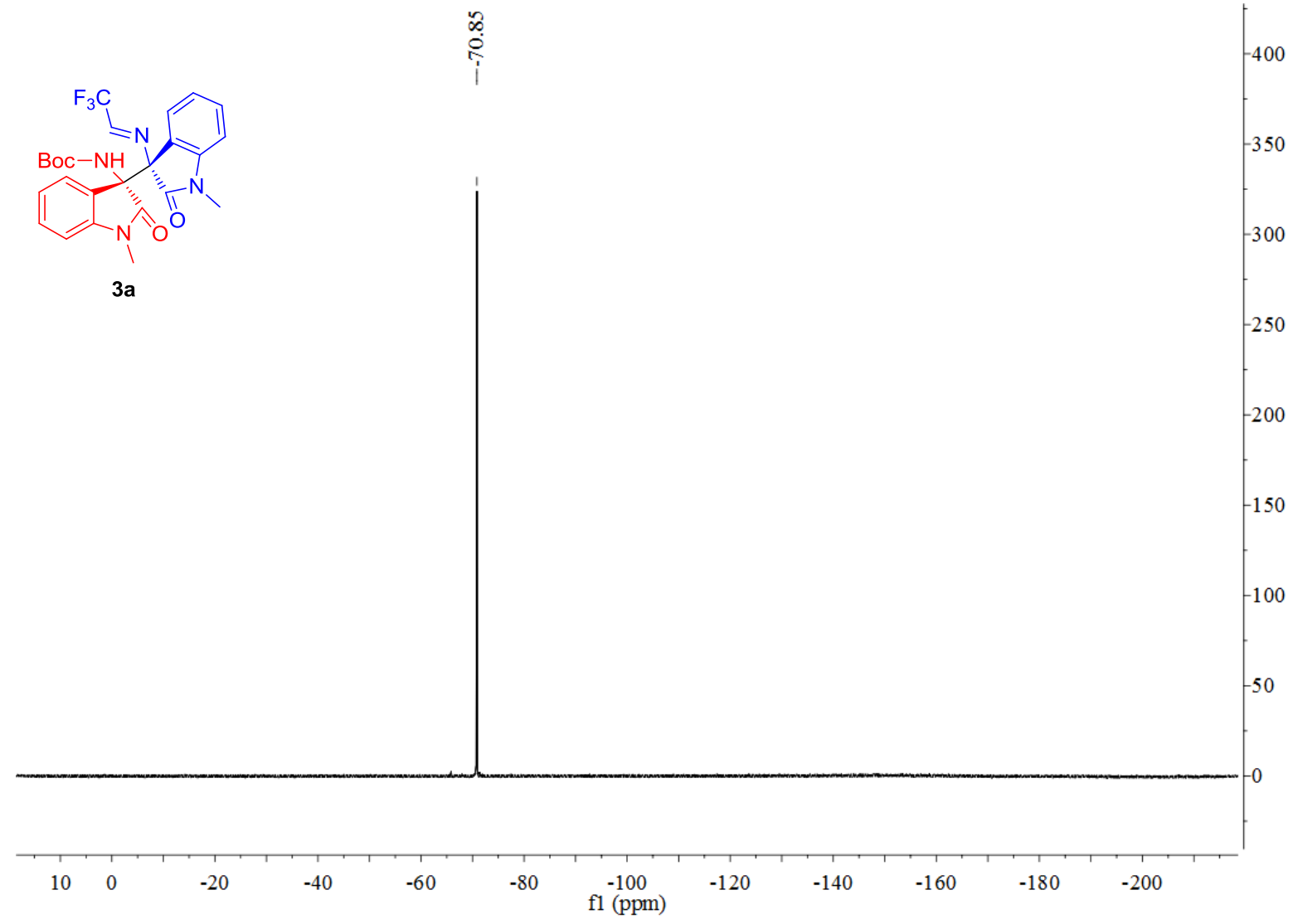

${ }^{1} \mathrm{H}$ NMR of compound $3 \mathrm{~b}\left(400 \mathrm{MHz}\right.$ in $\mathrm{CDCl}_{3}$ )

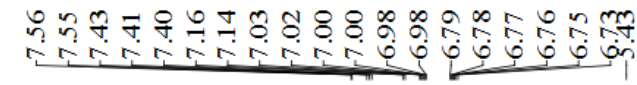

$\stackrel{\vec{i}}{\stackrel{i}{i}}$

$\stackrel{\text { ก }}{7}$

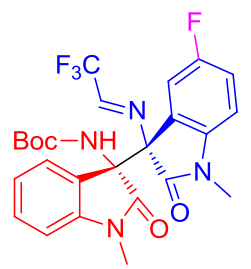

3b

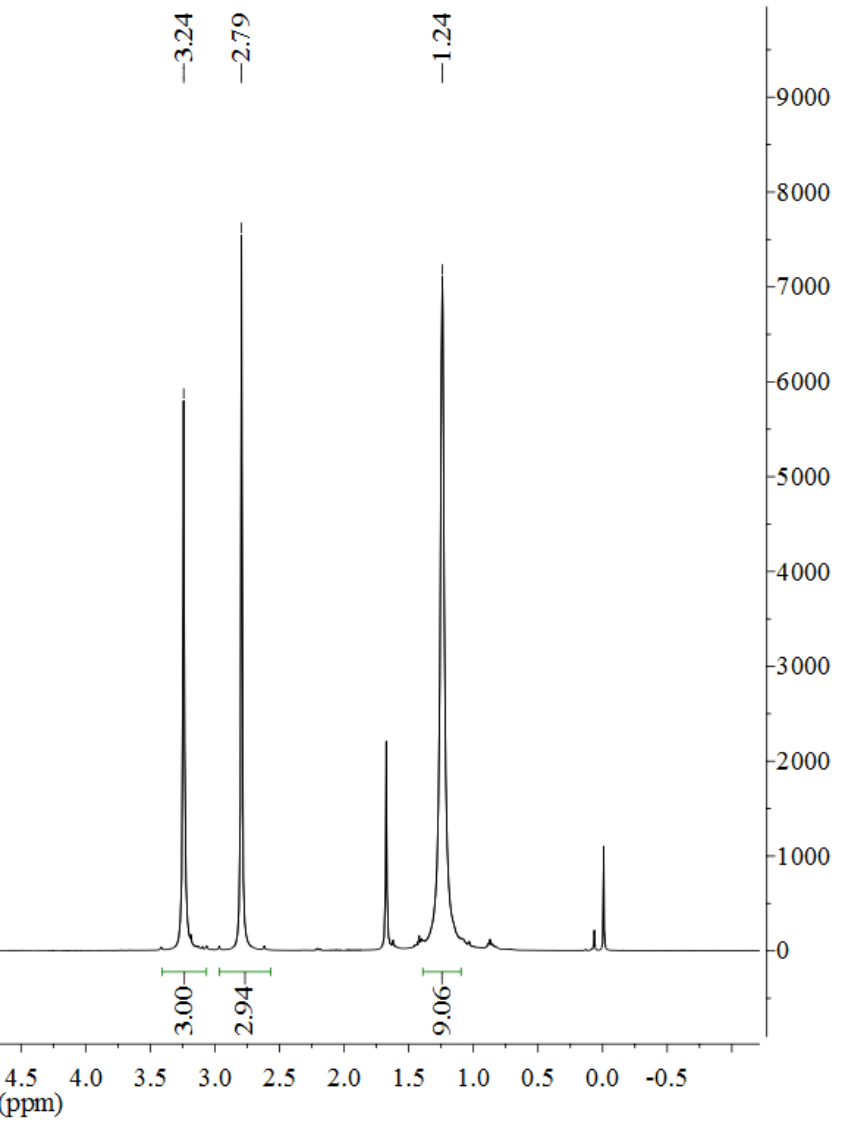


${ }^{13} \mathrm{C}$ NMR of compound $3 \mathrm{~b}\left(101 \mathrm{MHz}\right.$ in $\mathrm{CDCl}_{3}$ )

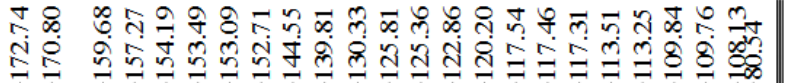

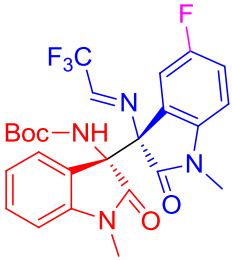

$3 b$

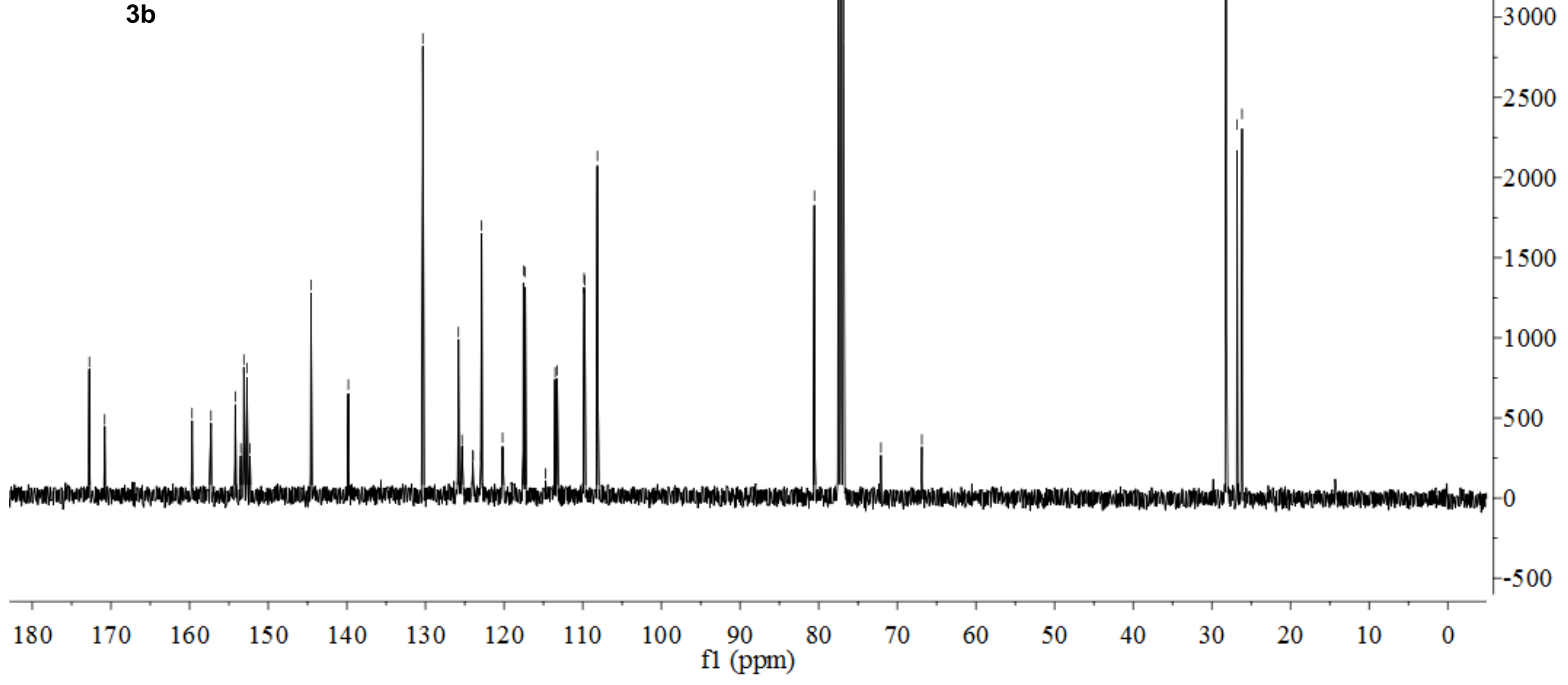

${ }^{19} \mathrm{~F} \mathrm{NMR}$ of compound $3 \mathrm{~b}$ (376 $\mathrm{MHz}$ in $\mathrm{CDCl}_{3}$ )

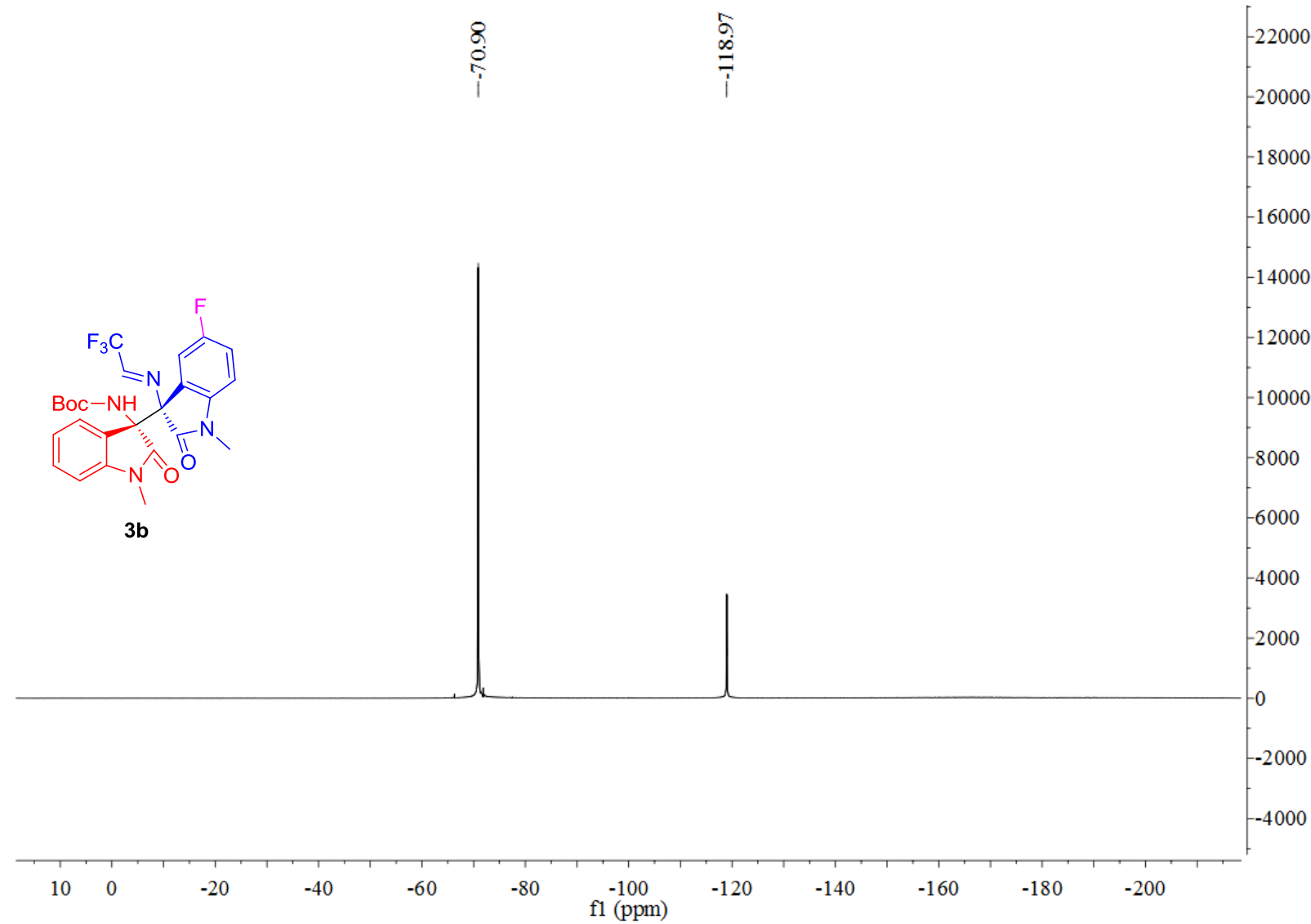


${ }^{1} \mathrm{H}$ NMR of compound $3 \mathrm{c}\left(400 \mathrm{MHz}\right.$ in $\left.\mathrm{CDCl}_{3}\right)$

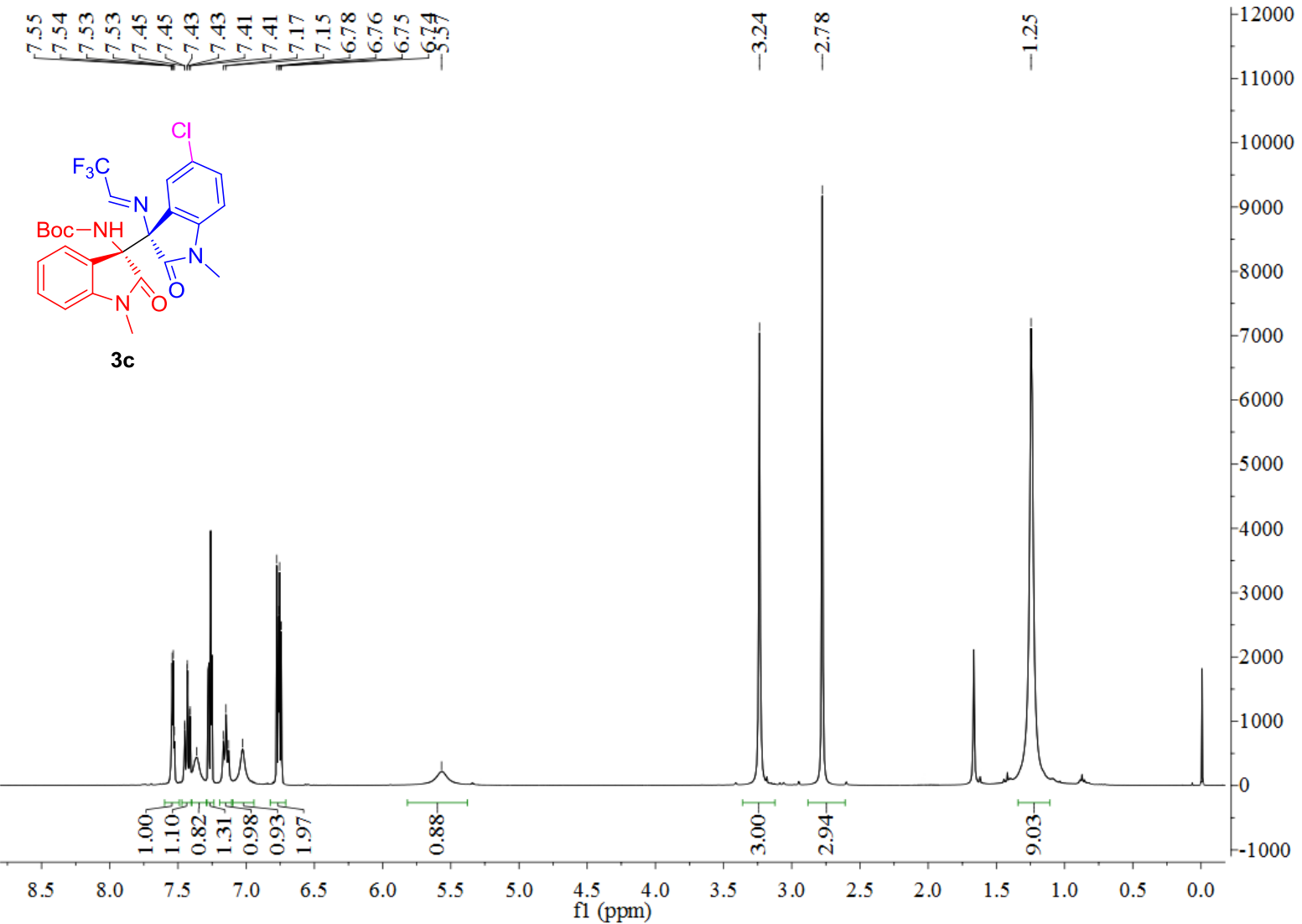

${ }^{13} \mathrm{C}$ NMR of compound 3c (101 $\mathrm{MHz}$ in $\left.\mathrm{CDCl}_{3}\right)$

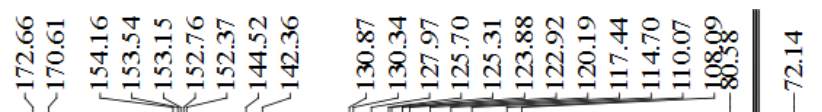

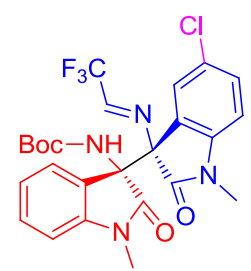

$3 c$

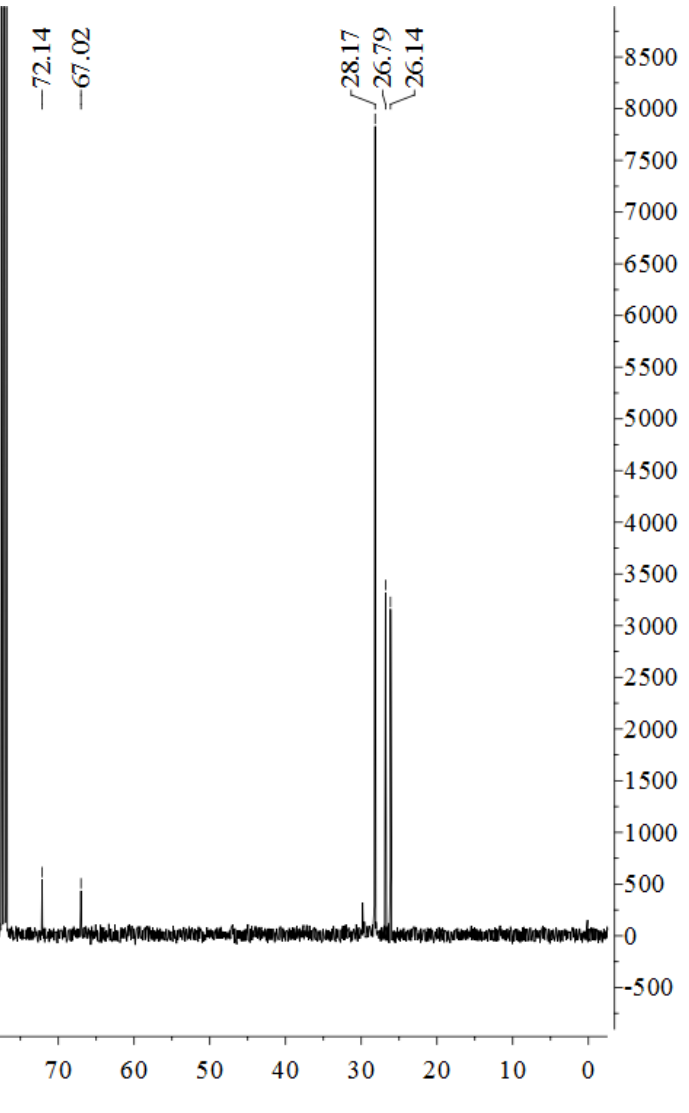


${ }^{19} \mathrm{~F} \mathrm{NMR}$ of compound $3 \mathrm{c}\left(376 \mathrm{MHz}\right.$ in $\left.\mathrm{CDCl}_{3}\right)$

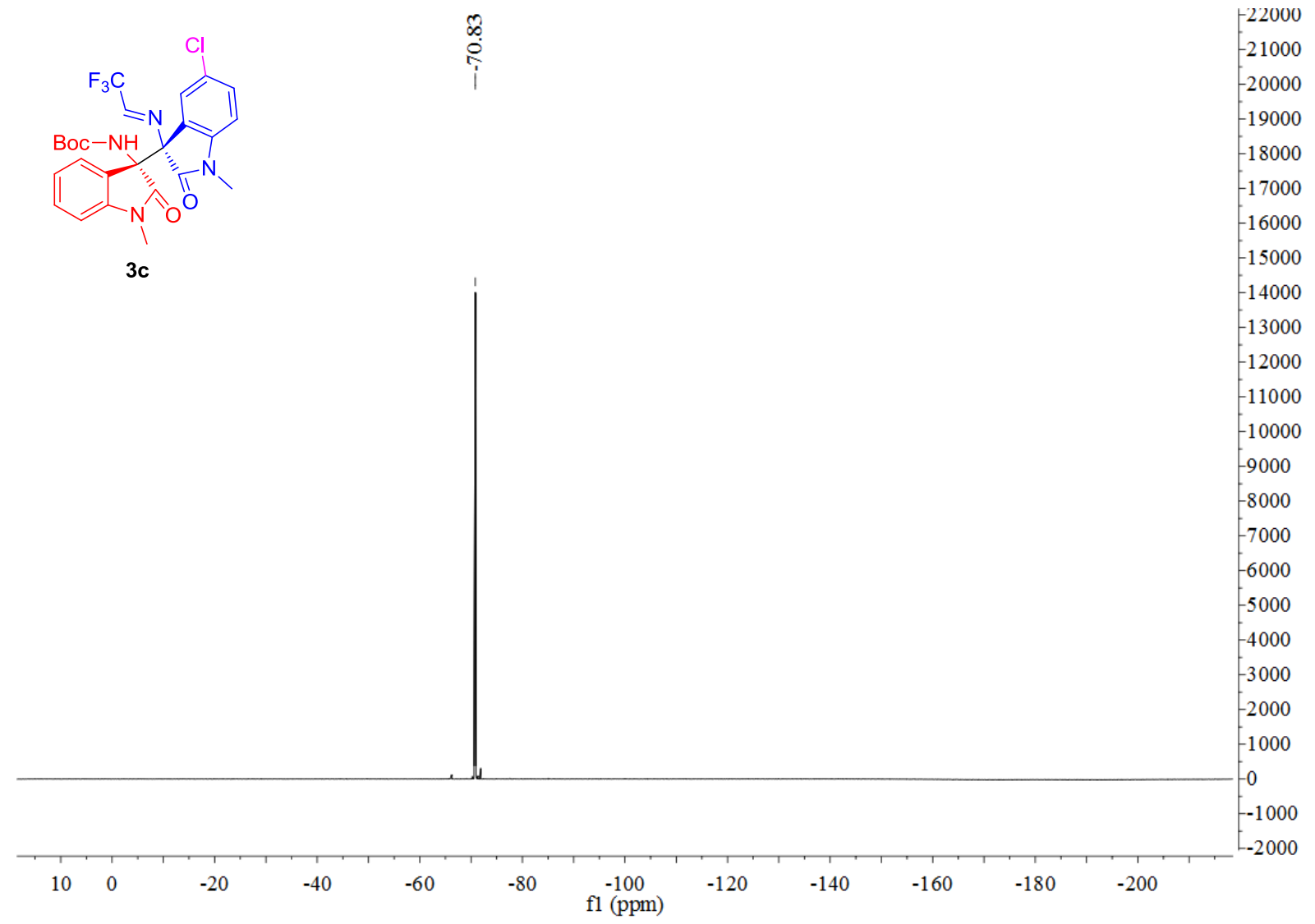

${ }^{1} \mathrm{H}$ NMR of compound $3 \mathrm{~d}\left(400 \mathrm{MHz}\right.$ in $\left.\mathrm{CDCl}_{3}\right)$

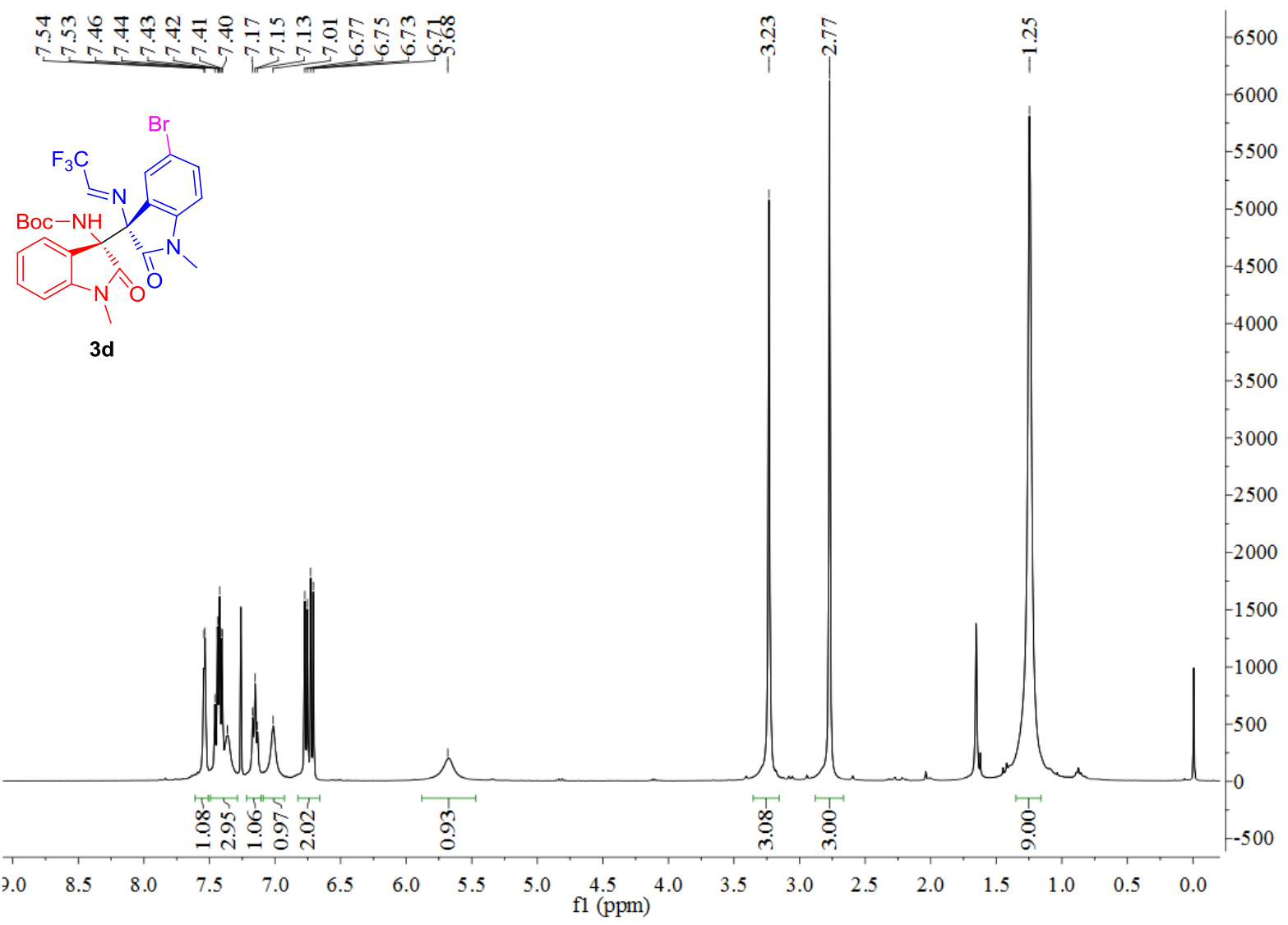


${ }^{13} \mathrm{C}$ NMR of compound $3 \mathrm{~d}\left(101 \mathrm{MHz}\right.$ in $\left.\mathrm{CDCl}_{3}\right)$

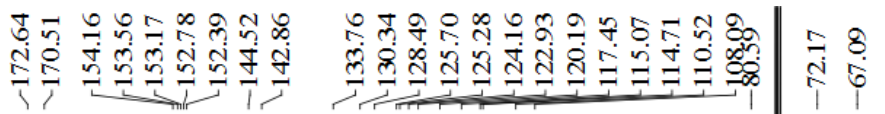

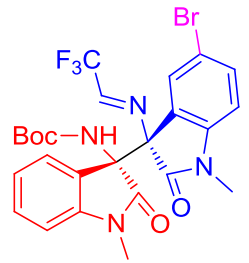

3d

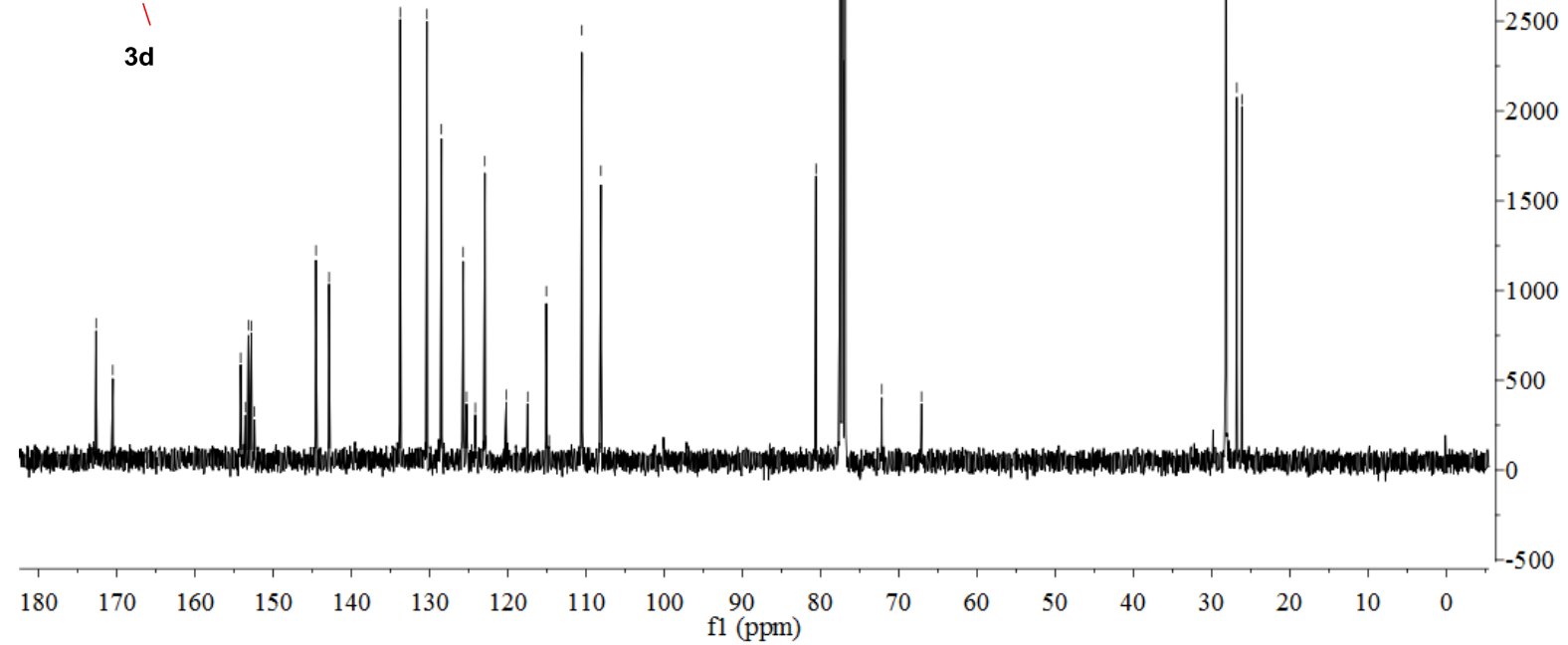

${ }^{19} \mathrm{~F}$ NMR of compound $3 \mathrm{~d}\left(376 \mathrm{MHz}\right.$ in $\left.\mathrm{CDCl}_{3}\right)$

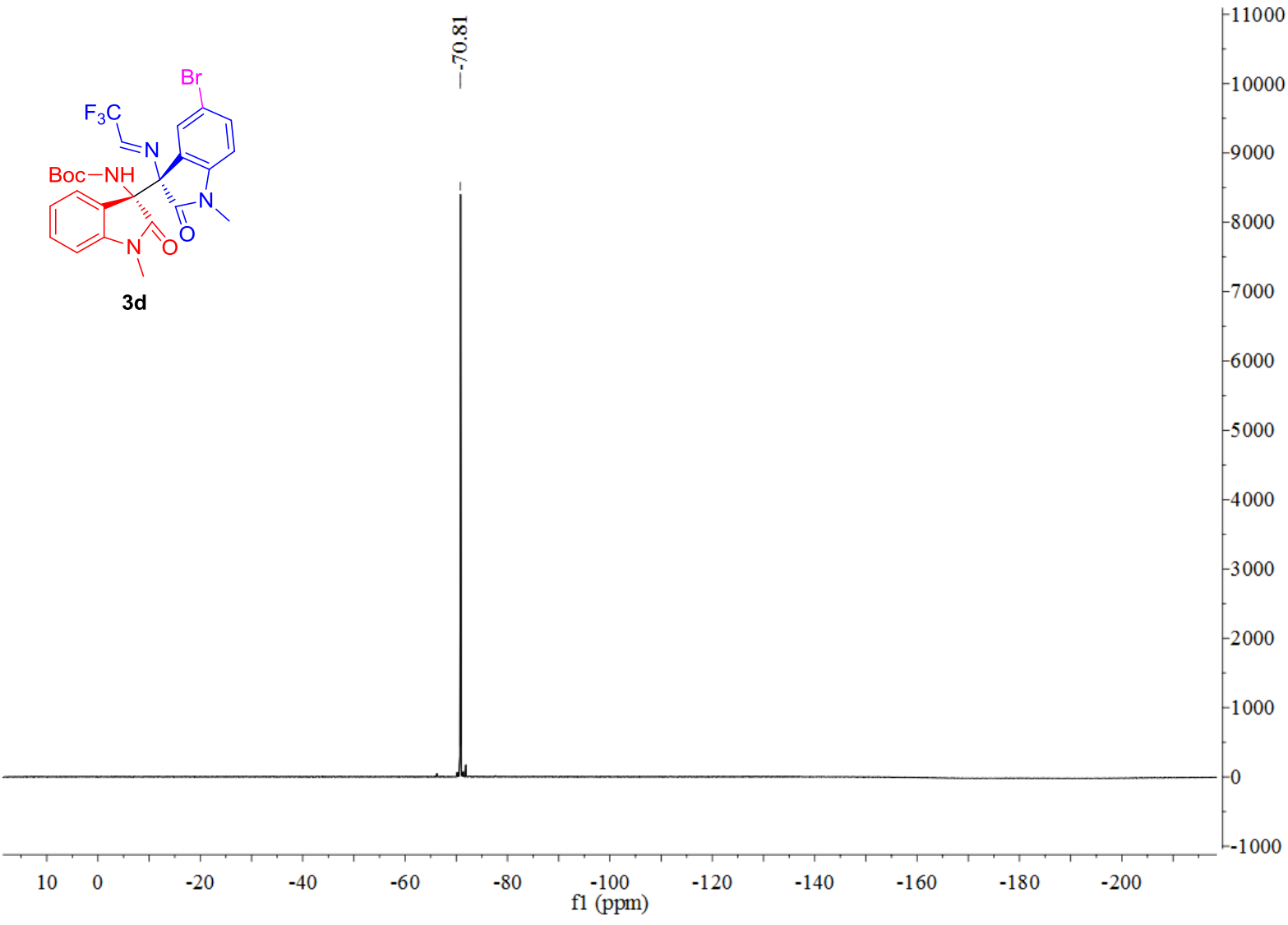


${ }^{1} \mathrm{H}$ NMR of compound $3 \mathrm{e}\left(400 \mathrm{MHz}\right.$ in $\left.\mathrm{CDCl}_{3}\right)$

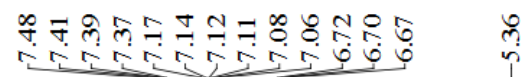

î

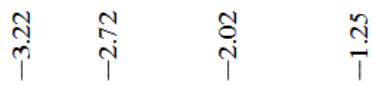

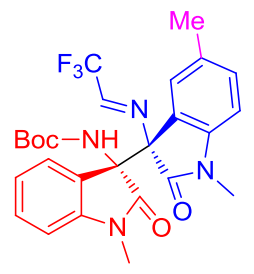

$3 e$

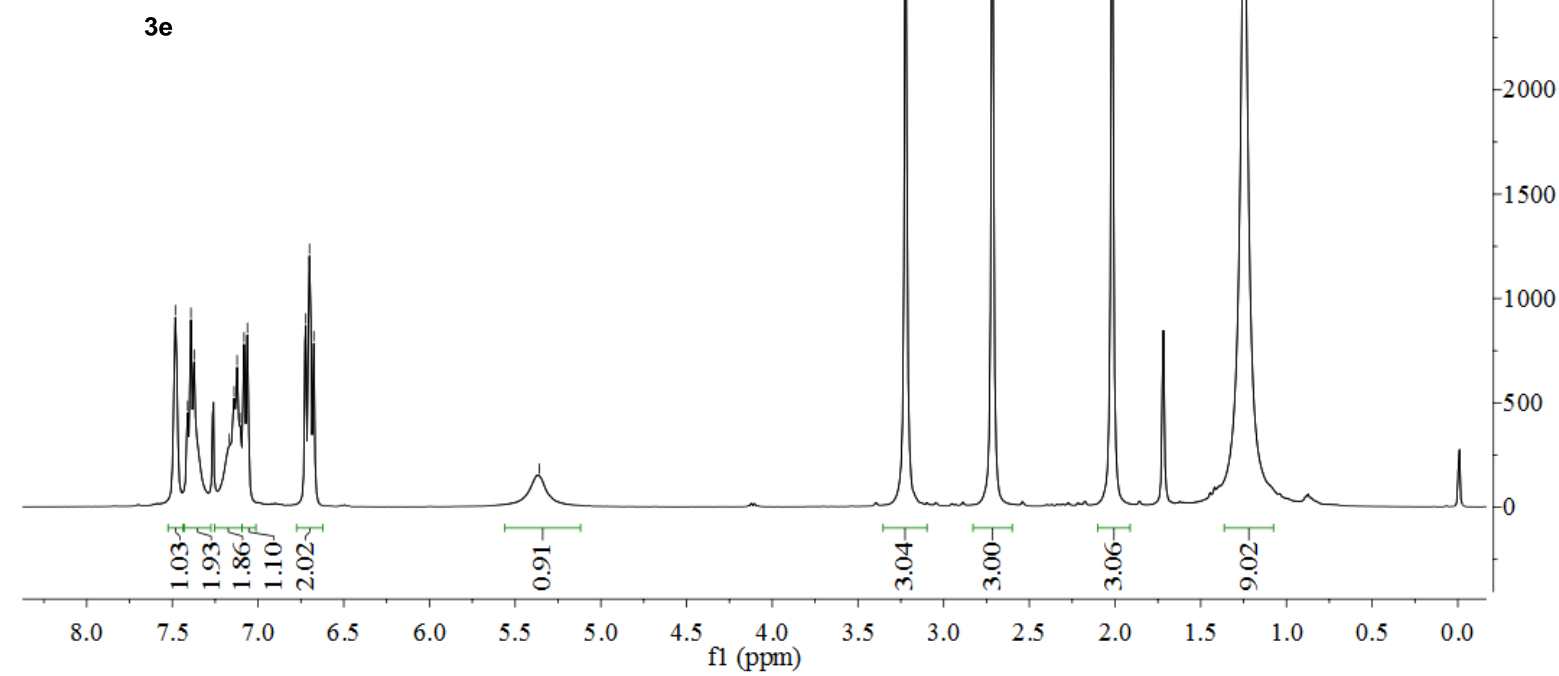

${ }^{13} \mathrm{C} \mathrm{NMR}$ of compound $3 \mathrm{e}\left(126 \mathrm{MHz}\right.$ in $\left.\mathrm{CDCl}_{3}\right)$

×

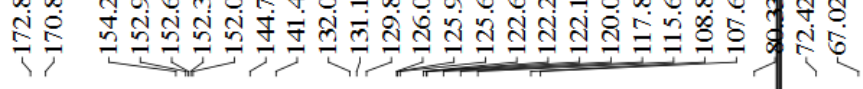

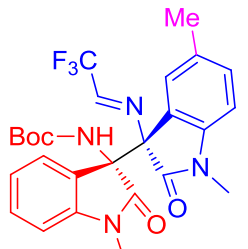

$3 e$

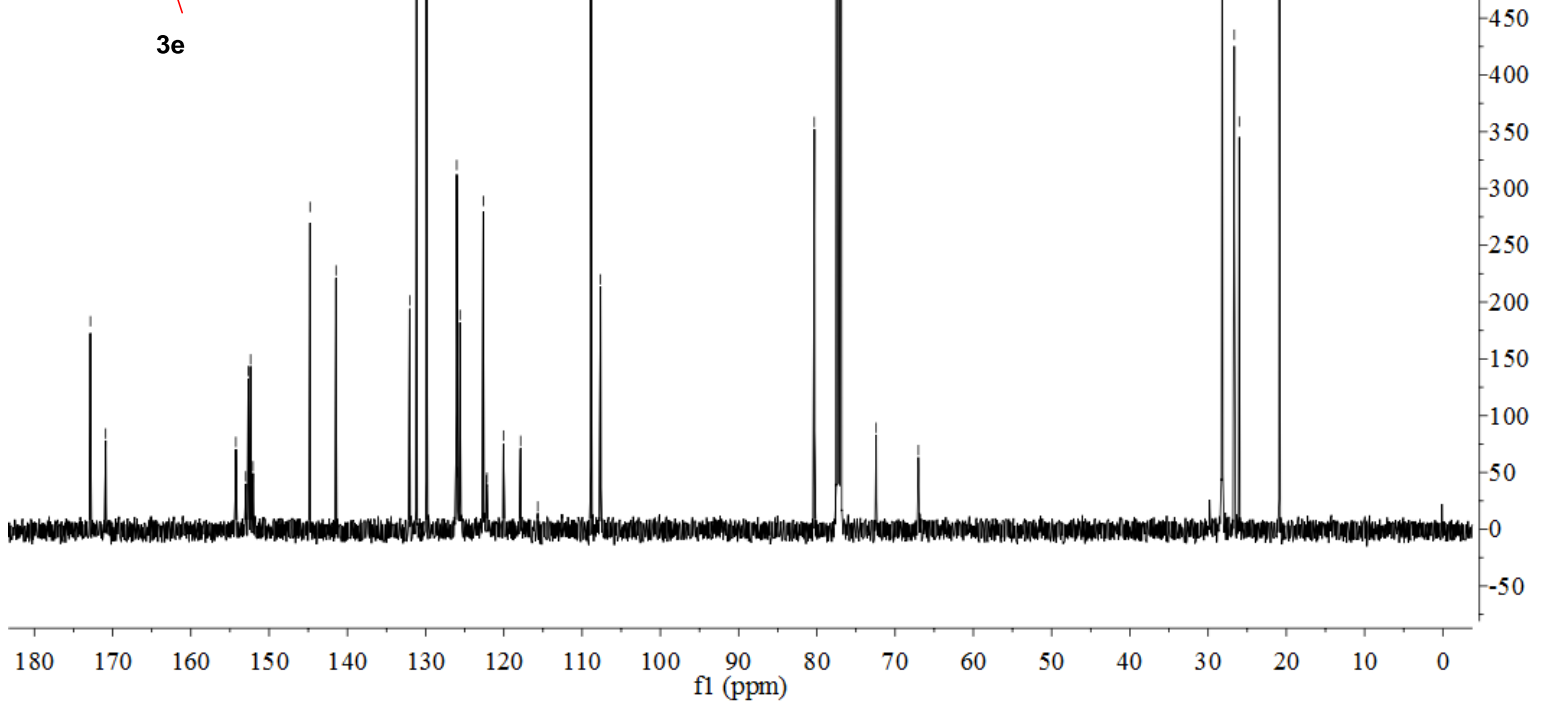


${ }^{19} \mathrm{~F} \mathrm{NMR}$ of compound $3 \mathrm{e}\left(376 \mathrm{MHz}\right.$ in $\left.\mathrm{CDCl}_{3}\right)$

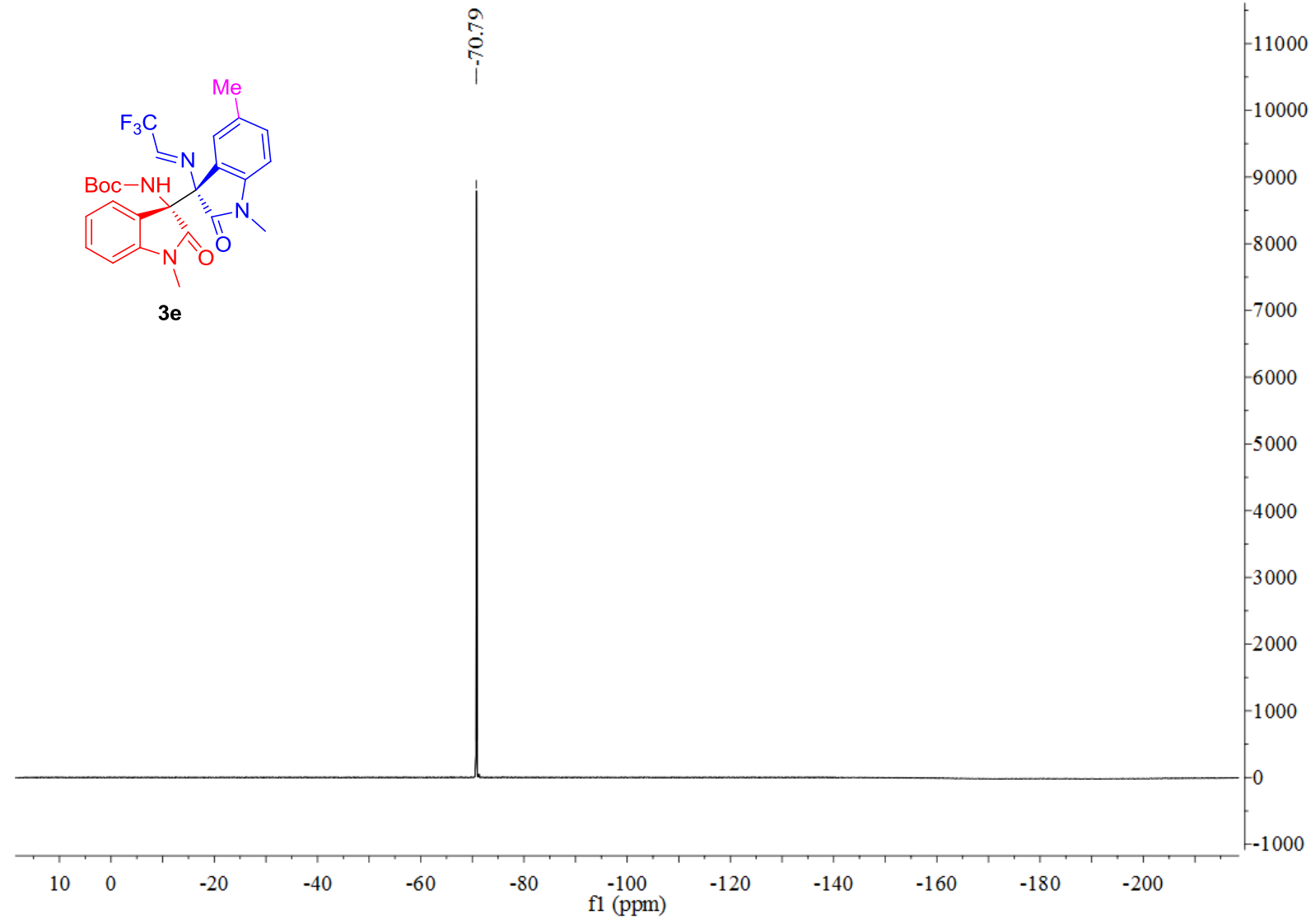

${ }^{1} \mathrm{H}$ NMR of compound $3 f\left(400 \mathrm{MHz}\right.$ in $\mathrm{CDCl}_{3}$ )

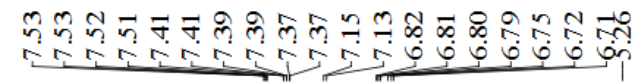

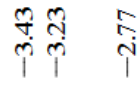

$\stackrel{\text { ก }}{i}$

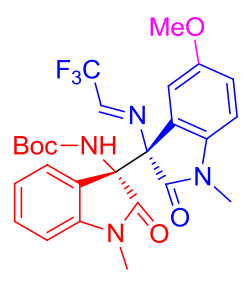

$3 f$

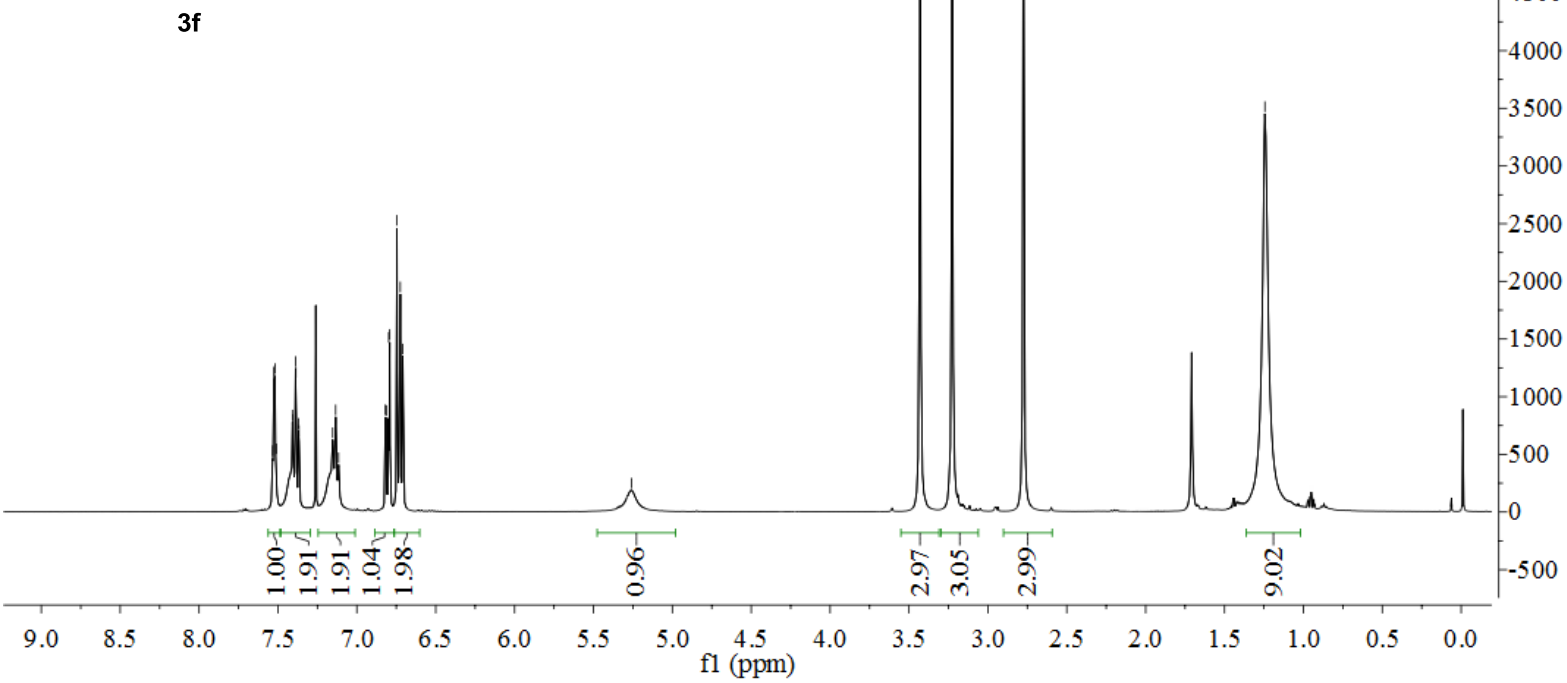


${ }^{13} \mathrm{C}$ NMR of compound $3 \mathrm{f}\left(101 \mathrm{MHz}\right.$ in $\left.\mathrm{CDCl}_{3}\right)$

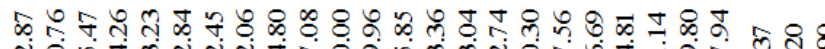

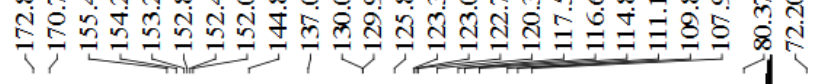

त)

$\frac{10}{\infty}=\frac{1}{0}$

6000

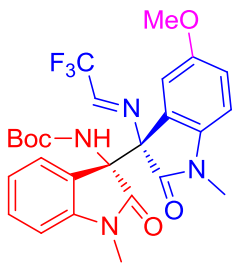

$3 f$

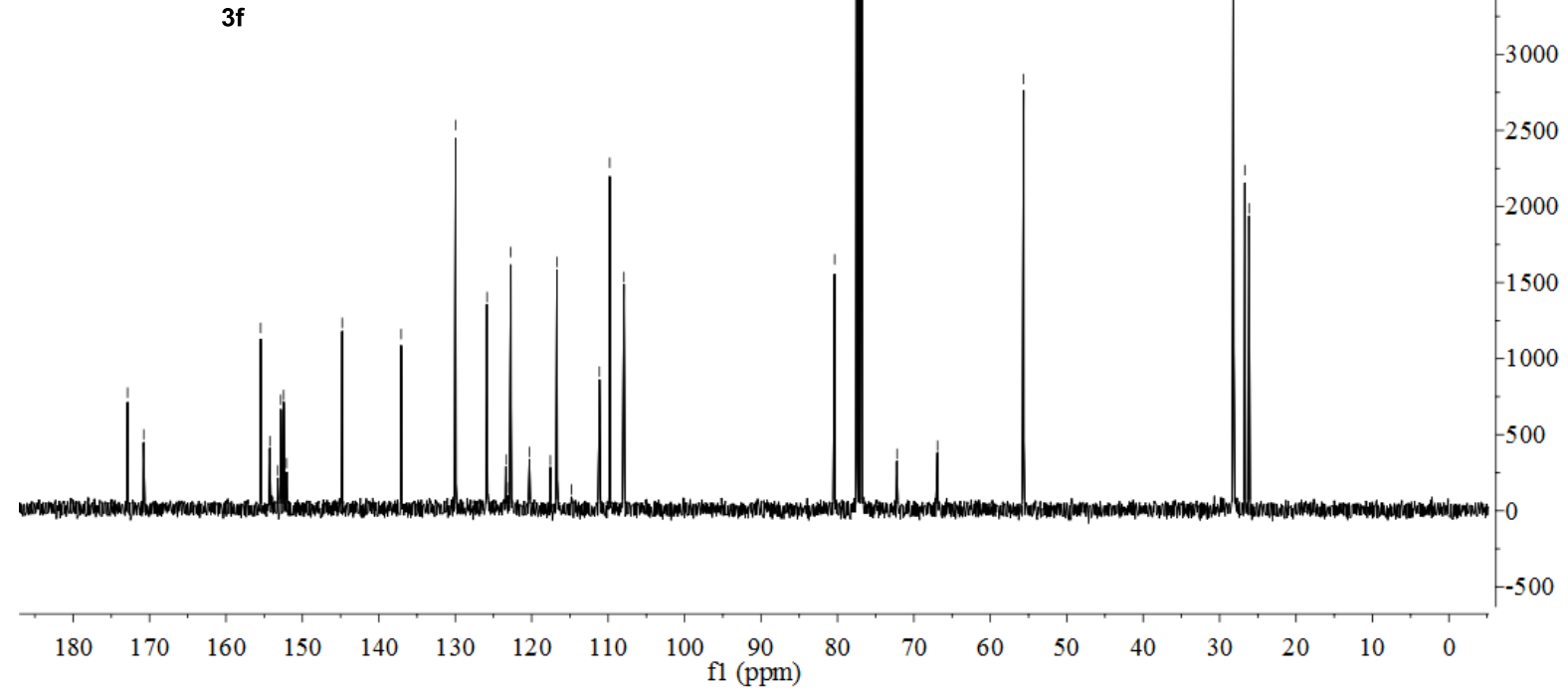

${ }^{19} \mathrm{~F} \mathrm{NMR}$ of compound $3 f\left(376 \mathrm{MHz}\right.$ in $\left.\mathrm{CDCl}_{3}\right)$

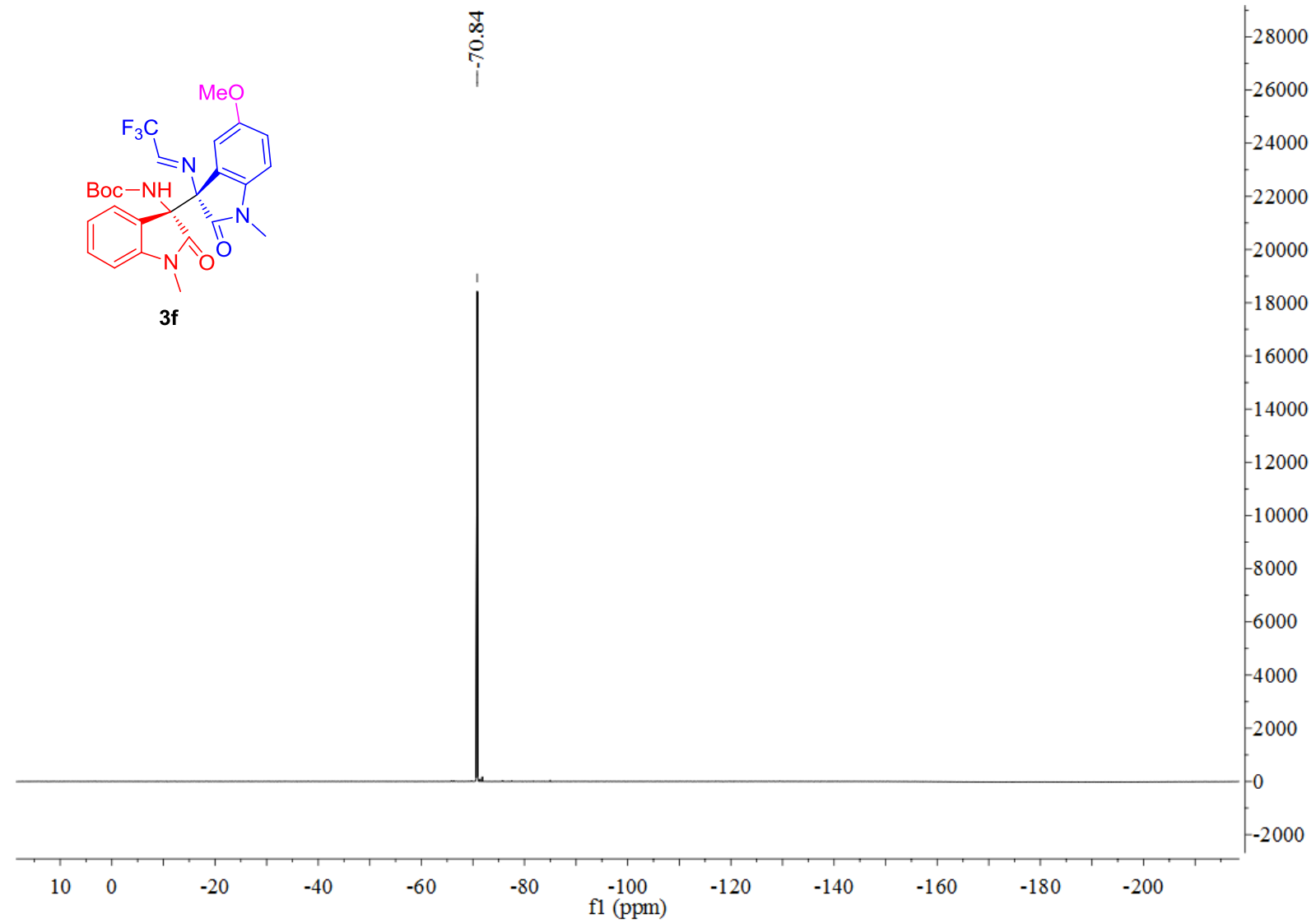


${ }^{1} \mathrm{H}$ NMR of compound $3 \mathrm{~g}\left(400 \mathrm{MHz}\right.$ in $\left.\mathrm{CDCl}_{3}\right)$

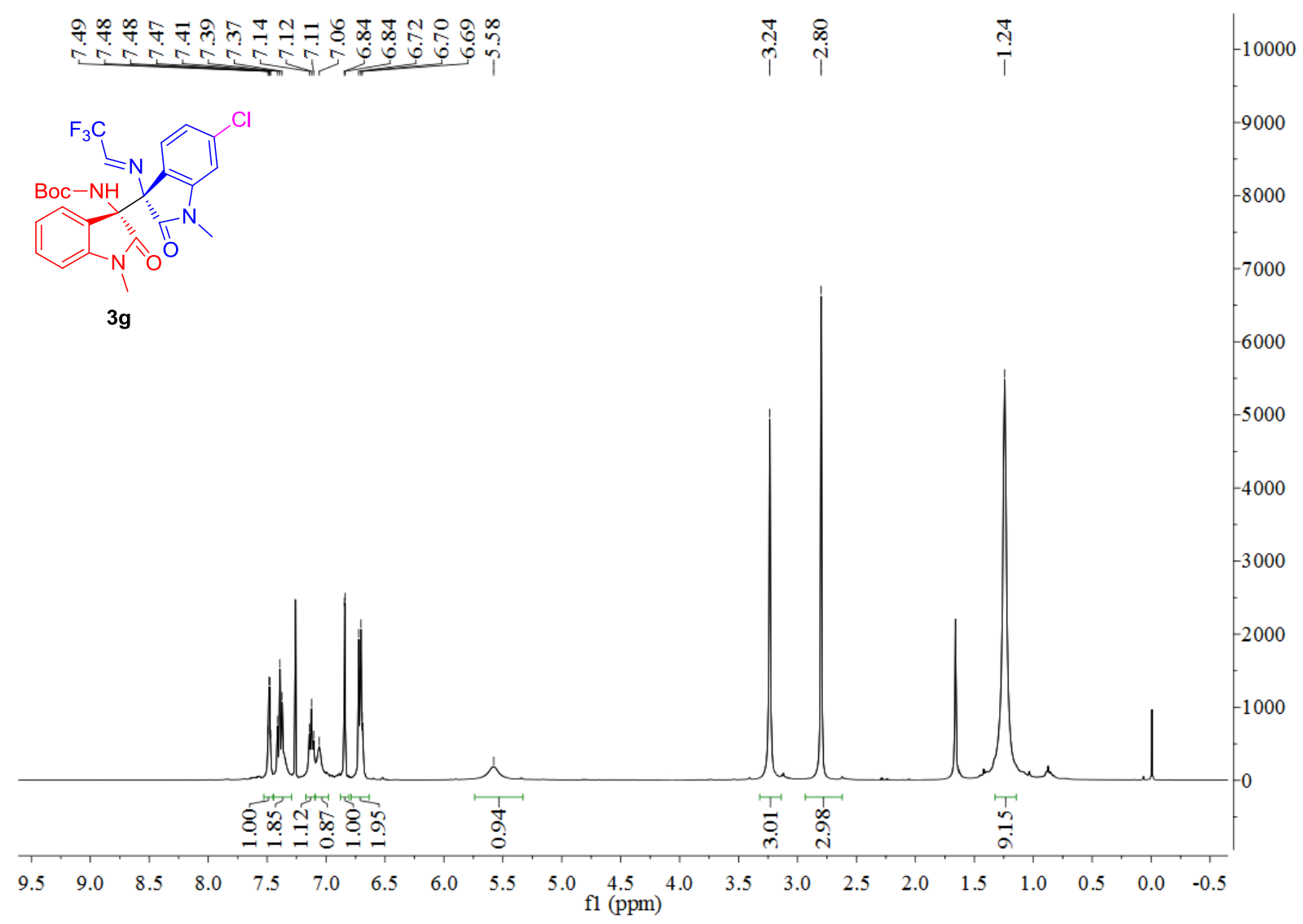

${ }^{13} \mathrm{C}$ NMR of compound $3 \mathrm{~g}\left(101 \mathrm{MHz}\right.$ in $\left.\mathrm{CDCl}_{3}\right)$

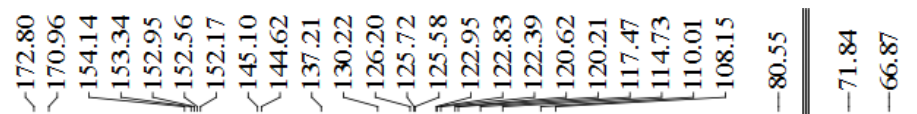

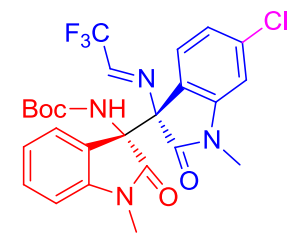

3g

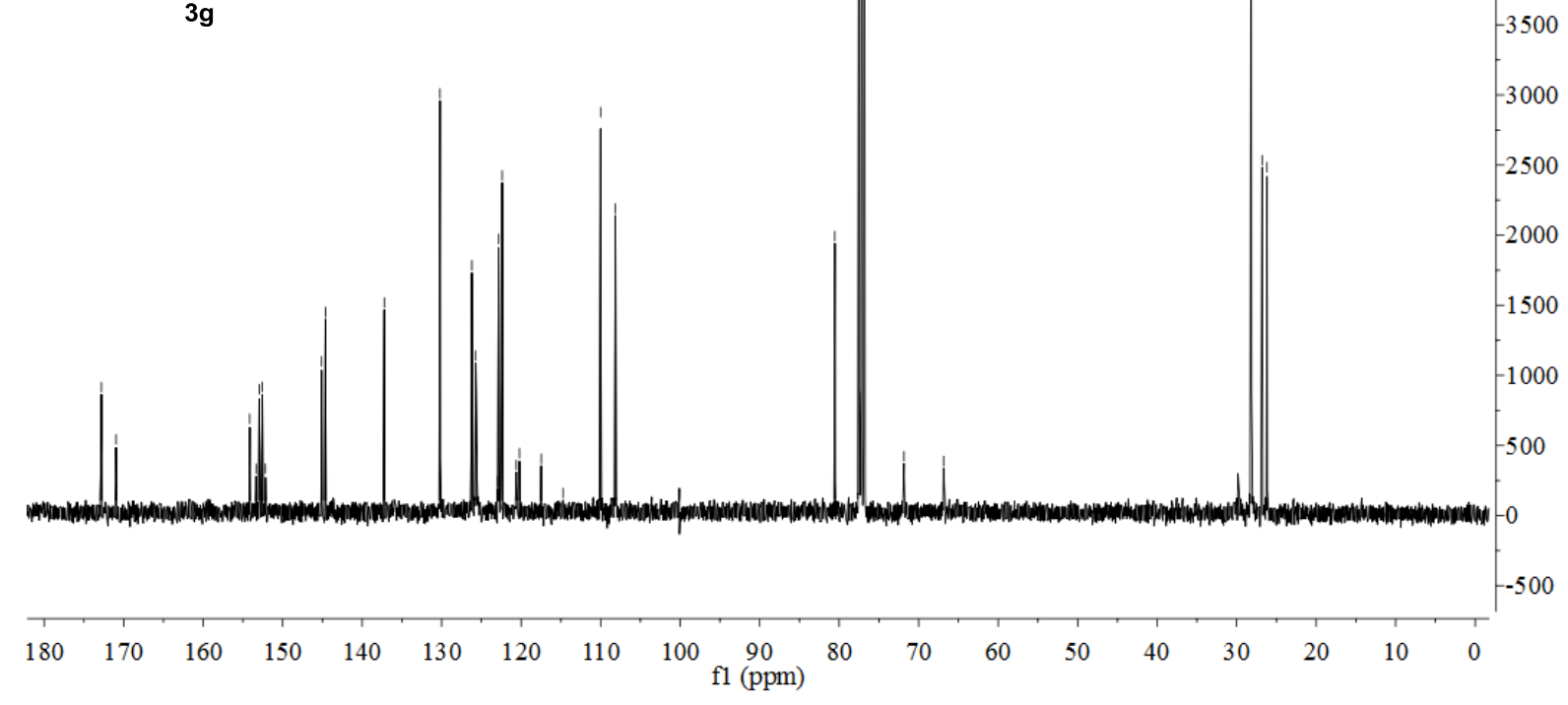


${ }^{19} \mathrm{~F} \mathrm{NMR}$ of compound $3 \mathrm{~g}\left(376 \mathrm{MHz}\right.$ in $\mathrm{CDCl}_{3}$ )

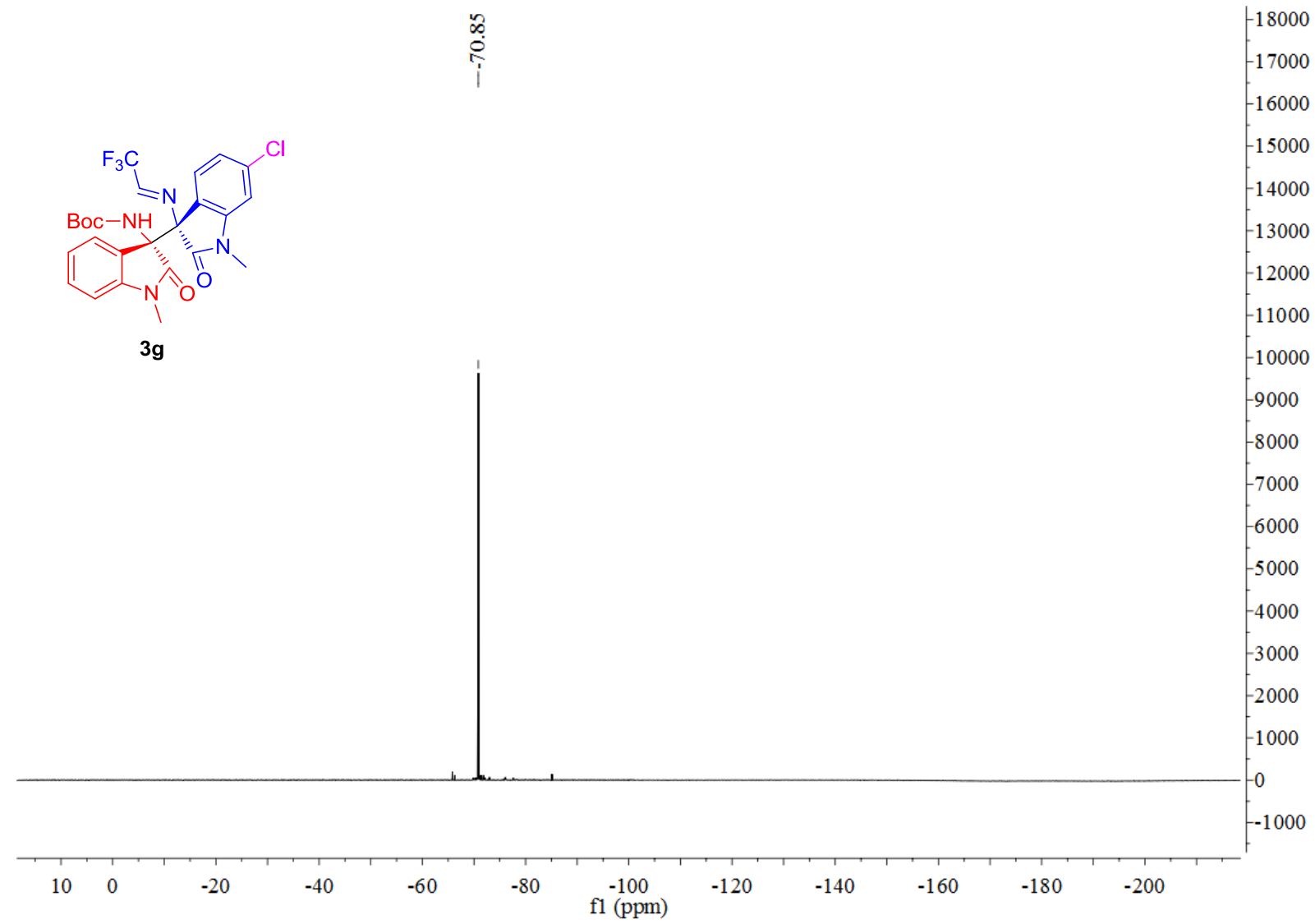

${ }^{1} \mathrm{H}$ NMR of compound $3 \mathrm{~h}\left(400 \mathrm{MHz}\right.$ in $\left.\mathrm{CDCl}_{3}\right)$

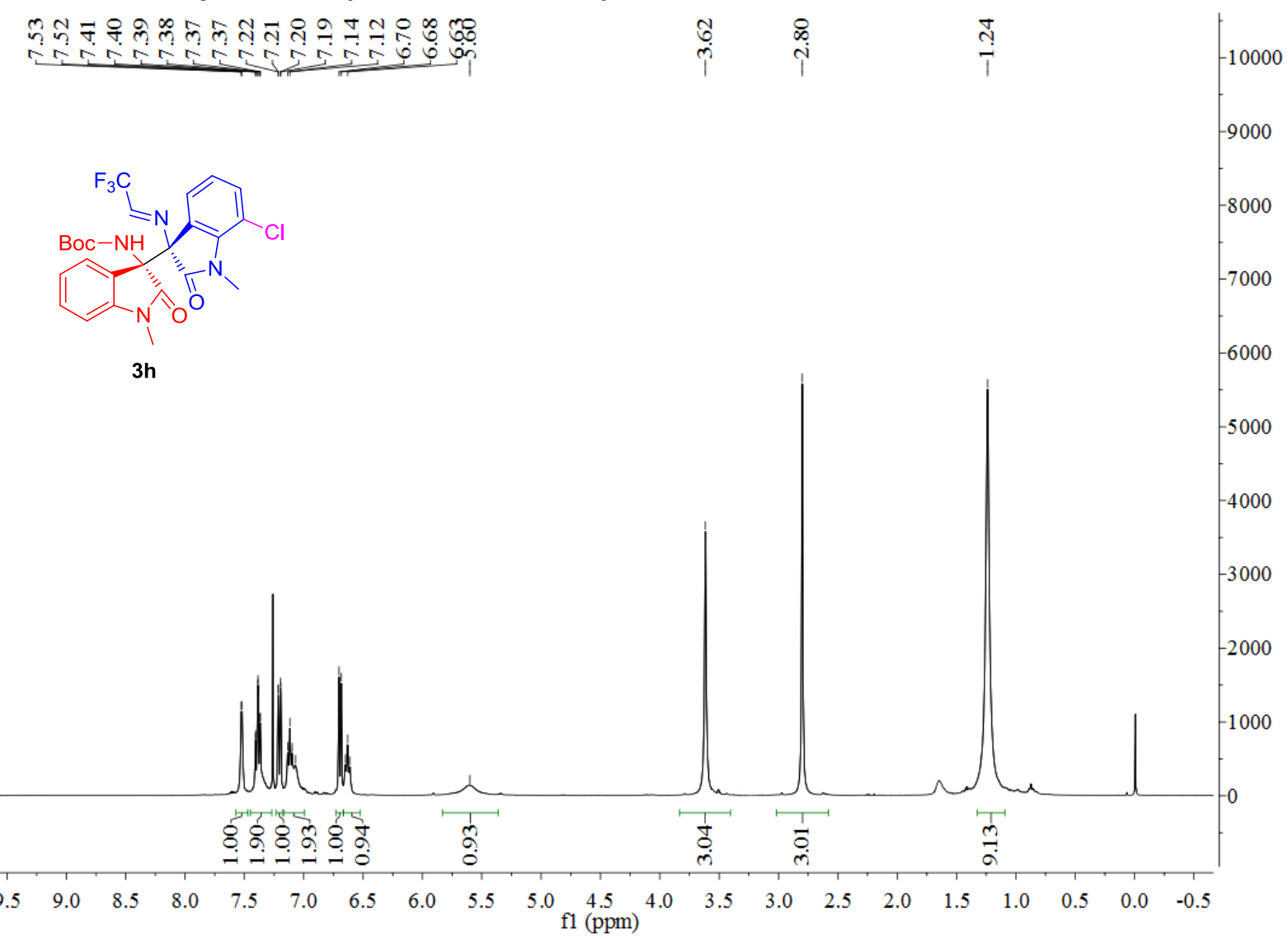


${ }^{13} \mathrm{C}$ NMR of compound $3 \mathrm{~h}\left(101 \mathrm{MHz}\right.$ in $\mathrm{CDCl}_{3}$ )

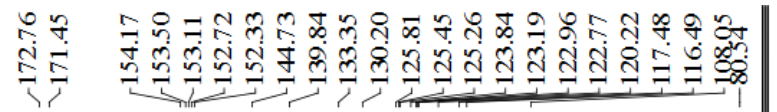

1
78
78

$\frac{d}{\infty} \frac{d}{0}$

$-5500$

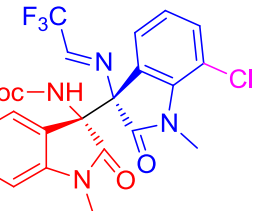

$3 \mathrm{~h}$

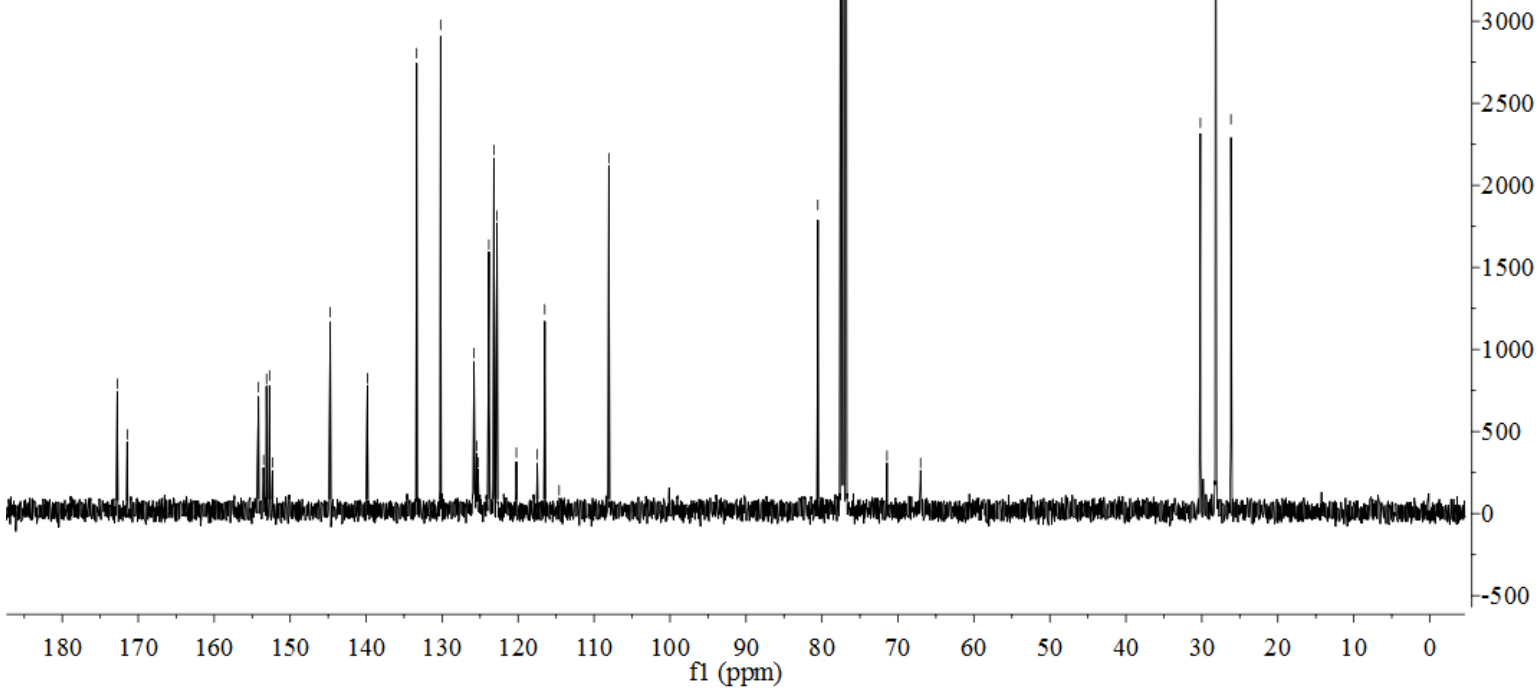

${ }^{19} \mathrm{~F} \mathrm{NMR}$ of compound $3 \mathrm{~h}\left(376 \mathrm{MHz}\right.$ in $\left.\mathrm{CDCl}_{3}\right)$

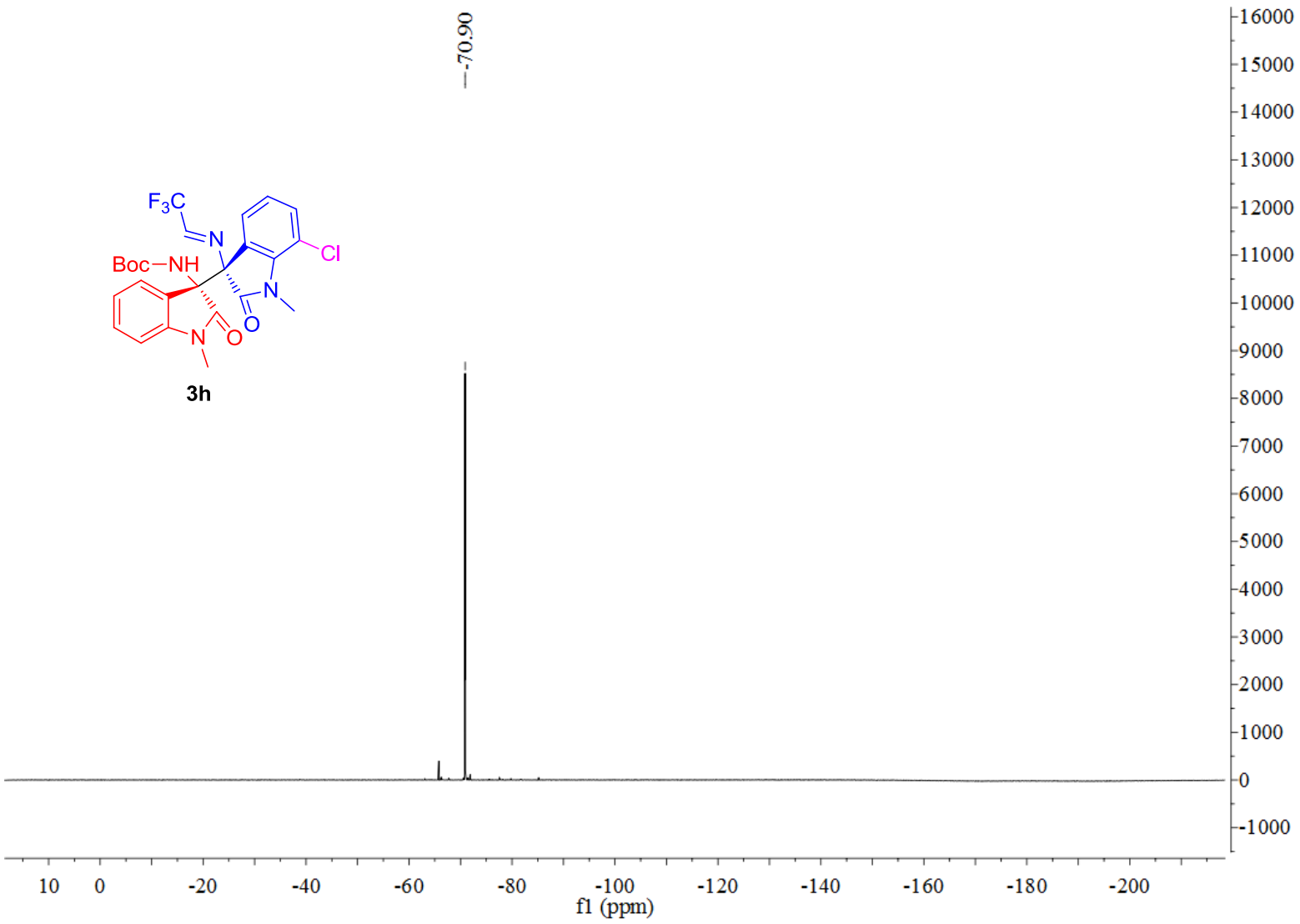


${ }^{1} \mathrm{H}$ NMR of compound $3 \mathrm{i}\left(400 \mathrm{MHz}\right.$ in $\mathrm{CDCl}_{3}$ )

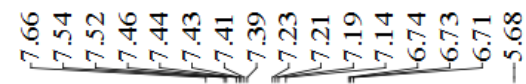

$\stackrel{\infty}{i}$

$\stackrel{1}{\stackrel{1}{4}}$

8000

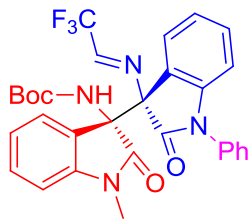

3i

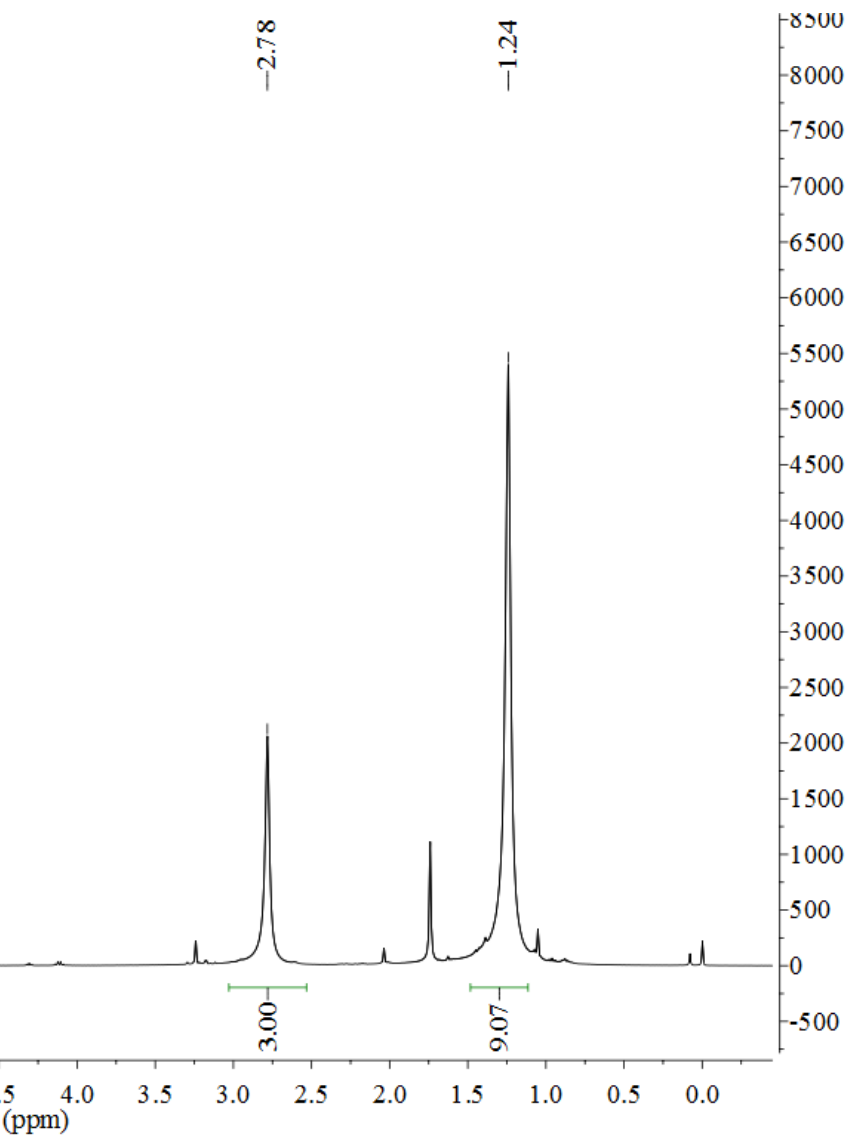

${ }^{13} \mathrm{C}$ NMR of compound $3 \mathrm{i}\left(101 \mathrm{MHz}\right.$ in $\mathrm{CDCl}_{3}$ )

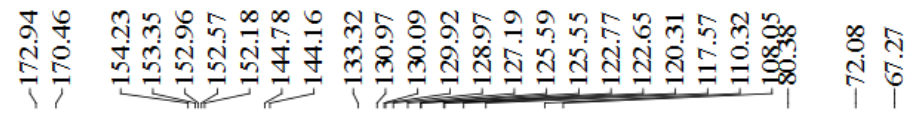

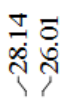

$-5500$

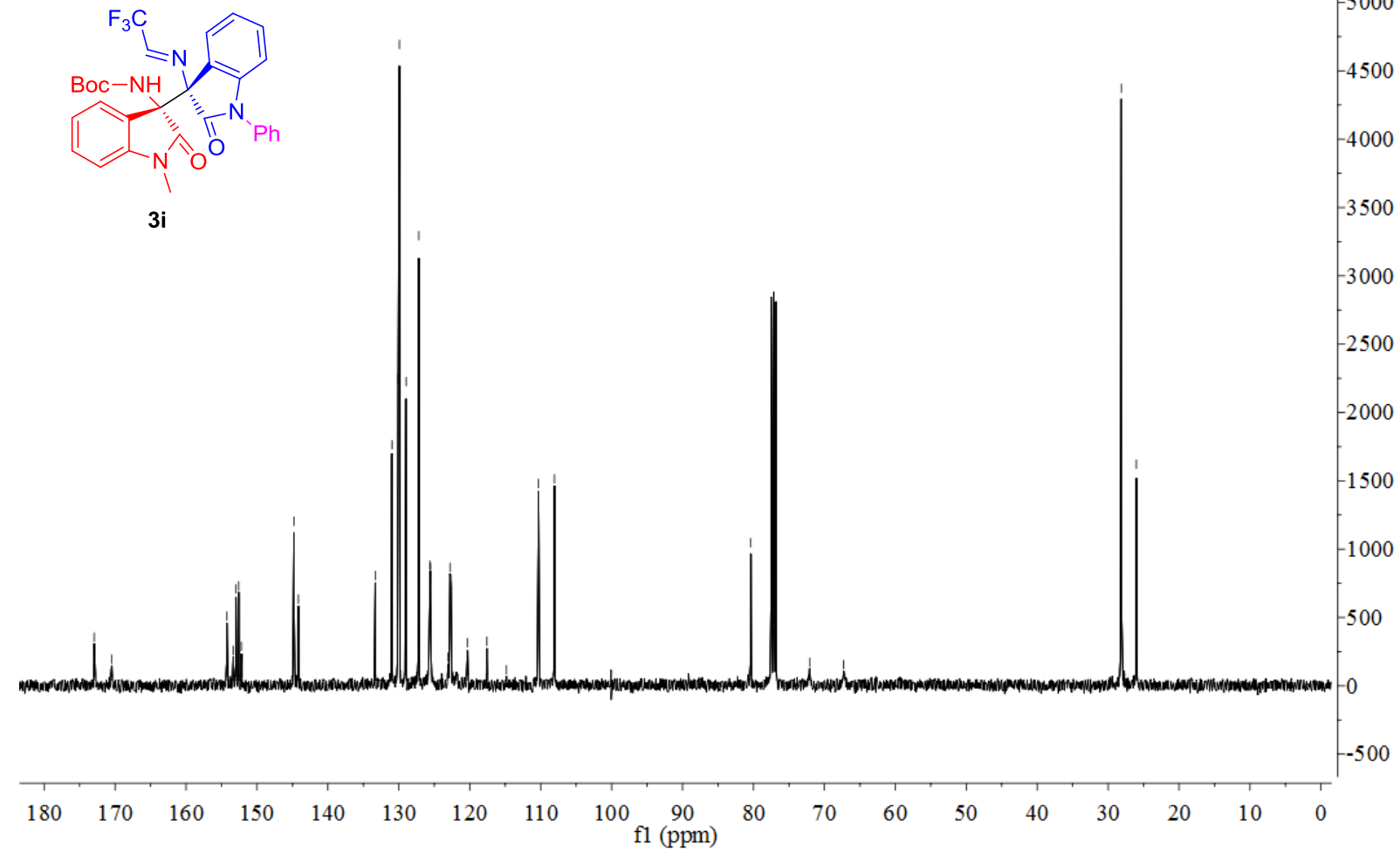

$3 i$

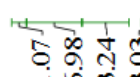
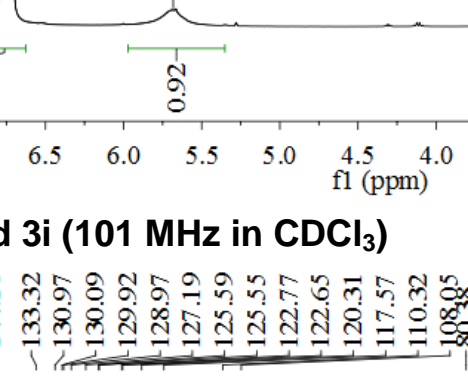

ìं

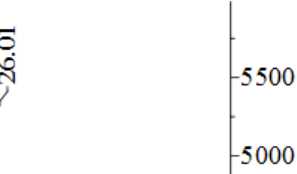


${ }^{19} \mathrm{~F} \mathrm{NMR}$ of compound $3 \mathrm{i}\left(376 \mathrm{MHz}\right.$ in $\left.\mathrm{CDCl}_{3}\right)$

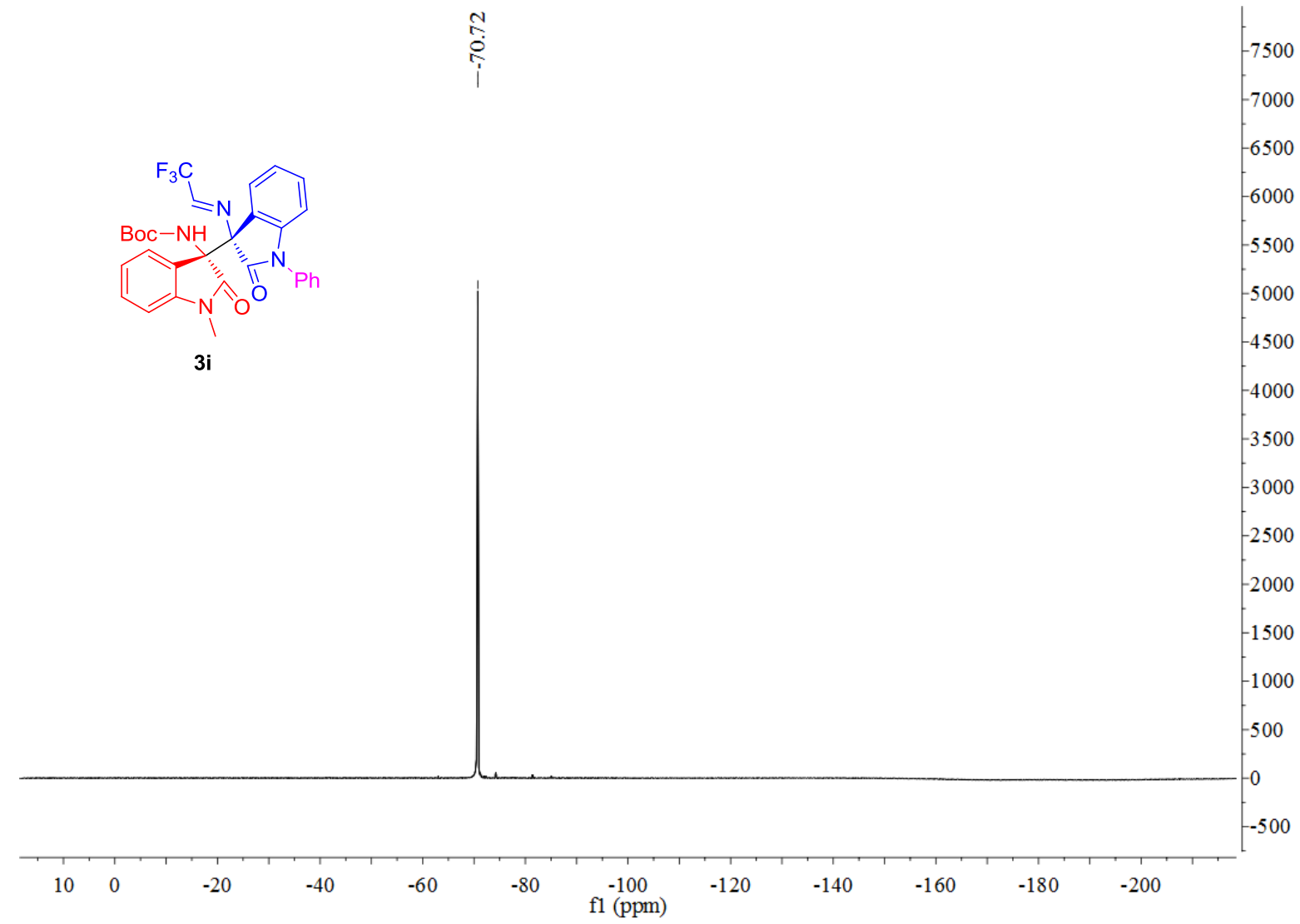

${ }^{1} \mathrm{H}$ NMR of compound $3 \mathrm{j}$ (400 $\mathrm{MHz}$ in $\mathrm{CDCl}_{3}$ )

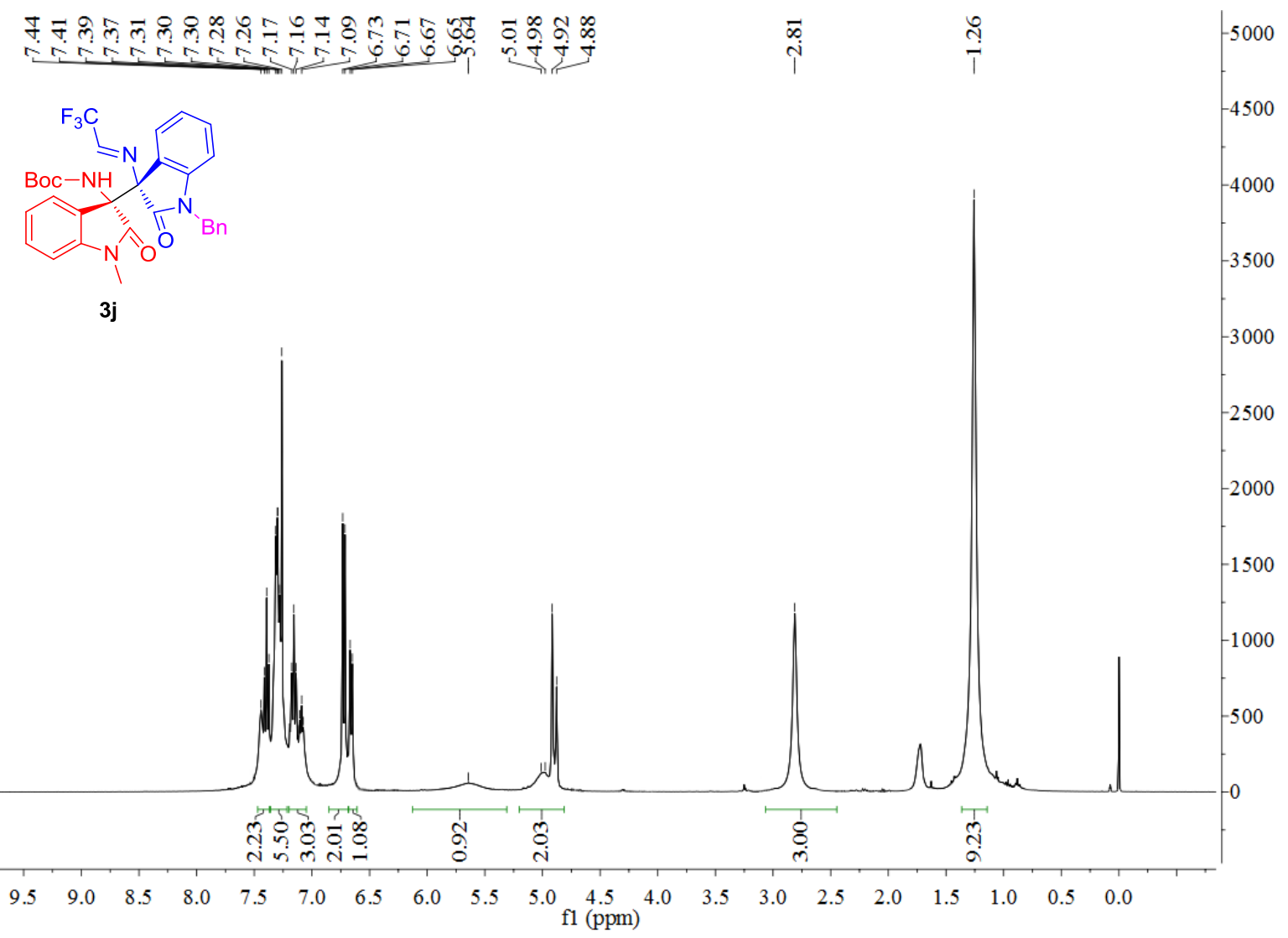


${ }^{13} \mathrm{C}$ NMR of compound $3 \mathrm{j}\left(126 \mathrm{MHz}\right.$ in $\mathrm{CDCl}_{3}$ )

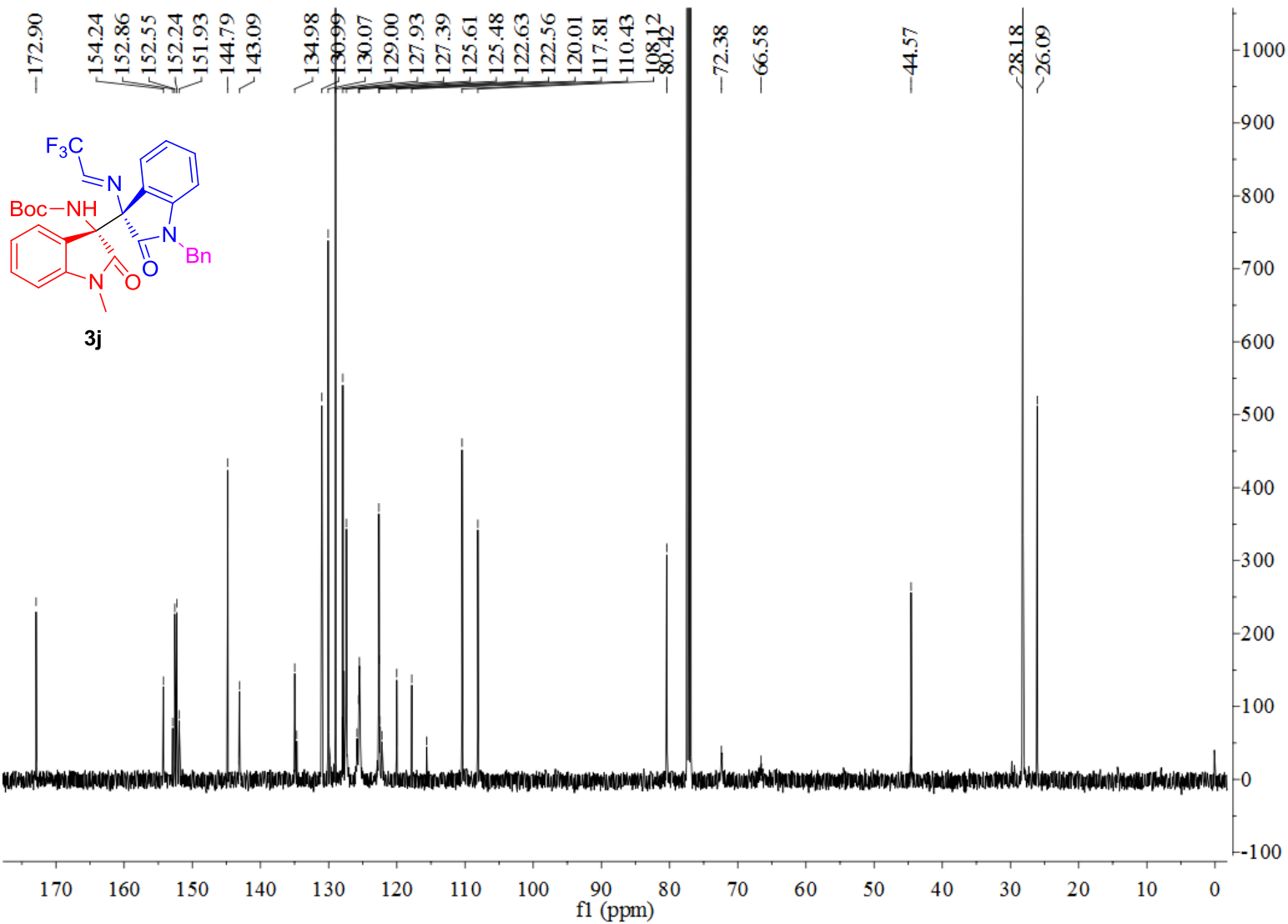

${ }^{19} \mathrm{~F}$ NMR of compound $3 \mathrm{j}\left(376 \mathrm{MHz}\right.$ in $\mathrm{CDCl}_{3}$ )

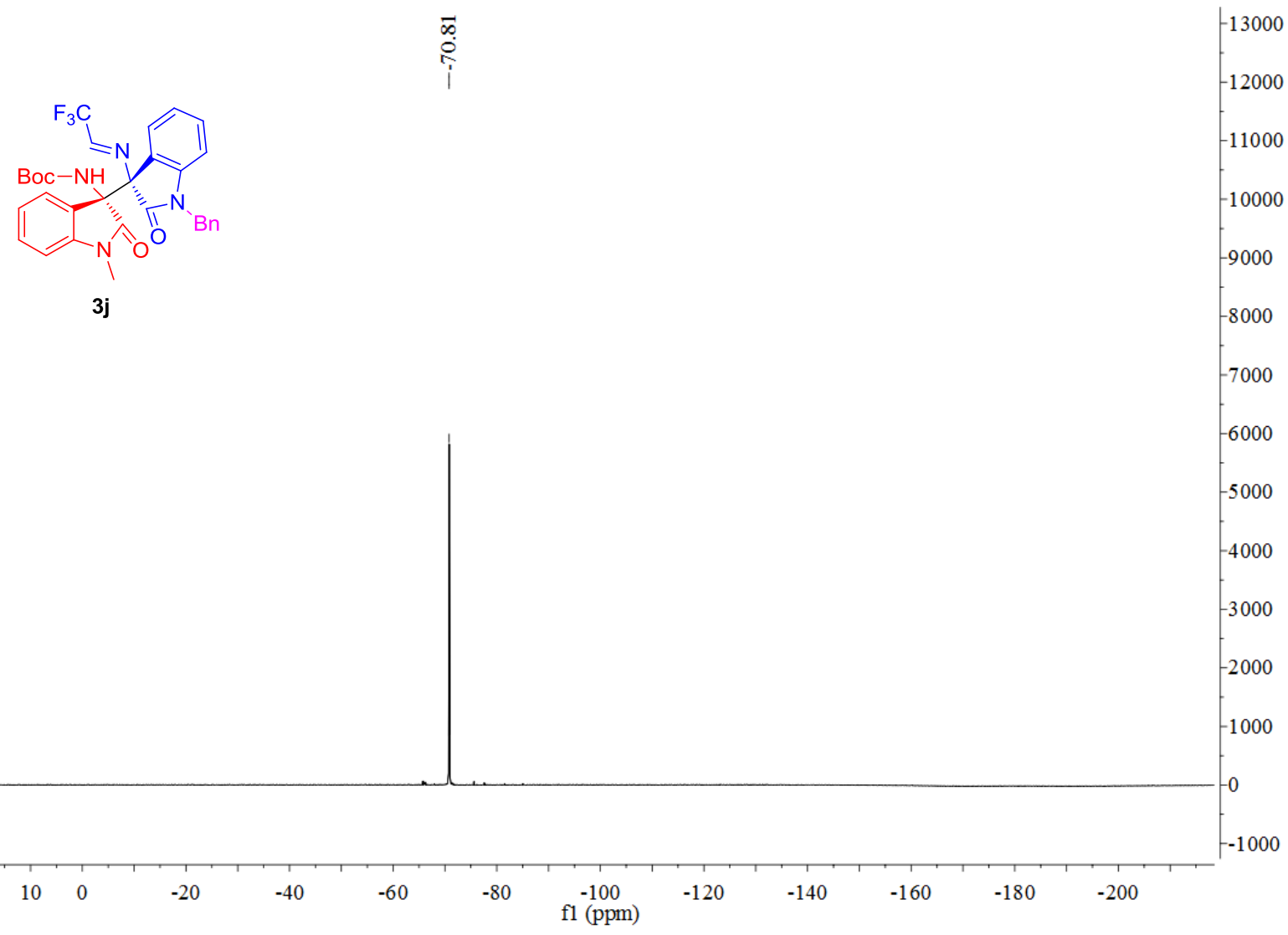


${ }^{1} \mathrm{H}$ NMR of compound $3 \mathrm{k}\left(400 \mathrm{MHz}\right.$ in $\mathrm{CDCl}_{3}$ )

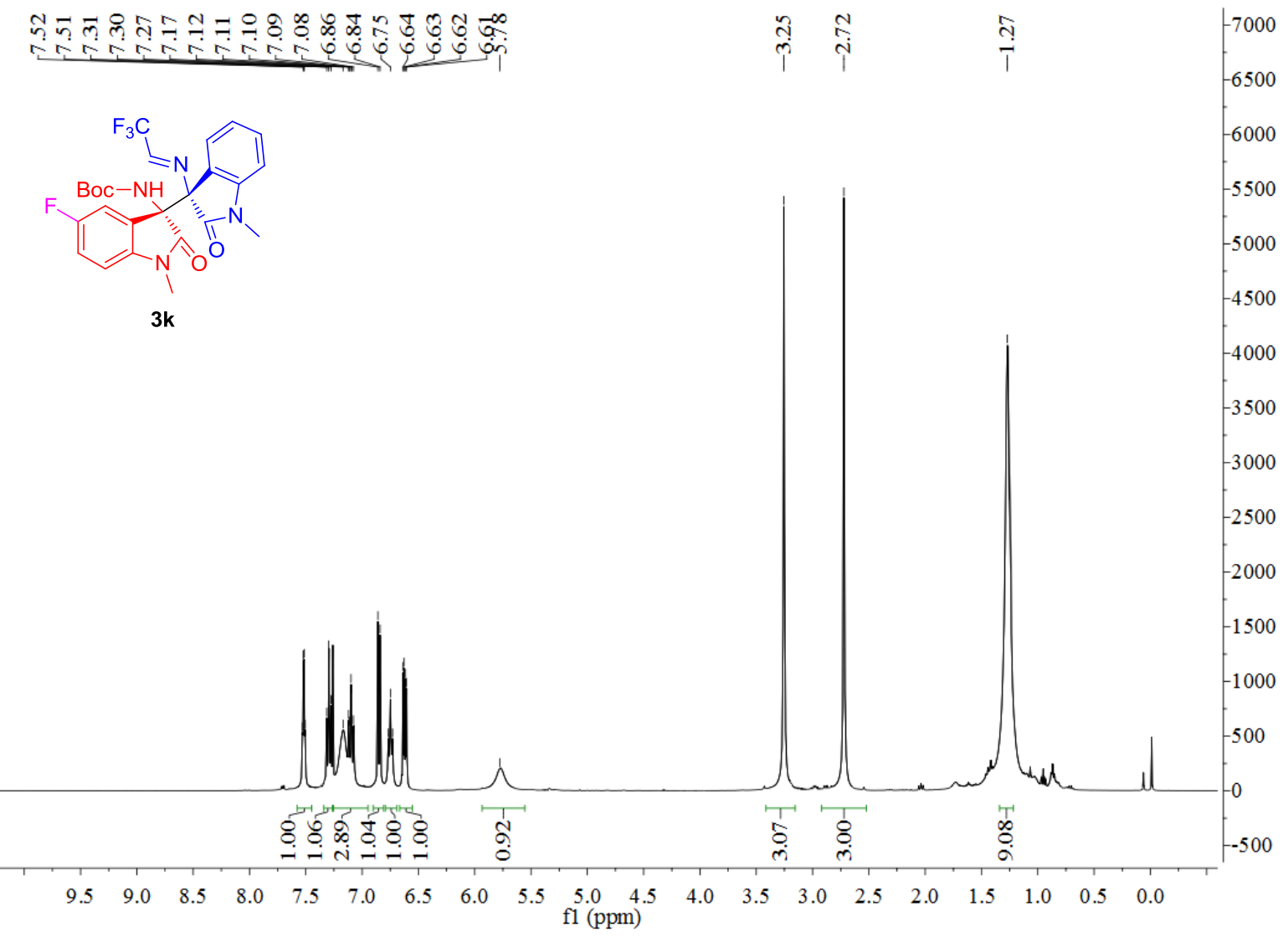

${ }^{13} \mathrm{C}$ NMR of compound 3k (126 $\mathrm{MHz}$ in $\mathrm{CDCl}_{3}$ )

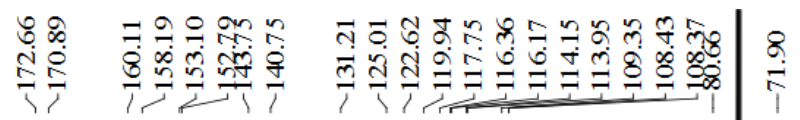

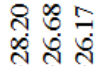

$-2200$

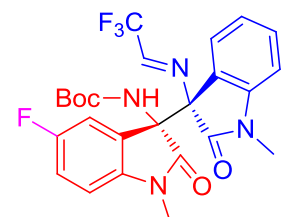

3k

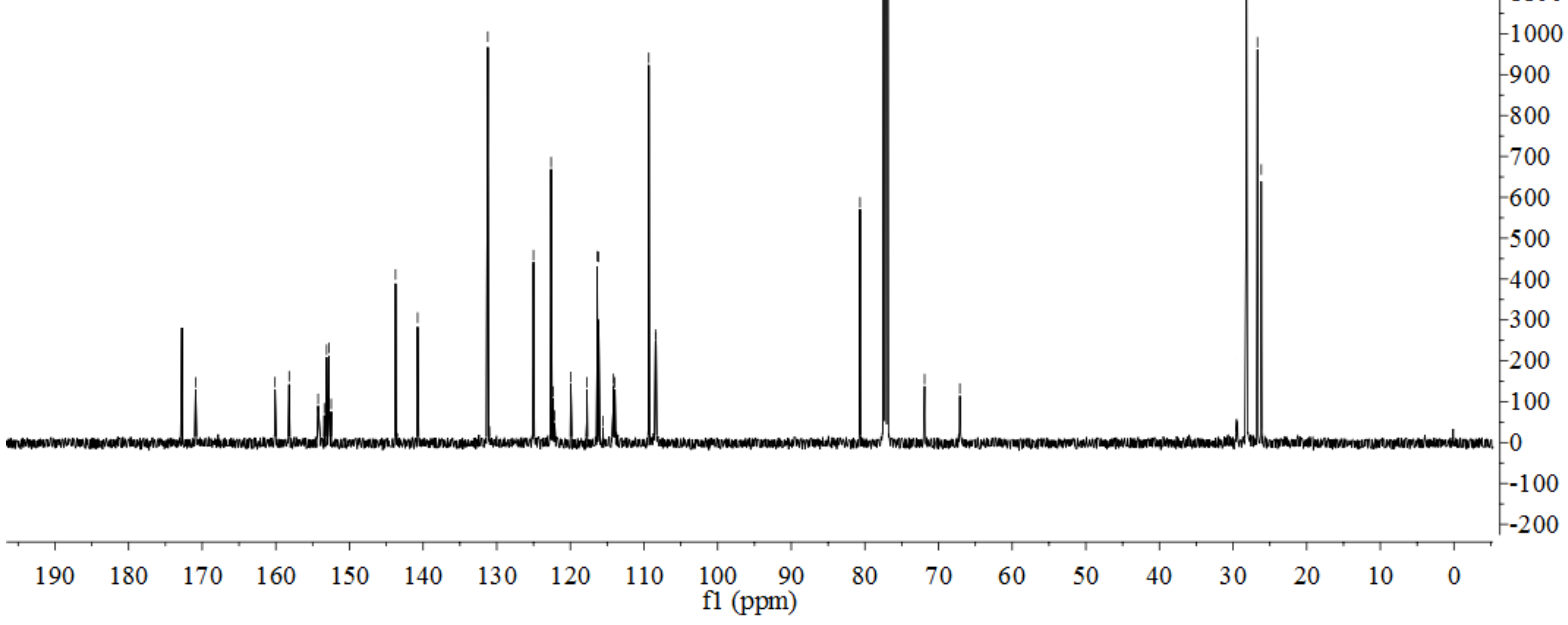


${ }^{19} \mathrm{~F} \mathrm{NMR}$ of compound $3 \mathrm{k}\left(376 \mathrm{MHz}\right.$ in $\left.\mathrm{CDCl}_{3}\right)$

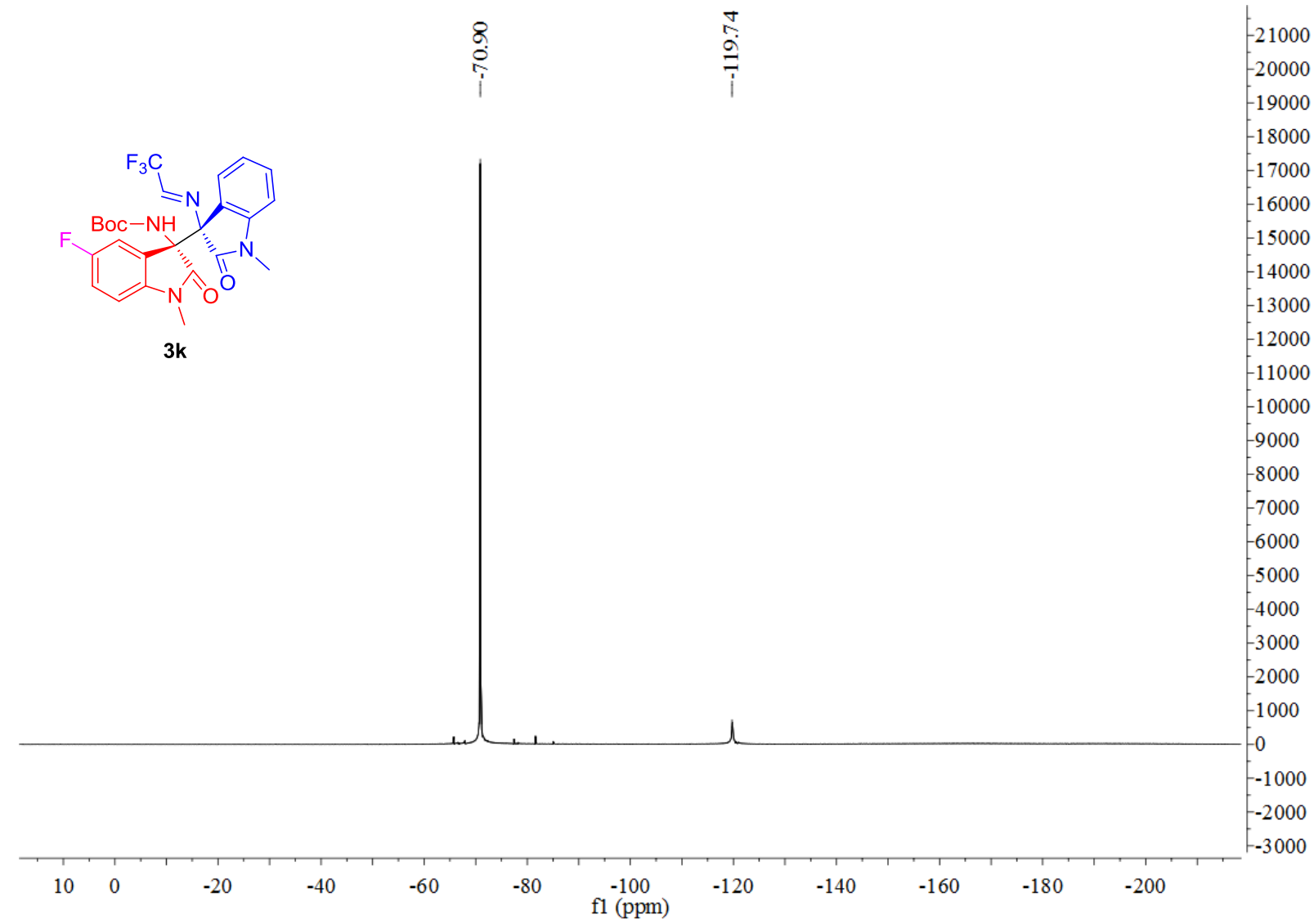

${ }^{1} \mathrm{H}$ NMR of compound $3 \mathrm{l}\left(400 \mathrm{MHz}\right.$ in $\mathrm{CDCl}_{3}$ )

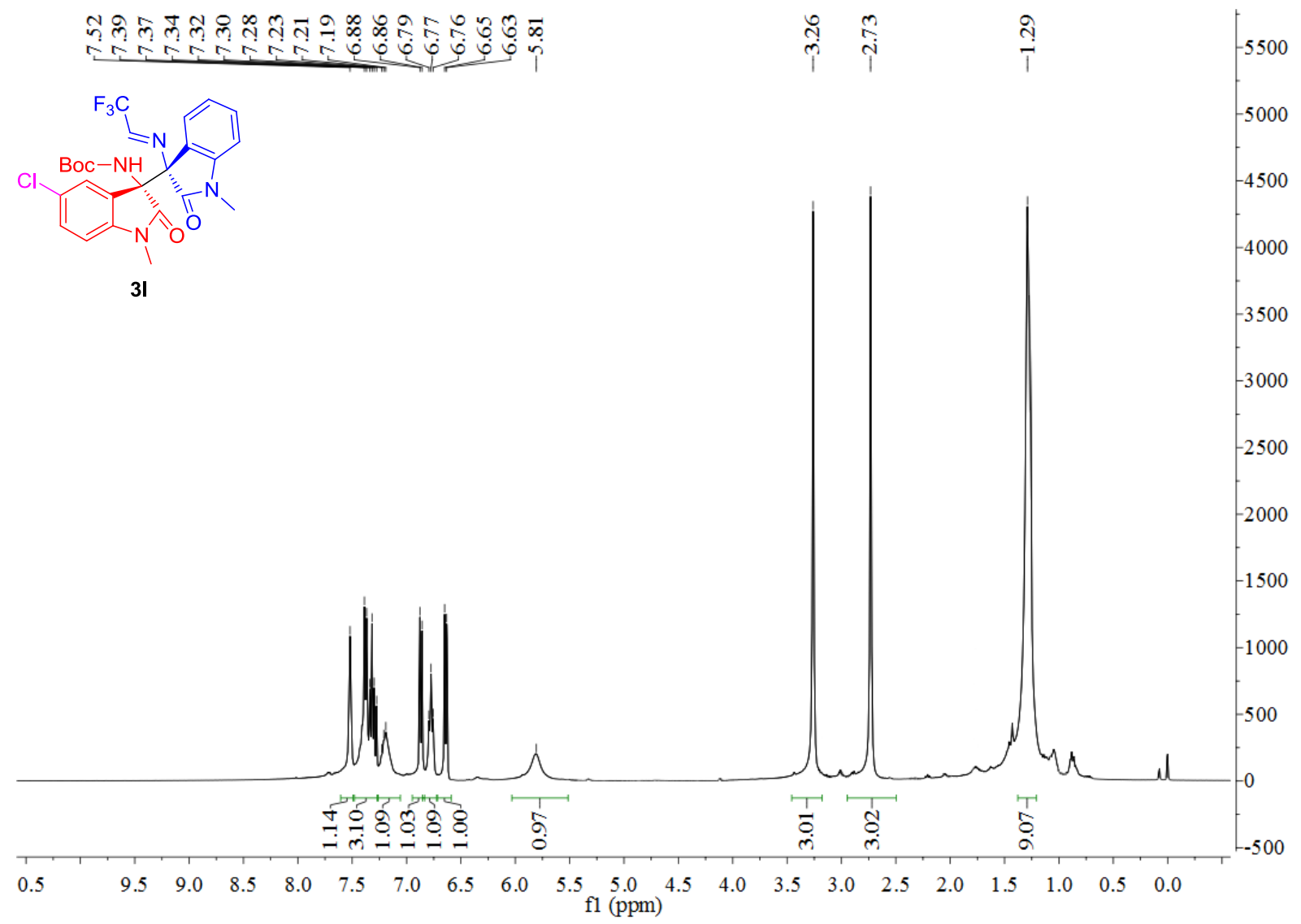


${ }^{13} \mathrm{C}$ NMR of compound $3 \mathrm{l}$ (126 $\mathrm{MHz}$ in $\mathrm{CDCl}_{3}$ )

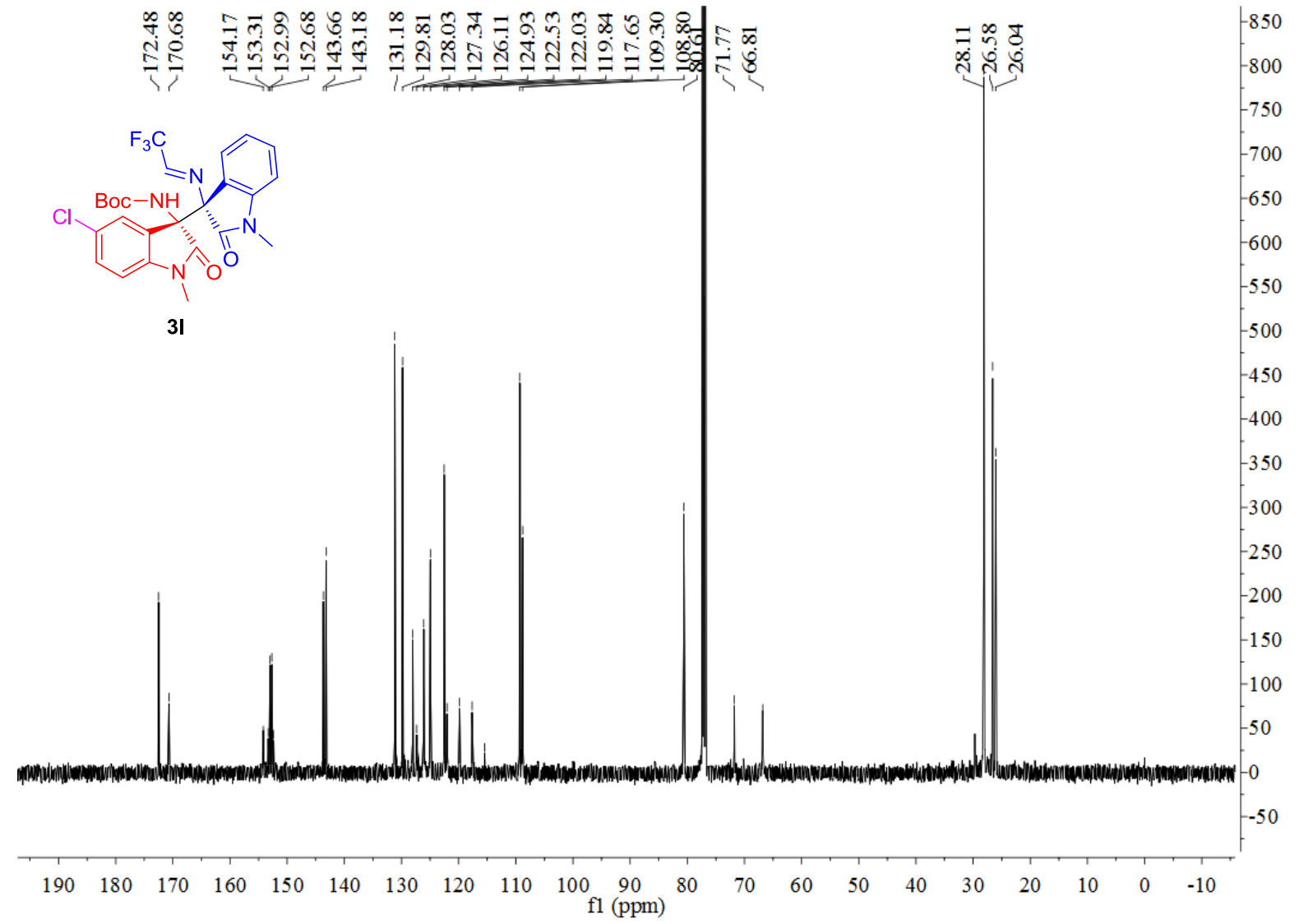

${ }^{19} \mathrm{~F} \mathrm{NMR}$ of compound $3 \mathrm{l}\left(376 \mathrm{MHz}\right.$ in $\mathrm{CDCl}_{3}$ )

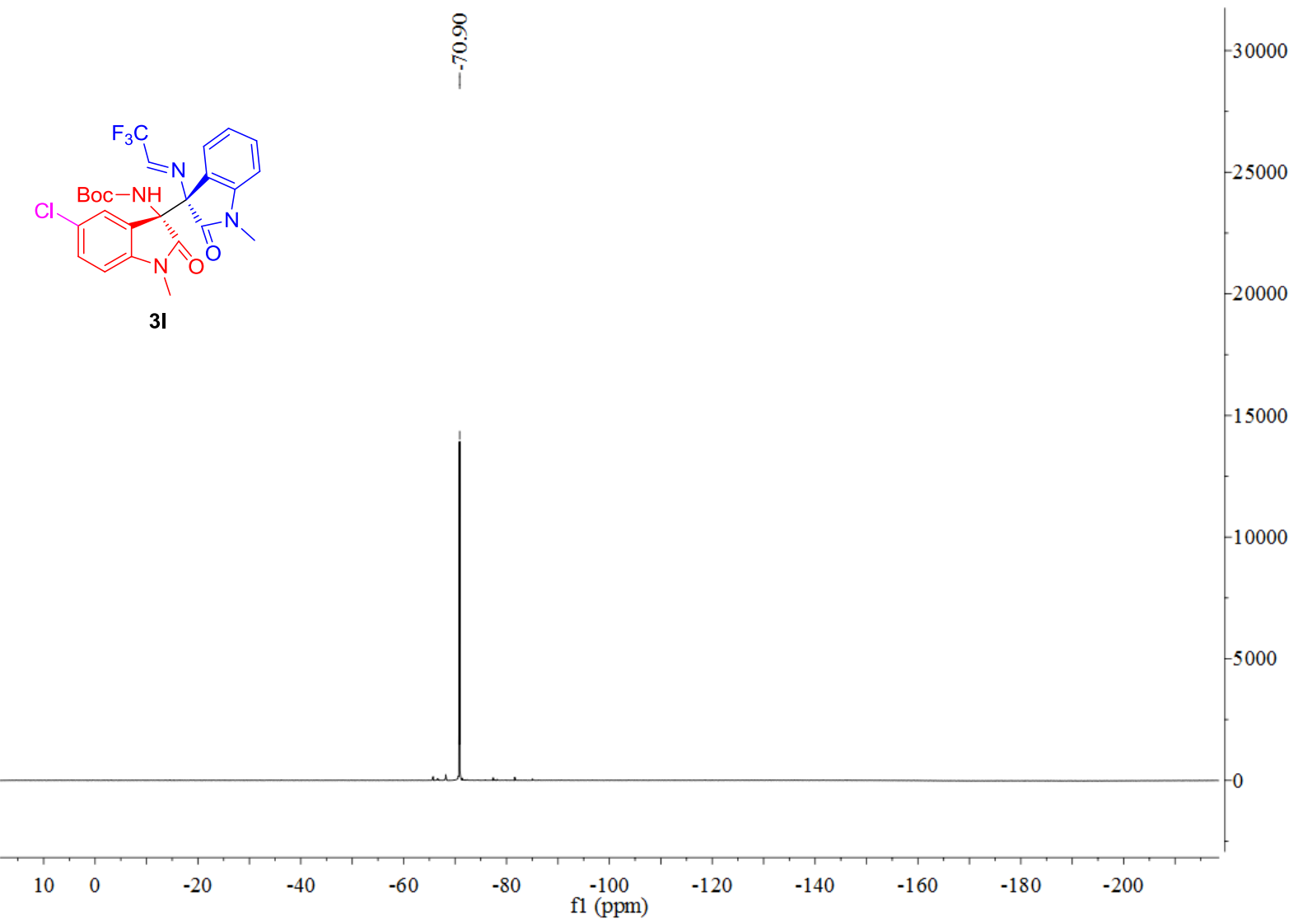


${ }^{1} \mathrm{H}$ NMR of compound $3 \mathrm{~m}\left(400 \mathrm{MHz}\right.$ in $\left.\mathrm{CDCl}_{3}\right)$

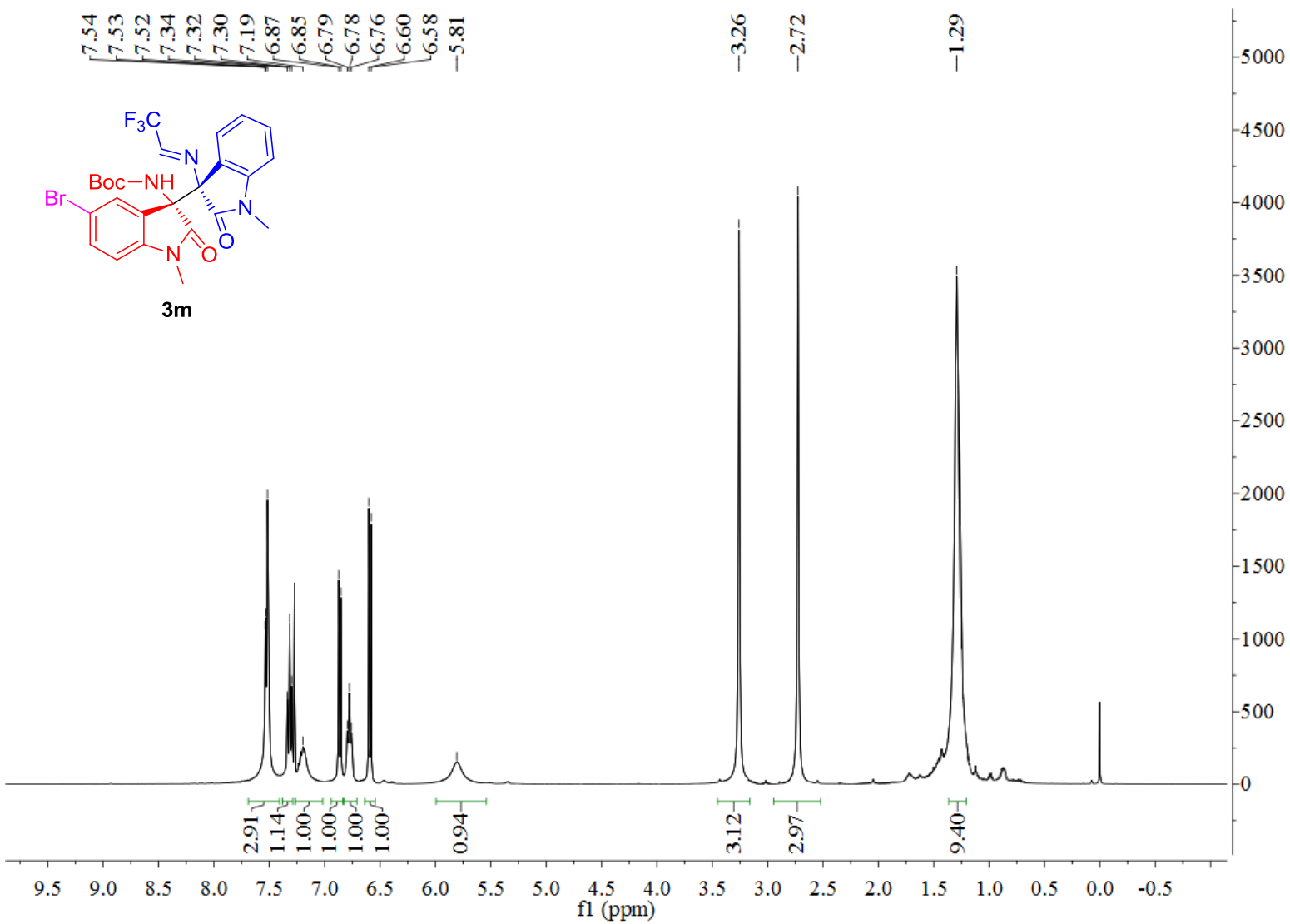

${ }^{13} \mathrm{C}$ NMR of compound $3 \mathrm{~m}$ (126 $\mathrm{MHz}$ in $\mathrm{CDCl}_{3}$ )

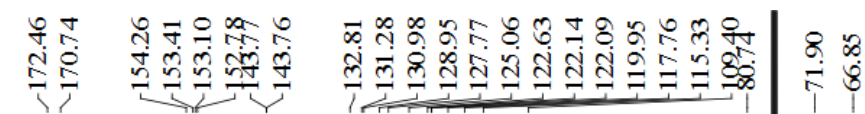

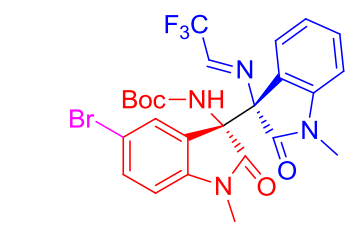

$3 m$

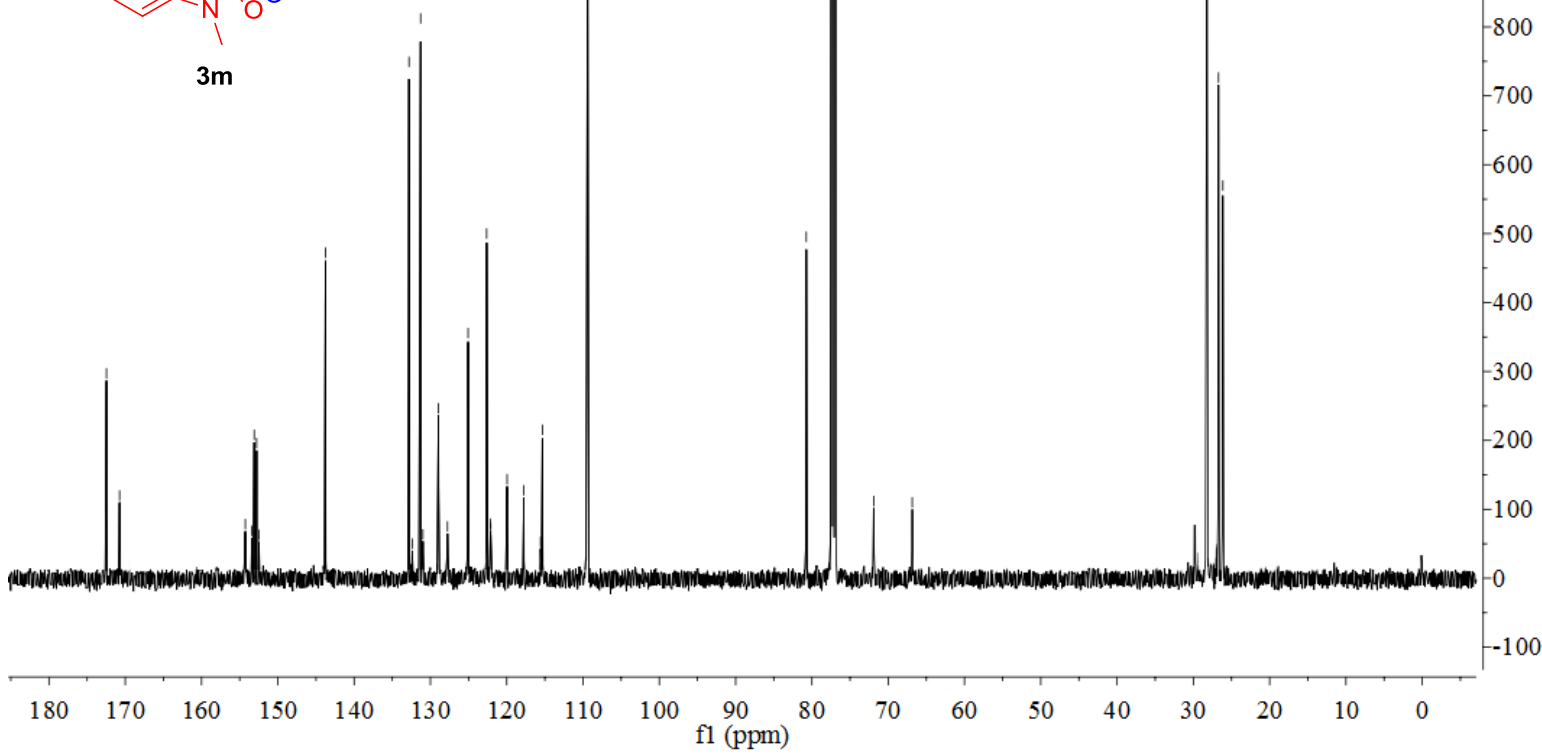


${ }^{19} \mathrm{~F}$ NMR of compound $3 \mathrm{~m}\left(376 \mathrm{MHz}\right.$ in $\left.\mathrm{CDCl}_{3}\right)$

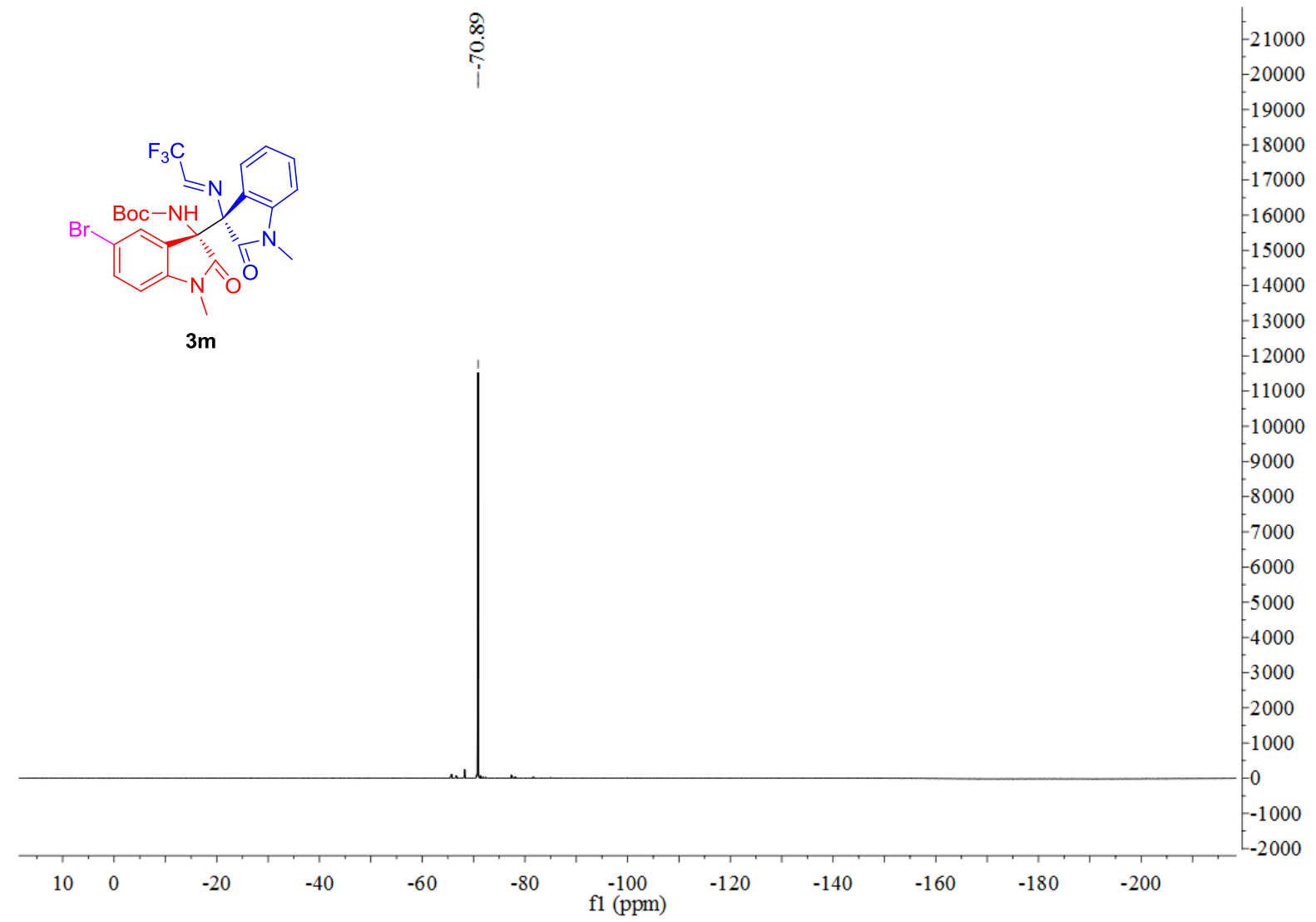

${ }^{1} \mathrm{H}$ NMR of compound $3 \mathrm{n}\left(400 \mathrm{MHz}\right.$ in $\left.\mathrm{CDCl}_{3}\right)$

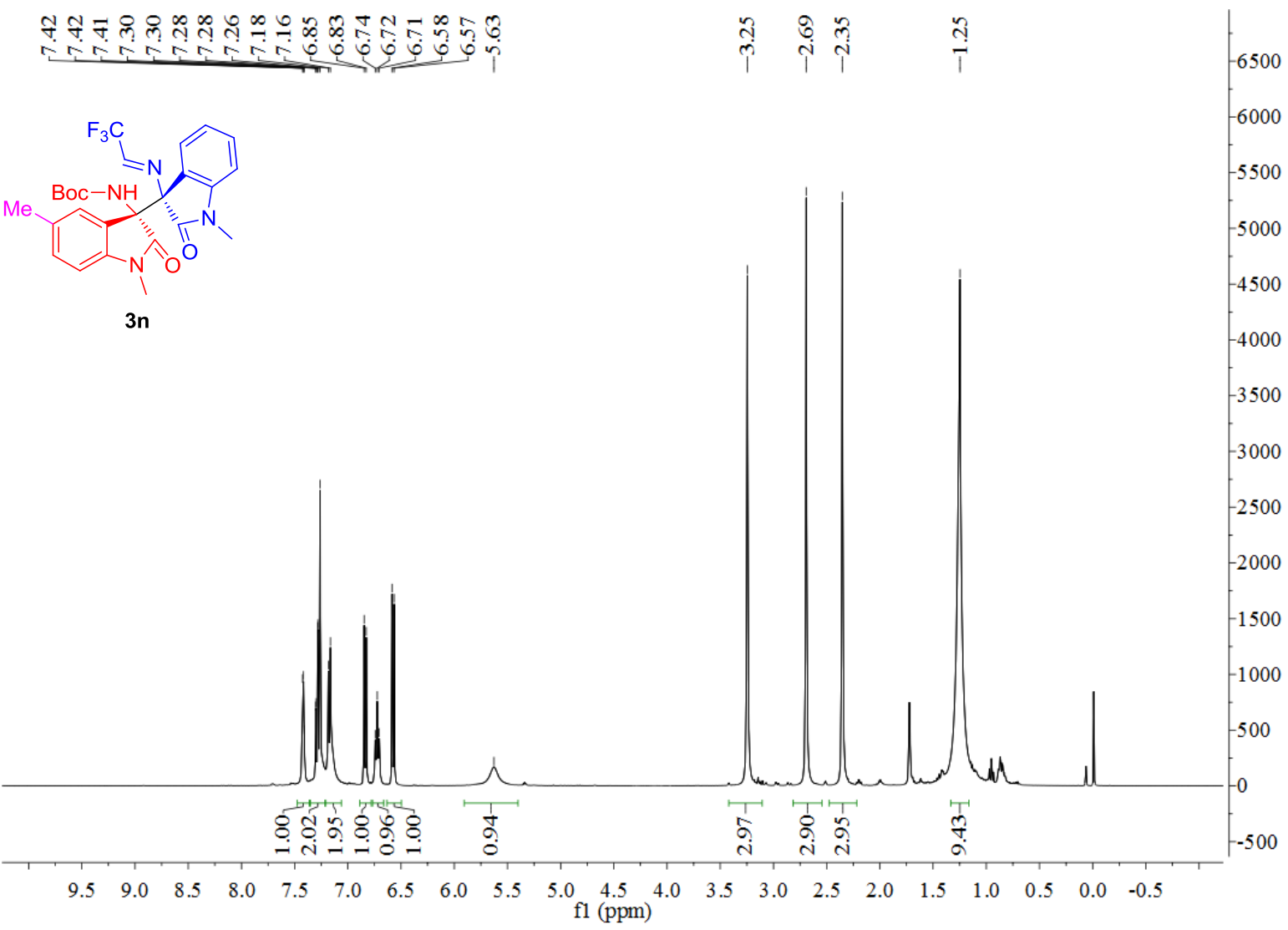


${ }^{13} \mathrm{C}$ NMR of compound 3n (101 $\mathrm{MHz}$ in $\mathrm{CDCl}_{3}$ )

भँ

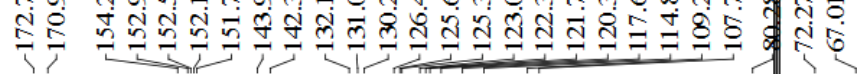

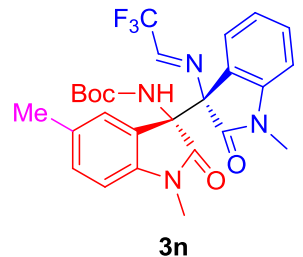

3n

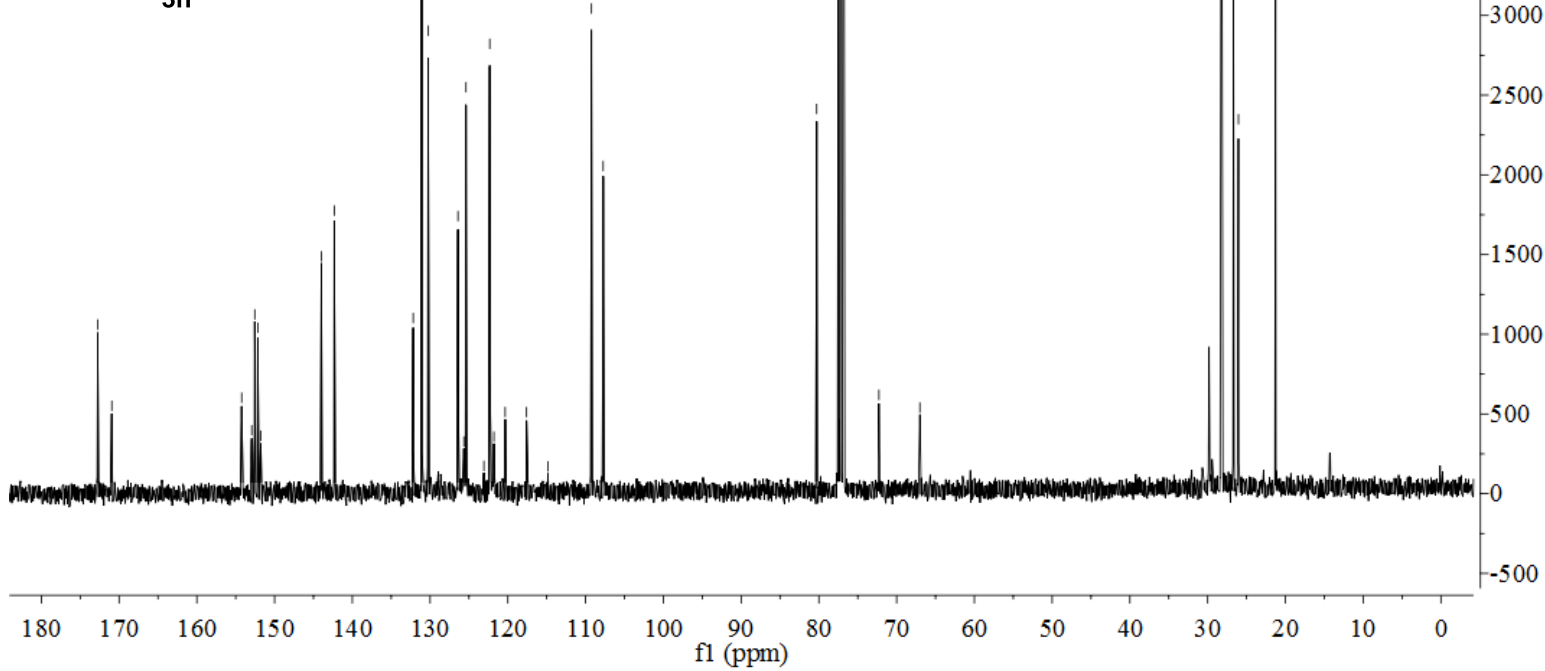

${ }^{19} \mathrm{~F}$ NMR of compound $3 \mathrm{n}\left(376 \mathrm{MHz}\right.$ in $\left.\mathrm{CDCl}_{3}\right)$

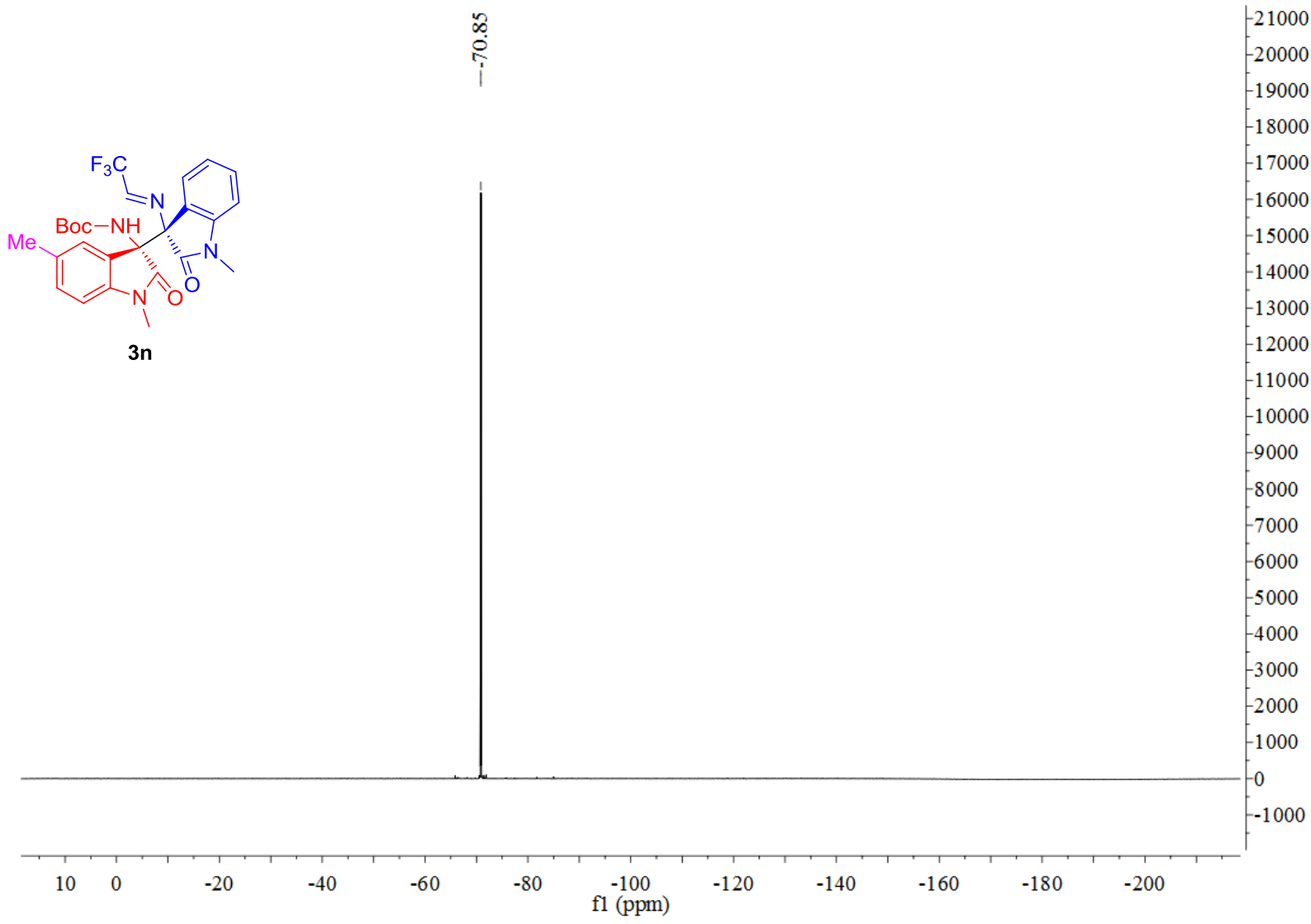


${ }^{1} \mathrm{H}$ NMR of compound $30\left(400 \mathrm{MHz}\right.$ in $\left.\mathrm{CDCl}_{3}\right)$

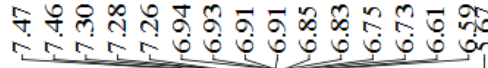

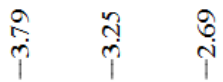

$\stackrel{i}{\mathfrak{4}}$

$-10000$

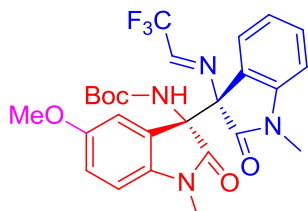

30

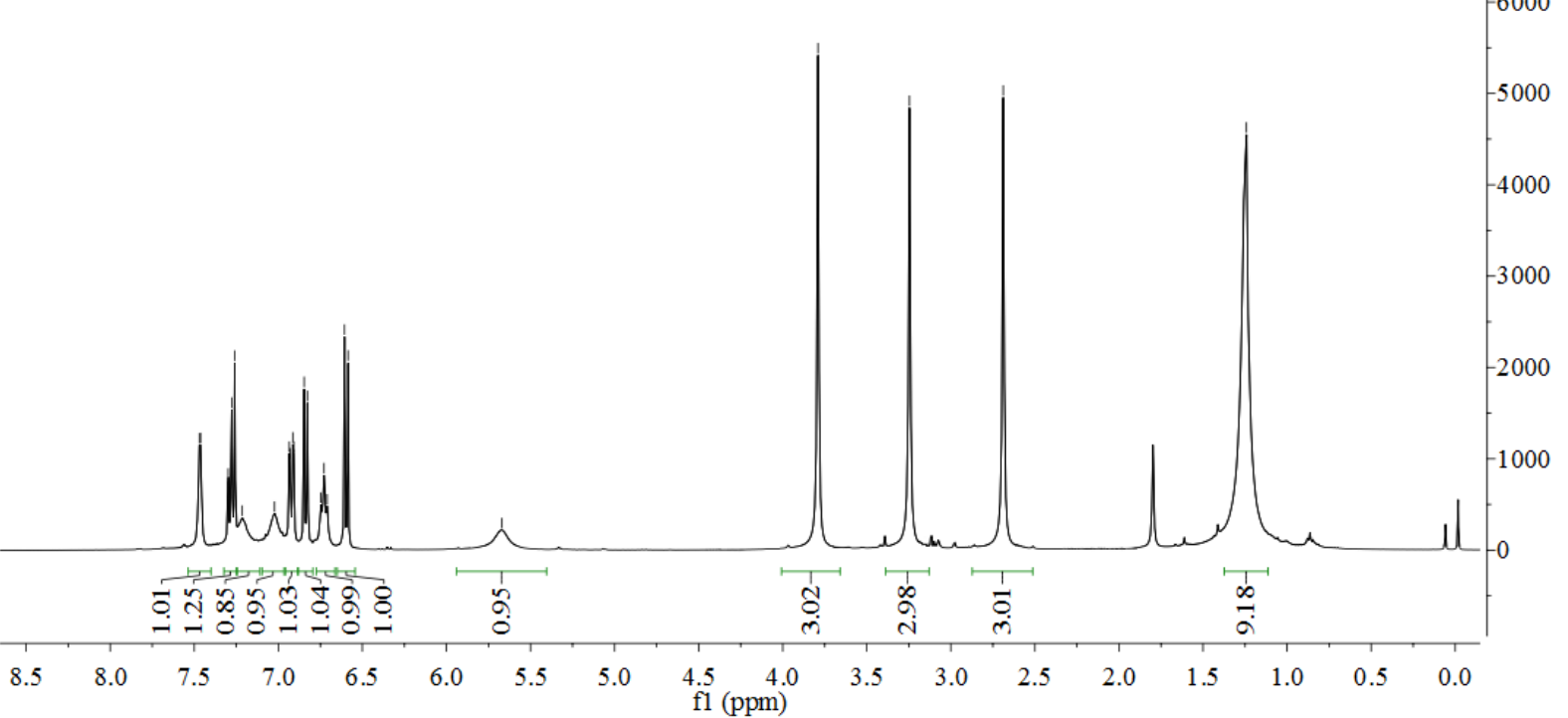

${ }^{13} \mathrm{C}$ NMR of compound 30 (126 $\mathrm{MHz}$ in $\mathrm{CDCl}_{3}$ )

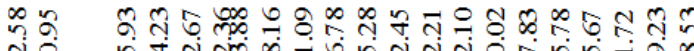

은 约

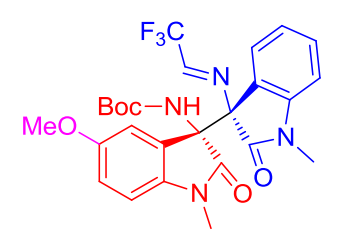

30

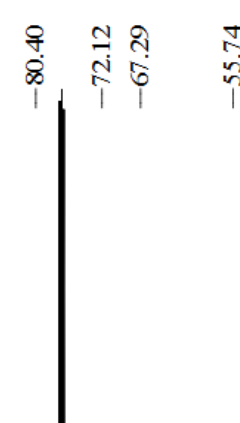

$\begin{array}{ll}8 & 8 \\ 0 & 8 \\ 0 & 0 \\ 0 & 0\end{array}$

3800

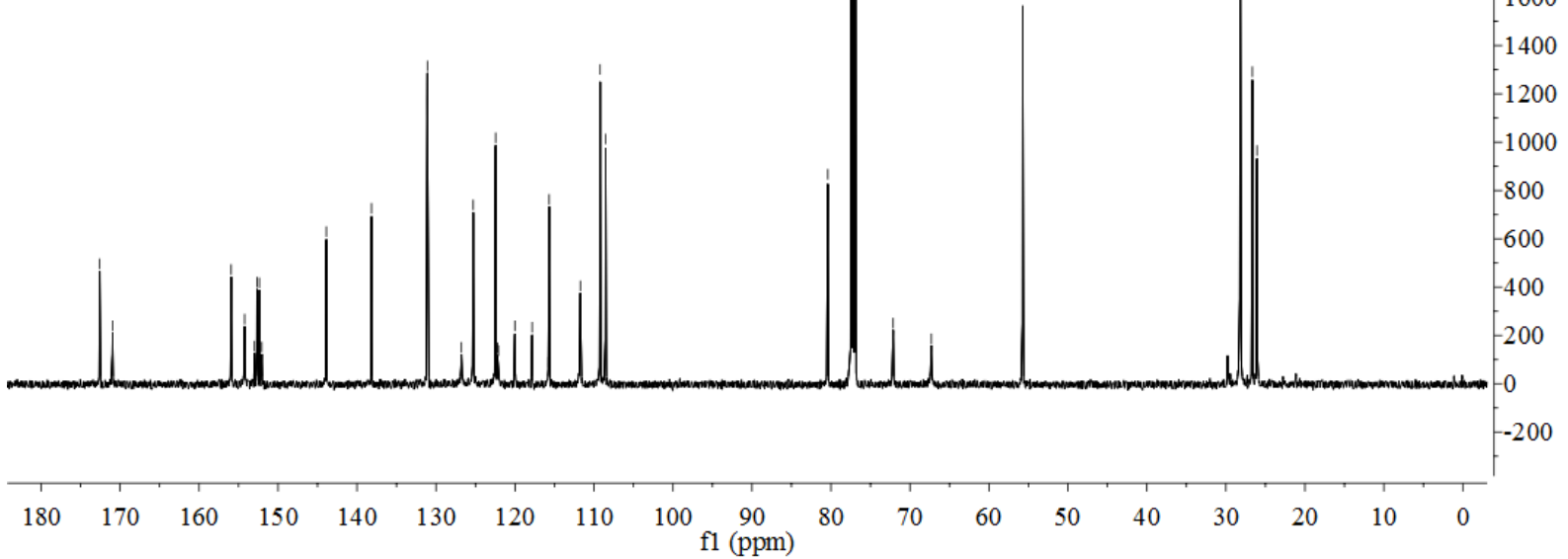


${ }^{19} \mathrm{~F}$ NMR of compound $30\left(376 \mathrm{MHz}\right.$ in $\mathrm{CDCl}_{3}$ )

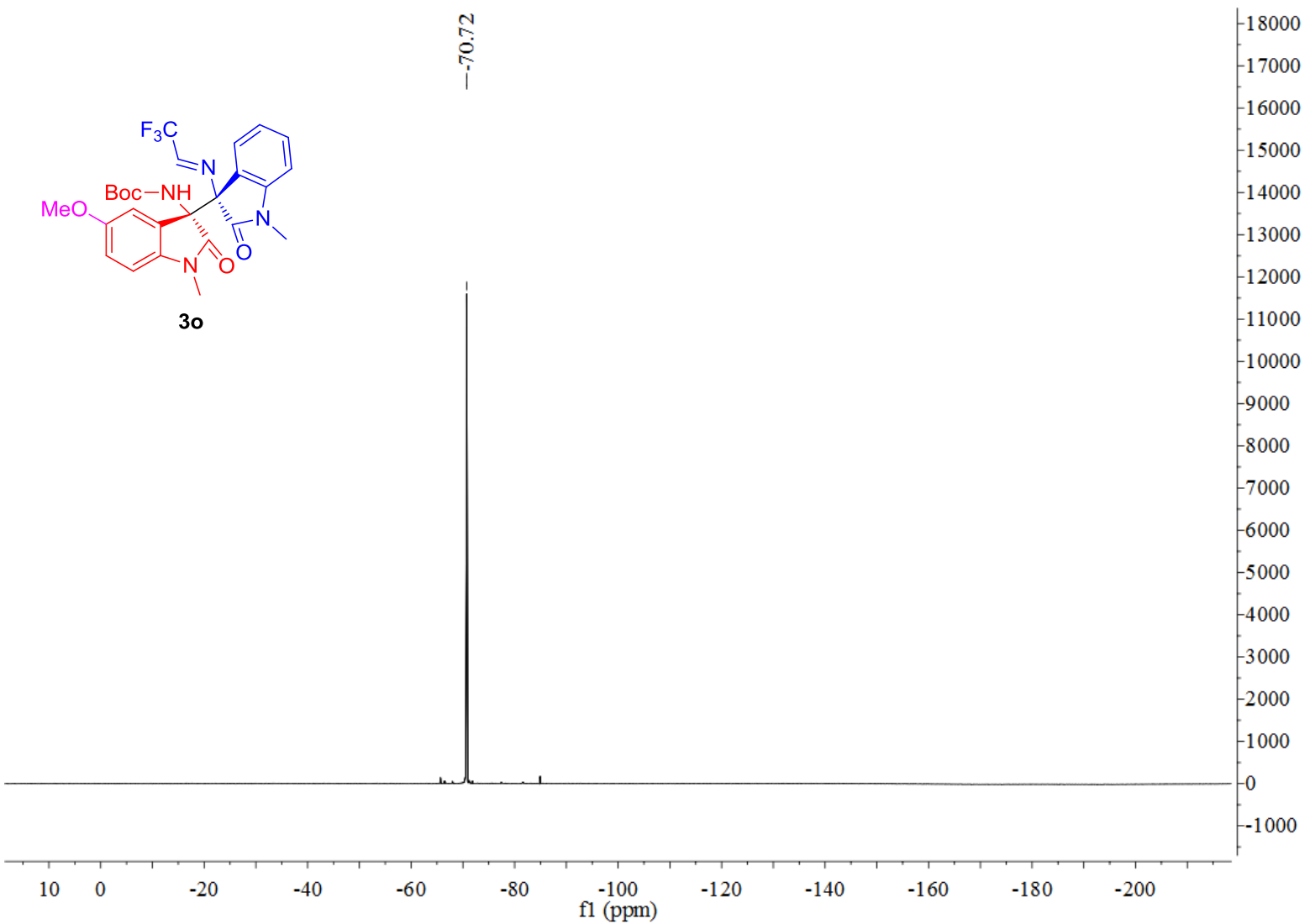

${ }^{1} \mathrm{H}$ NMR of compound $3 \mathrm{p}\left(400 \mathrm{MHz}\right.$ in $\mathrm{CDCl}_{3}$ )

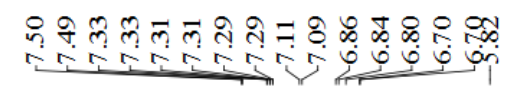

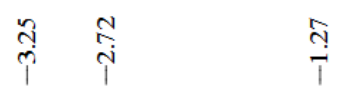

500

000

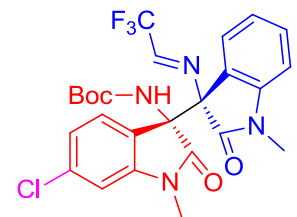

$3 p$

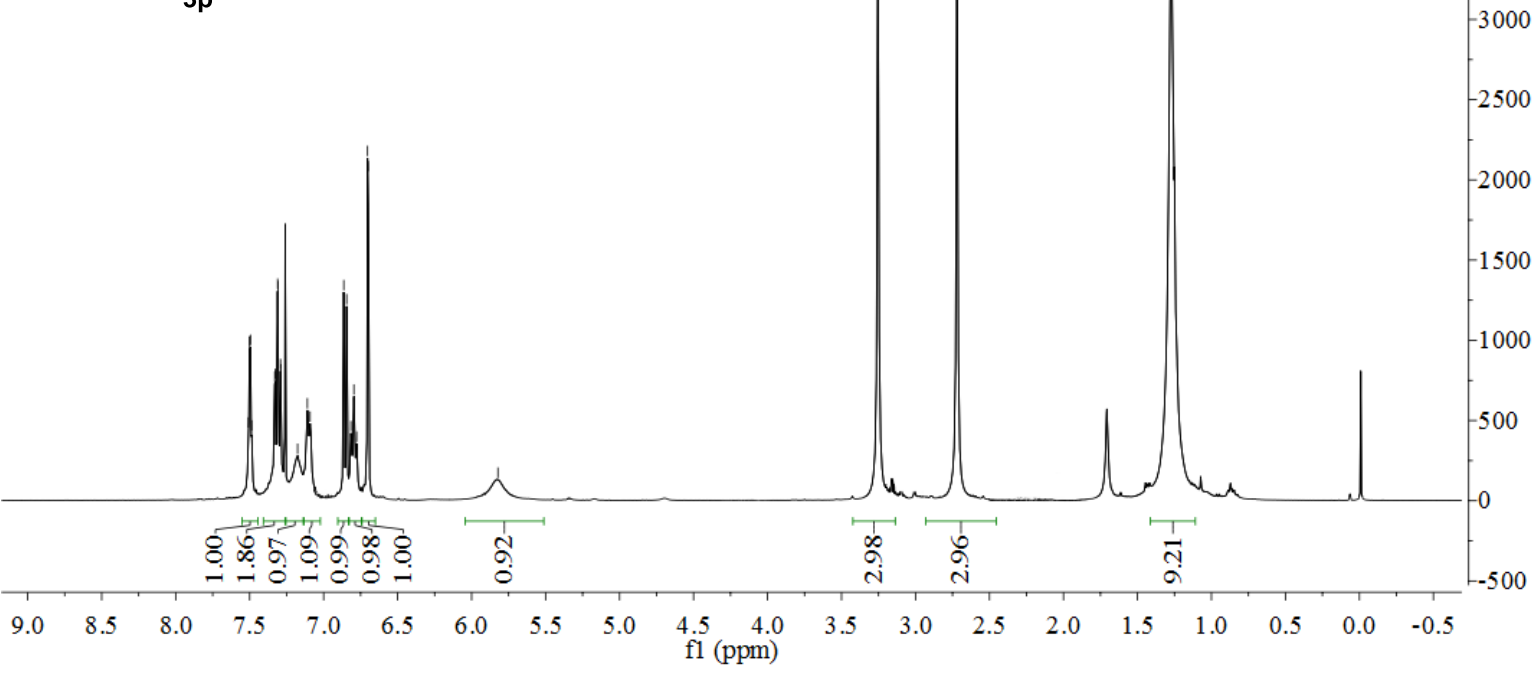


${ }^{13} \mathrm{C}$ NMR of compound $3 p\left(126 \mathrm{MHz}\right.$ in $\mathrm{CDCl}_{3}$ )

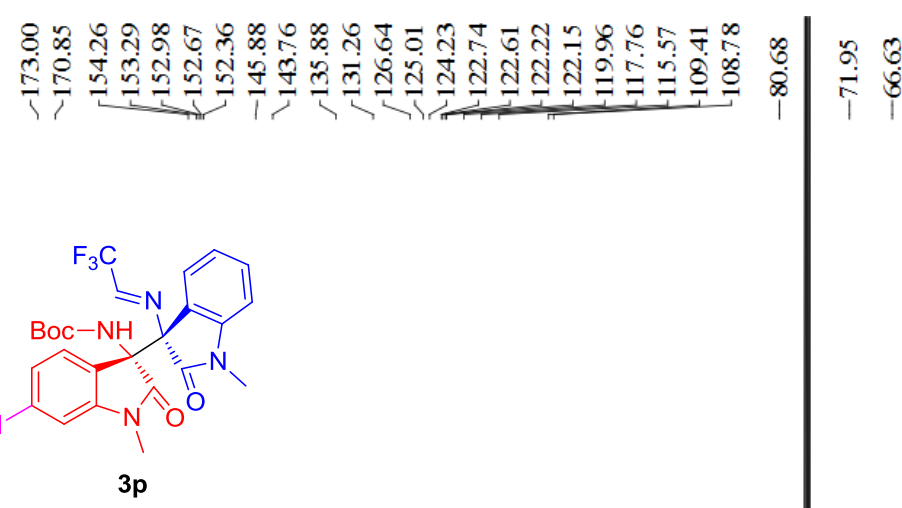

4⿻ำ

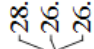

$-2800$

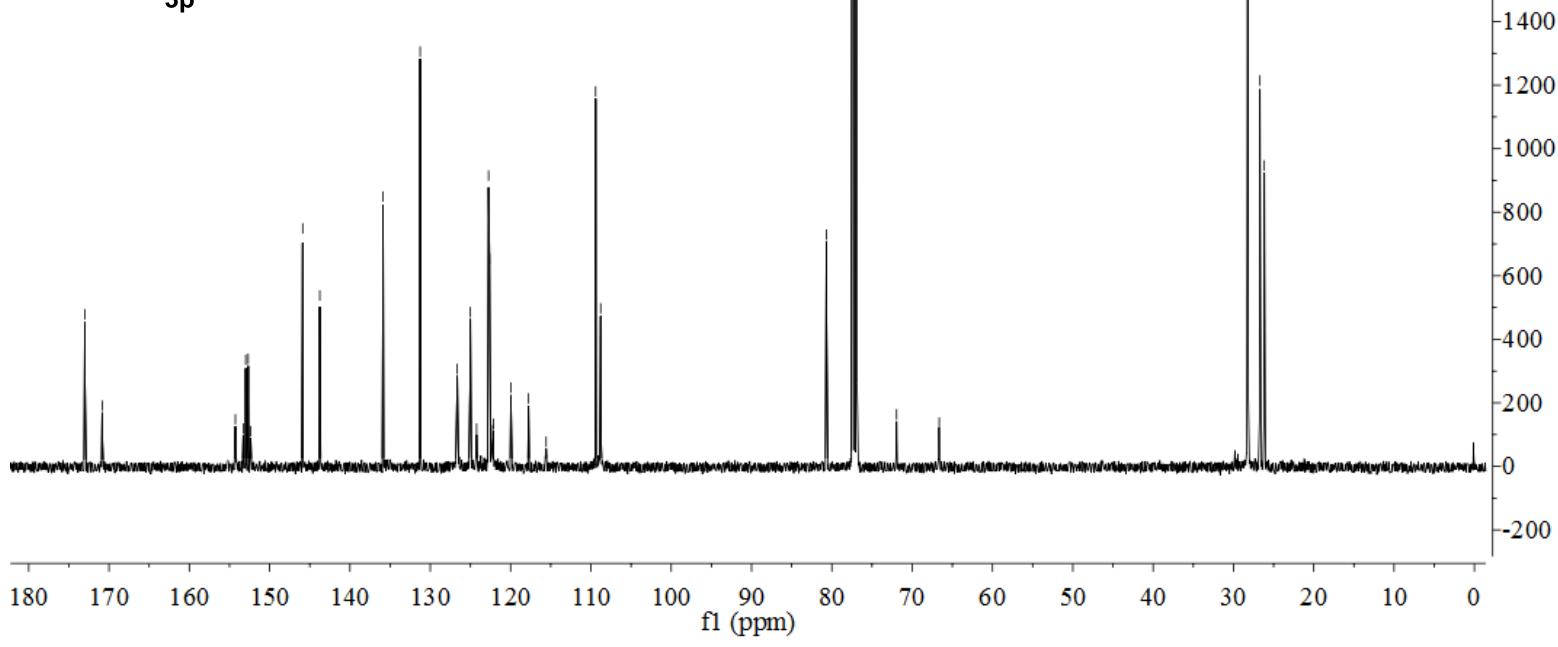

${ }^{19} \mathrm{~F} \mathrm{NMR}$ of compound $3 p\left(376 \mathrm{MHz}\right.$ in $\mathrm{CDCl}_{3}$ )

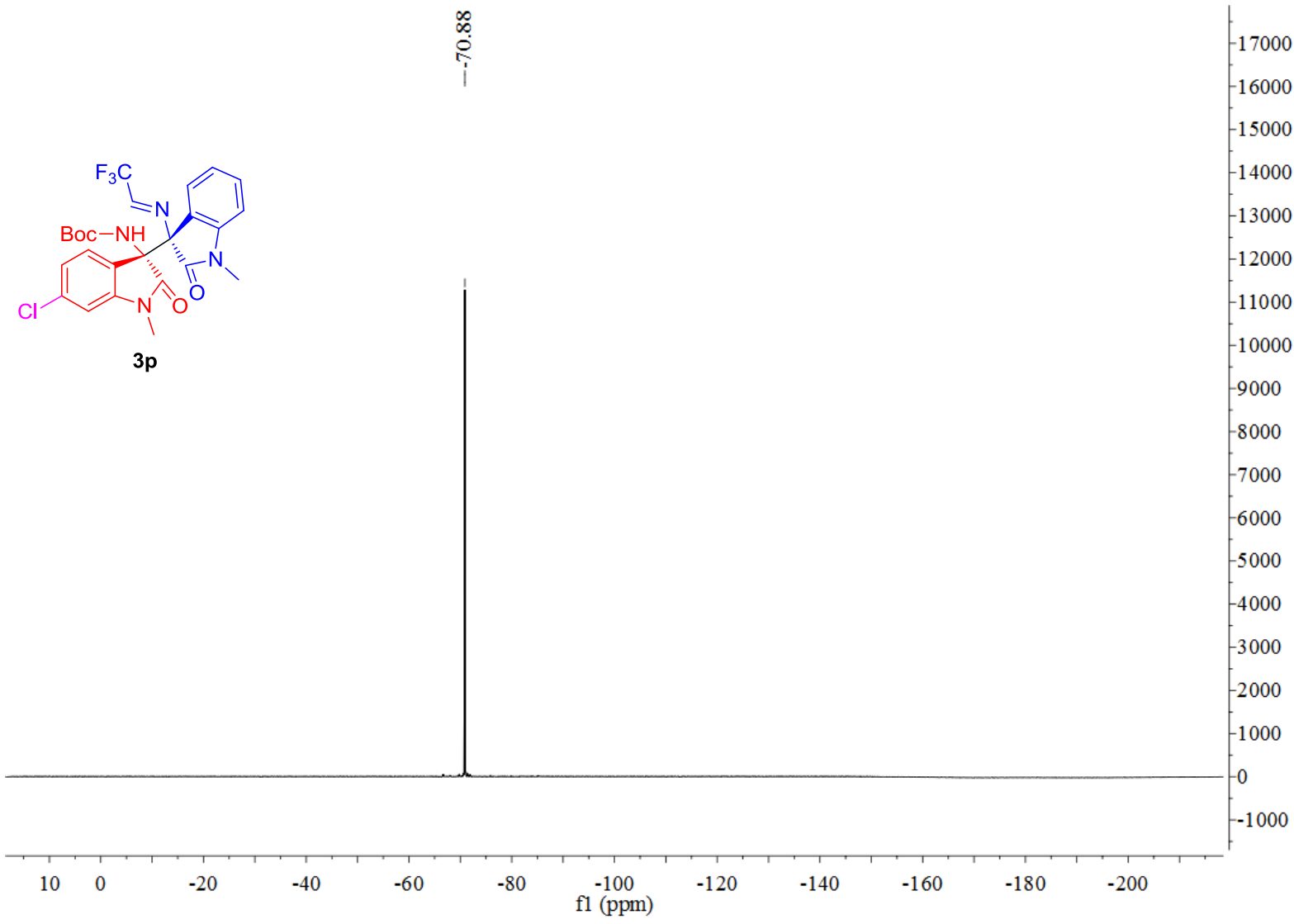


${ }^{1} \mathrm{H}$ NMR of compound $3 q\left(400 \mathrm{MHz}\right.$ in $\mathrm{CDCl}_{3}$ )

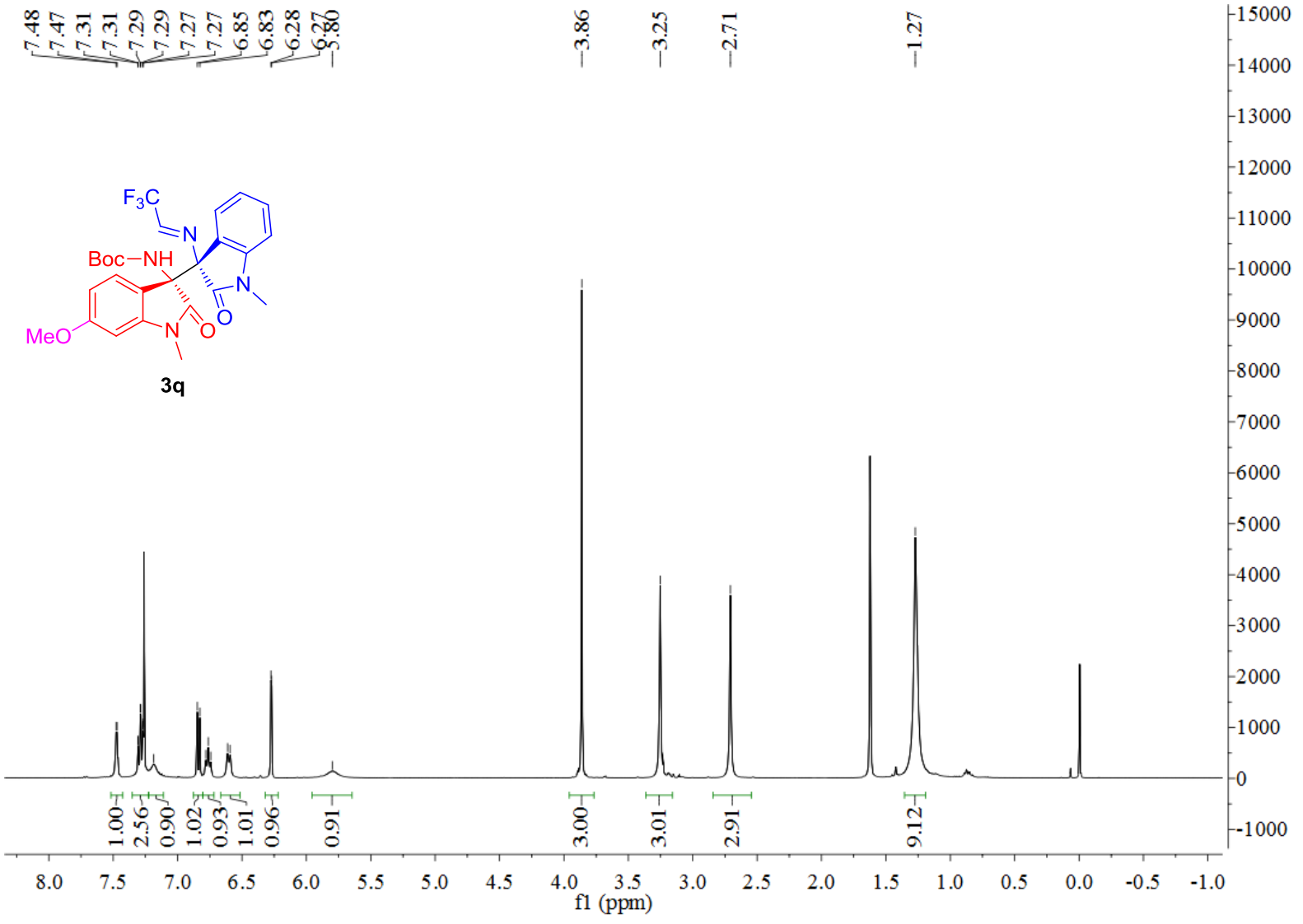

${ }^{13} \mathrm{C}$ NMR of compound $3 q\left(126 \mathrm{MHz}\right.$ in $\mathrm{CDCl}_{3}$ )

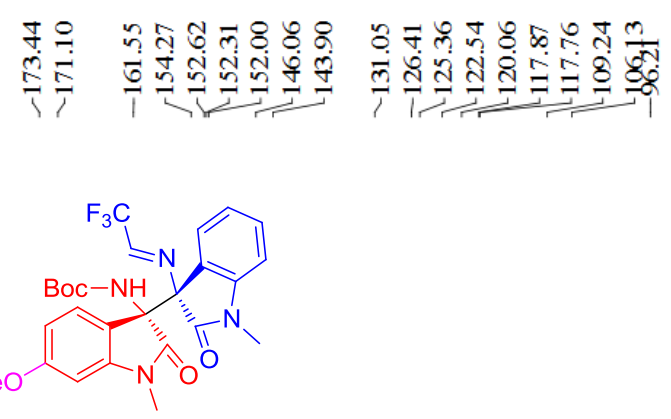

$3 q$

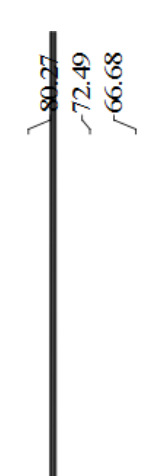

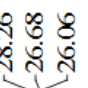

$-1100$

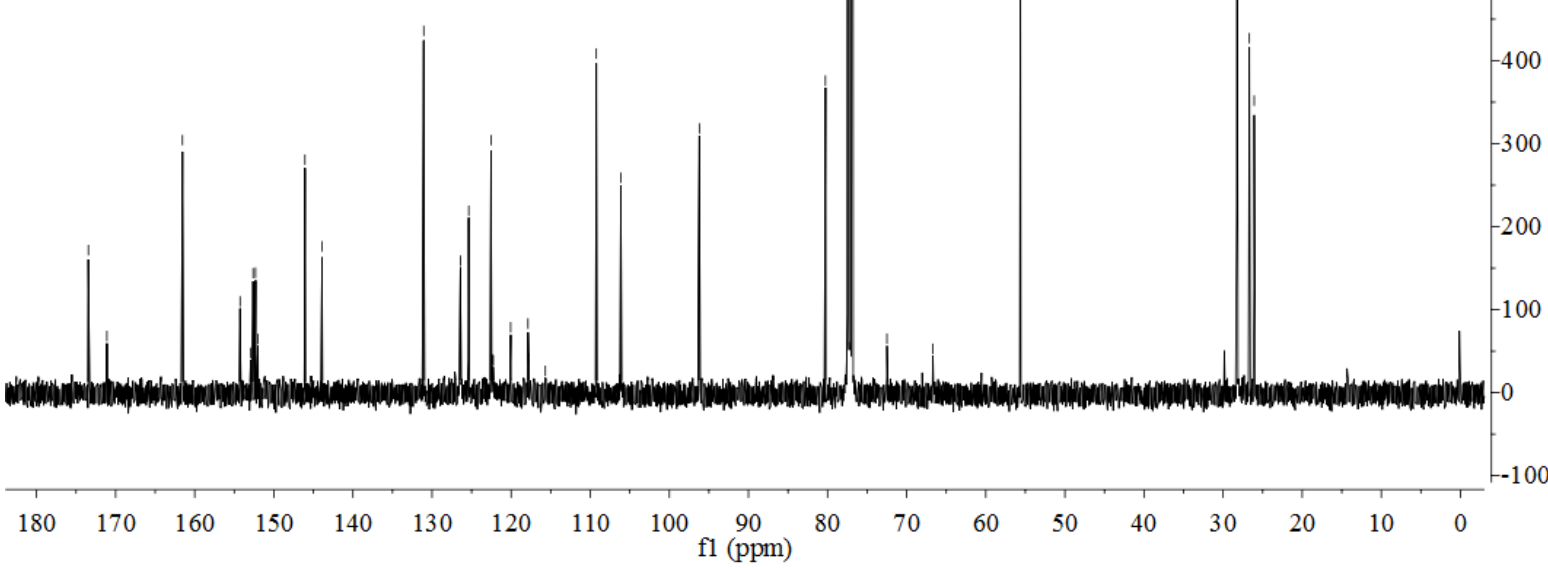


${ }^{19} \mathrm{~F}$ NMR of compound $3 q\left(376 \mathrm{MHz}\right.$ in $\mathrm{CDCl}_{3}$ )

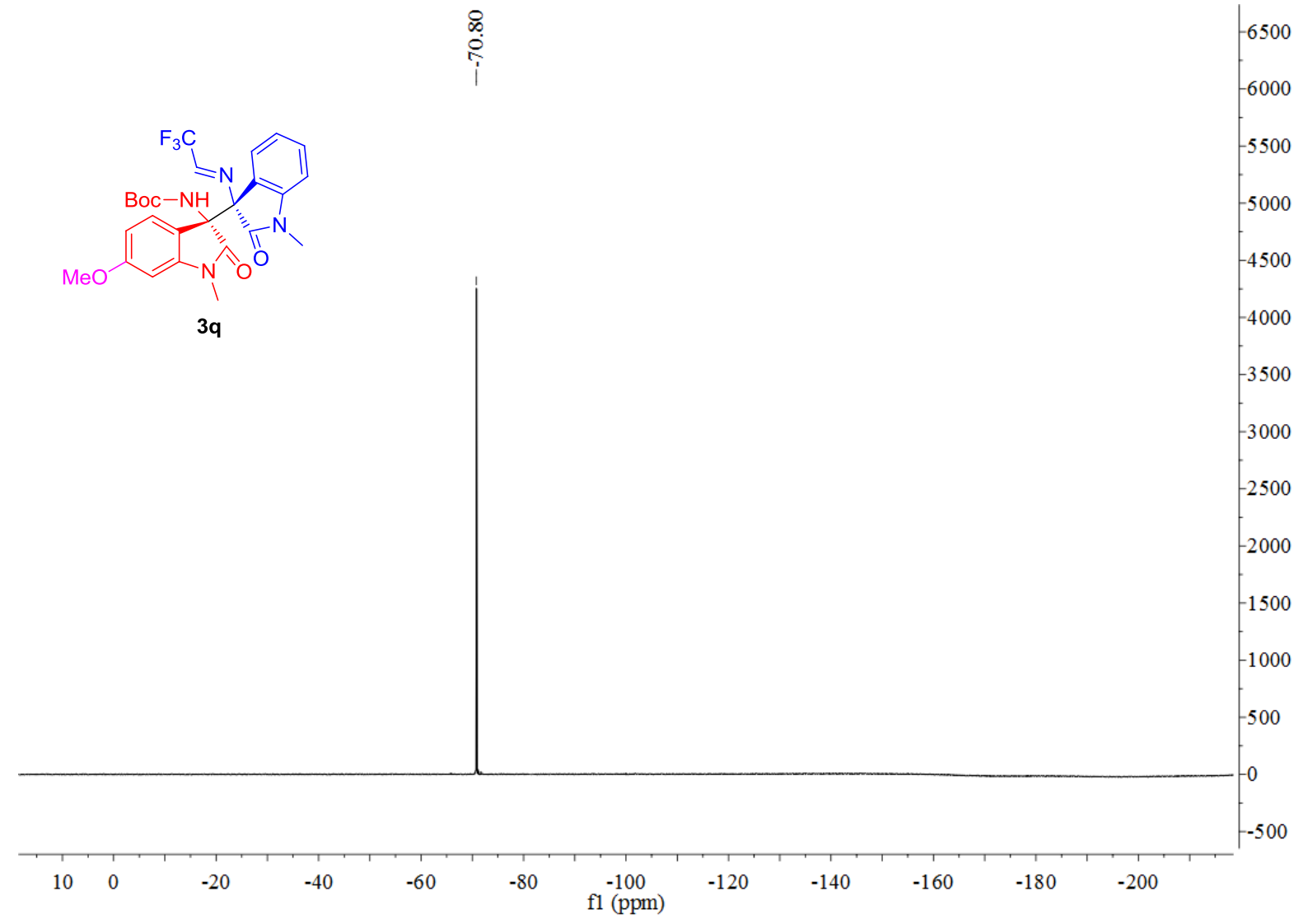

${ }^{1} \mathrm{H}$ NMR of compound $3 \mathrm{r}\left(400 \mathrm{MHz}\right.$ in $\left.\mathrm{CDCl}_{3}\right)$

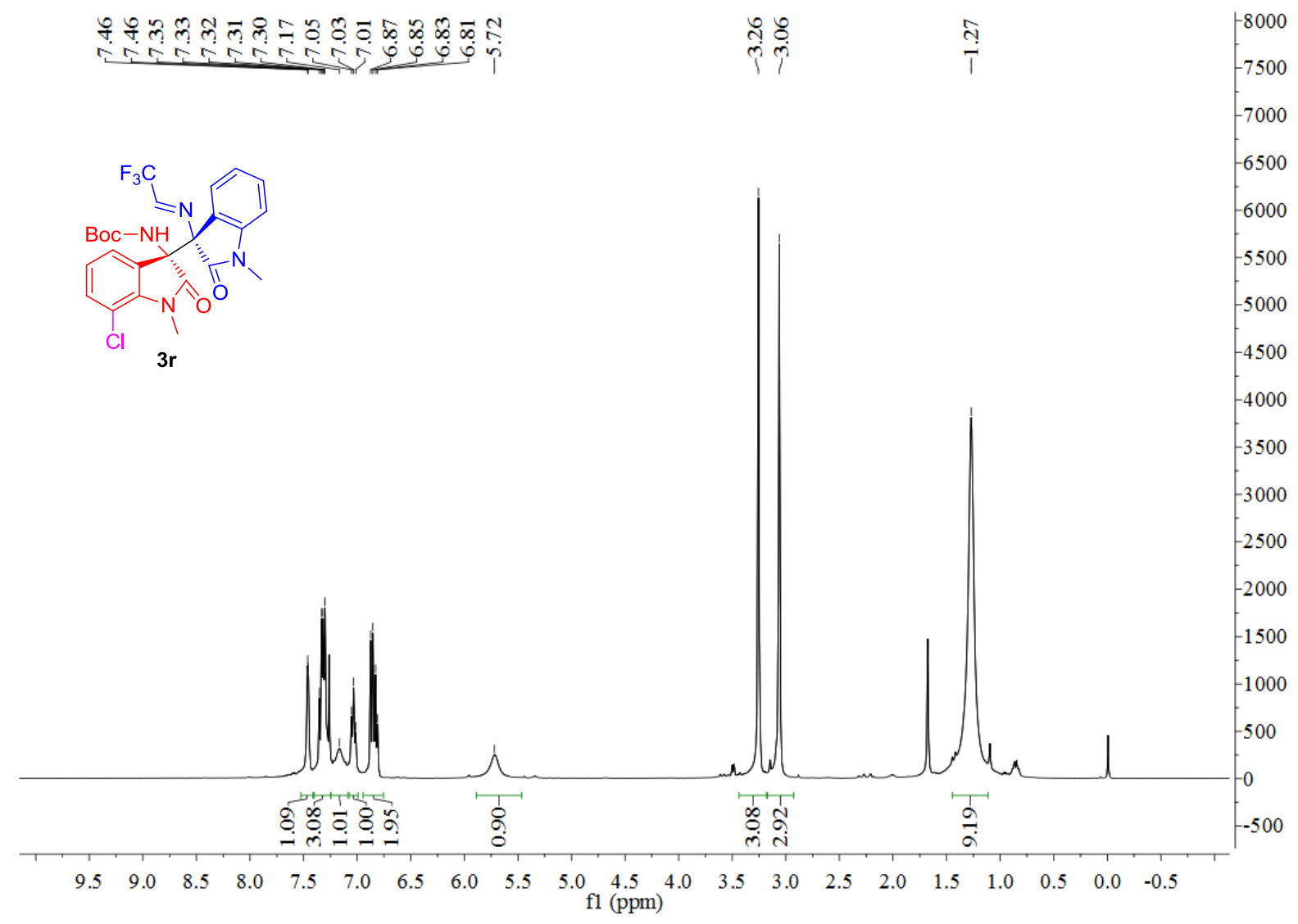


${ }^{13} \mathrm{C}$ NMR of compound $3 \mathrm{r}\left(101 \mathrm{MHz}\right.$ in $\left.\mathrm{CDCl}_{3}\right)$

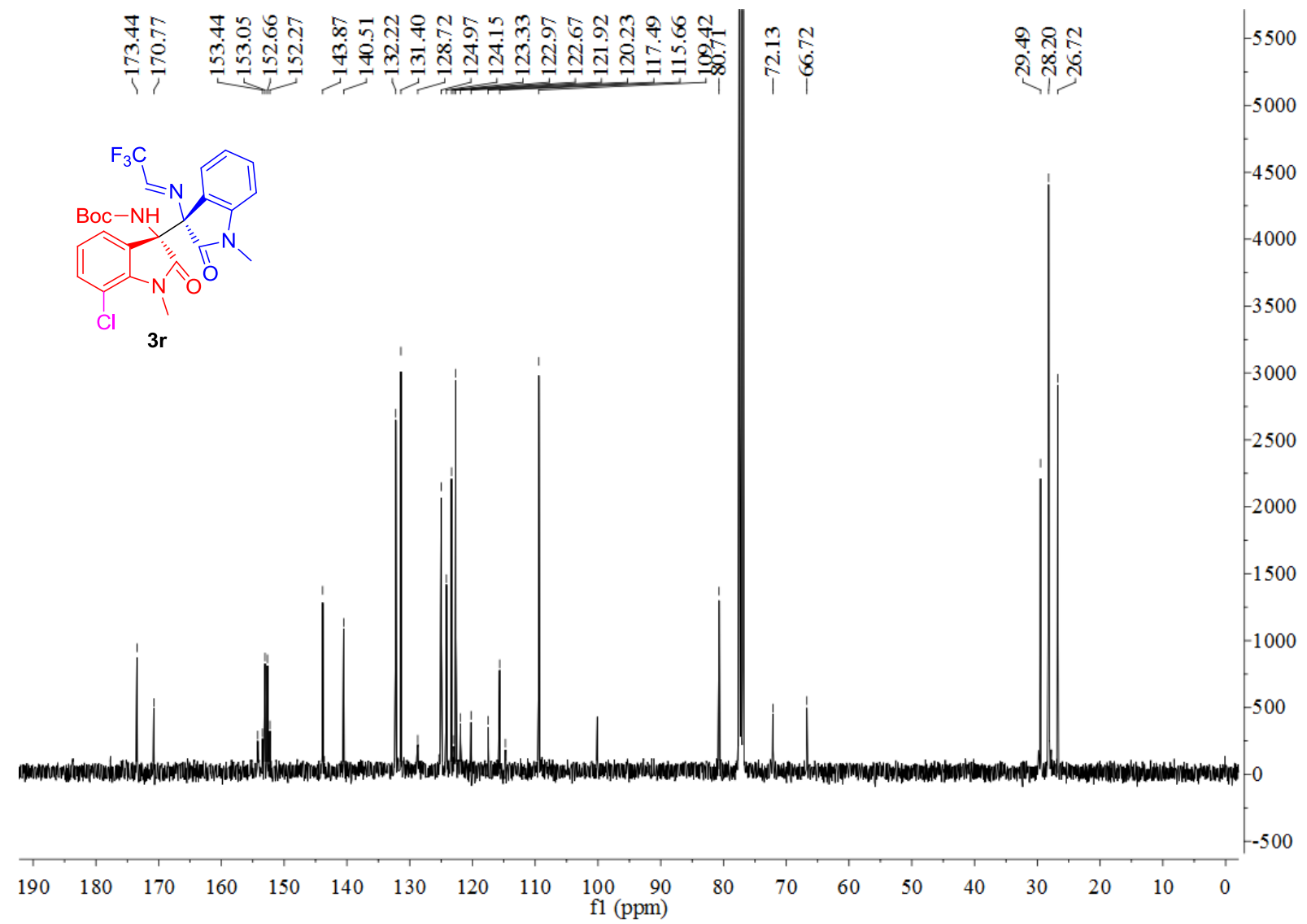

${ }^{19} \mathrm{~F}$ NMR of compound $3 \mathrm{r}\left(376 \mathrm{MHz}\right.$ in $\mathrm{CDCl}_{3}$ )

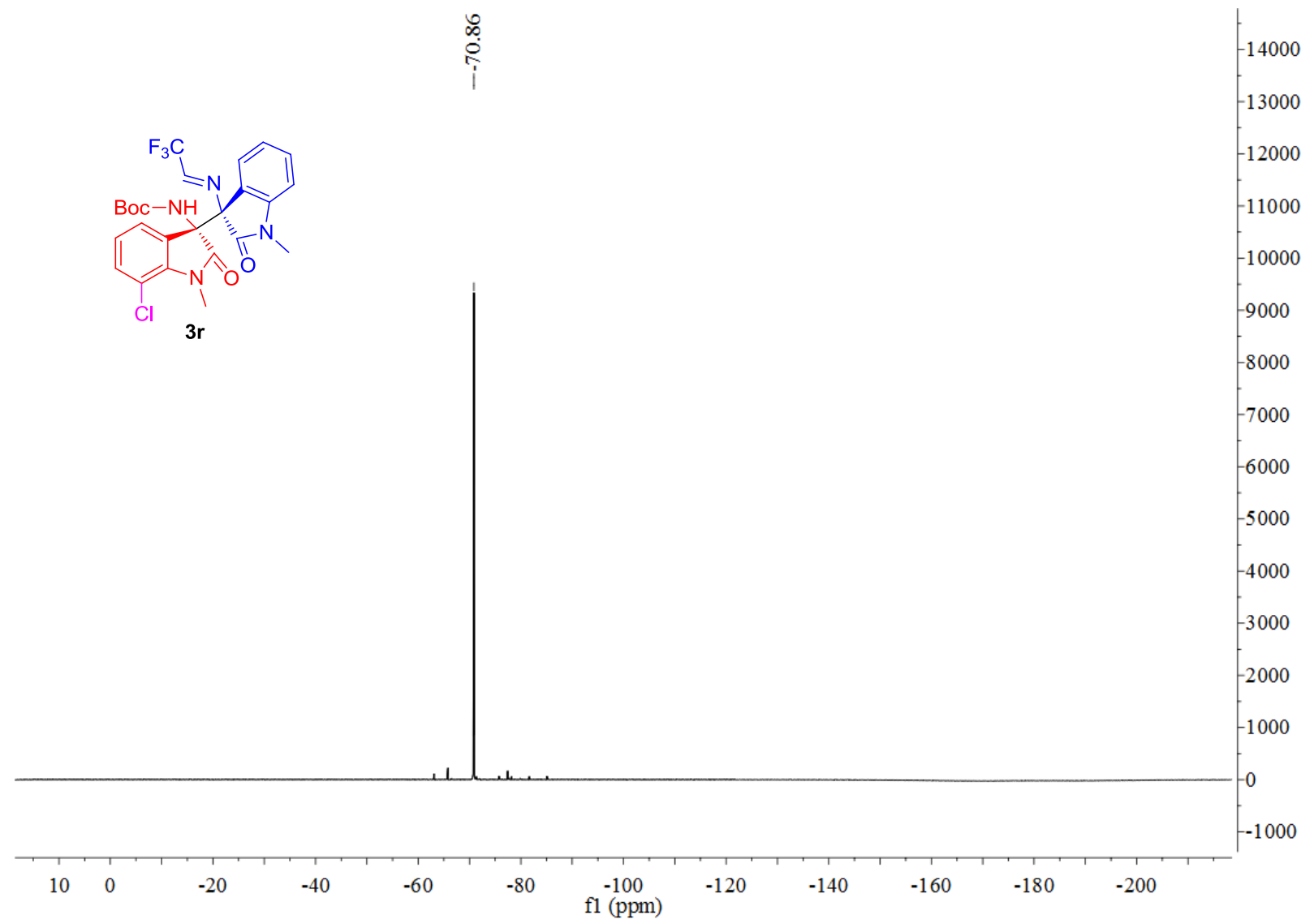


${ }^{1} \mathrm{H}$ NMR of compound $3 \mathrm{~s}\left(400 \mathrm{MHz}\right.$ in $\left.\mathrm{CDCl}_{3}\right)$

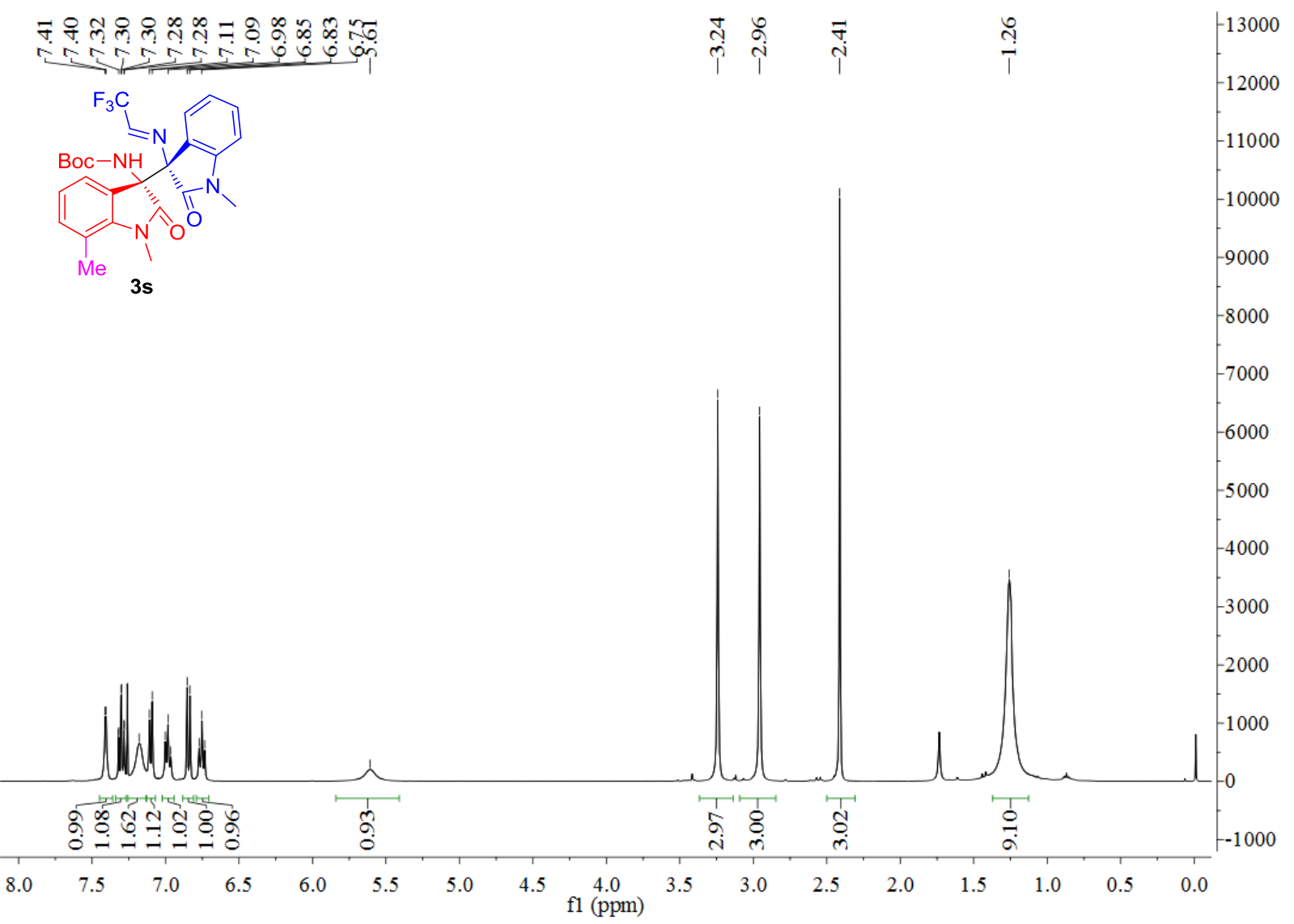

${ }^{13} \mathrm{C}$ NMR of compound 3s (101 $\mathrm{MHz}$ in $\mathrm{CDCl}_{3}$ )

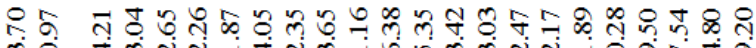

mo

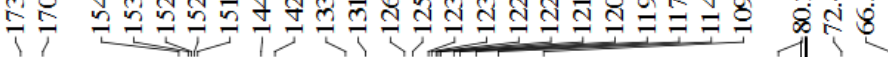
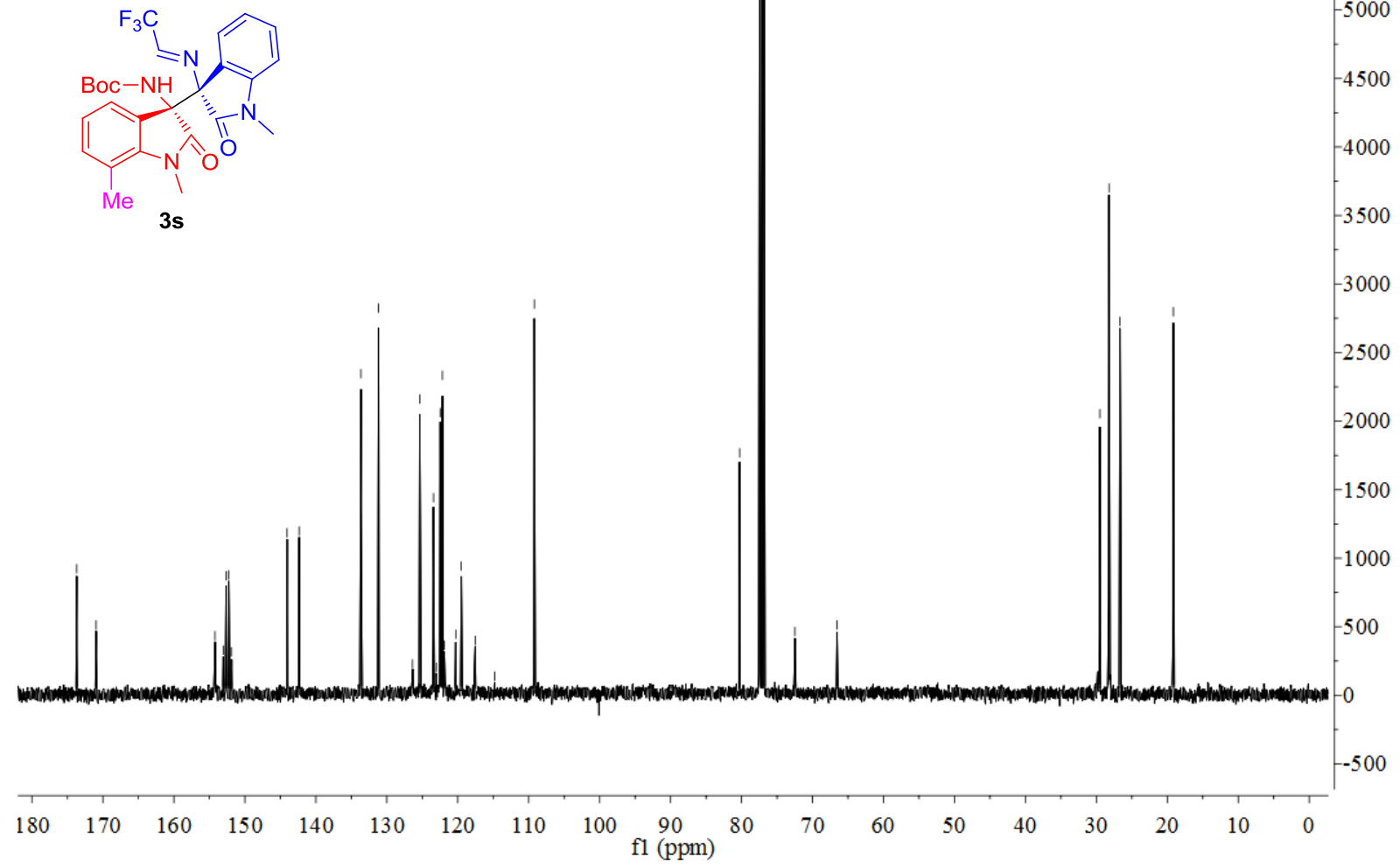
${ }^{19} \mathrm{~F} \mathrm{NMR}$ of compound 3s (376 $\mathrm{MHz}$ in $\mathrm{CDCl}_{3}$ )

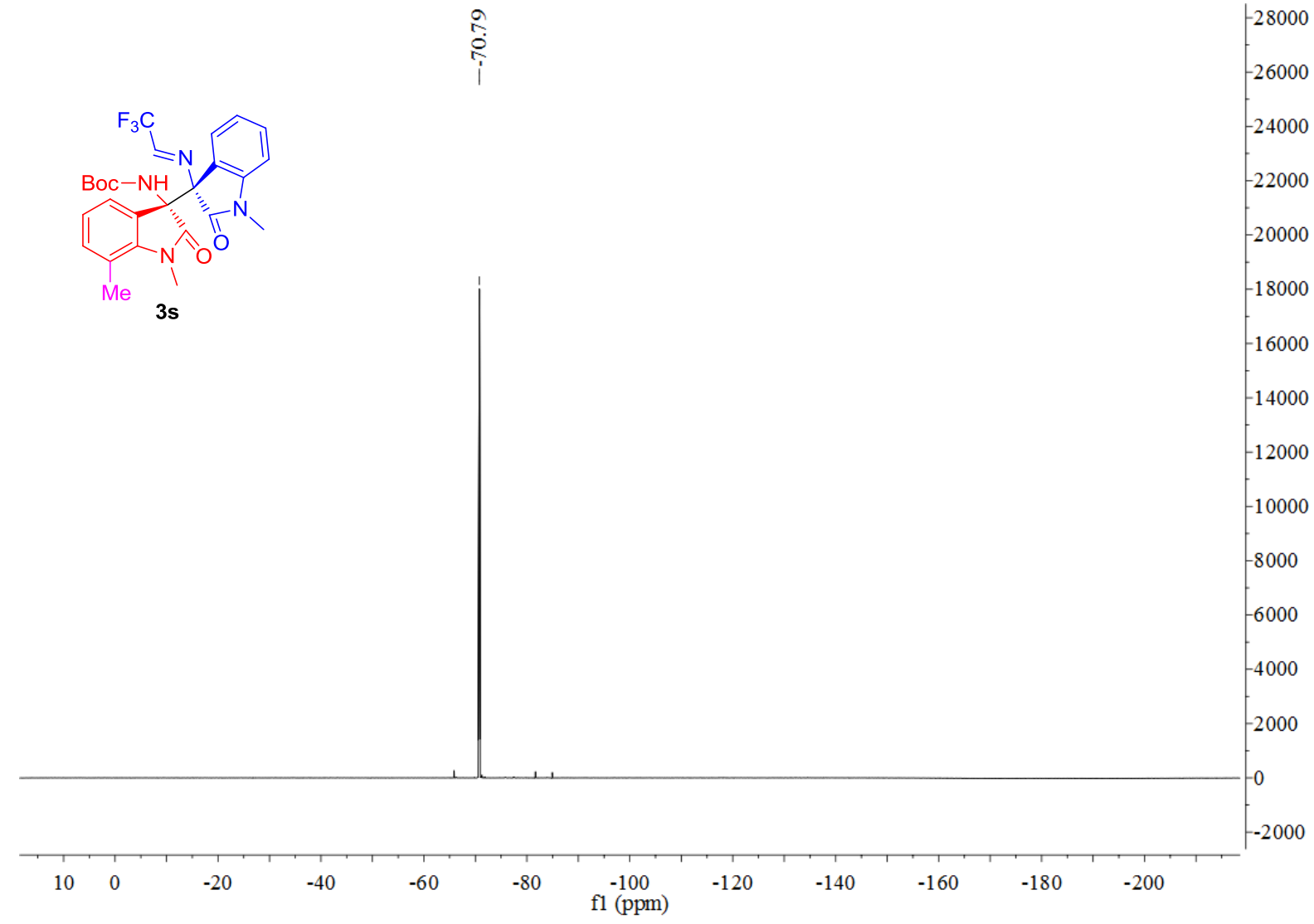

${ }^{1} \mathrm{H}$ NMR of compound $3 \mathrm{t}$ ( $400 \mathrm{MHz}$ in $\mathrm{CDCl}_{3}$ )

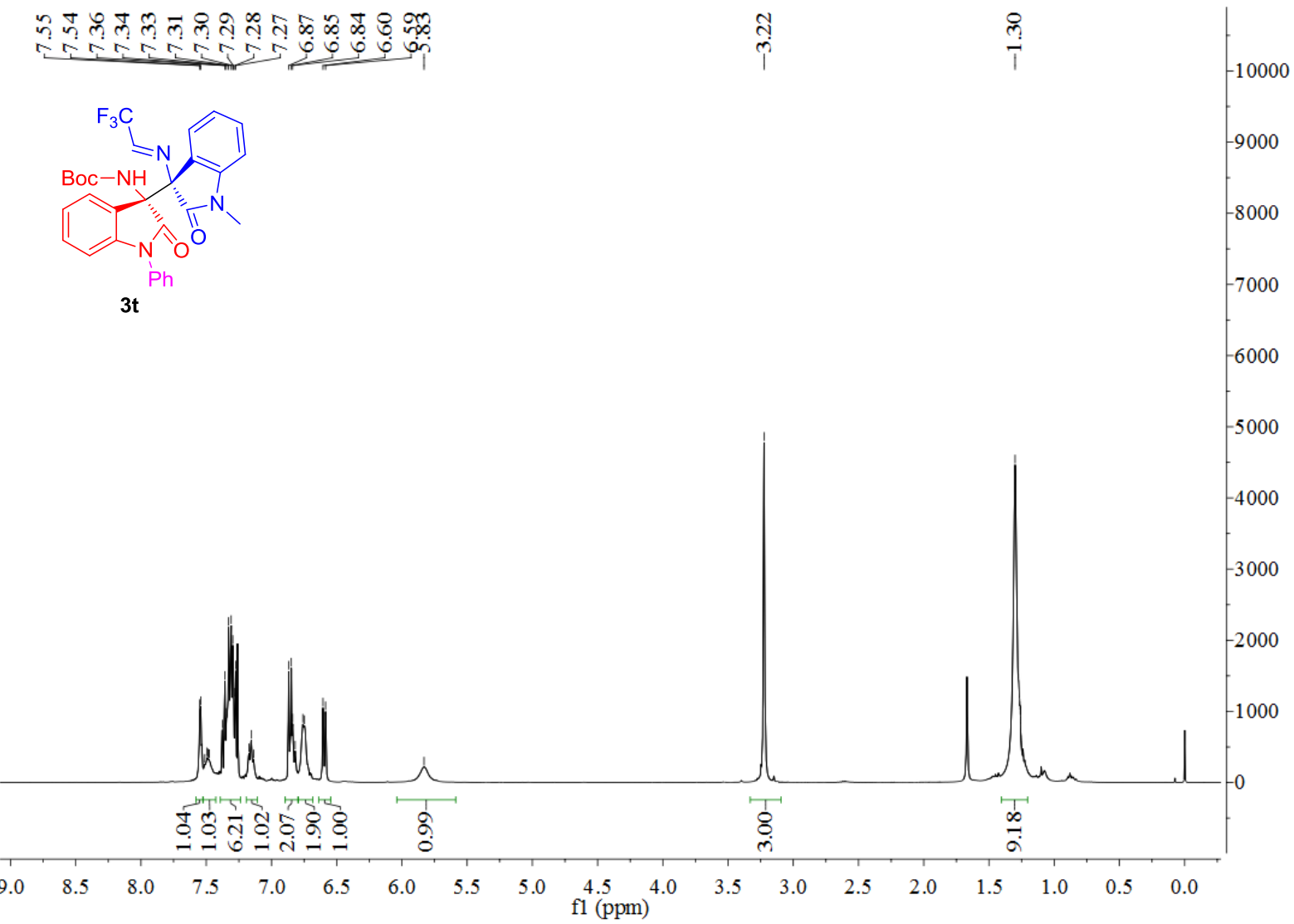


${ }^{13} \mathrm{C}$ NMR of compound 3t (101 $\mathrm{MHz}$ in $\mathrm{CDCl}_{3}$ )

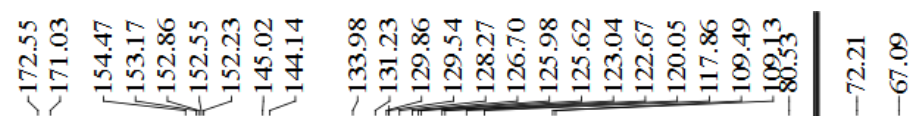

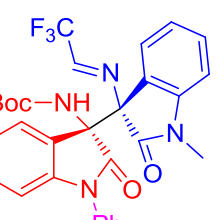

3t

$\mathrm{Ph}$

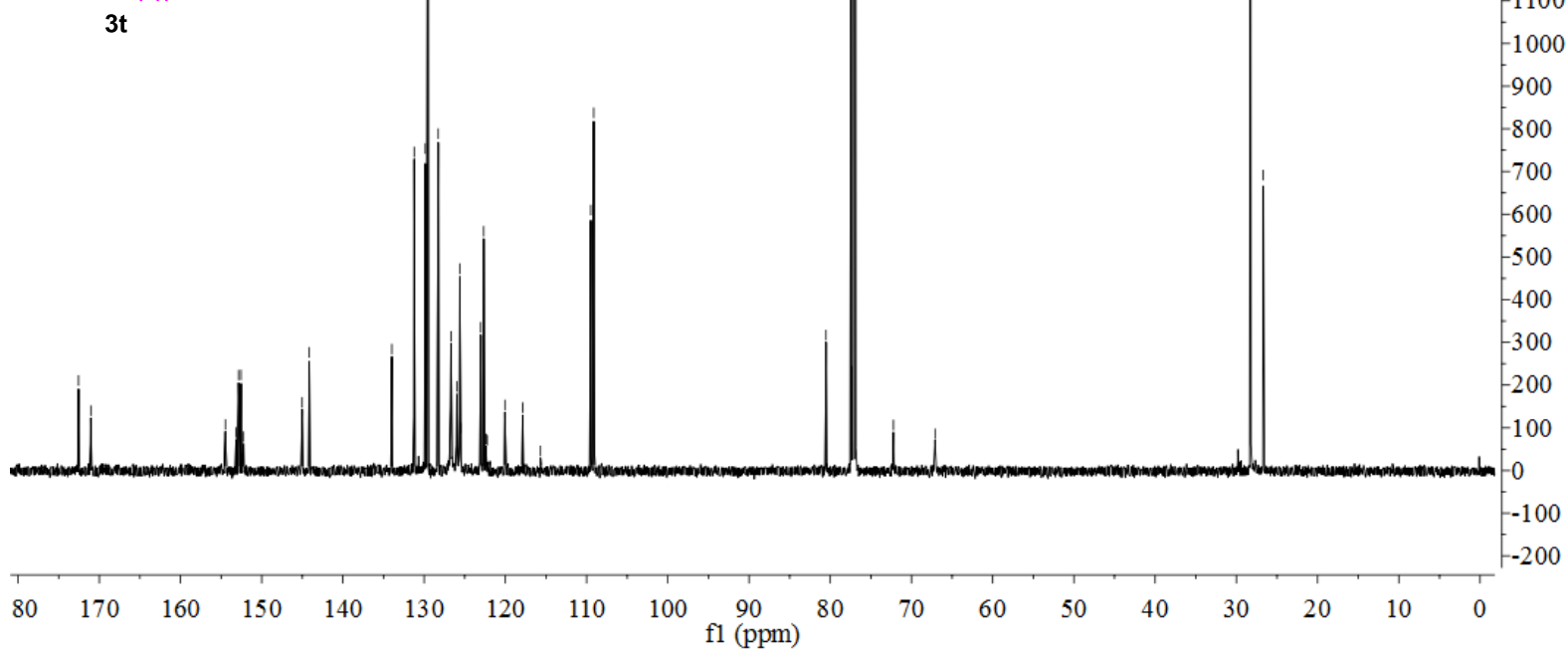

${ }^{19} \mathrm{~F}$ NMR of compound $3 t\left(376 \mathrm{MHz}\right.$ in $\left.\mathrm{CDCl}_{3}\right)$

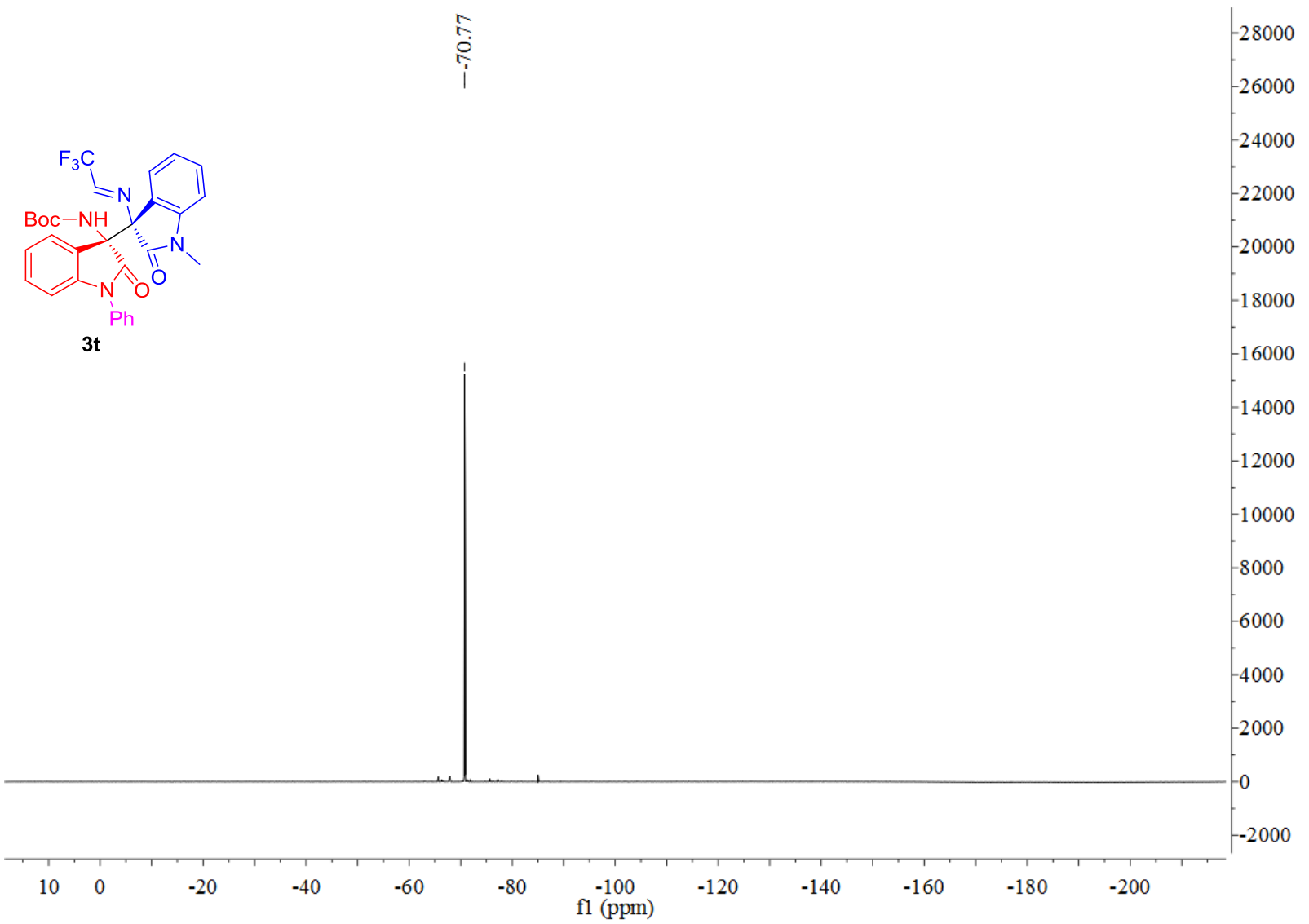




\section{${ }^{1} \mathrm{H}$ NMR of compound $3 \mathrm{u}\left(400 \mathrm{MHz}\right.$ in $\left.\mathrm{CDCl}_{3}\right)$}

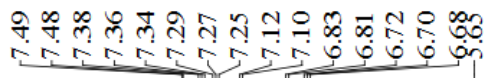

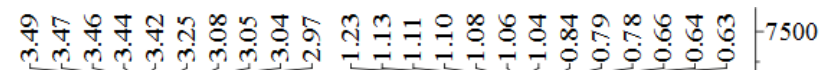

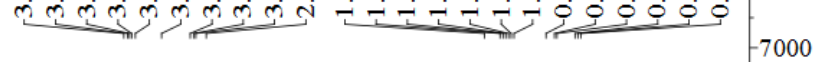

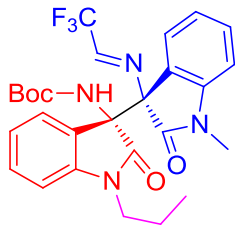

$3 \mathbf{u}$

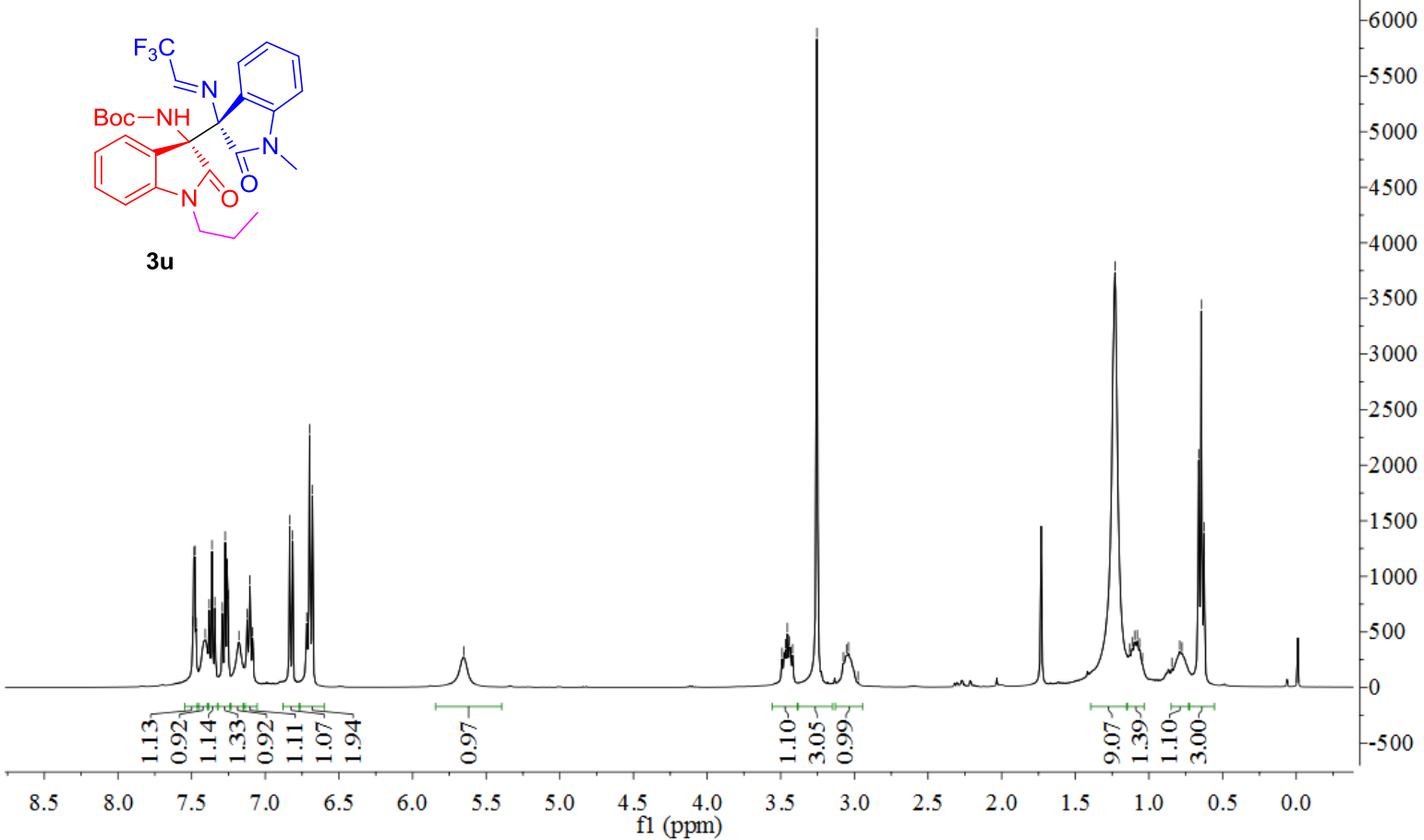

${ }^{13} \mathrm{C}$ NMR of compound $3 \mathrm{u}\left(101 \mathrm{MHz}\right.$ in $\mathrm{CDCl}_{3}$ )

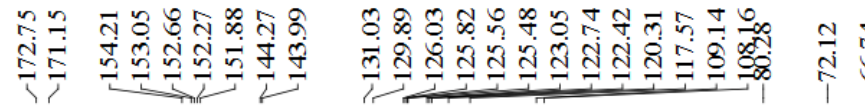

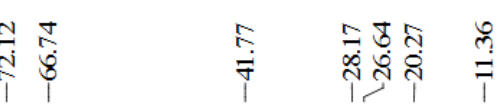

7000

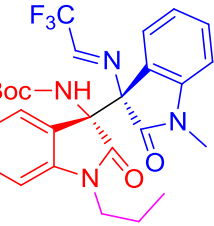

$3 \mathbf{u}$

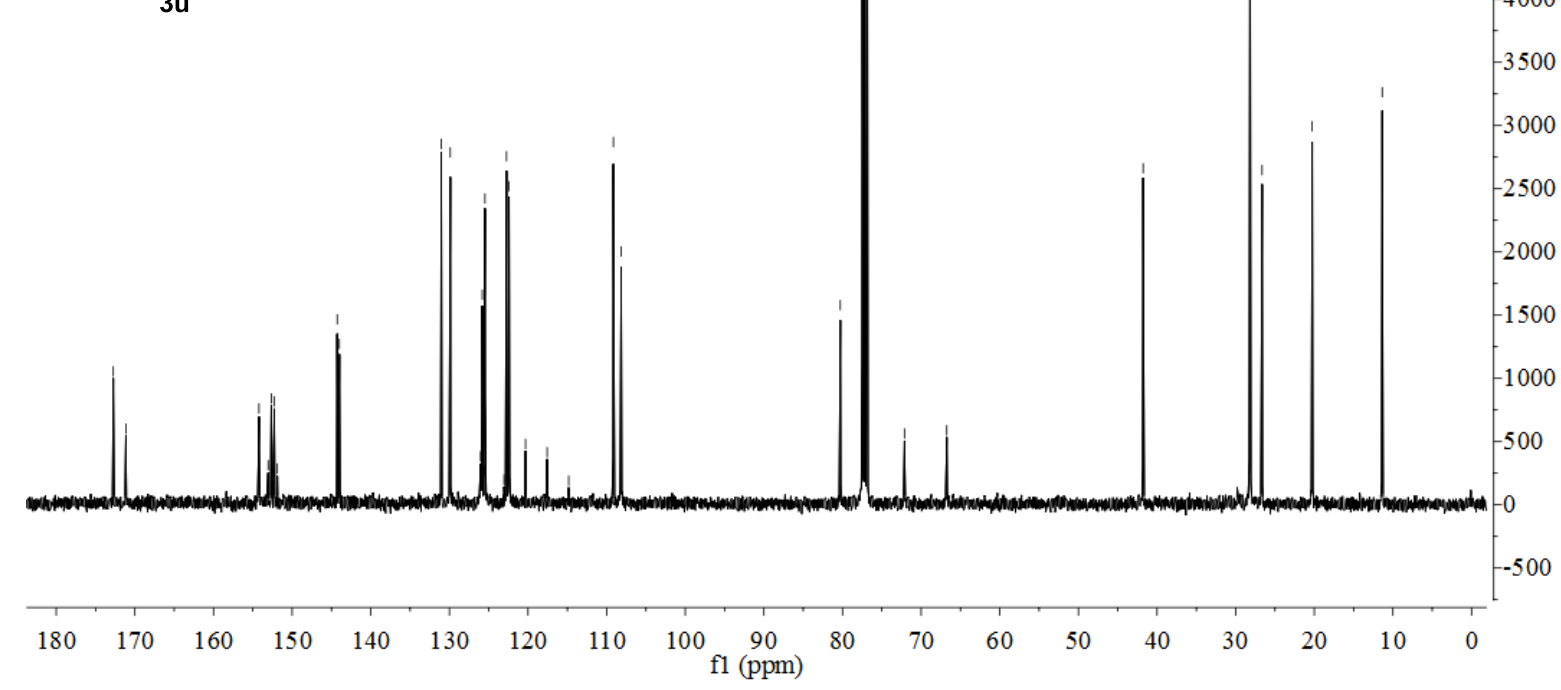


${ }^{19} \mathrm{~F}$ NMR of compound $3 \mathrm{u}\left(376 \mathrm{MHz}\right.$ in $\mathrm{CDCl}_{3}$ )

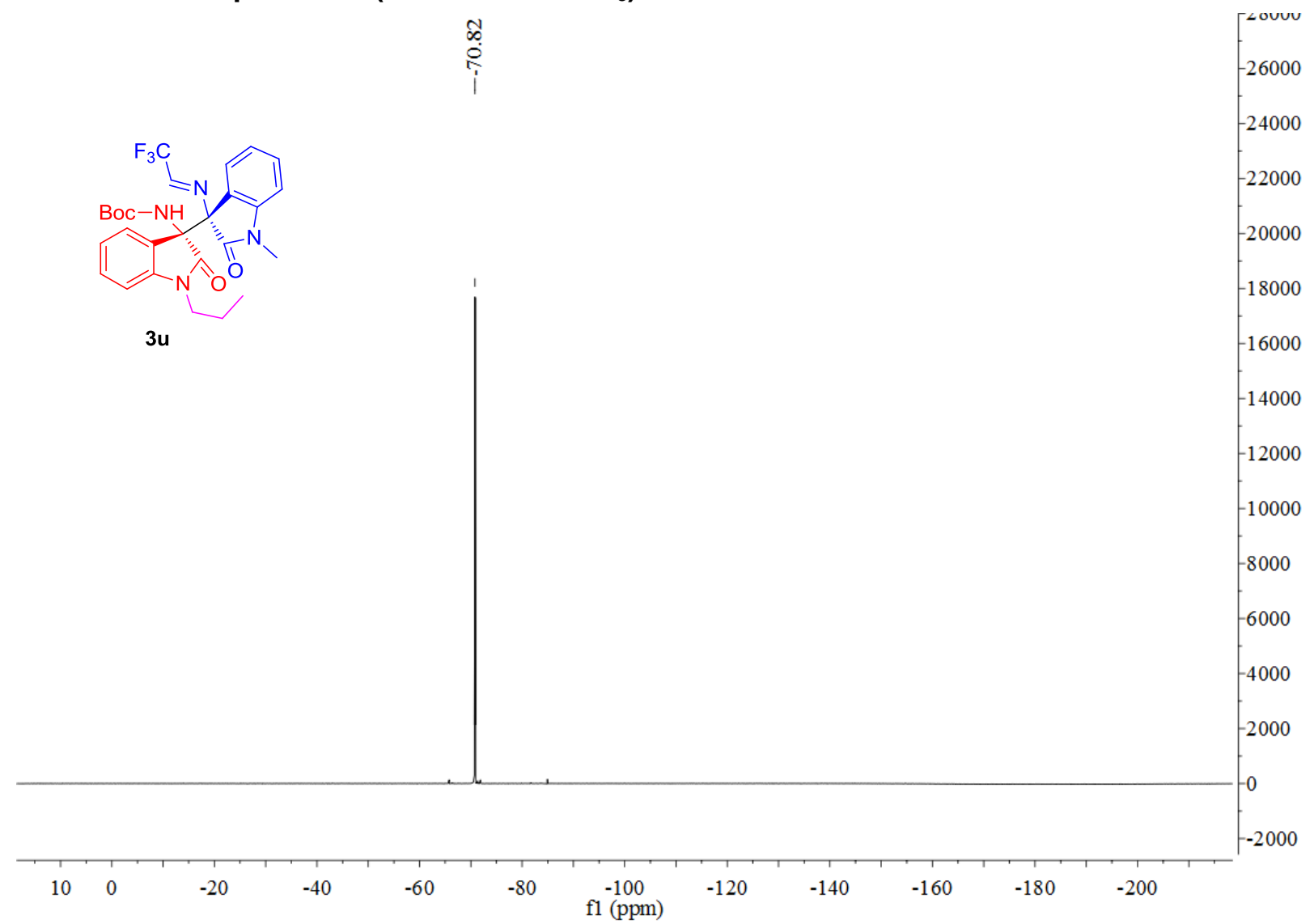

${ }^{1} \mathrm{H}$ NMR of compound $3 \mathrm{v}\left(400 \mathrm{MHz}\right.$ in $\left.\mathrm{CDCl}_{3}\right)$

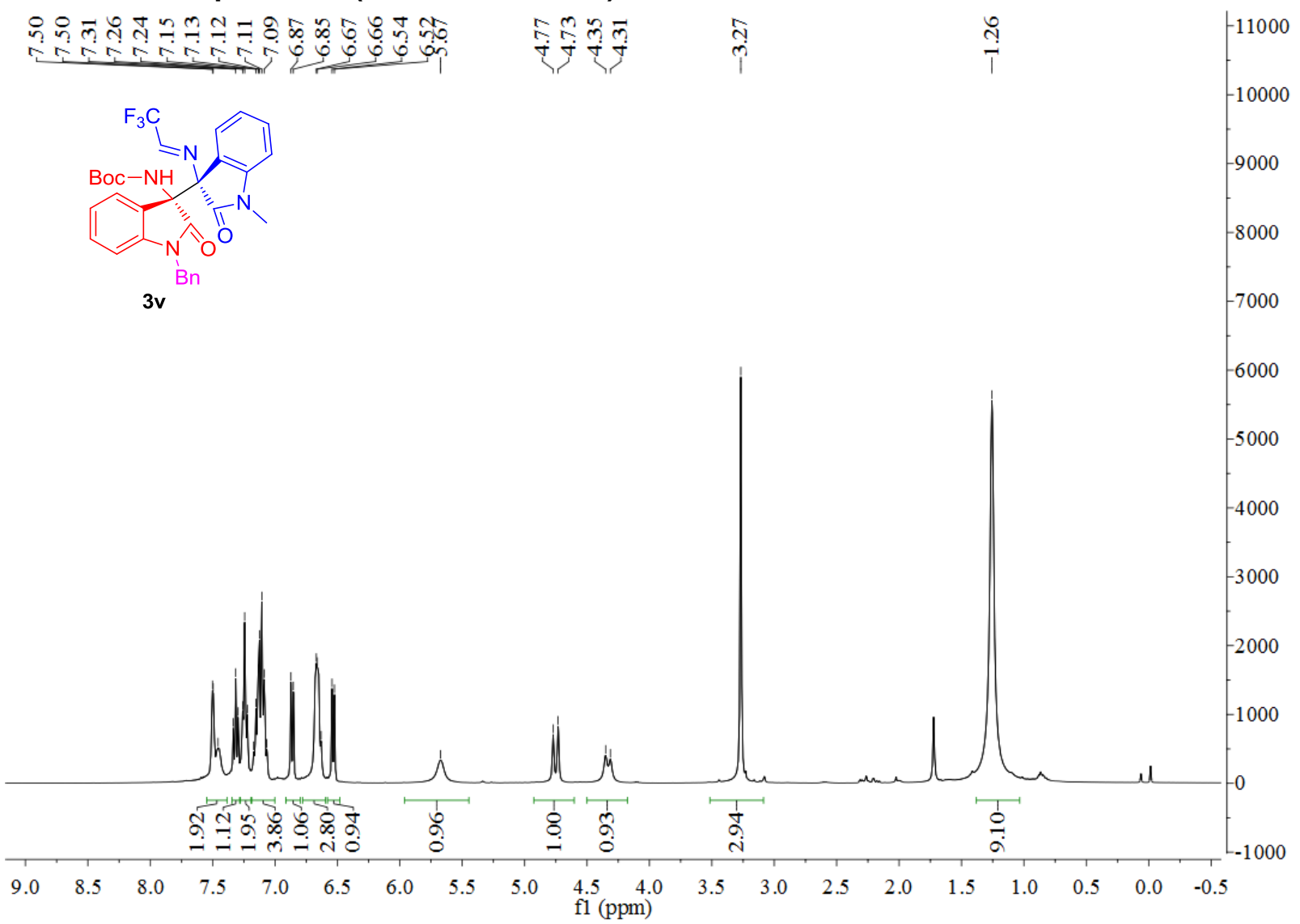


${ }^{13} \mathrm{C}$ NMR of compound $3 \mathrm{v}\left(101 \mathrm{MHz}\right.$ in $\mathrm{CDCl}_{3}$ )

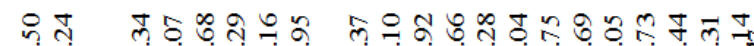

I)

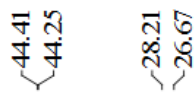

15000

4000

3000

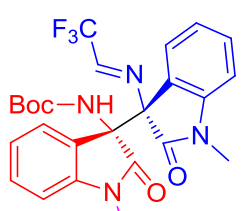

$3 \mathbf{v}^{\mathrm{B}}$

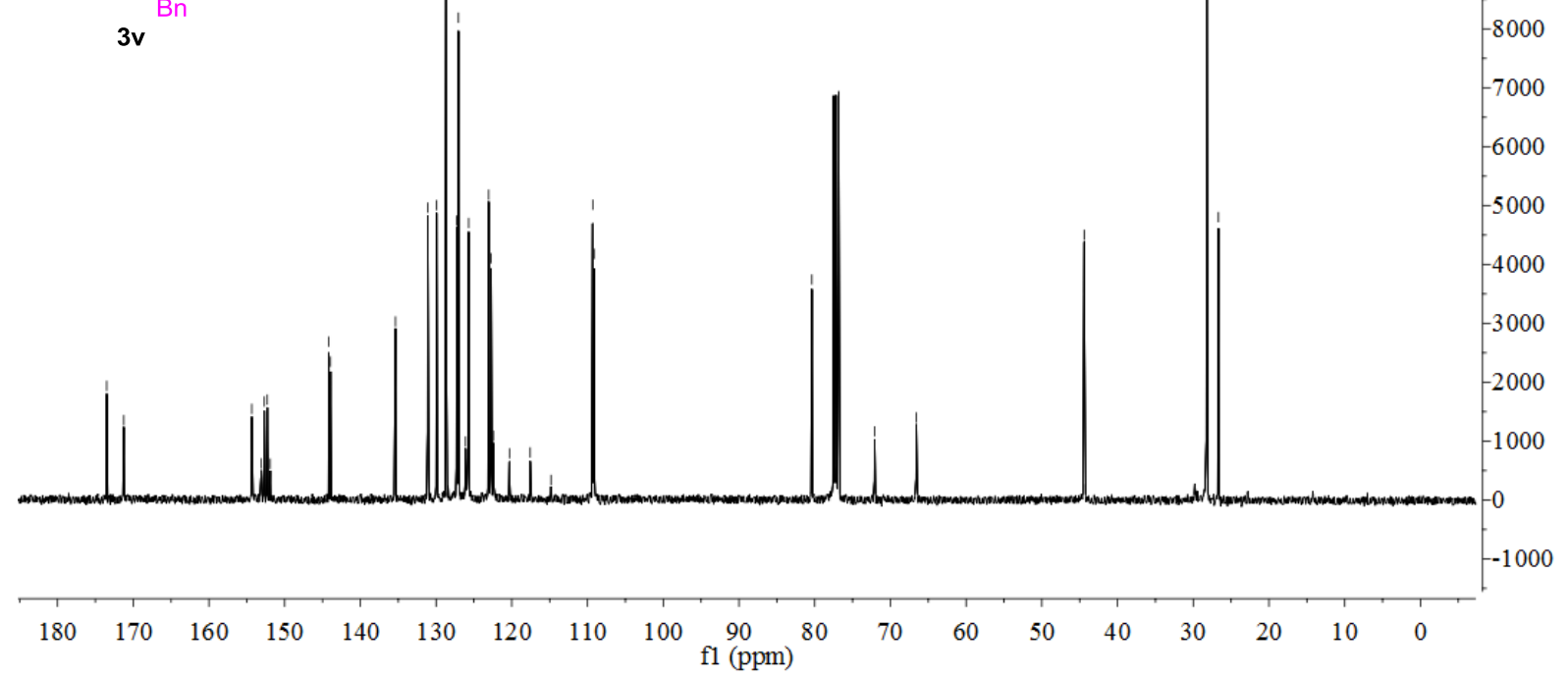

${ }^{19} \mathrm{~F}$ NMR of compound $3 \mathrm{v}\left(376 \mathrm{MHz}\right.$ in $\left.\mathrm{CDCl}_{3}\right)$

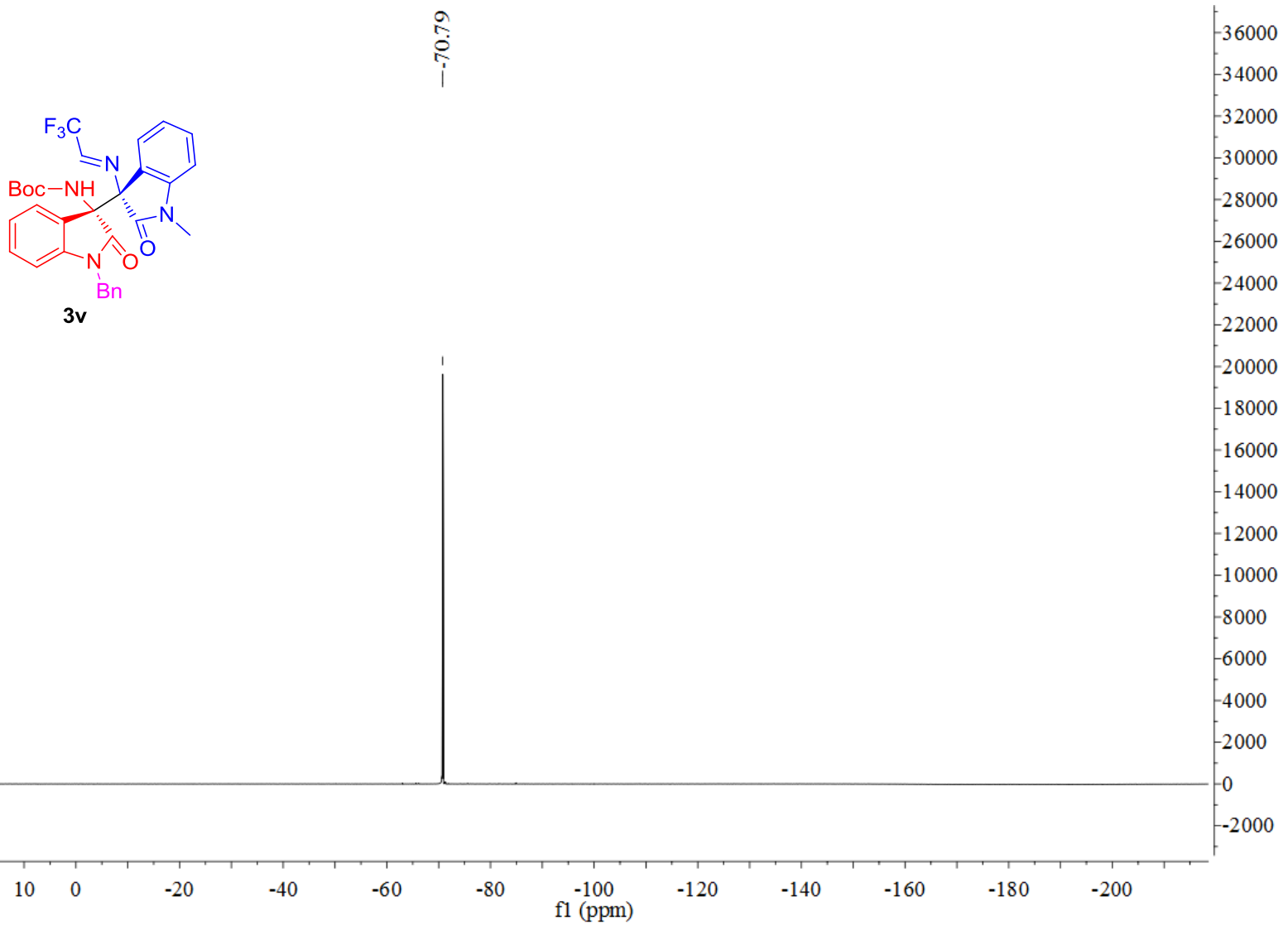


${ }^{1} \mathrm{H}$ NMR of compound $8 \mathrm{a}\left(400 \mathrm{MHz}\right.$ in $\left.\mathrm{CDCl}_{3}\right)$

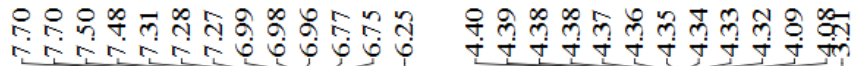<smiles>CCOC(=O)C(N=CC(F)(F)F)(NC(=O)C(F)(F)F)[C@]1(NC(=O)C(F)(F)F)C(=O)N(C)c2ccccc21</smiles>

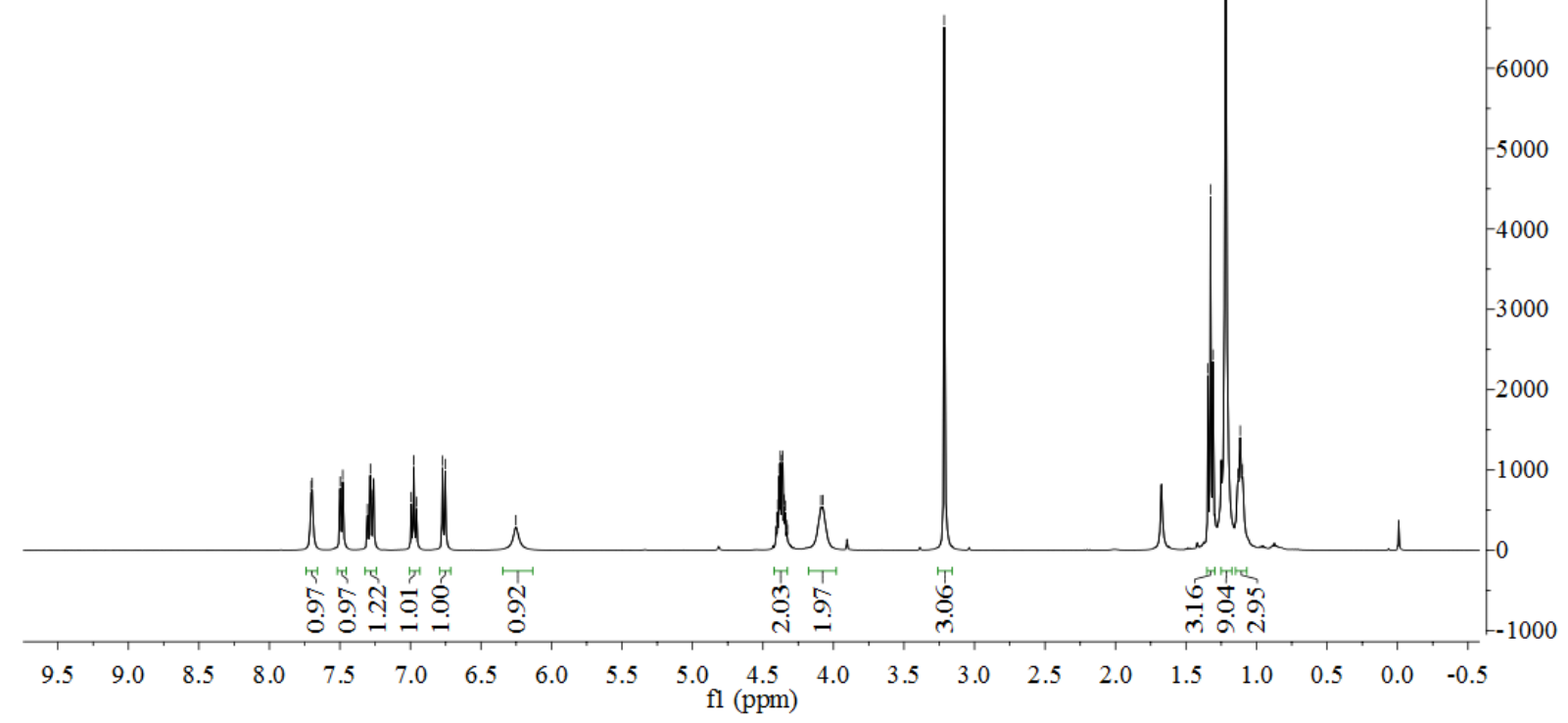

${ }^{13} \mathrm{C}$ NMR of compound $8 \mathrm{a}\left(101 \mathrm{MHz}\right.$ in $\left.\mathrm{CDCl}_{3}\right)$
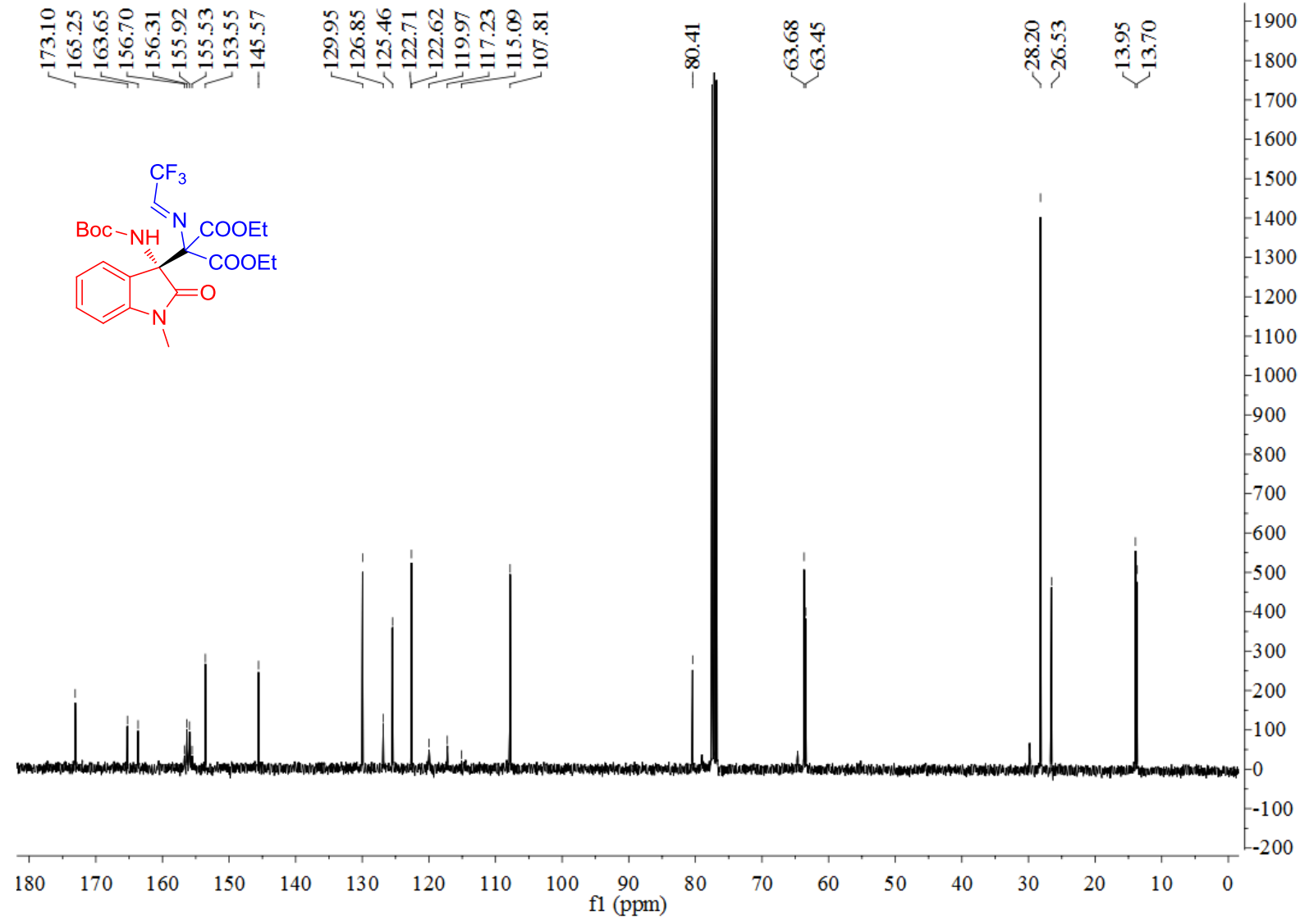


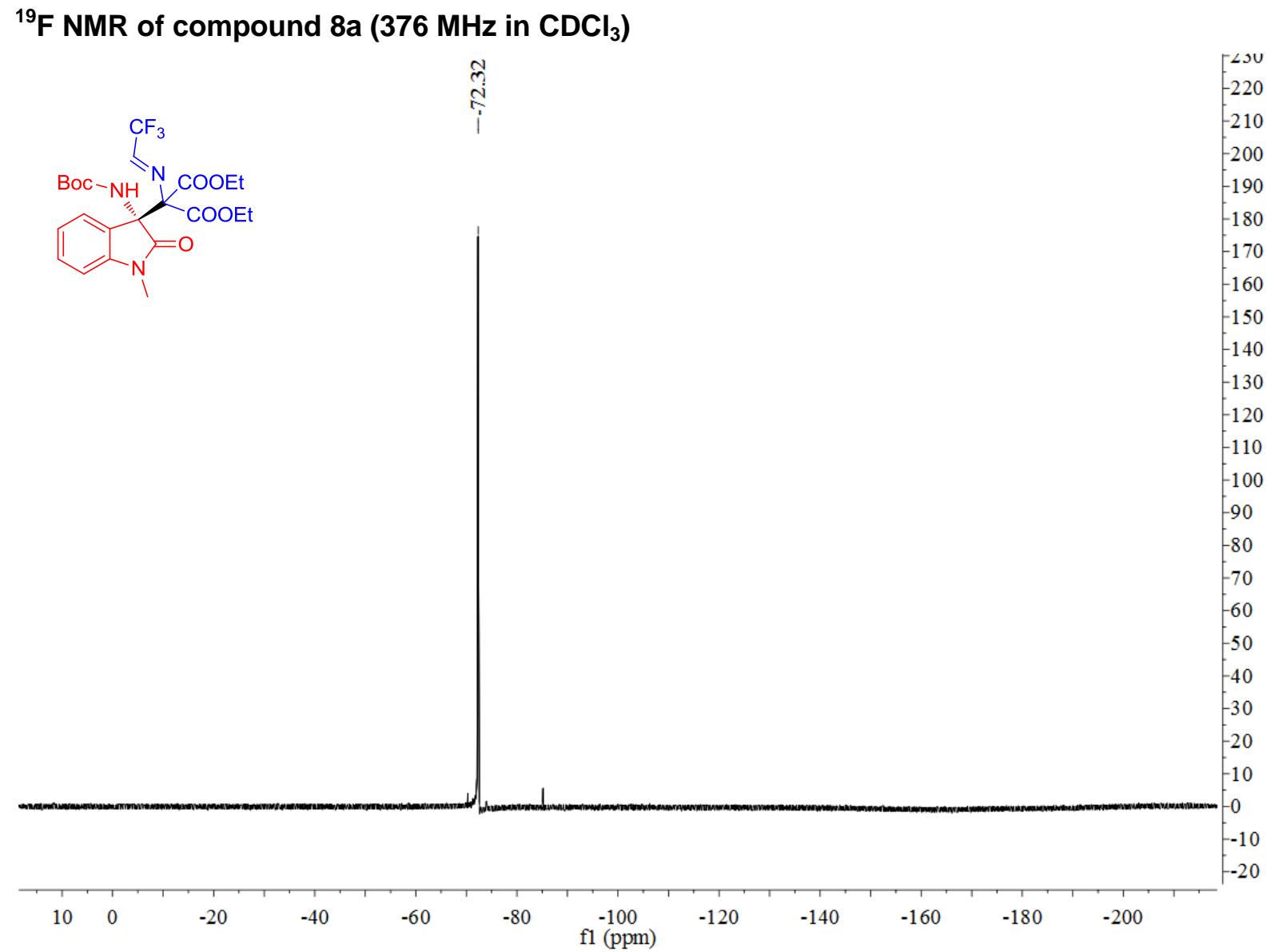

${ }^{1} \mathrm{H}$ NMR of compound $8 \mathrm{~b}\left(400 \mathrm{MHz}\right.$ in $\mathrm{CDCl}_{3}$ )

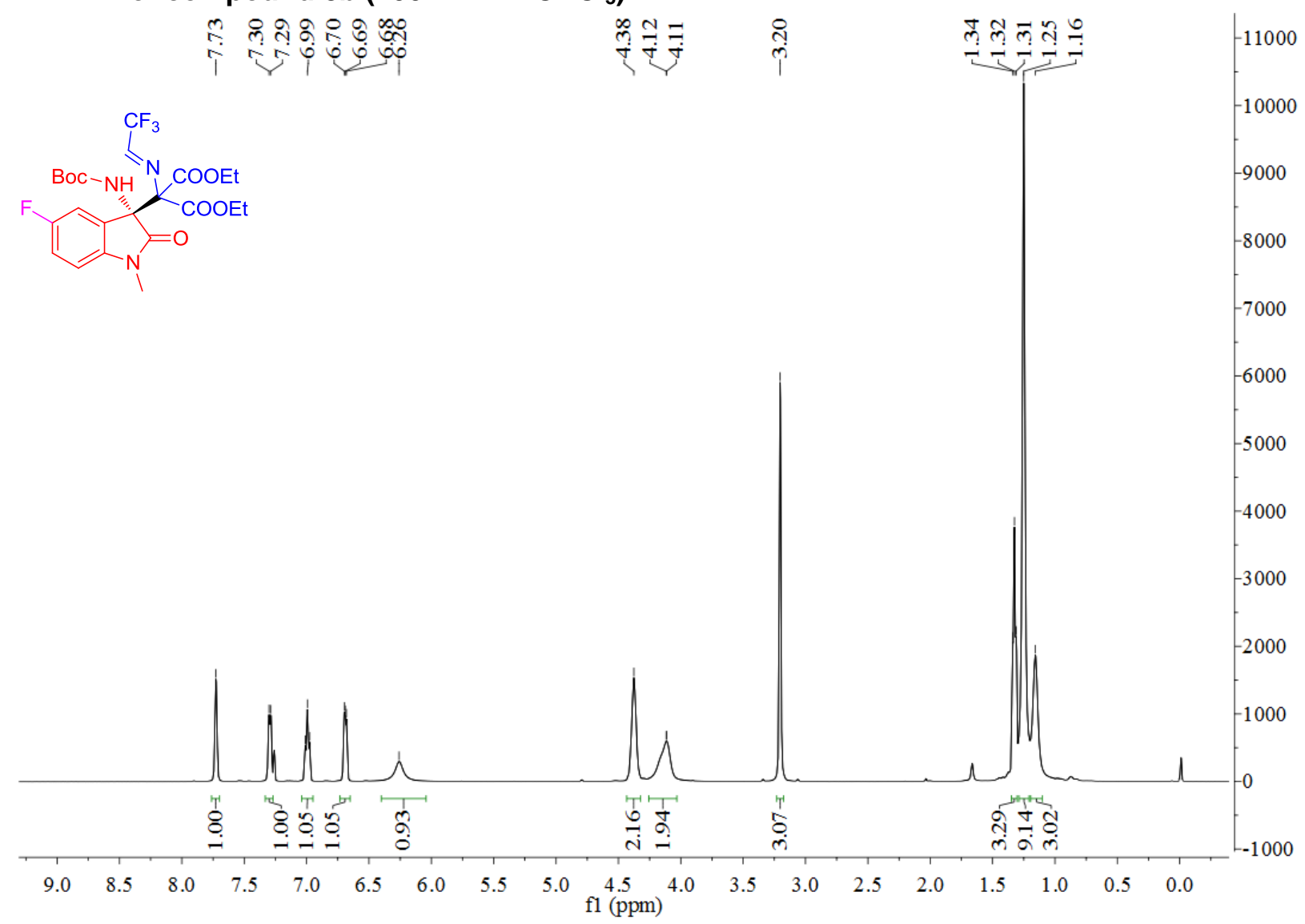




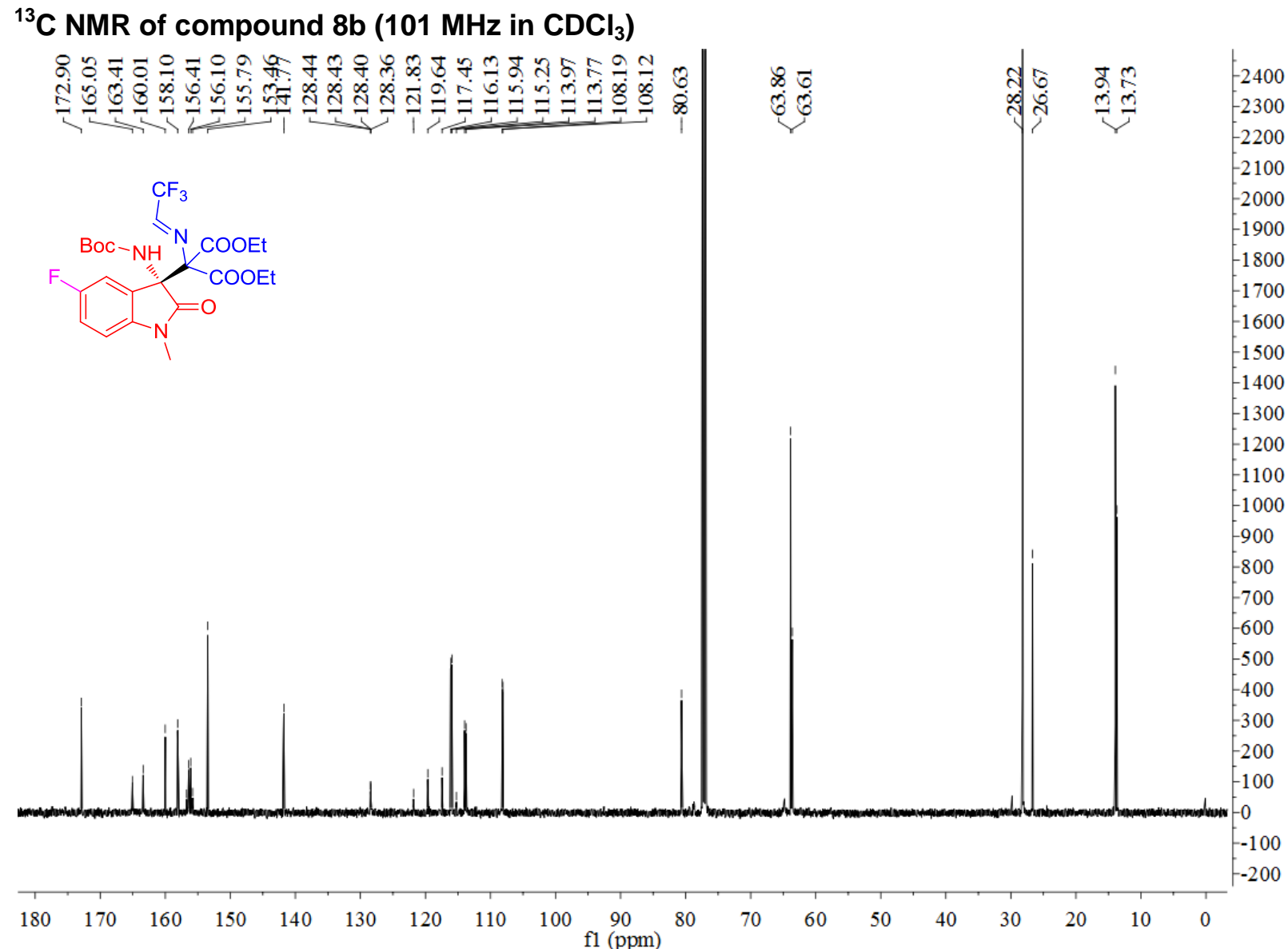

${ }^{19} \mathrm{~F} \mathrm{NMR}$ of compound $8 \mathrm{~b}\left(376 \mathrm{MHz}\right.$ in $\mathrm{CDCl}_{3}$ )

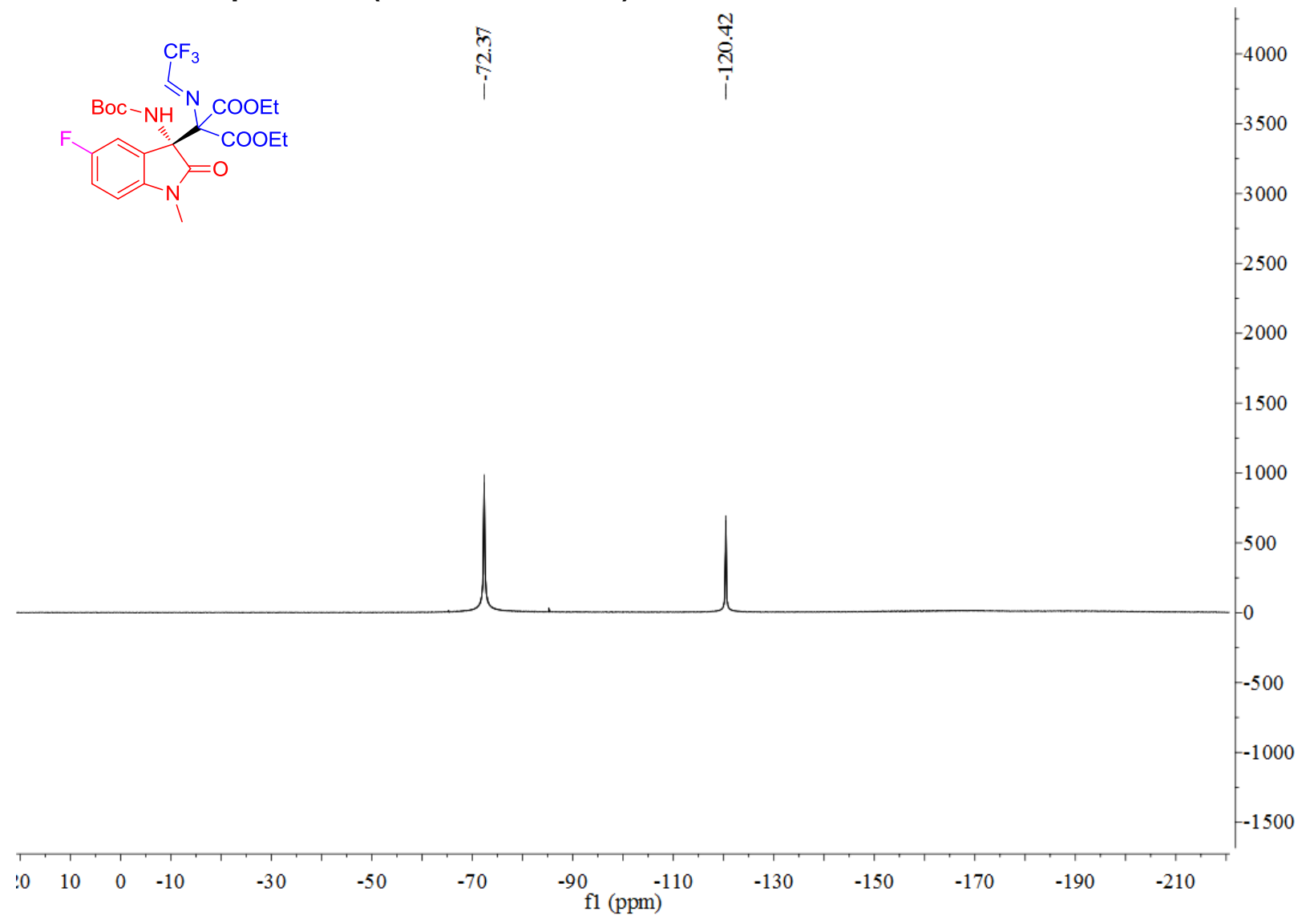


${ }^{1} \mathrm{H}$ NMR of compound $8 \mathrm{c}\left(400 \mathrm{MHz}\right.$ in $\left.\mathrm{CDCl}_{3}\right)$

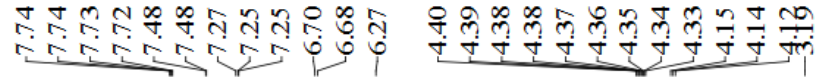<smiles>CCOC(=O)NC(NC=NC(F)(F)F)(C(=O)OCC)C1(NC(=O)OCc2ccccc2)C(=O)N(C)c2ccc(Cl)cc21</smiles>

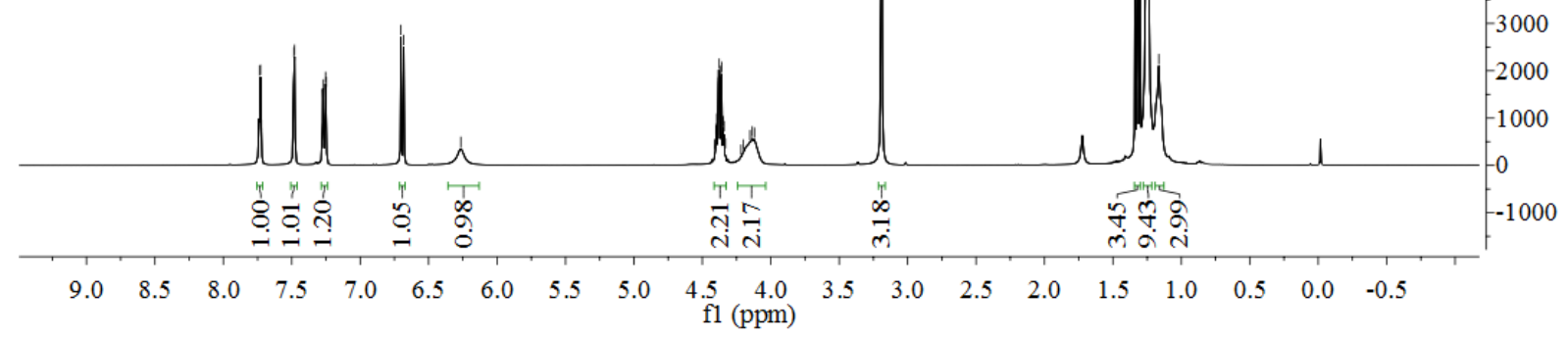

${ }^{13} \mathrm{C}$ NMR of compound $8 \mathrm{c}\left(101 \mathrm{MHz}\right.$ in $\left.\mathrm{CDCl}_{3}\right)$

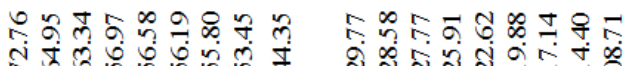

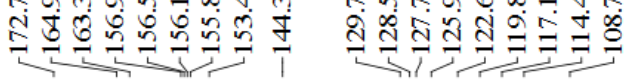

$\begin{array}{ll}1 & 85 \\ 8 & 80\end{array}$<smiles>CCOC(=O)N[C@@]1(C(C)(N=CC(F)(F)F)C(=O)OCC)C(=O)N(C)c2ccc(Cl)cc21</smiles>

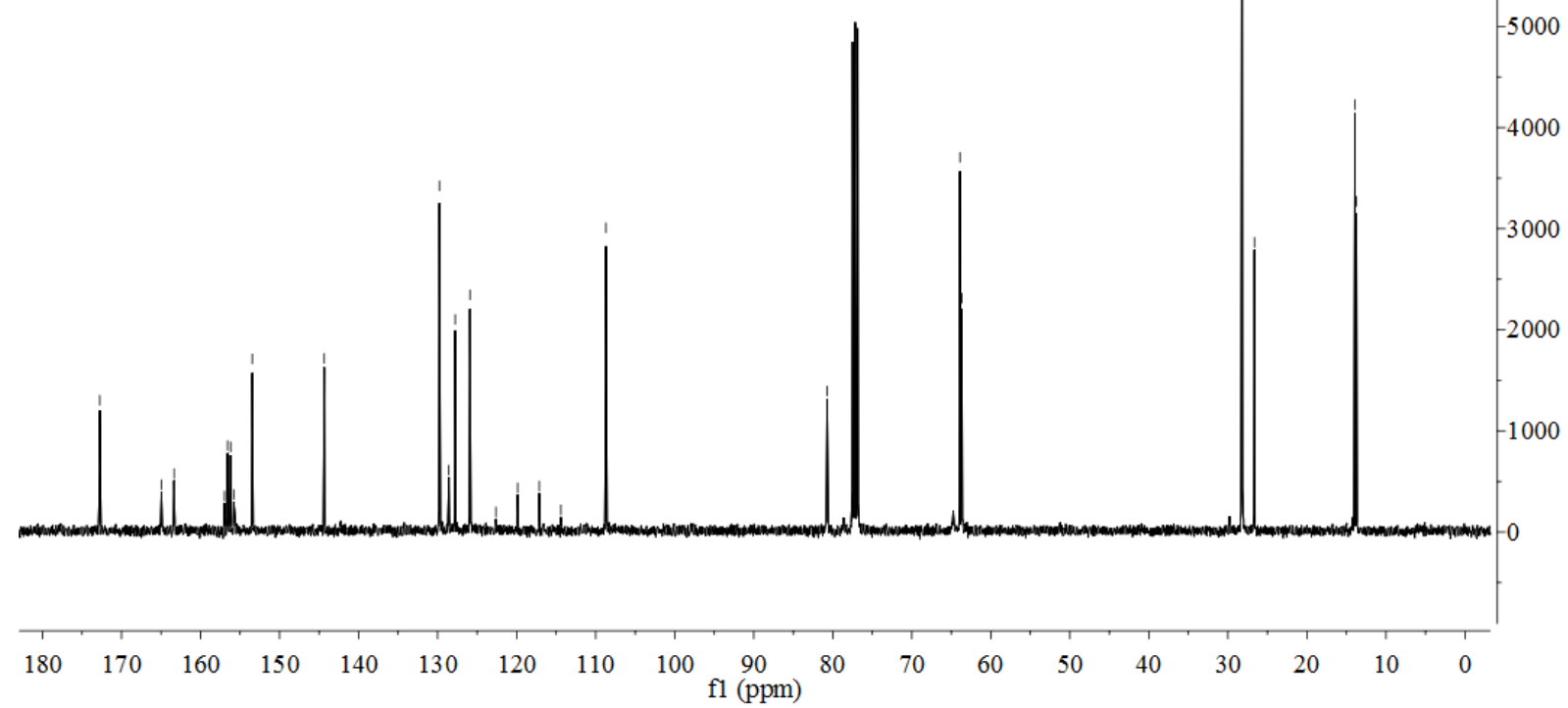


${ }^{19} \mathrm{~F}$ NMR of compound $8 \mathrm{c}\left(376 \mathrm{MHz}\right.$ in $\left.\mathrm{CDCl}_{3}\right)$

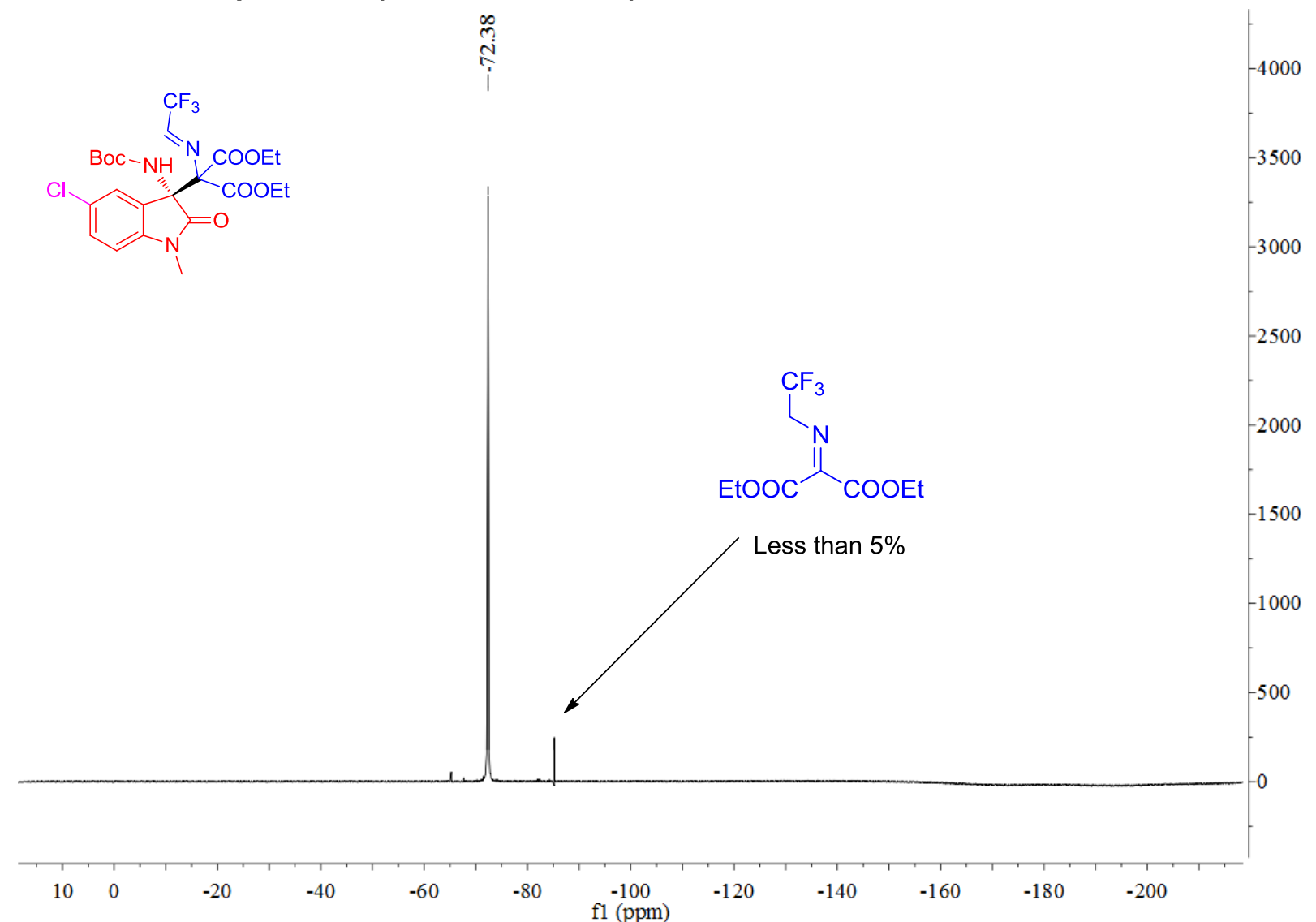

${ }^{1} \mathrm{H}$ NMR of compound $8 \mathrm{~d}\left(400 \mathrm{MHz}\right.$ in $\left.\mathrm{CDCl}_{3}\right)$

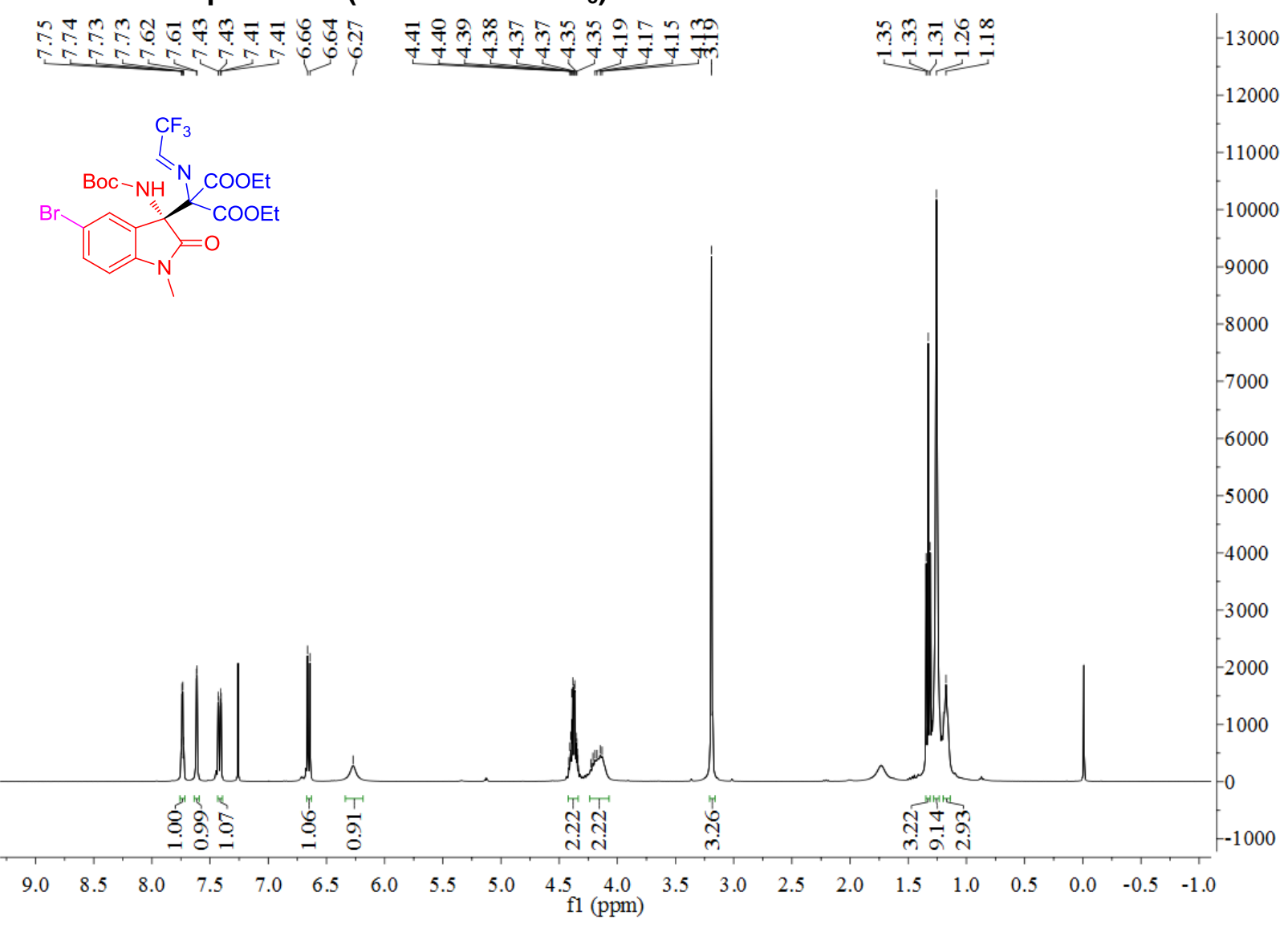


${ }^{13} \mathrm{C}$ NMR of compound $8 \mathrm{~d}\left(101 \mathrm{MHz}\right.$ in $\left.\mathrm{CDCl}_{3}\right)$

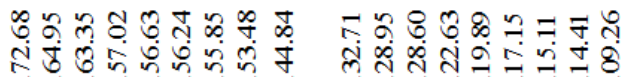

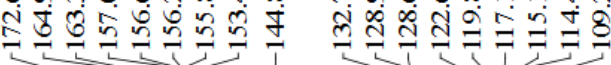

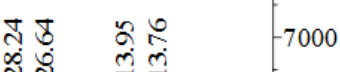<smiles>CCOC(=O)C(N=CC(F)(F)F)(C(=O)OCC)C1(NC(=O)c2ccccc2)C(=O)N(C)c2ccc(Br)cc21</smiles>

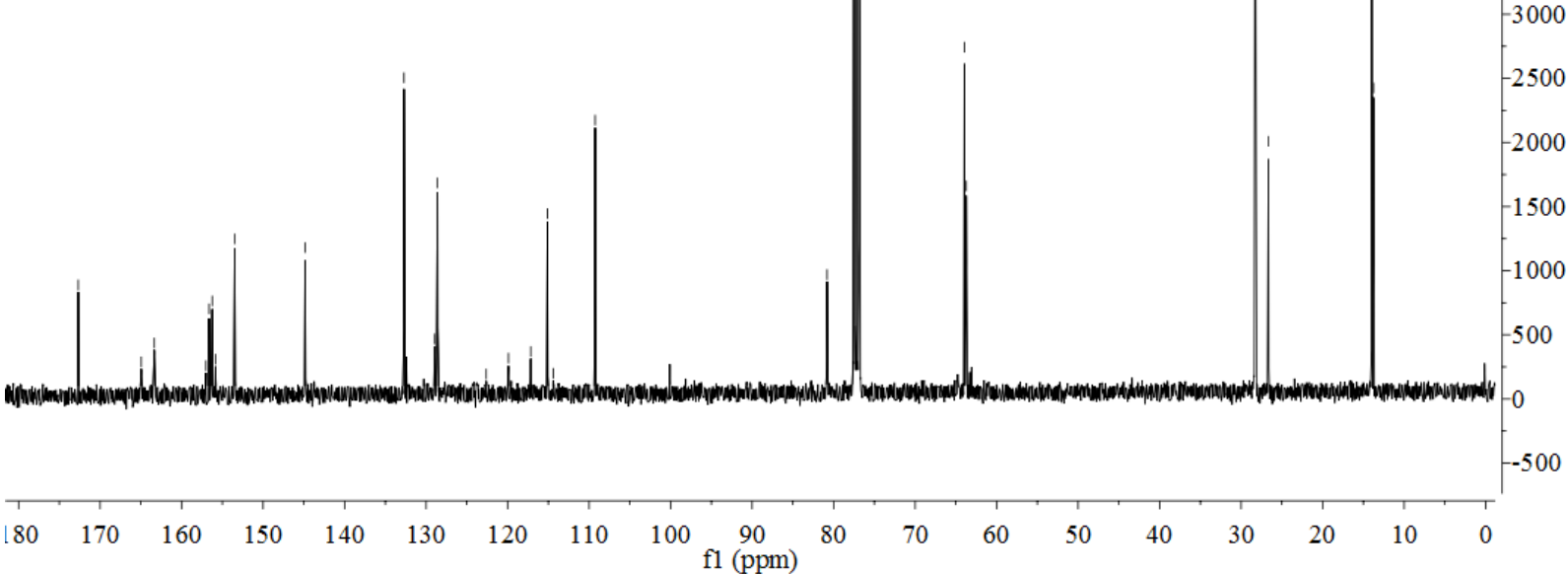

${ }^{19} \mathrm{~F}$ NMR of compound $8 \mathrm{~d}\left(376 \mathrm{MHz}\right.$ in $\left.\mathrm{CDCl}_{3}\right)$<smiles>CCOC(=O)C(C)(C(=O)NCC(F)(F)F)C1(N=CC(=O)c2ccccc2)C(=O)N(C)c2ccc(Br)cc21</smiles><smiles>[C+]1=CC=CC=1</smiles>

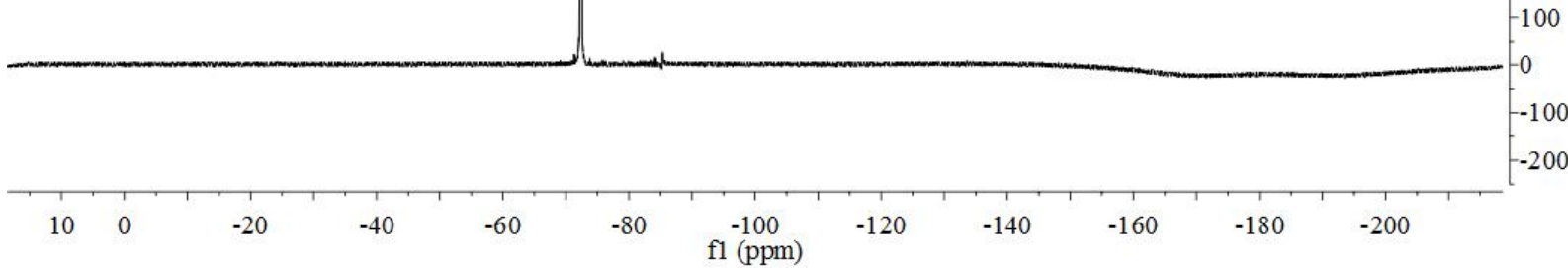


${ }^{1} \mathrm{H}$ NMR of compound $8 \mathrm{e}\left(400 \mathrm{MHz}\right.$ in $\left.\mathrm{CDCl}_{3}\right)$

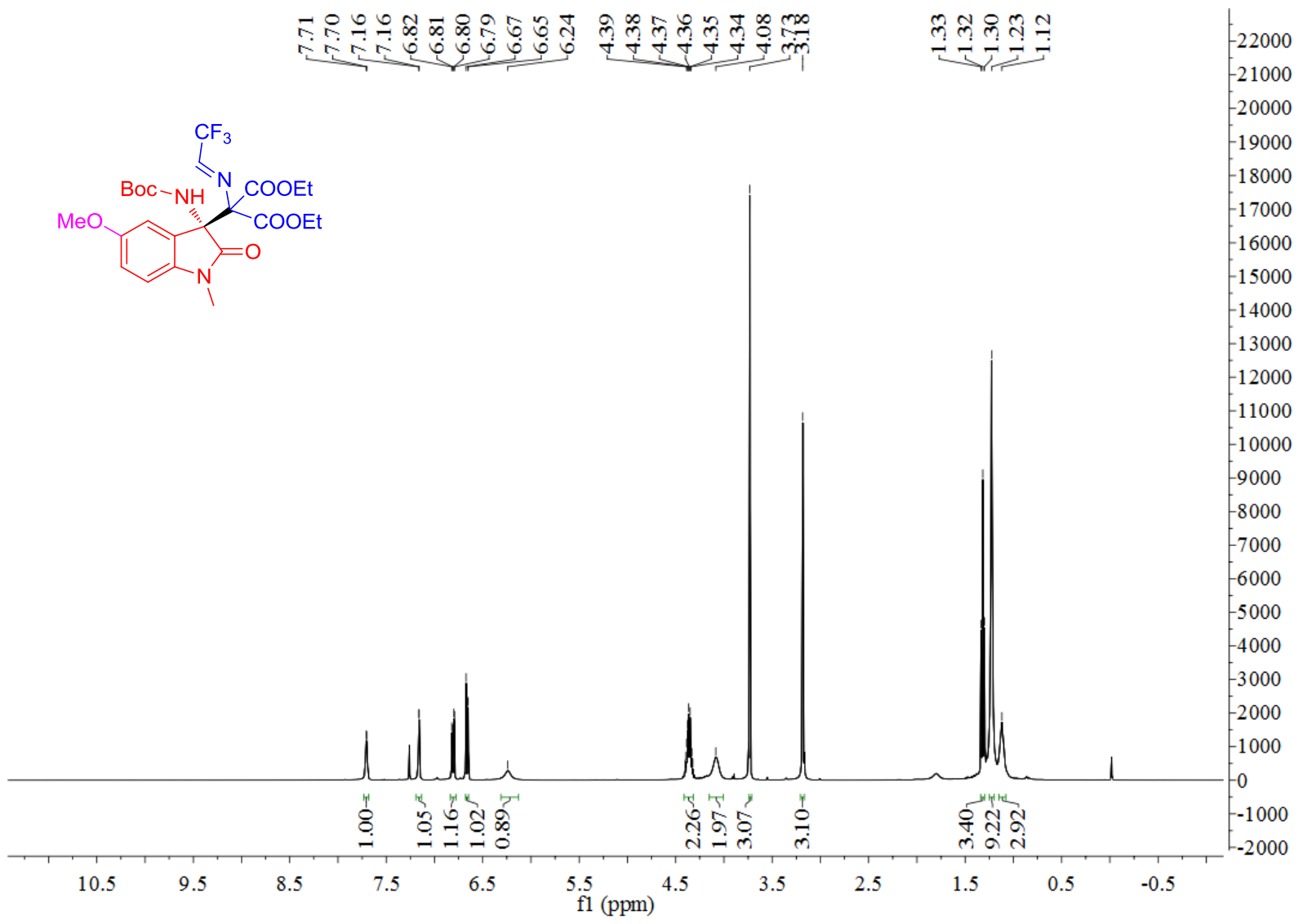

${ }^{13} \mathrm{C}$ NMR of compound $8 \mathrm{e}\left(101 \mathrm{MHz}\right.$ in $\left.\mathrm{CDCl}_{3}\right)$

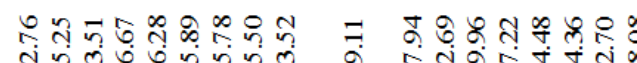

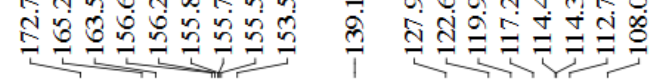

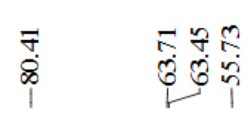

(N)

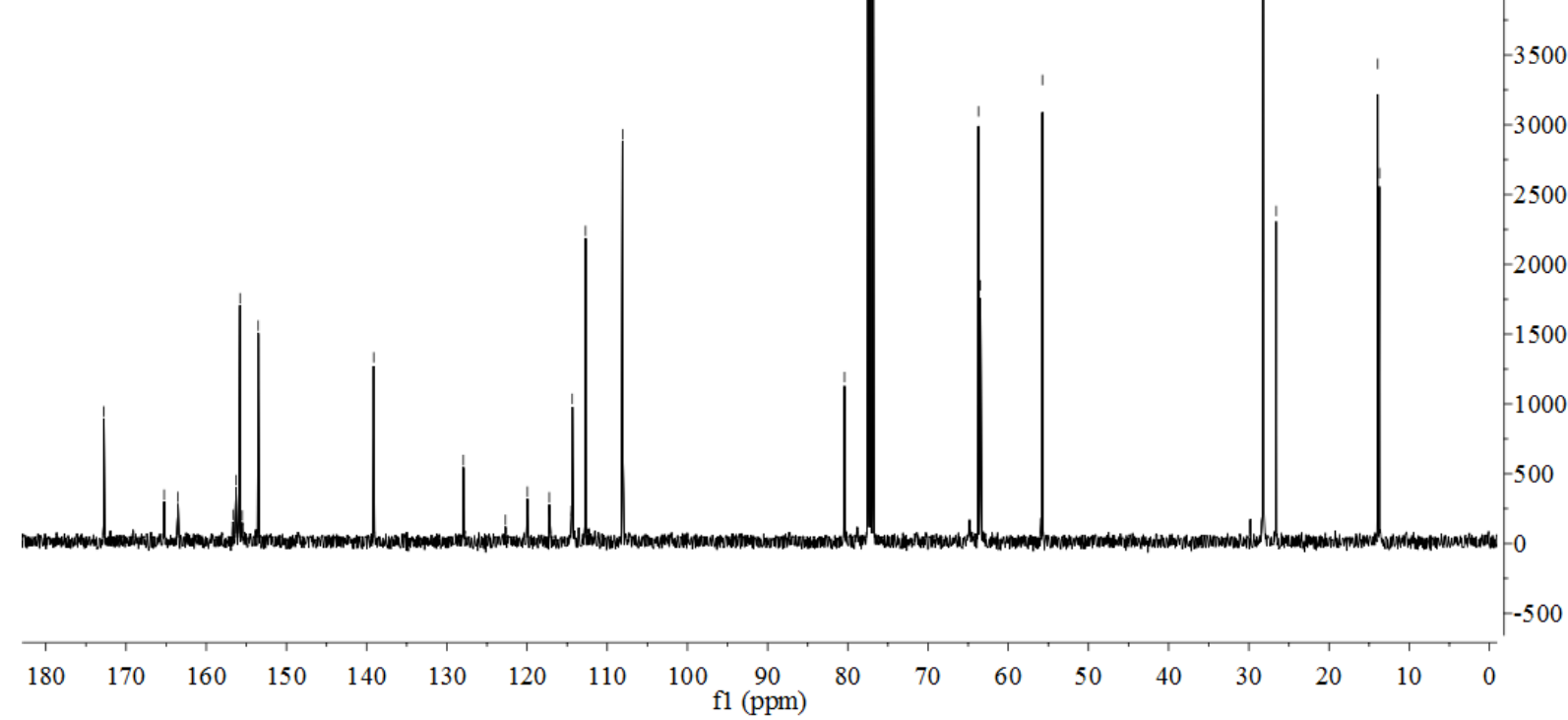


${ }^{19} \mathrm{~F}$ NMR of compound $8 \mathrm{e}\left(376 \mathrm{MHz}\right.$ in $\left.\mathrm{CDCl}_{3}\right)$

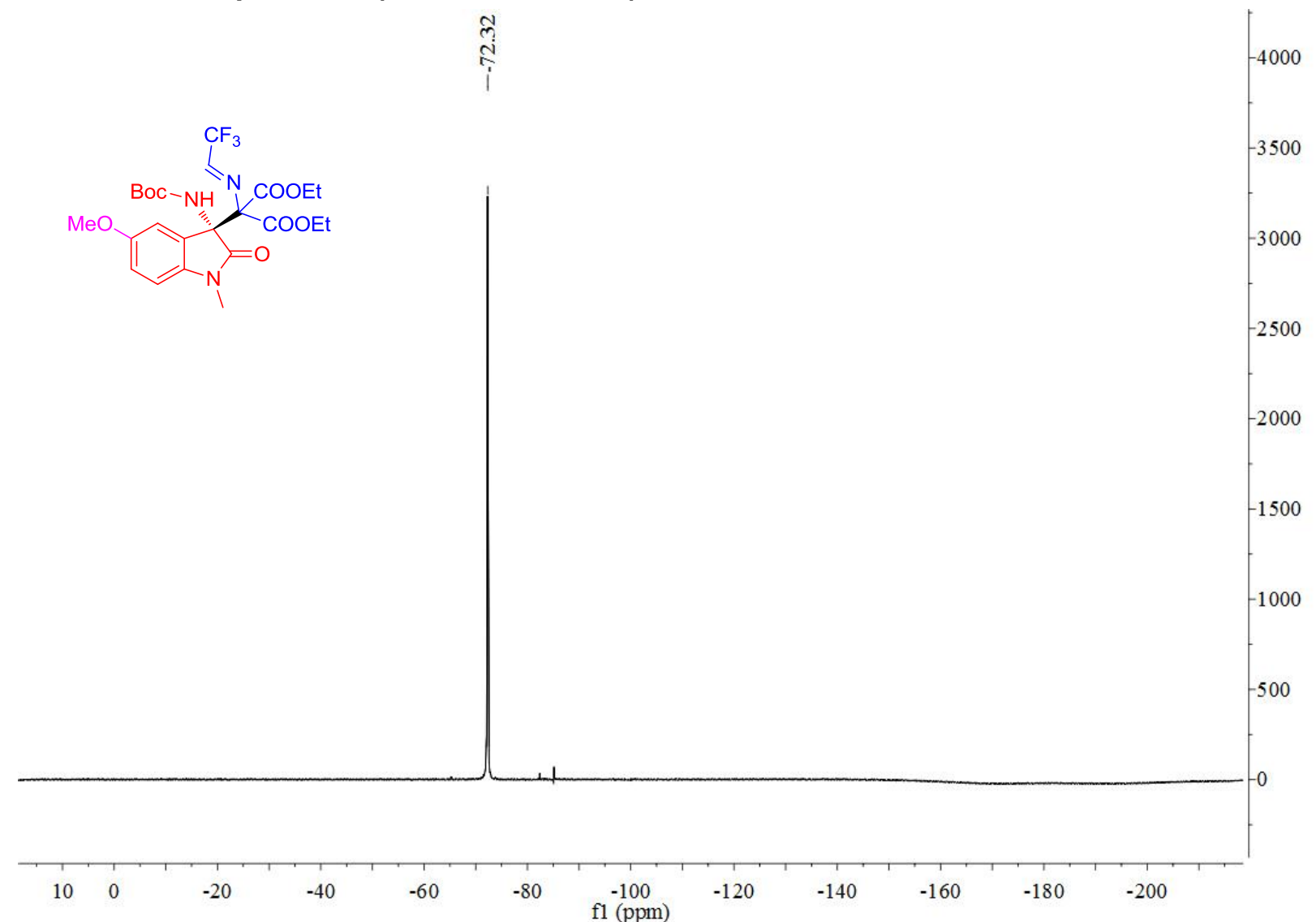

${ }^{1} \mathrm{H}$ NMR of compound $8 f\left(400 \mathrm{MHz}\right.$ in $\left.\mathrm{CDCl}_{3}\right)$

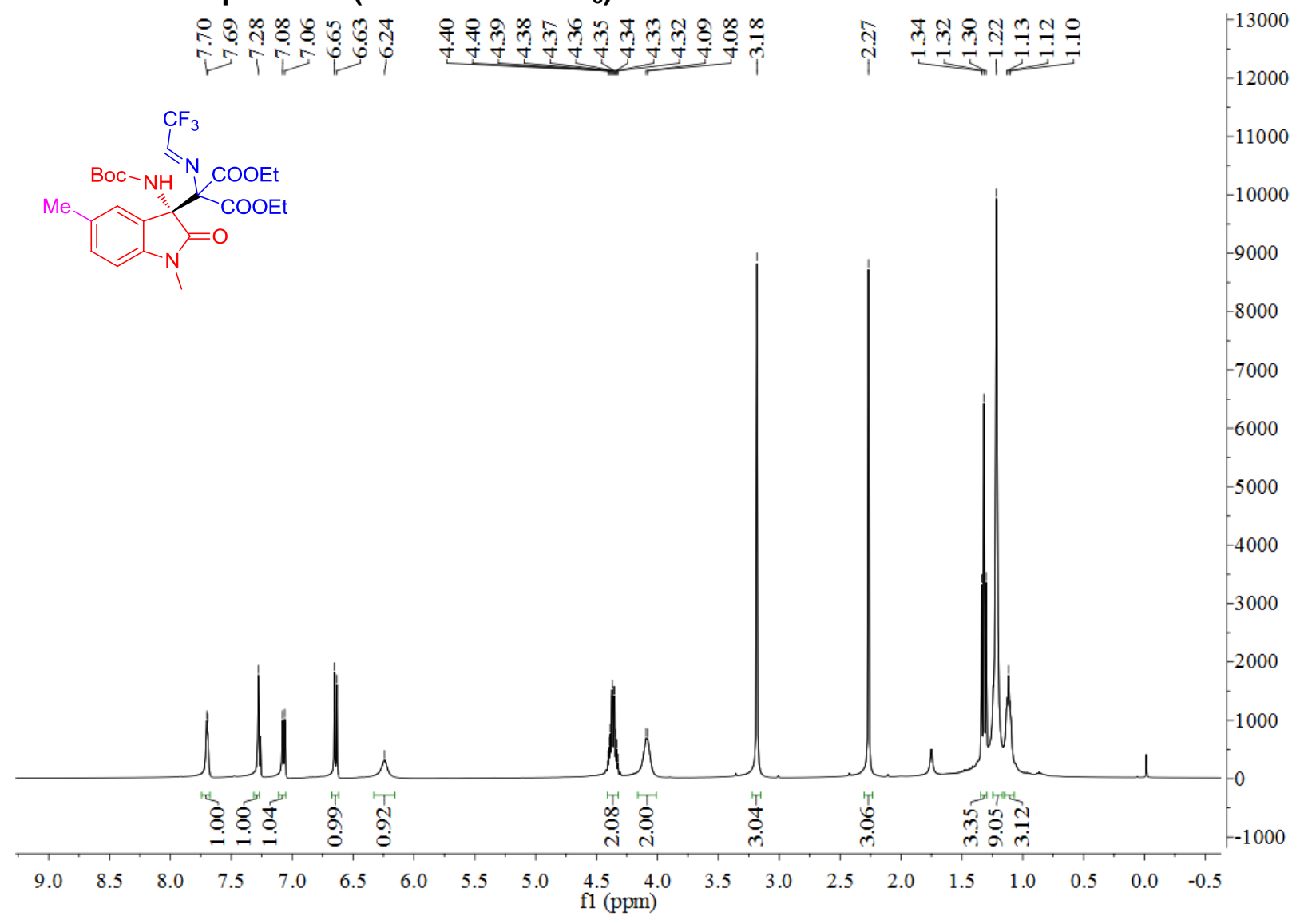


${ }^{13} \mathrm{C}$ NMR of compound $8 f\left(101 \mathrm{MHz}\right.$ in $\mathrm{CDCl}_{3}$ )

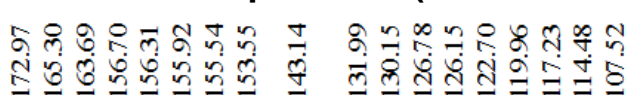

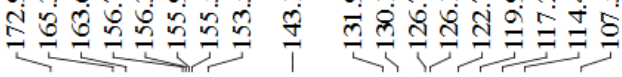

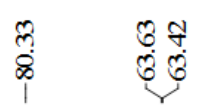

के लि ही

$-9000$

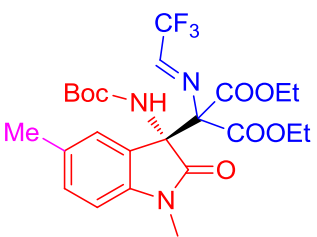

000

6000

$-5000$

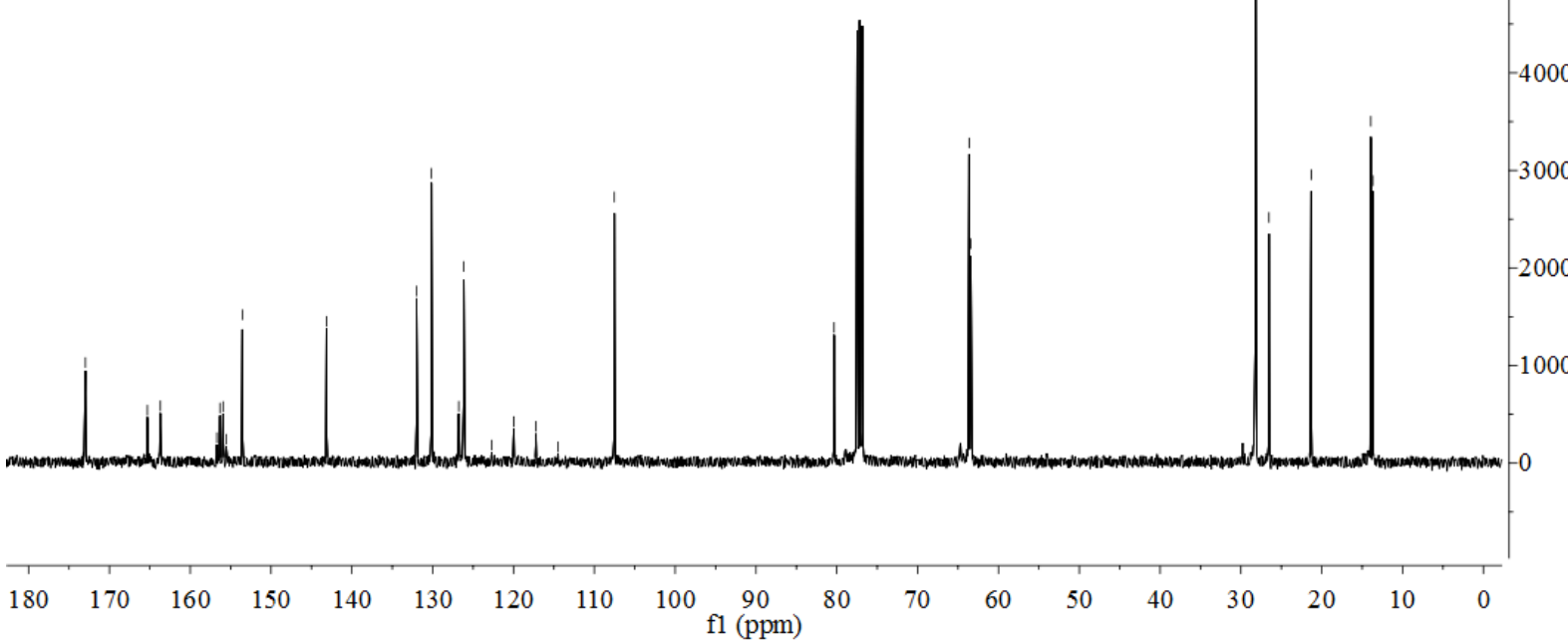

${ }^{19} \mathrm{~F}$ NMR of compound $8 f\left(376 \mathrm{MHz}\right.$ in $\left.\mathrm{CDCl}_{3}\right)$

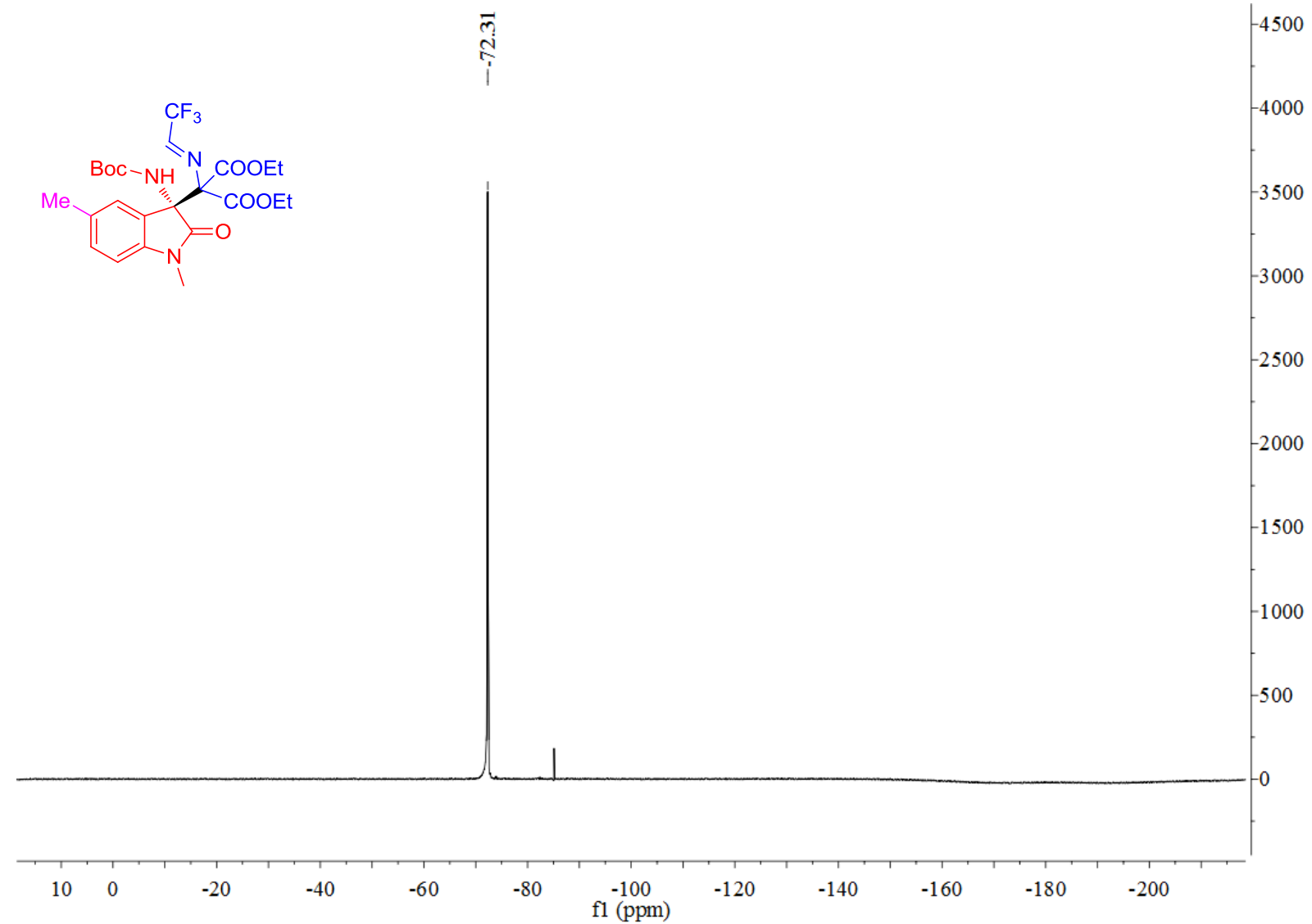


${ }^{1} \mathrm{H}$ NMR of compound $8 \mathrm{~g}\left(400 \mathrm{MHz}\right.$ in $\left.\mathrm{CDCl}_{3}\right)$

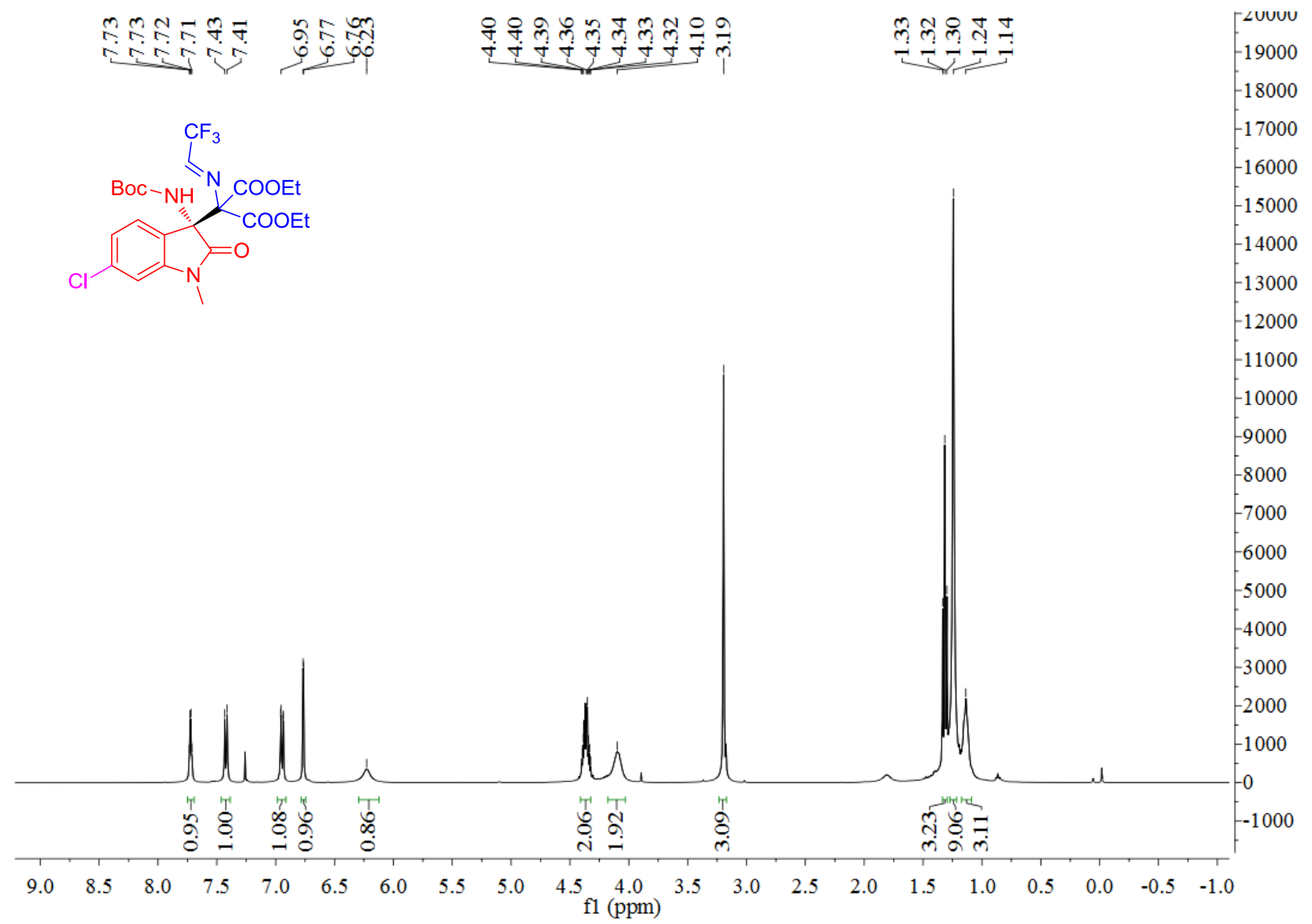

${ }^{13} \mathrm{C}$ NMR of compound $8 \mathrm{~g}\left(101 \mathrm{MHz}\right.$ in $\left.\mathrm{CDCl}_{3}\right)$

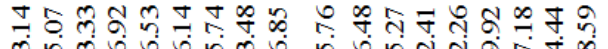

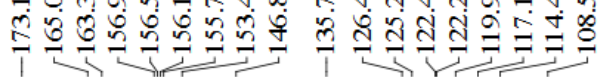

8
$\dot{\infty} 1$

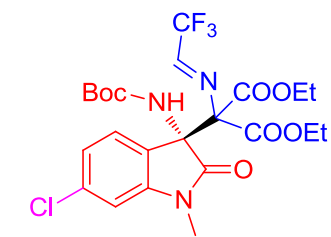

COOEt

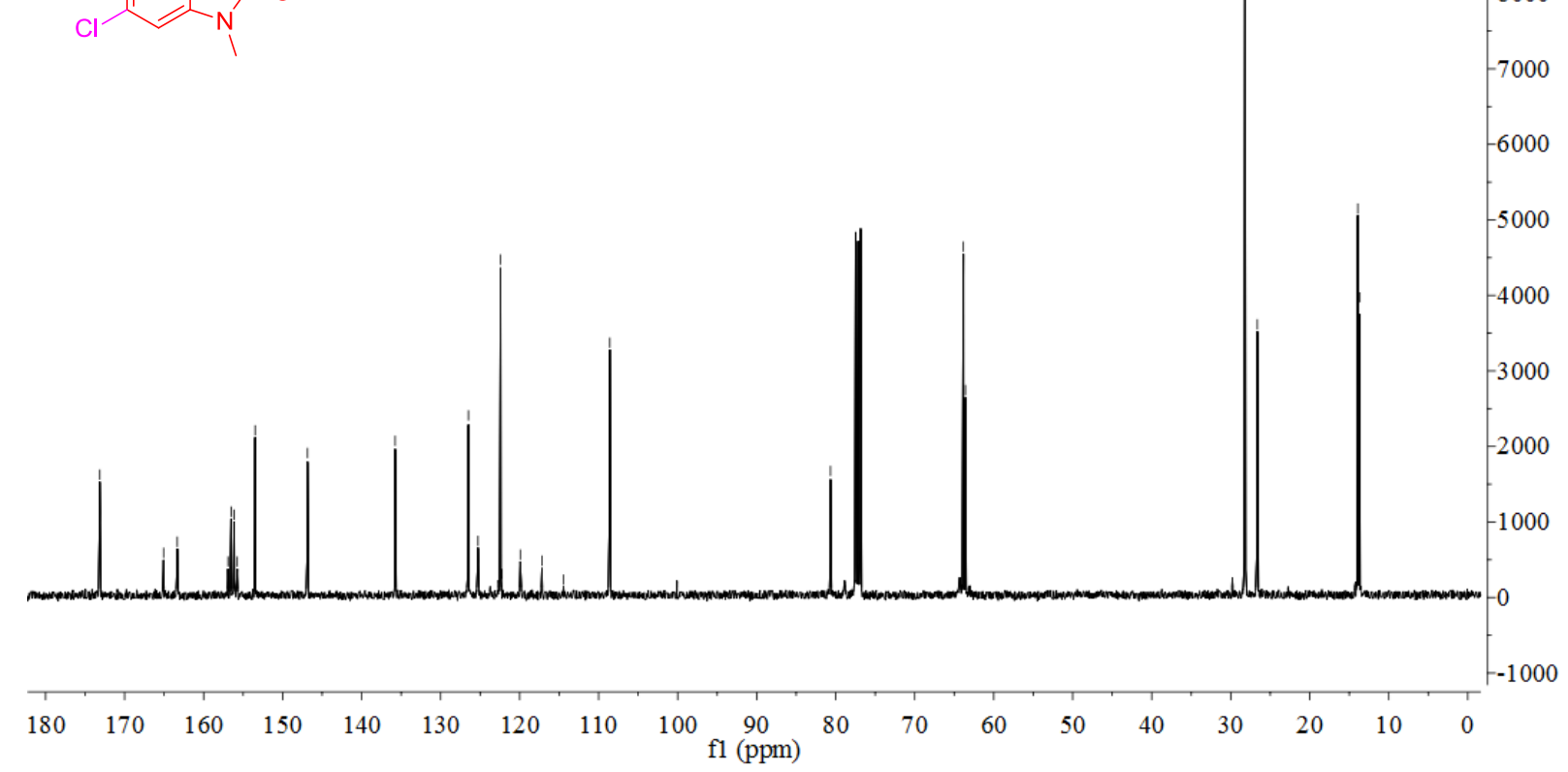


${ }^{19} \mathrm{~F}$ NMR of compound $8 \mathrm{~g}\left(376 \mathrm{MHz}\right.$ in $\left.\mathrm{CDCl}_{3}\right)$
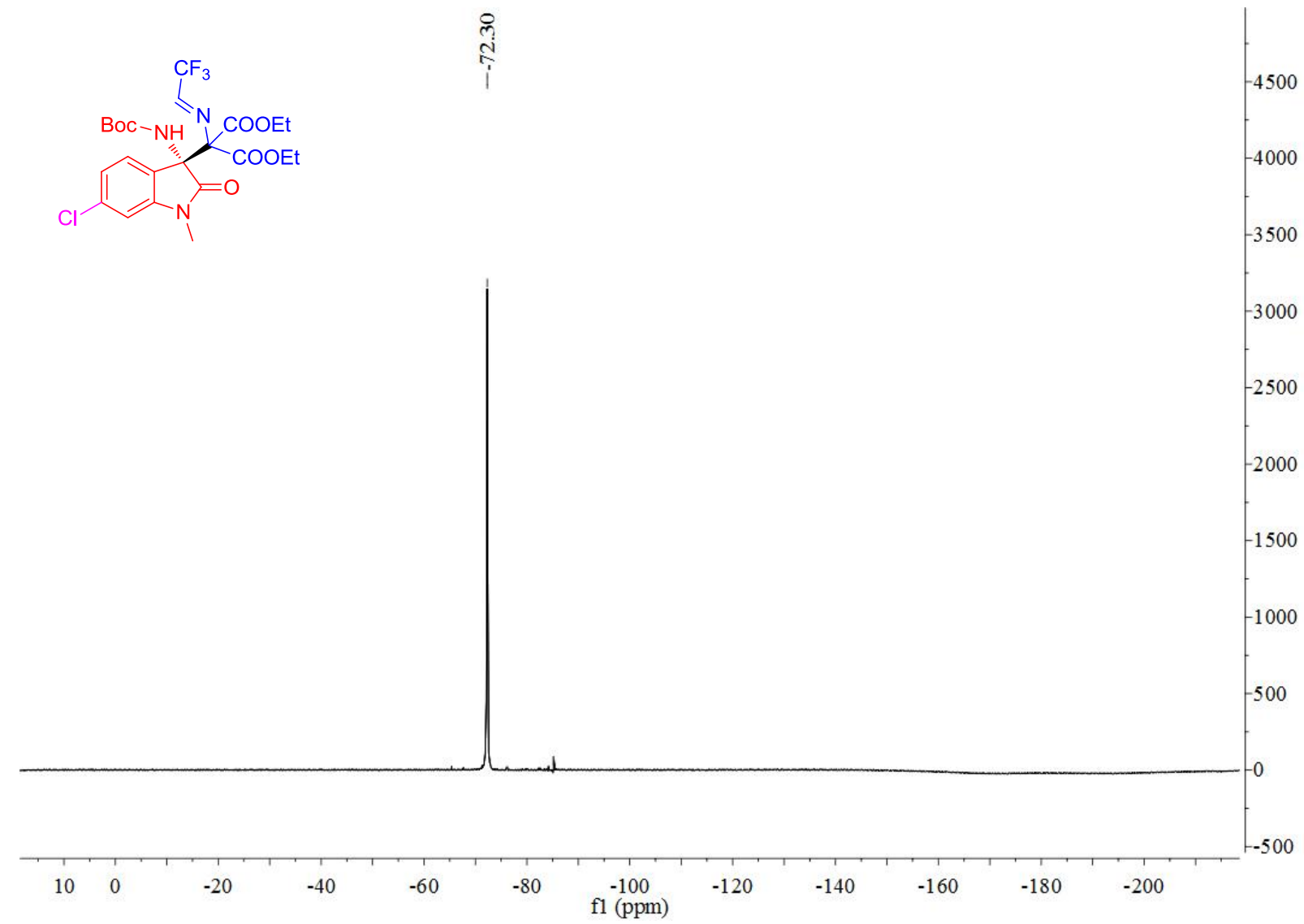

${ }^{1} \mathrm{H}$ NMR of compound $8 \mathrm{~h}\left(500 \mathrm{MHz}\right.$ in $\left.\mathrm{CDCl}_{3}\right)$

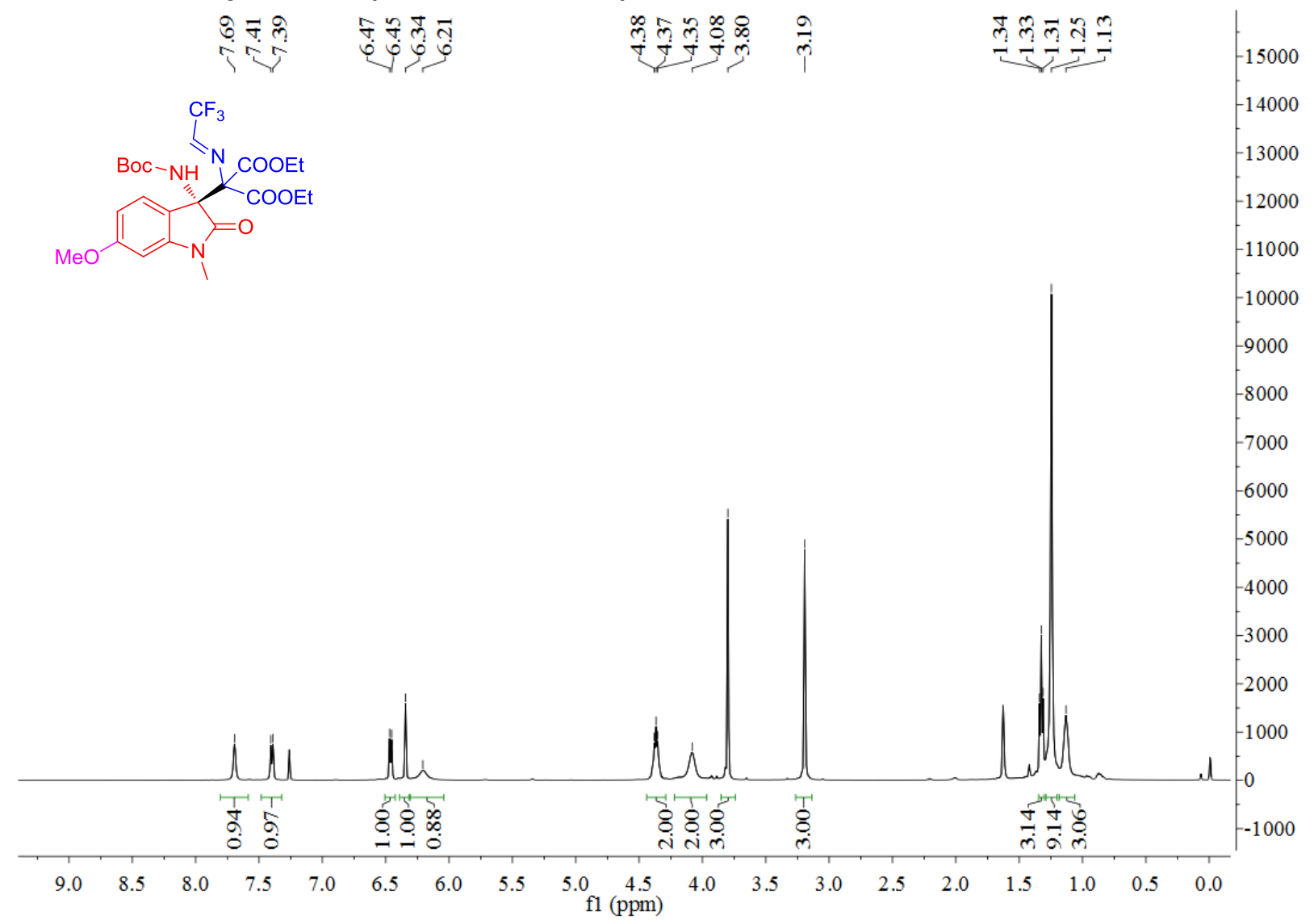


${ }^{13} \mathrm{C}$ NMR of compound $8 \mathrm{~h}\left(126 \mathrm{MHz}\right.$ in $\mathrm{CDCl}_{3}$ )

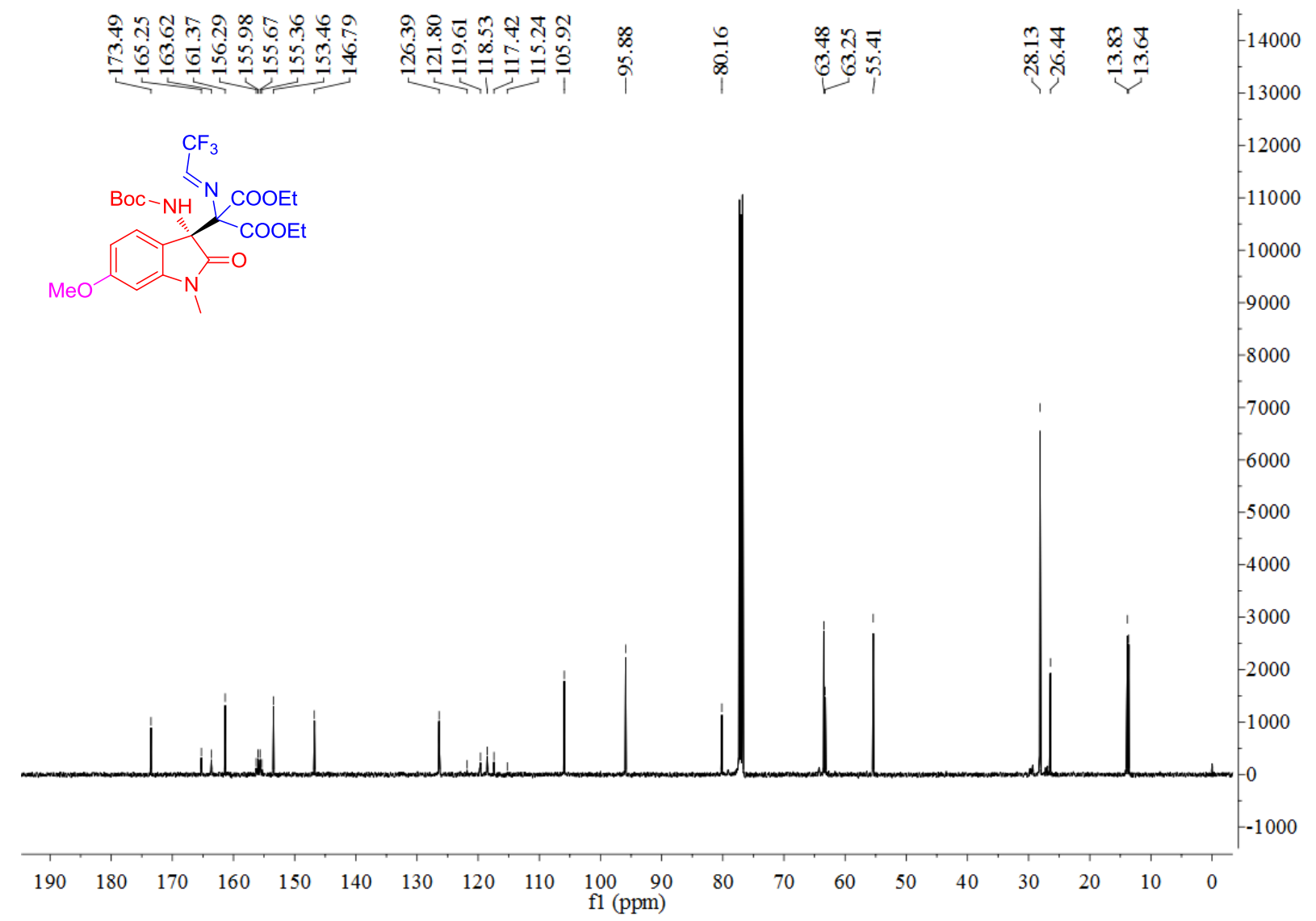

${ }^{19} \mathrm{~F} \mathrm{NMR}$ of compound $8 \mathrm{~h}\left(376 \mathrm{MHz}\right.$ in $\mathrm{CDCl}_{3}$ )

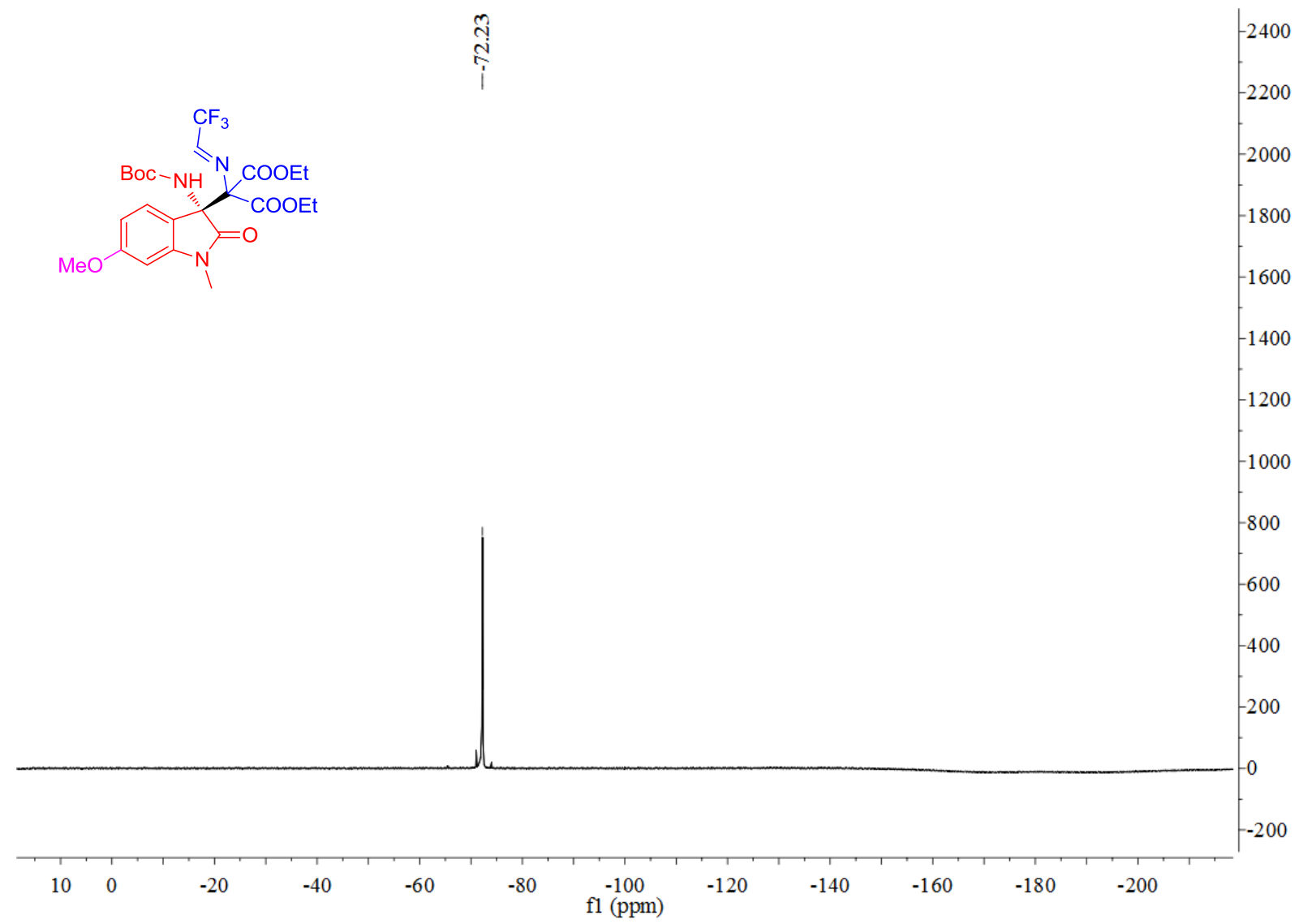


${ }^{1} \mathrm{H}$ NMR of compound $8 \mathrm{i}\left(400 \mathrm{MHz}\right.$ in $\left.\mathrm{CDCl}_{3}\right)$

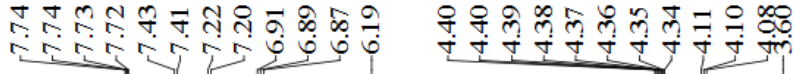<smiles>CCOC(=O)NC(N=CC(F)(F)F)(C(=O)OCC)C1(NC(=O)OCc2ccccc2)C(=O)N(C)c2c(Cl)cccc21</smiles>

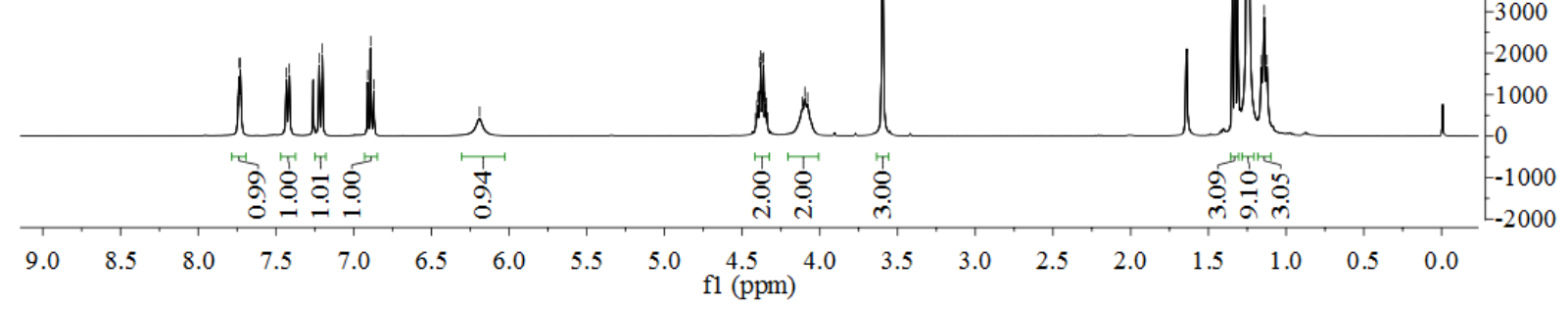

${ }^{13} \mathrm{C}$ NMR of compound $8 \mathrm{i}\left(101 \mathrm{MHz}\right.$ in $\mathrm{CDCl}_{3}$ )

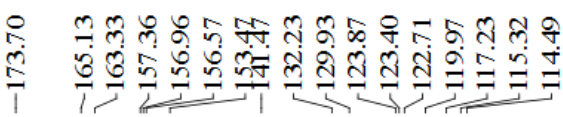<smiles>CCOC(=O)NC(N=CC(F)(F)F)(C(=O)OCC)[C@@]1(NC(=O)OCC)C(=O)N(C)c2c(Cl)cccc21</smiles>

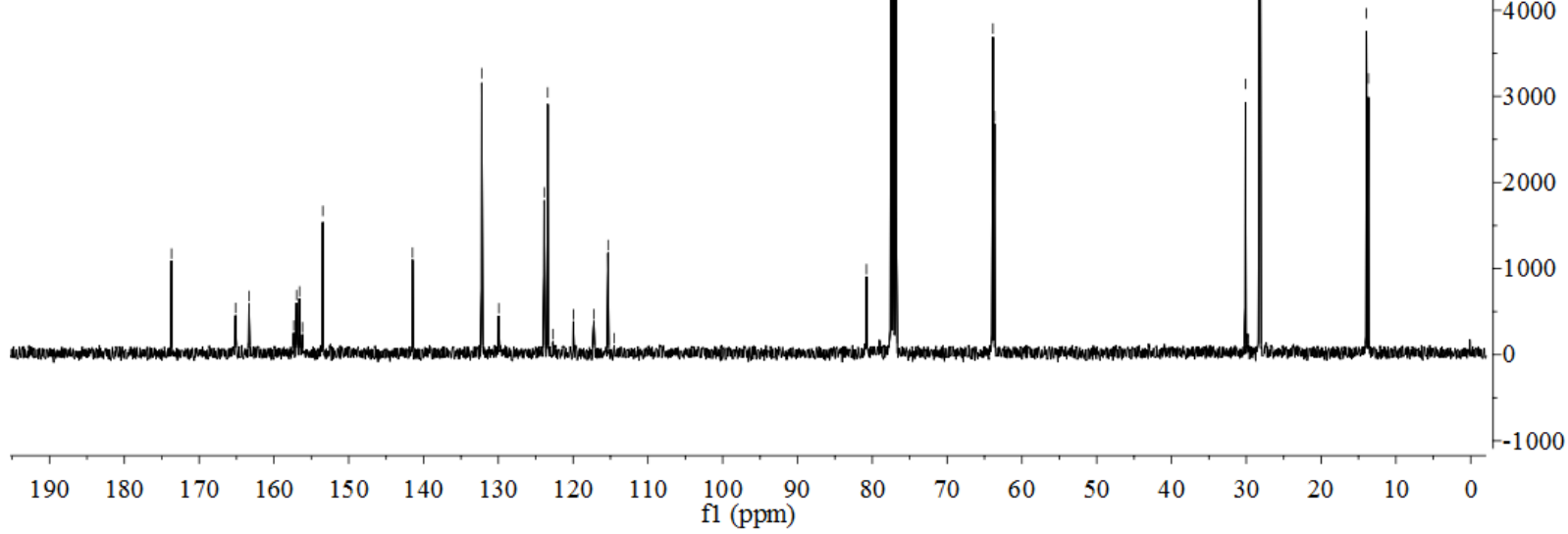


${ }^{19} \mathrm{~F}$ NMR of compound $8 \mathrm{i}\left(376 \mathrm{MHz}\right.$ in $\left.\mathrm{CDCl}_{3}\right)$

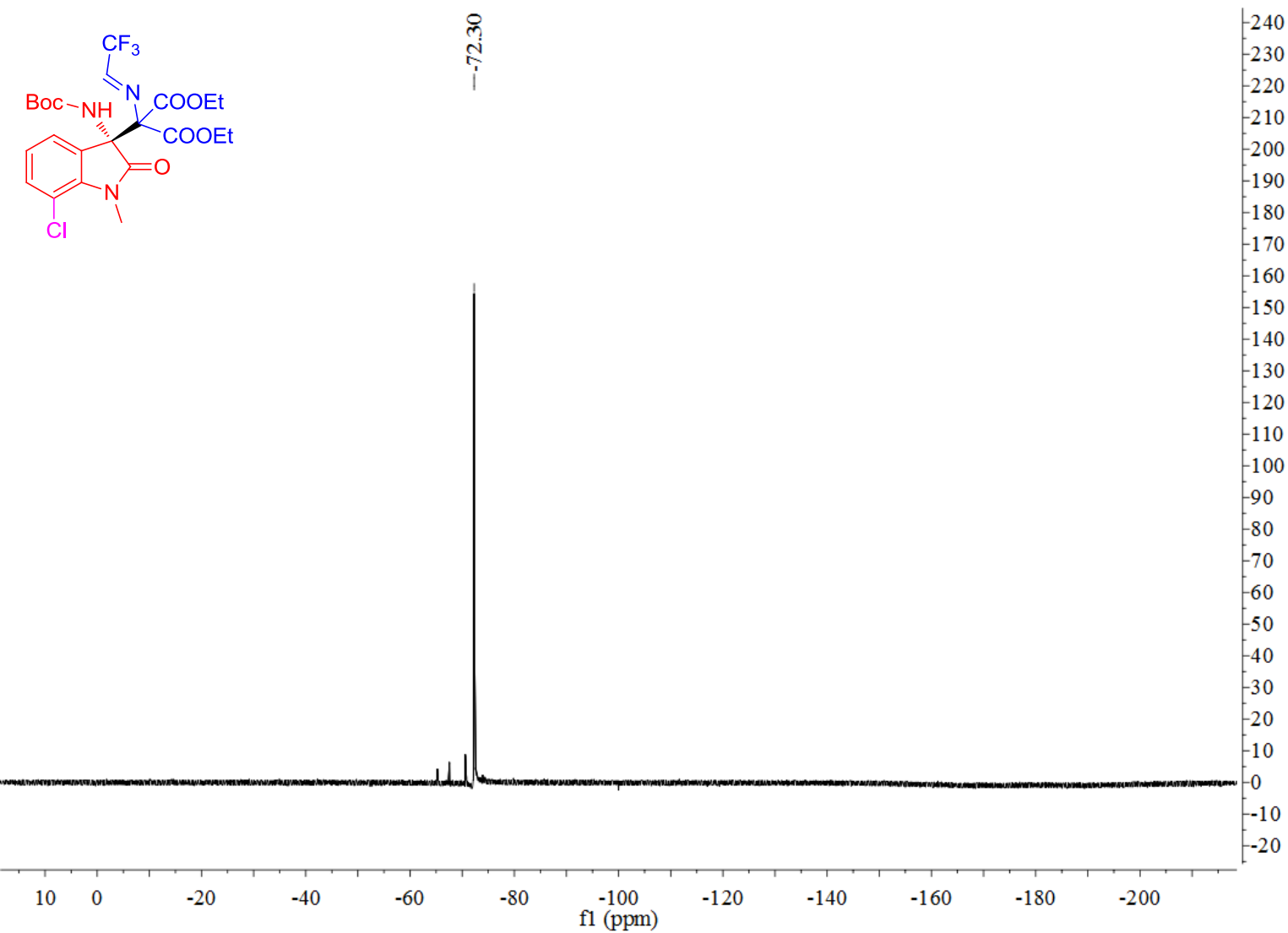

${ }^{1} \mathrm{H}$ NMR of compound $8 \mathrm{j}$ (400 $\mathrm{MHz}$ in $\mathrm{CDCl}_{3}$ )

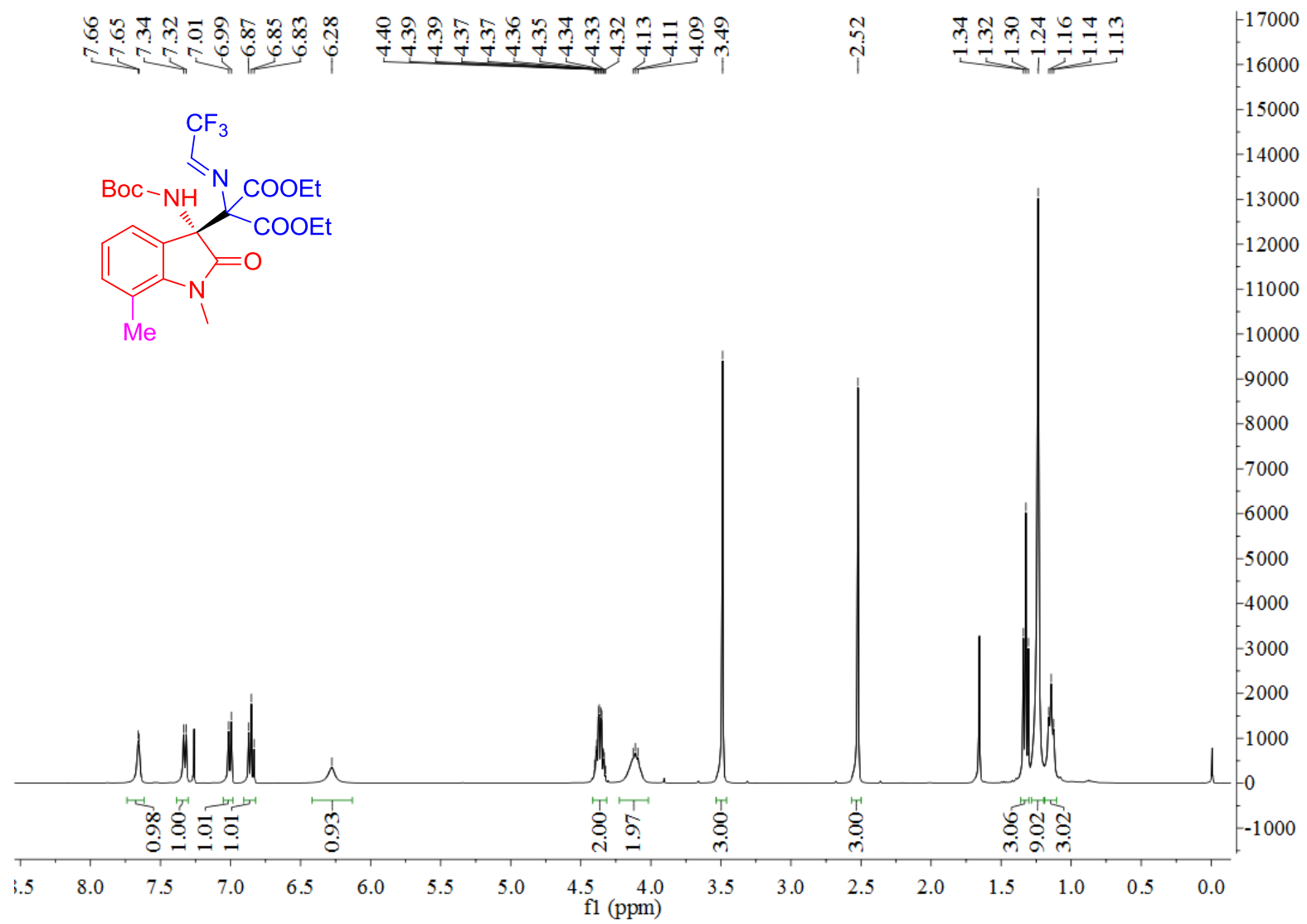


${ }^{13} \mathrm{C}$ NMR of compound $8 \mathrm{j}$ (101 $\mathrm{MHz}$ in $\mathrm{CDCl}_{3}$ )

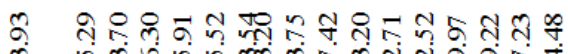

ค
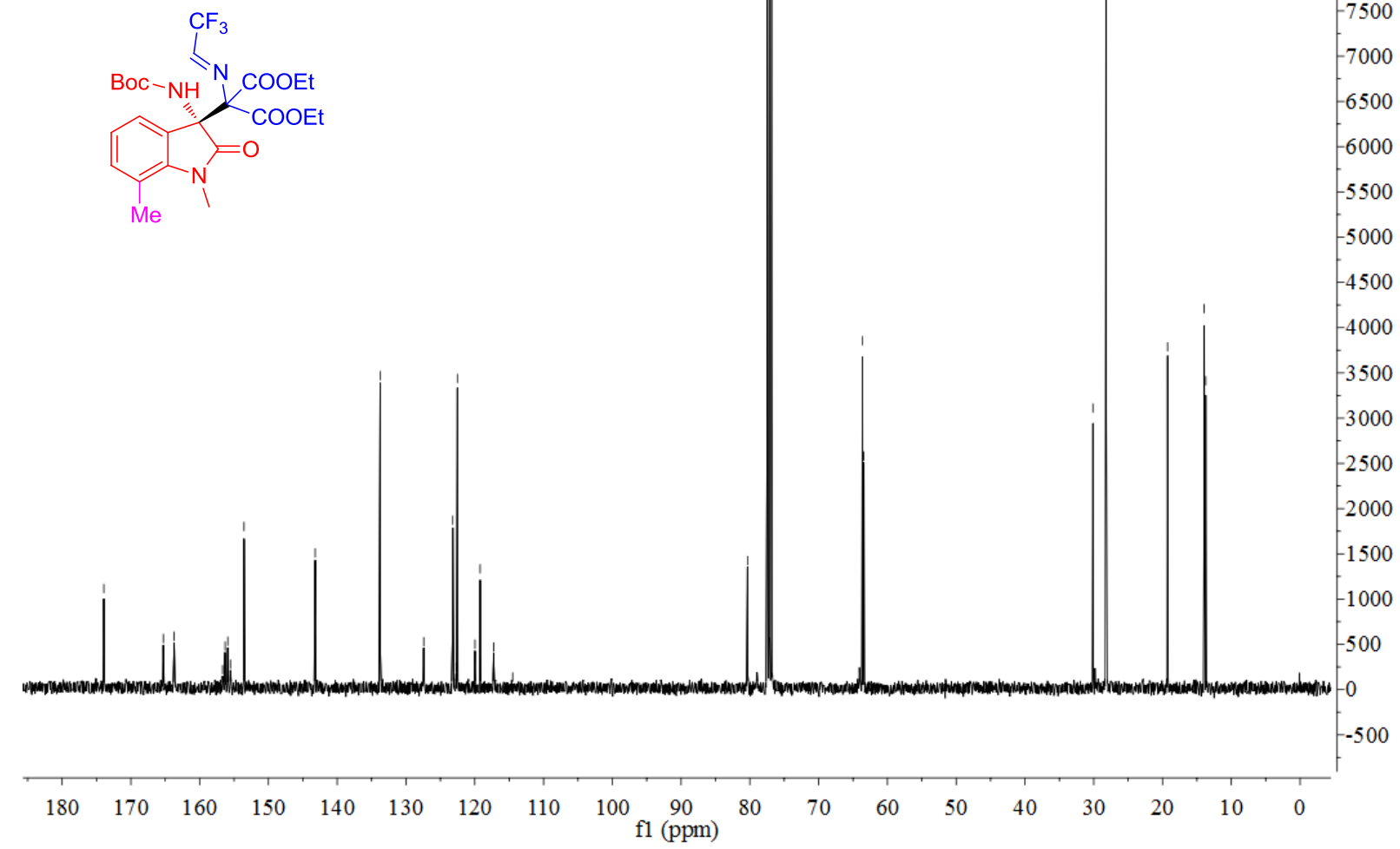

${ }^{19} \mathrm{~F}$ NMR of compound 8j (376 $\mathrm{MHz}$ in $\mathrm{CDCl}_{3}$ )

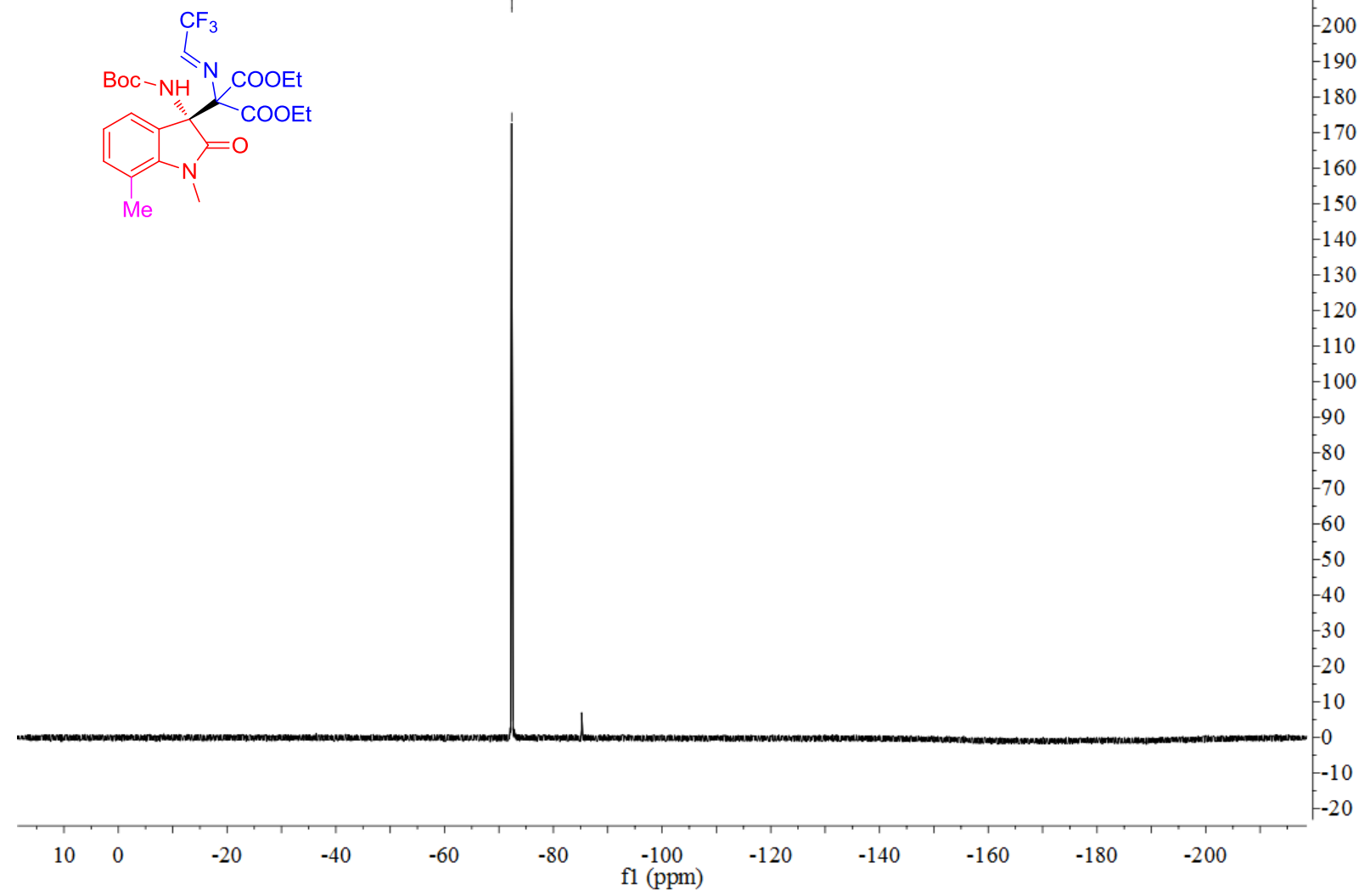


${ }^{1} \mathrm{H}$ NMR of compound $8 \mathrm{~K}$ (400 $\mathrm{MHz}$ in $\mathrm{CDCl}_{3}$ )

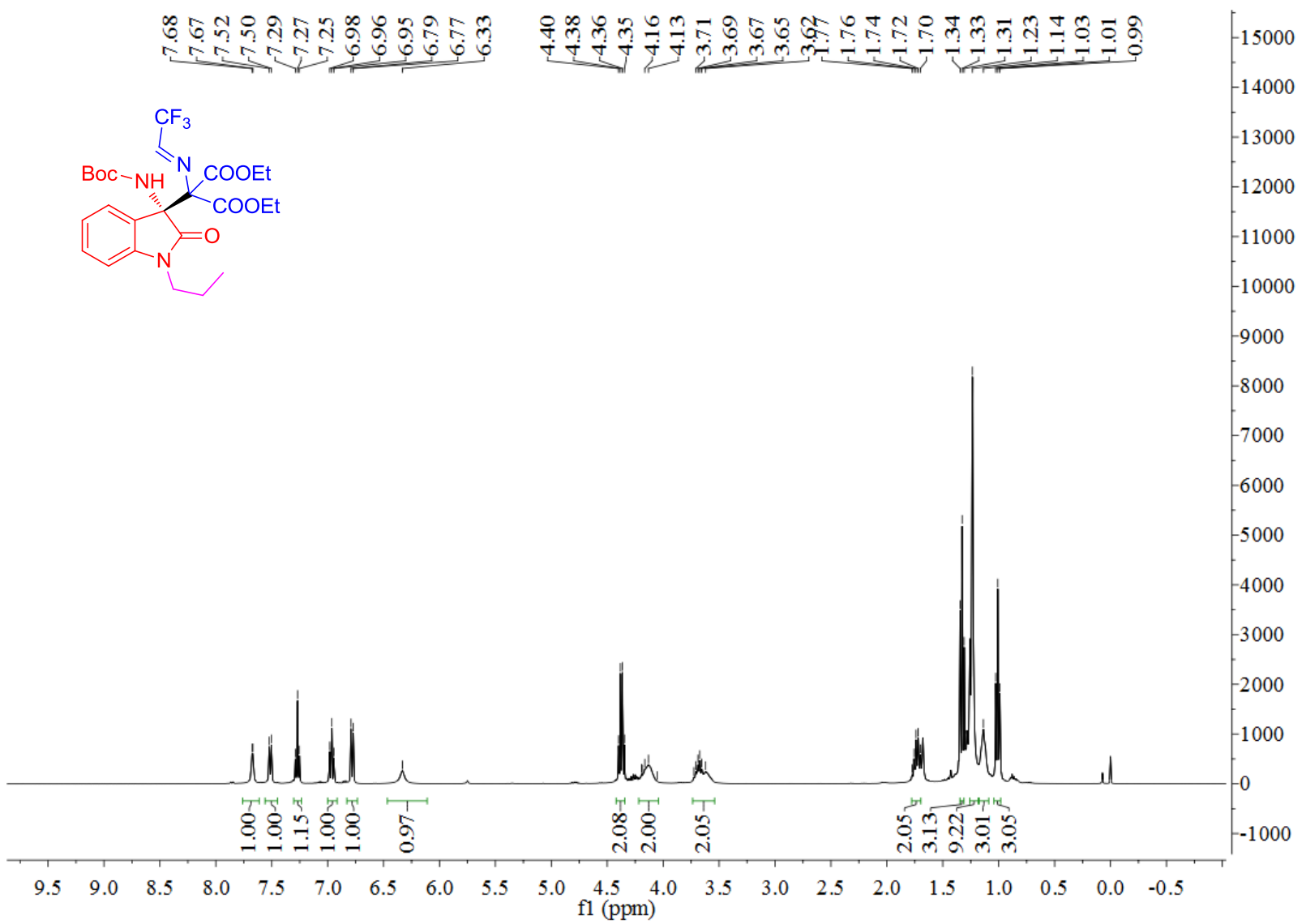

${ }^{13} \mathrm{C}$ NMR of compound 8k (101 $\mathrm{MHz}$ in $\mathrm{CDCl}_{3}$ )

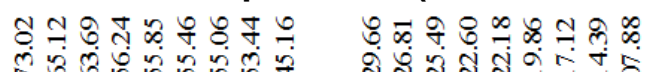

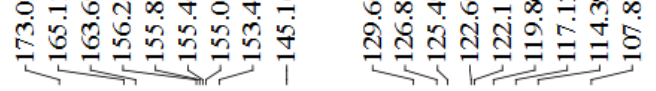

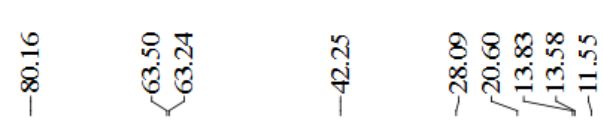

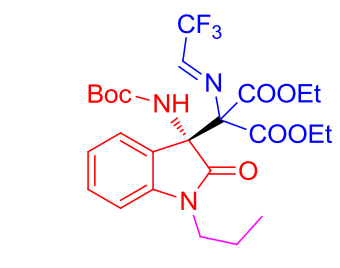

180

170

160

150

130

120

10

fl (ppm)

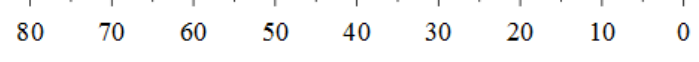


${ }^{19} \mathrm{~F}$ NMR of compound 8k (376 $\mathrm{MHz}$ in $\mathrm{CDCl}_{3}$ )

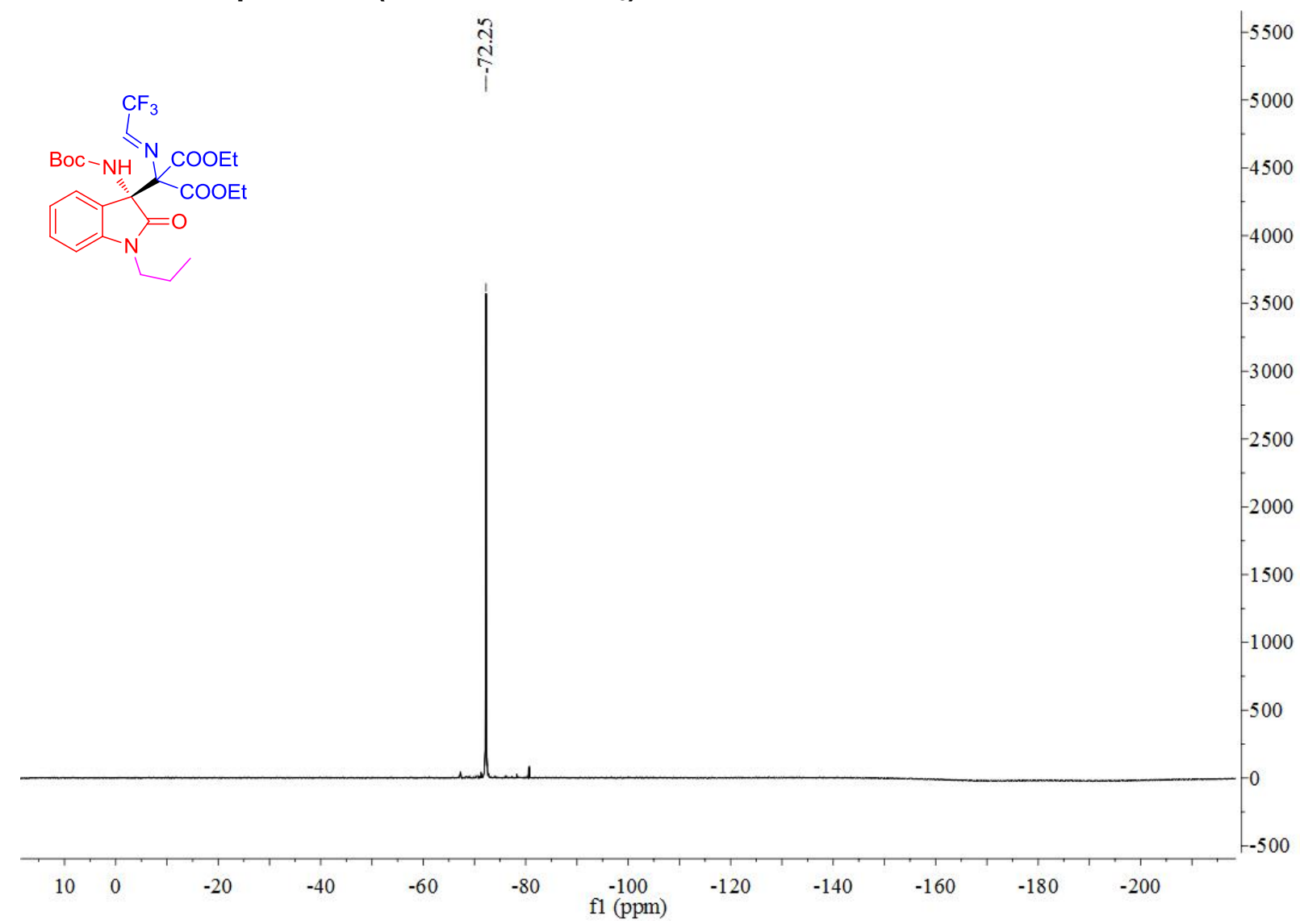

${ }^{1} \mathrm{H}$ NMR of compound $8 \mathrm{I}$ (400 $\mathrm{MHz}$ in $\mathrm{CDCl}_{3}$ )

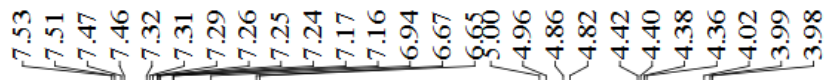

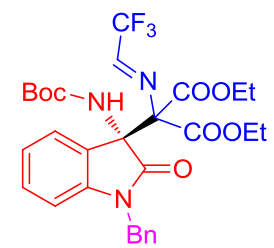

(1)

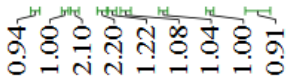

8.0

$\begin{array}{lll}7.5 & 7.0 & 6.5\end{array}$

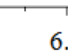

$\begin{array}{lll}6.0 & 5.5 & 5.0\end{array}$

สั)

\section{ले ले लै ते}

=T 
${ }^{13} \mathrm{C}$ NMR of compound $8 \mathrm{I}$ (101 $\mathrm{MHz}$ in $\mathrm{CDCl}_{3}$ )

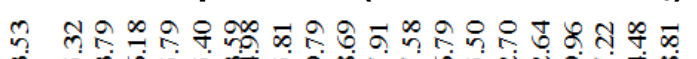

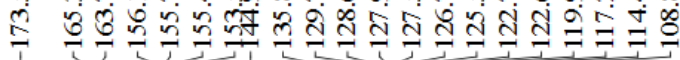

ले केष<smiles>CCOC(=O)N[C@@]1(C(C)(N=CC(F)(F)F)C(=O)OCC)C(=O)Nc2ccccc21</smiles>

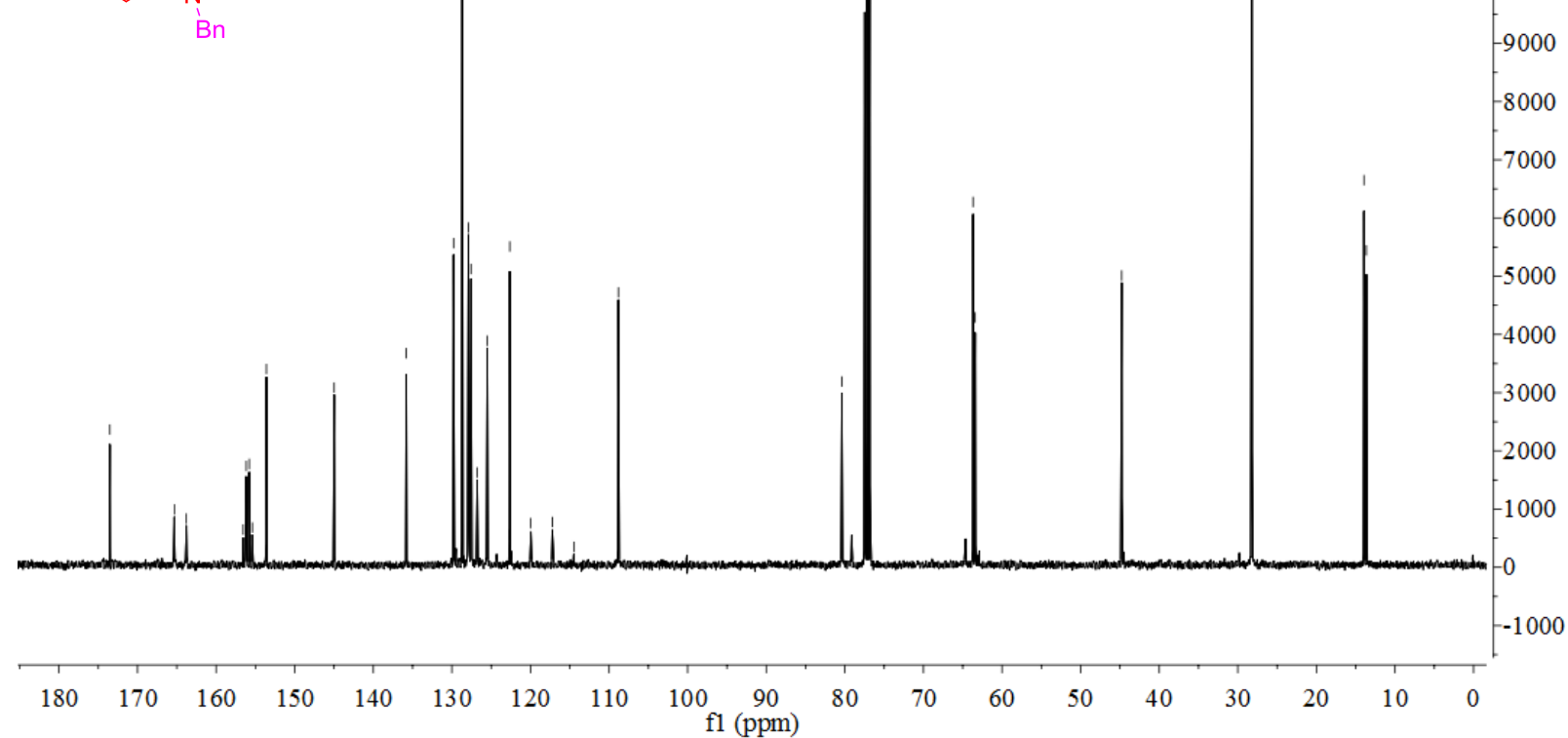

${ }^{19} \mathrm{~F} \mathrm{NMR}$ of compound $8 \mathrm{I}\left(376 \mathrm{MHz}\right.$ in $\mathrm{CDCl}_{3}$ )

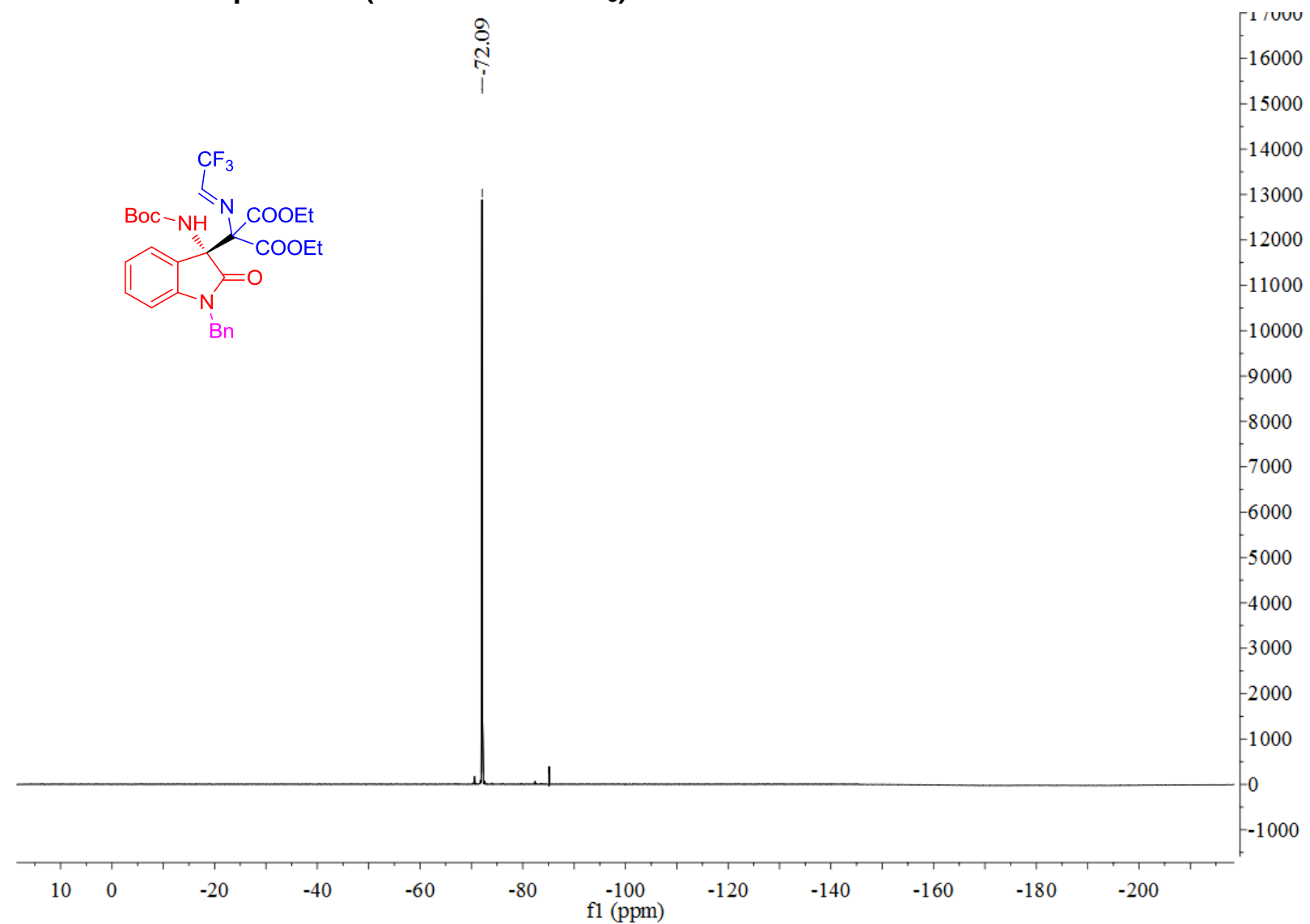




\section{${ }^{1} \mathrm{H}$ NMR of compound $8 \mathrm{~m}\left(400 \mathrm{MHz}\right.$ in $\left.\mathrm{CDCl}_{3}\right)$}

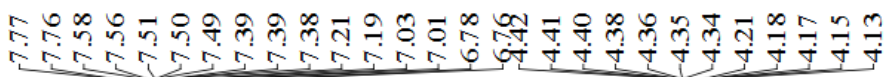
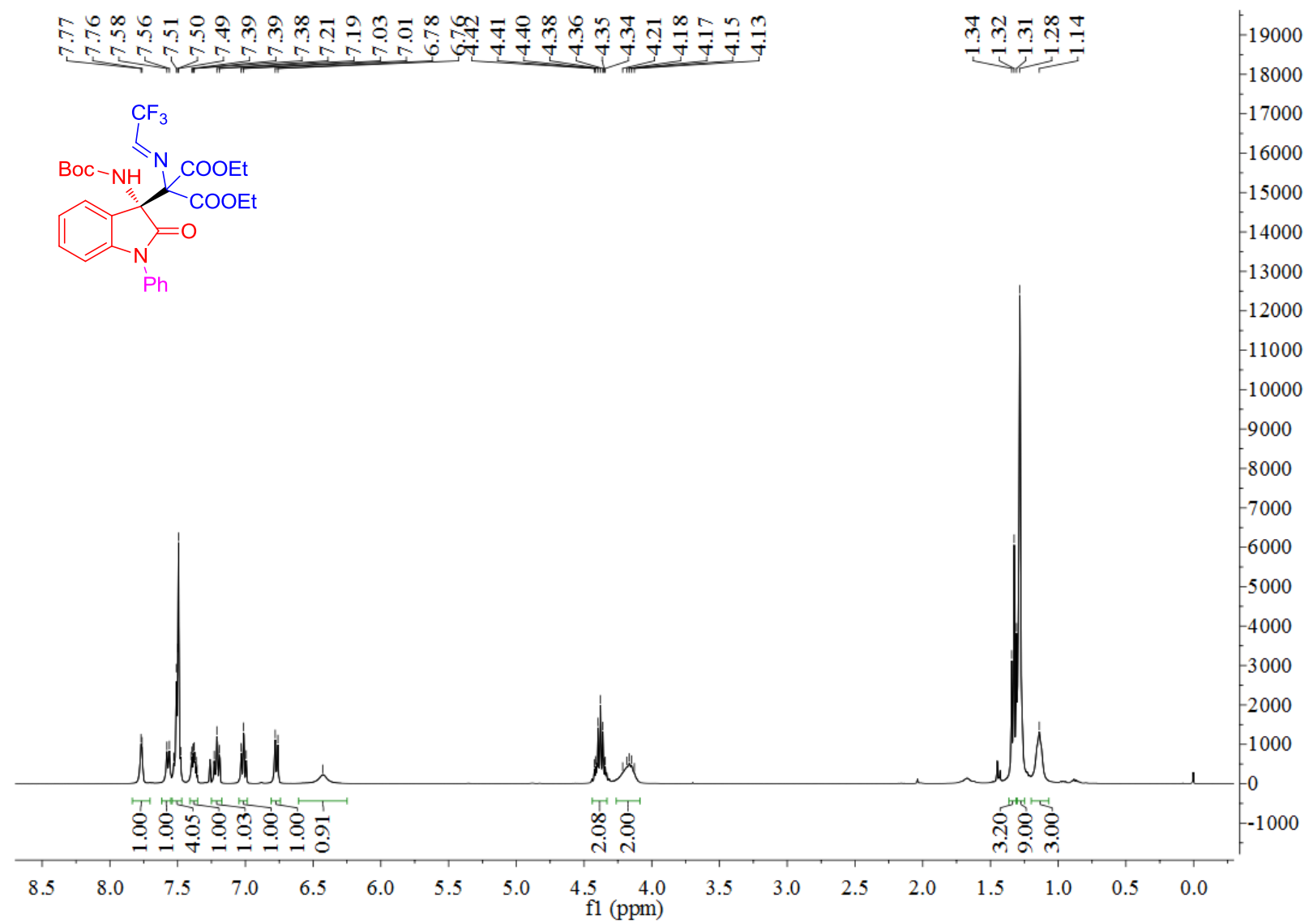

\section{${ }^{13} \mathrm{C}$ NMR of compound $8 \mathrm{~m}$ (101 $\mathrm{MHz}$ in $\mathrm{CDCl}_{3}$ )}

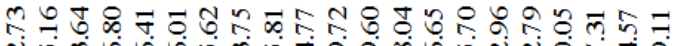

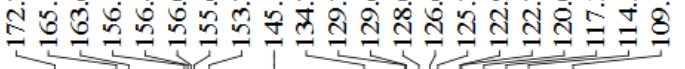

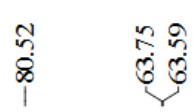
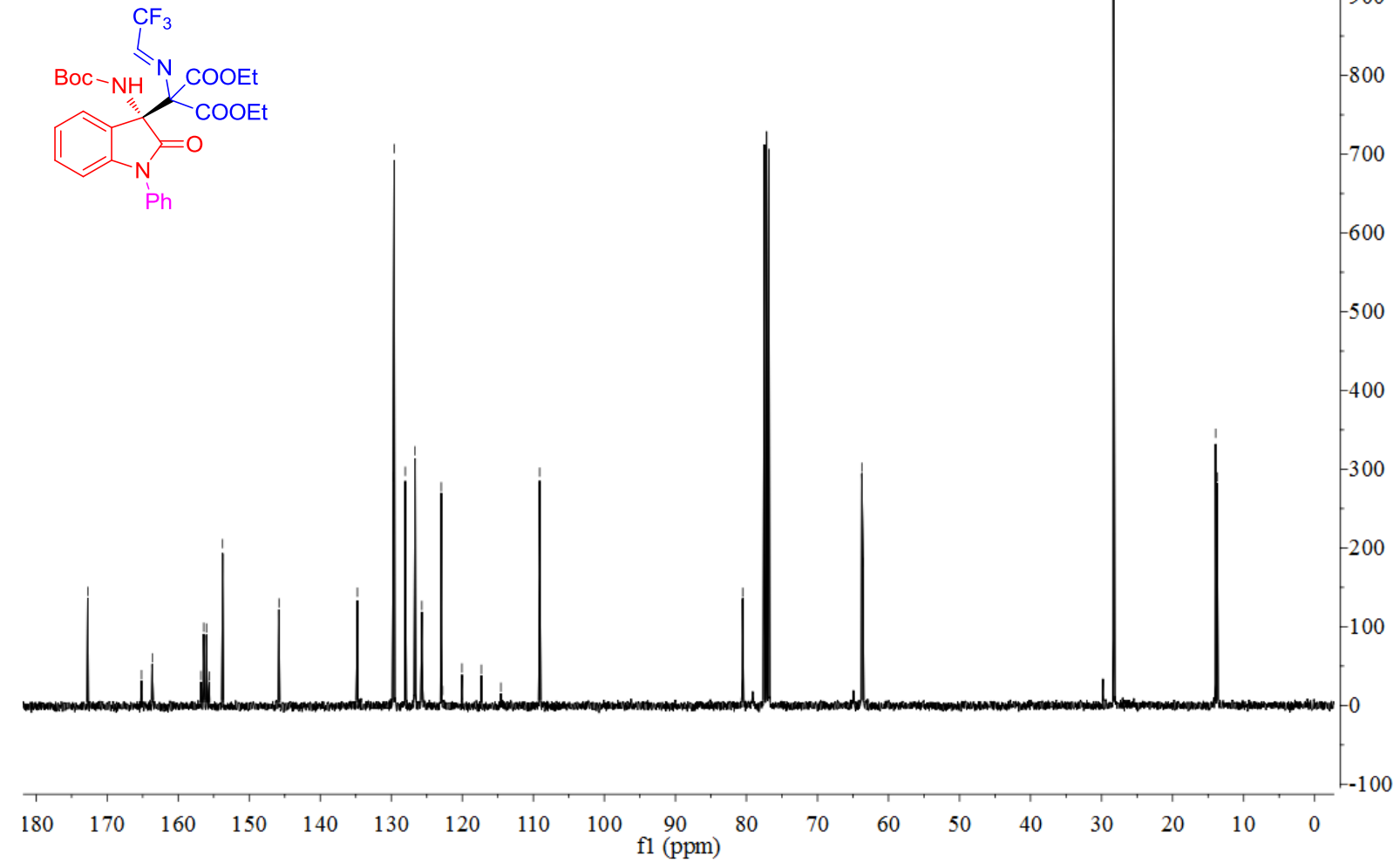
${ }^{19} \mathrm{~F}$ NMR of compound $8 \mathrm{~m}$ (376 $\mathrm{MHz}$ in $\mathrm{CDCl}_{3}$ )

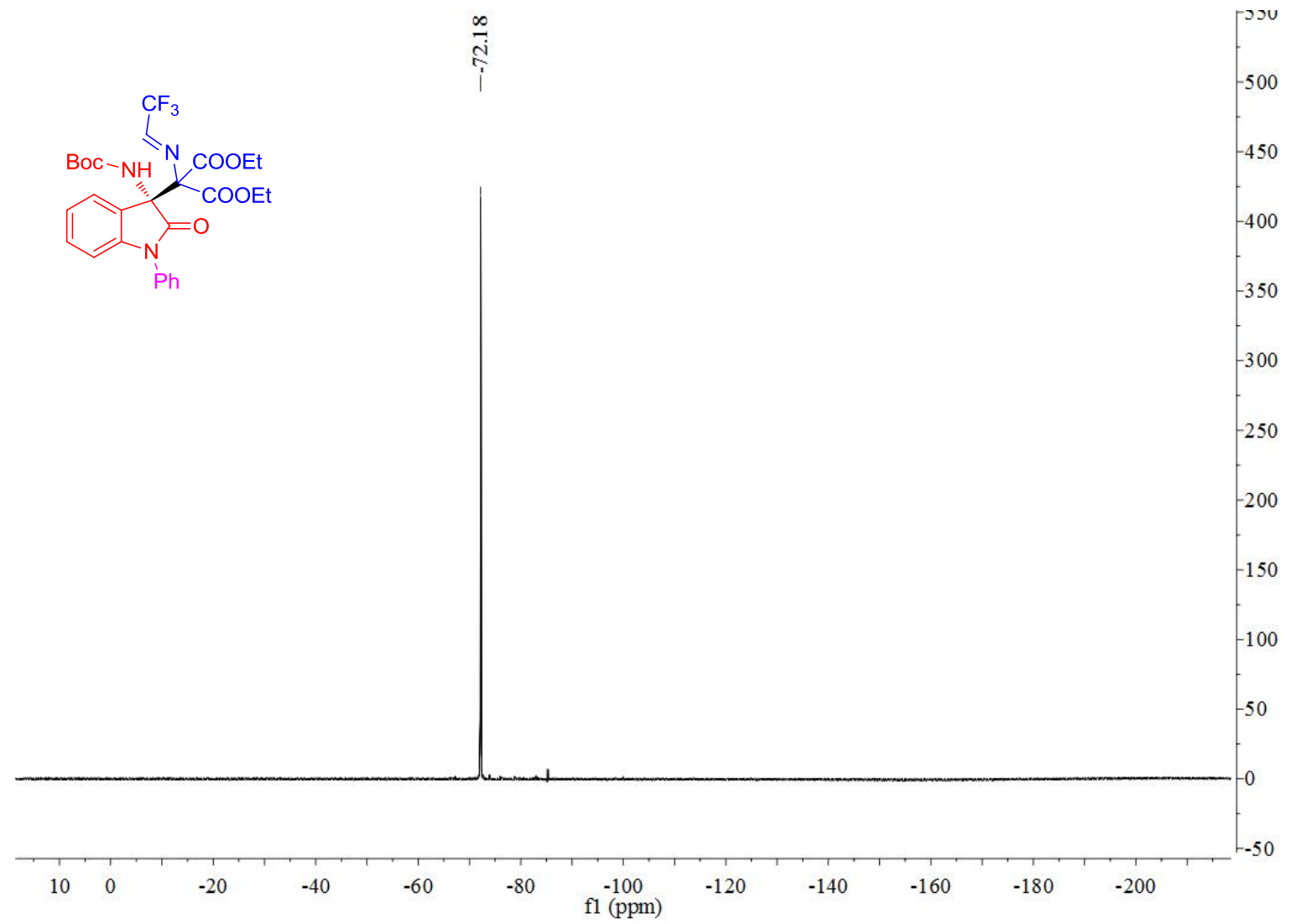

${ }^{1} \mathrm{H}$ NMR of compound 11 (400 $\mathrm{MHz}$ in $\mathrm{CDCl}_{3}$ )

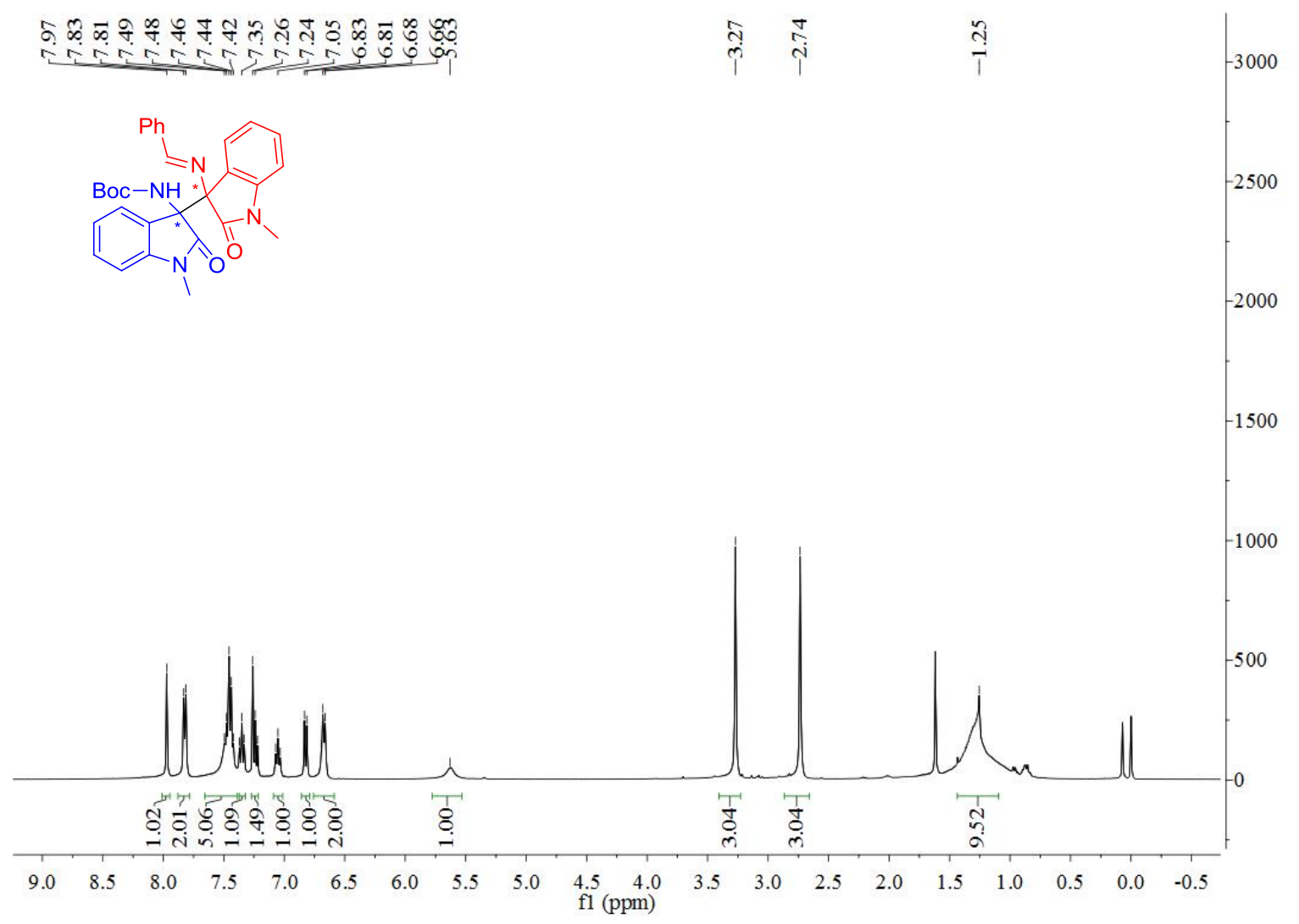


${ }^{13} \mathrm{C}$ NMR of compound $11\left(101 \mathrm{MHz}\right.$ in $\mathrm{CDCl}_{3}$ )

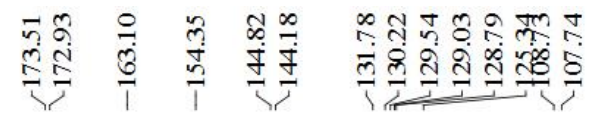

ㄴำ

ชำ

ฝึ่

$-9000$

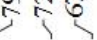

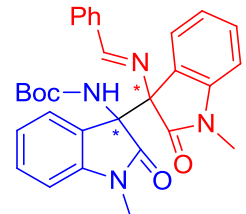

$-7000$

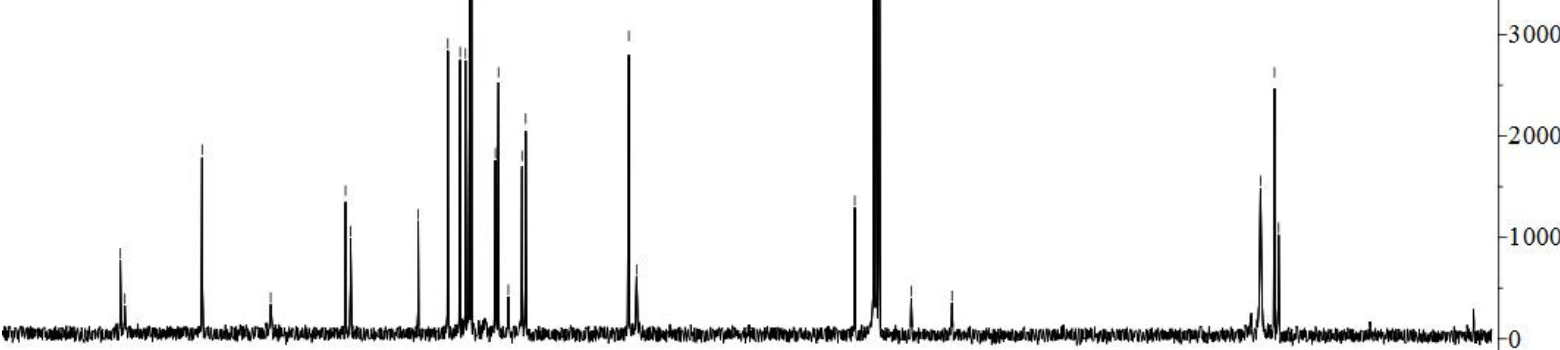

$\begin{array}{llll}180 & 170 \quad 160 & 150 & 140\end{array}$

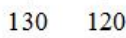

$\begin{array}{rrr}110 & 100 \quad \begin{array}{r}90 \\ \text { f1 (ppm) }\end{array}\end{array}$ 


\section{Copies of HPLC Spectra of Compounds}

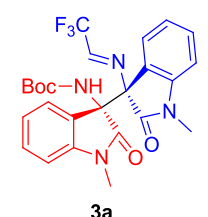

\section{HPLC spectrum of the racemate}

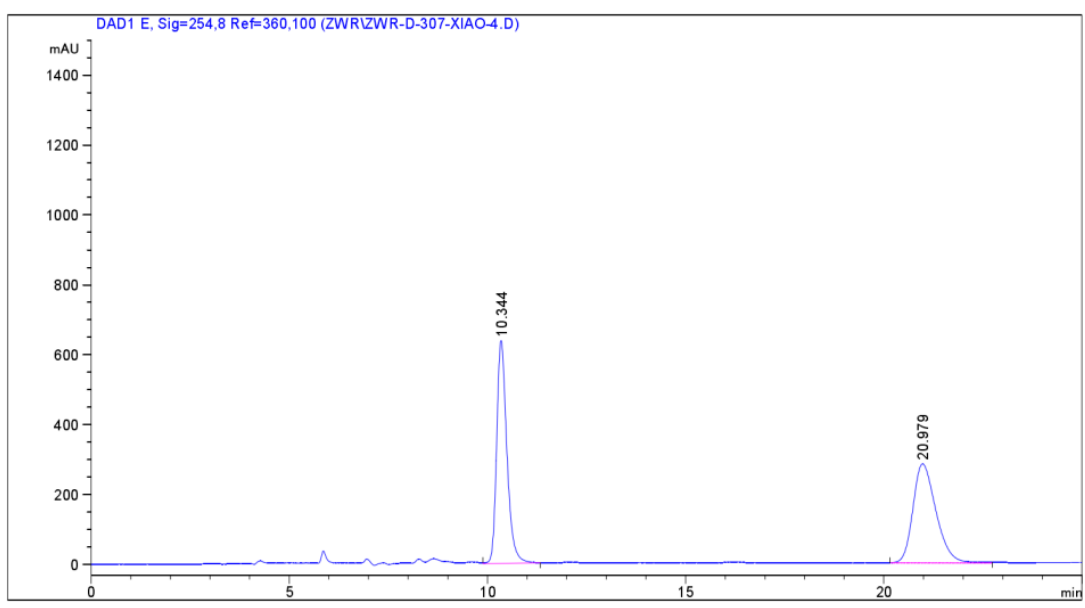

信号 1: DAD1 E， Sig=254,8 Ref=360,100

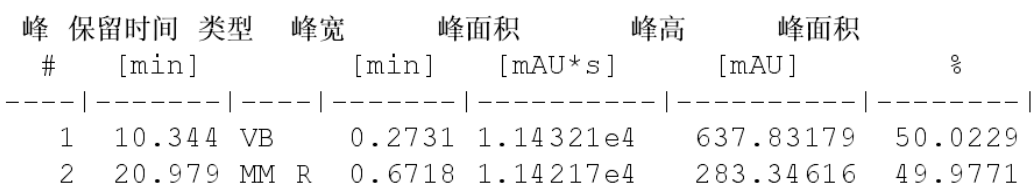

\section{HPLC spectrum of the chiral compound}

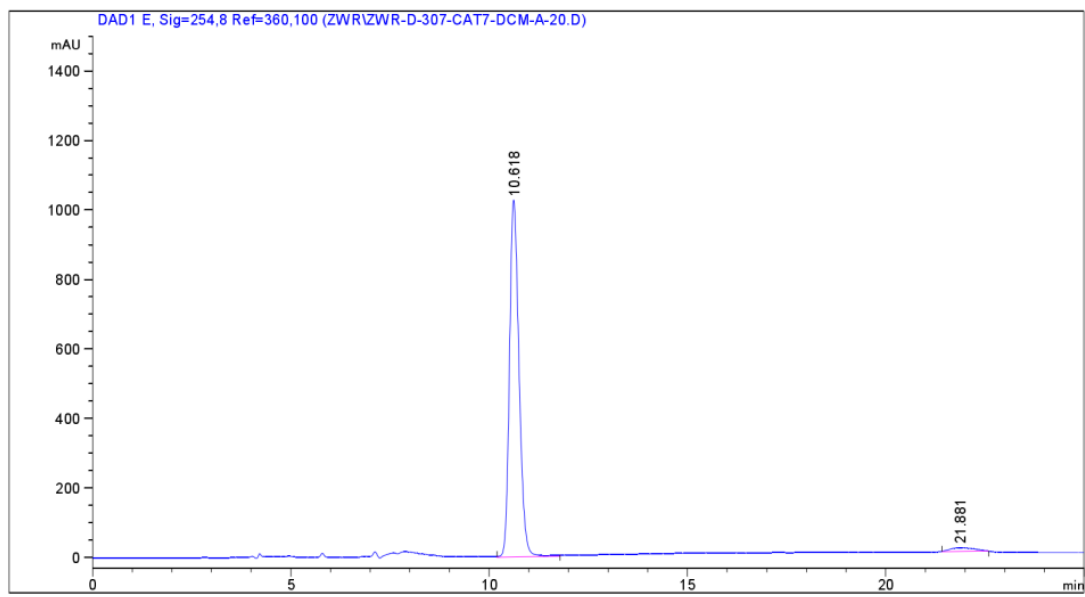

信号 1: DAD1 E， Sig=254,8 Ref=360,100

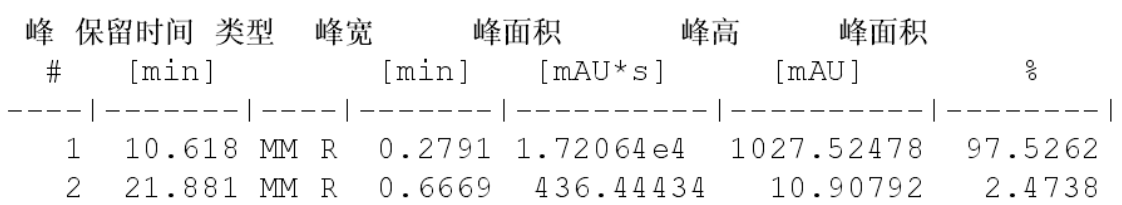




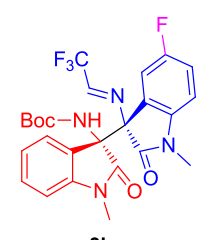

3b

\section{HPLC spectrum of the racemate}

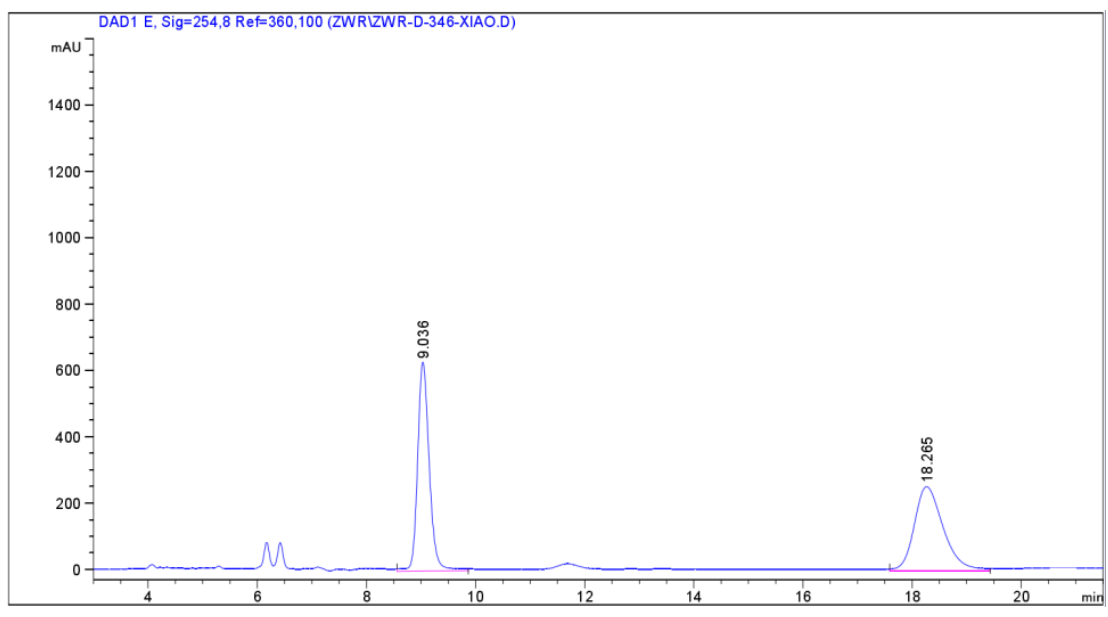

信号 1: DAD1 E， Sig=254,8 Ref=360,100

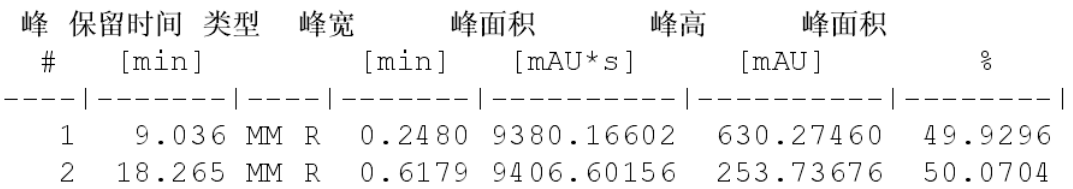

\section{HPLC spectrum of the chiral compound}

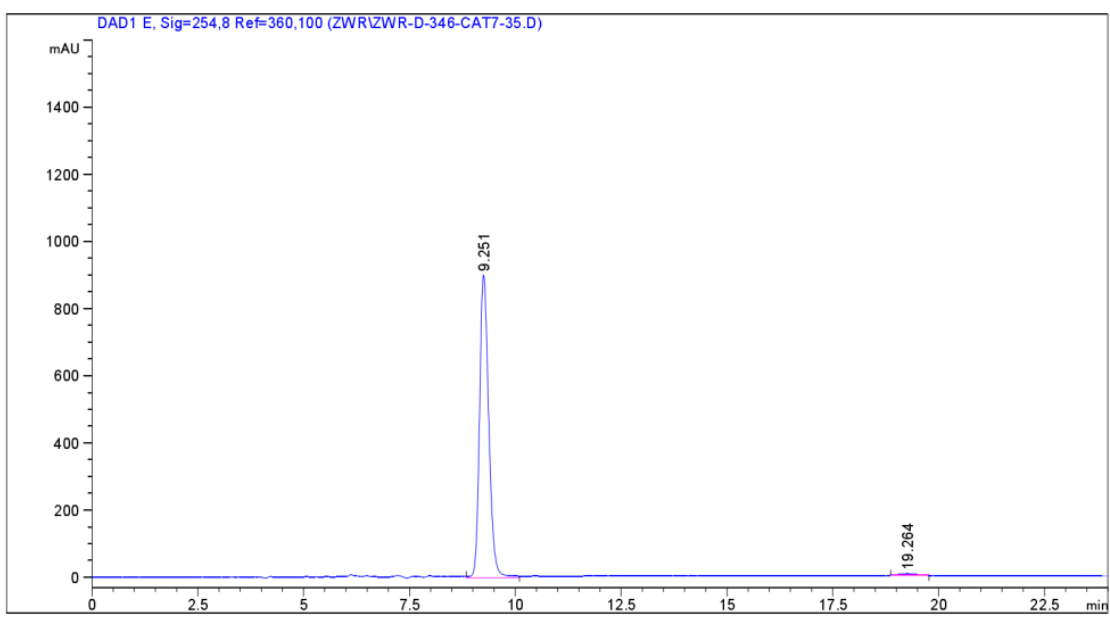

信号 1: DAD1 E， Sig=254,8 $\operatorname{Ref}=360,100$

\begin{tabular}{|c|c|c|c|c|c|c|c|}
\hline 峰 保 & 留时间 类型 & 峰宽 & & 面积 & 峰高 & \multicolumn{2}{|l|}{ 峰面积 } \\
\hline \# & [min $]$ & & [min $]$ & {$\left[\mathrm{mAU}{ }^{\star} \mathrm{s}\right]$} & & [mAU ] & $\%$ \\
\hline 1 & MM & $\mathrm{R}$ & 0.2520 & $1.36365 e$ & & 902.00592 & 99.0245 \\
\hline 2 & $19.264 \mathrm{MM}$ & $\mathrm{R}$ & 0.4769 & 134.330 & & 4.69453 & 0.9755 \\
\hline
\end{tabular}




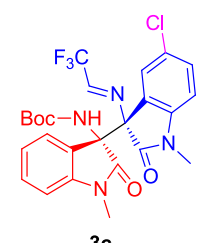

$3 c$

\section{HPLC spectrum of the racemate}

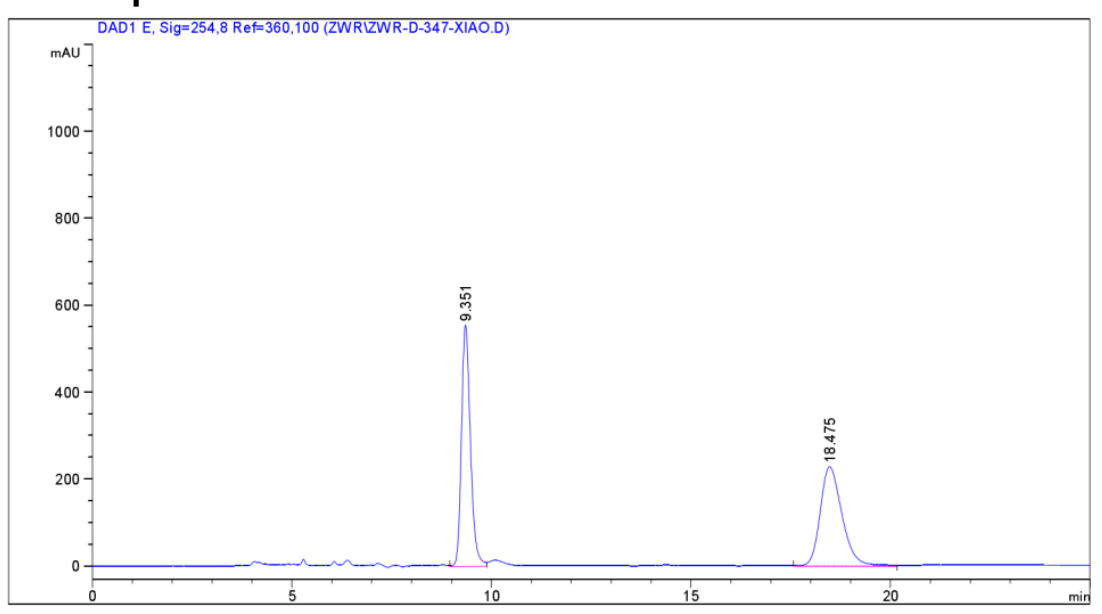

信号 1: DAD1 E， Sig=254, 8 Ref $=360,100$

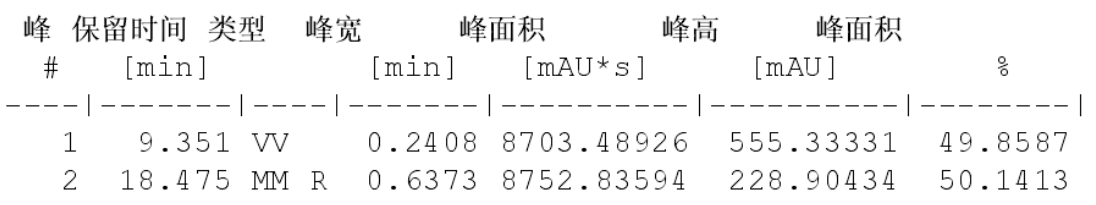

\section{HPLC spectrum of the chiral compound}

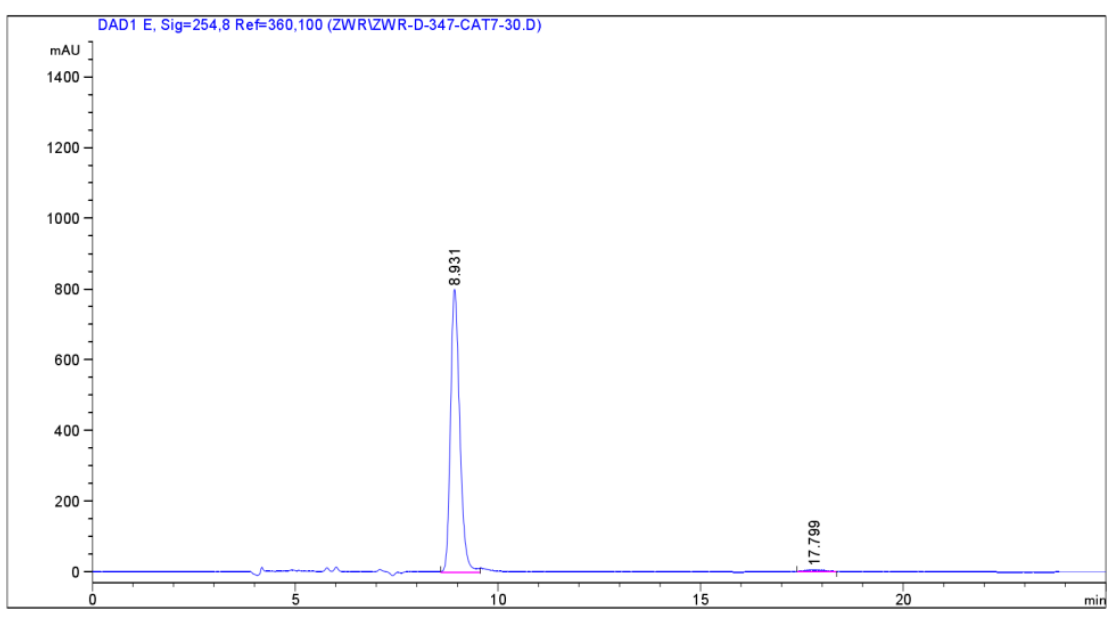

信号 1: DAD1 E， Sig=254,8 Ref $=360,100$

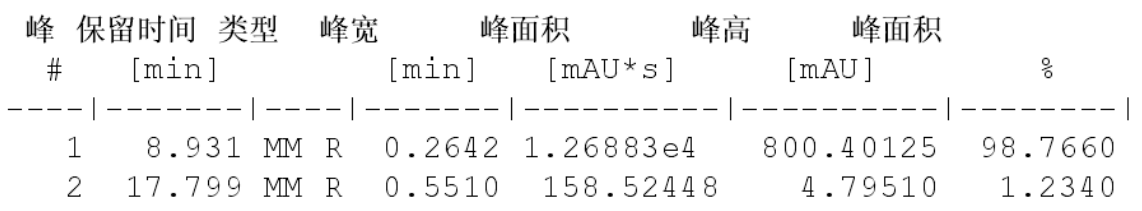




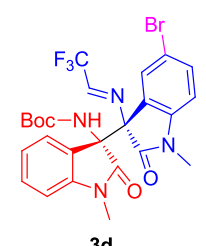

\section{HPLC spectrum of the racemate}

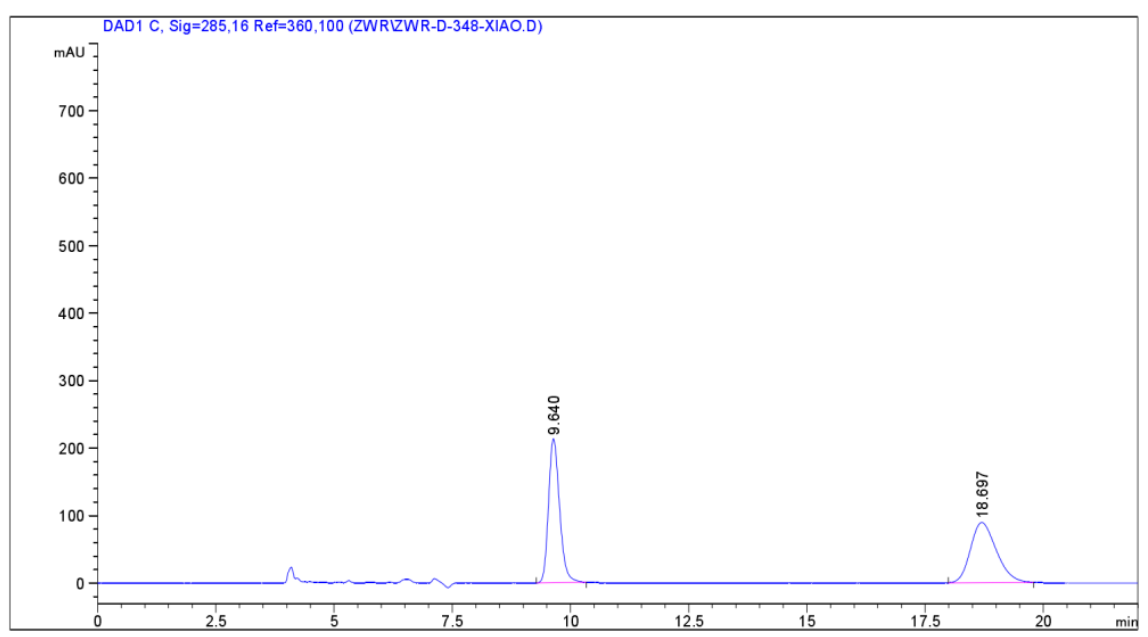

信号 1: DAD1 C, Sig=285,16 Ref=360,100

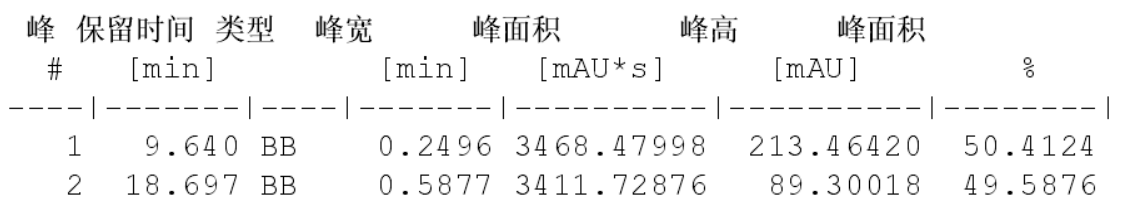

\section{HPLC spectrum of the chiral compound}

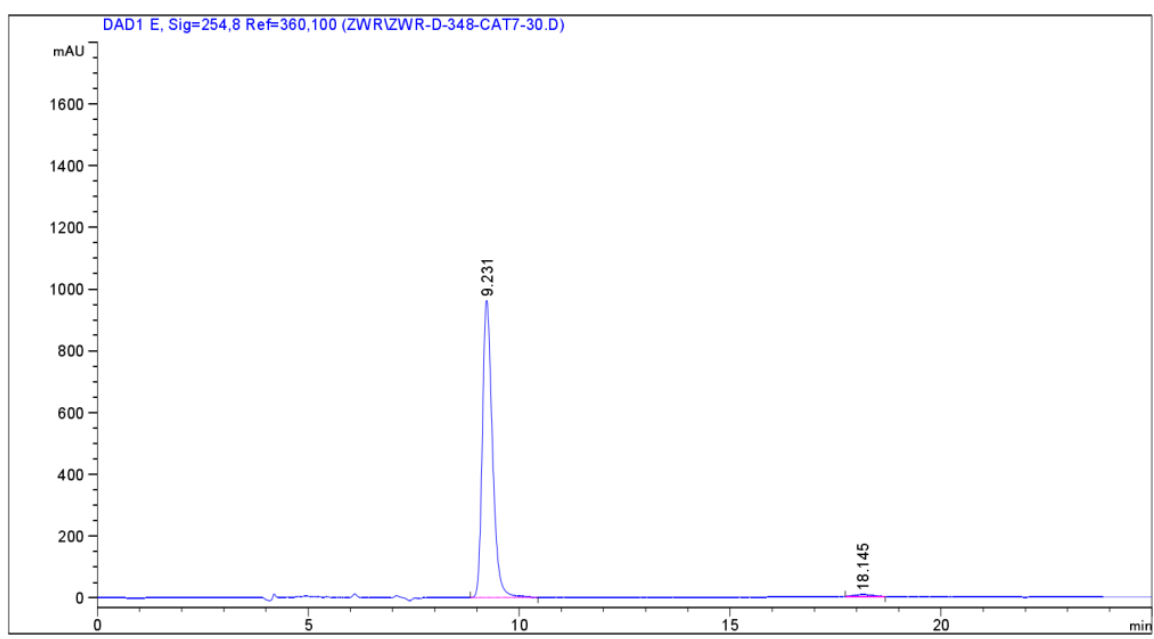

信号 1: DAD1 E, Sig=254,8 Ref=360,100

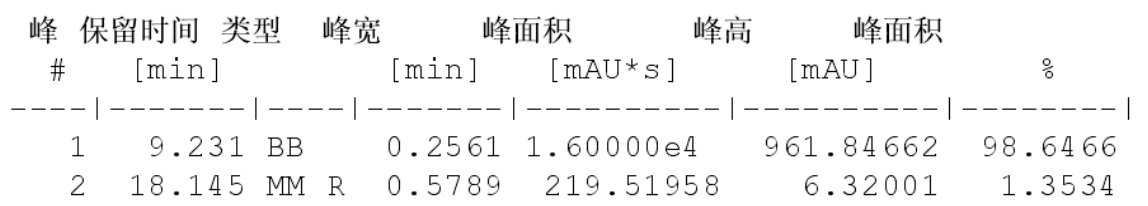




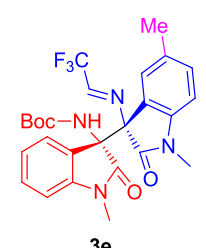

HPLC spectrum of the racemate

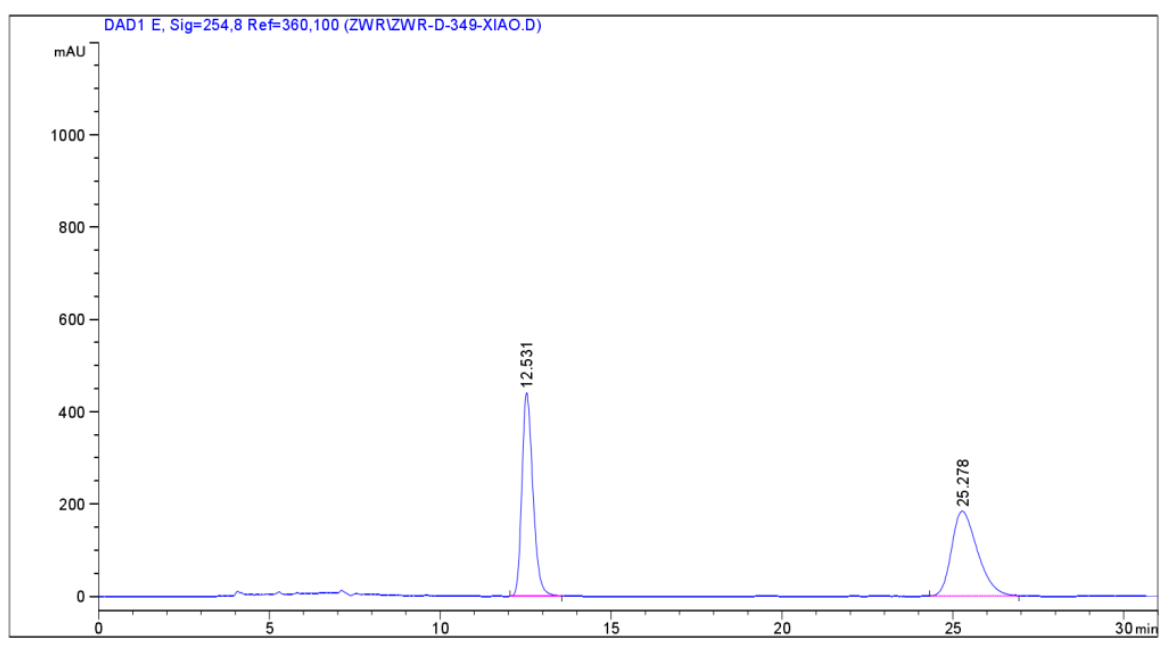

信号 1: DAD1 E， Sig=254,8 Ref=360,100

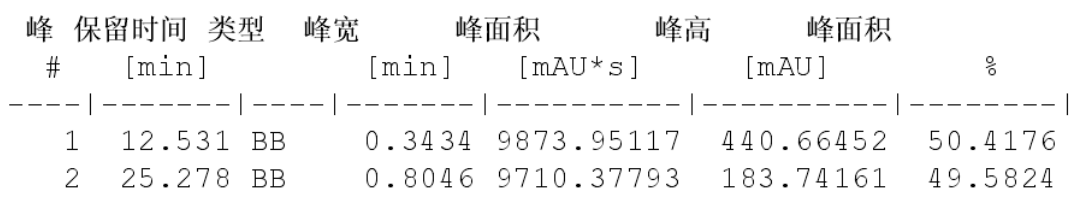

\section{HPLC spectrum of the chiral compound}

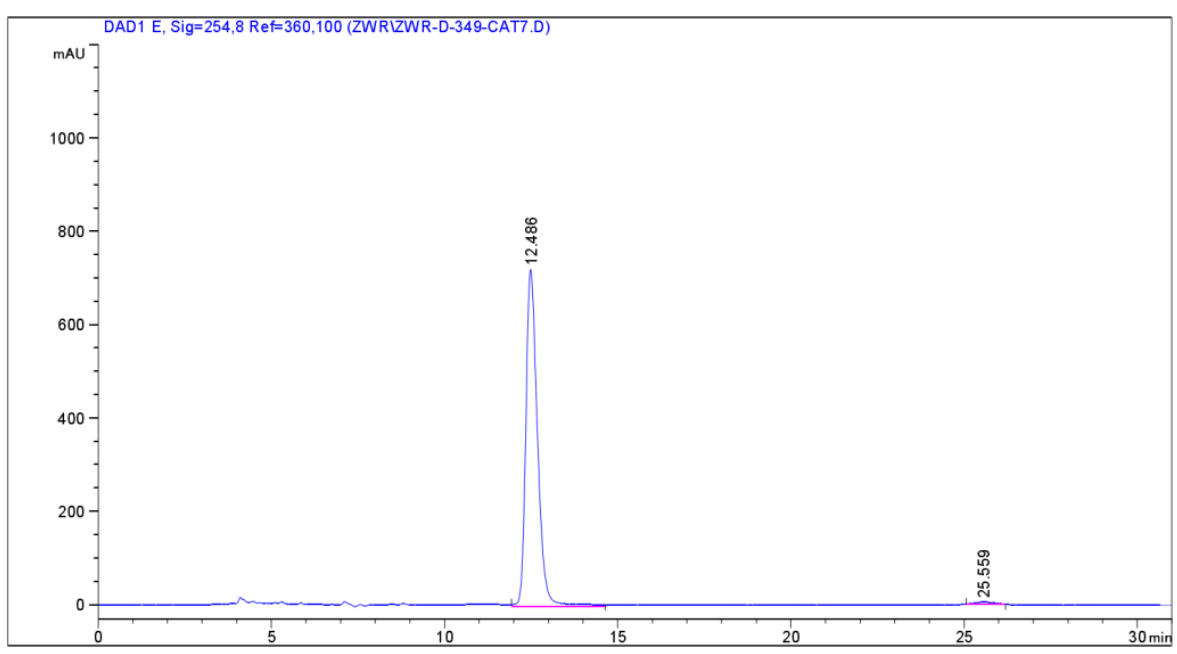

信号 1: DAD1 E, Sig=254, 8 Ref=360,100

\begin{tabular}{|c|c|c|c|c|c|c|c|}
\hline 峰 & 保留时间 类型 & 峰宽 & & 面积 & 峰高 & \multicolumn{2}{|l|}{ 峰面积 } \\
\hline \# & [min $]$ & & {$[\mathrm{min}]$} & {$[\mathrm{mAU} * \mathrm{~S}]$} & & [mAU ] & $\%$ \\
\hline 1 & $86 \mathrm{MN}$ & $\mathrm{R}$ & 0.3929 & $1.70225 \mathrm{e}$ & & 722.08636 & 99.0009 \\
\hline 2 & $25.559 \mathrm{MI}$ & $\mathrm{R}$ & 0.6630 & 171.790 & & 4.31854 & 0.9991 \\
\hline
\end{tabular}




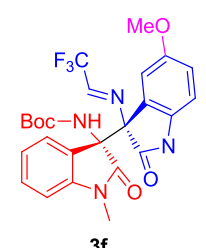

HPLC spectrum of the racemate

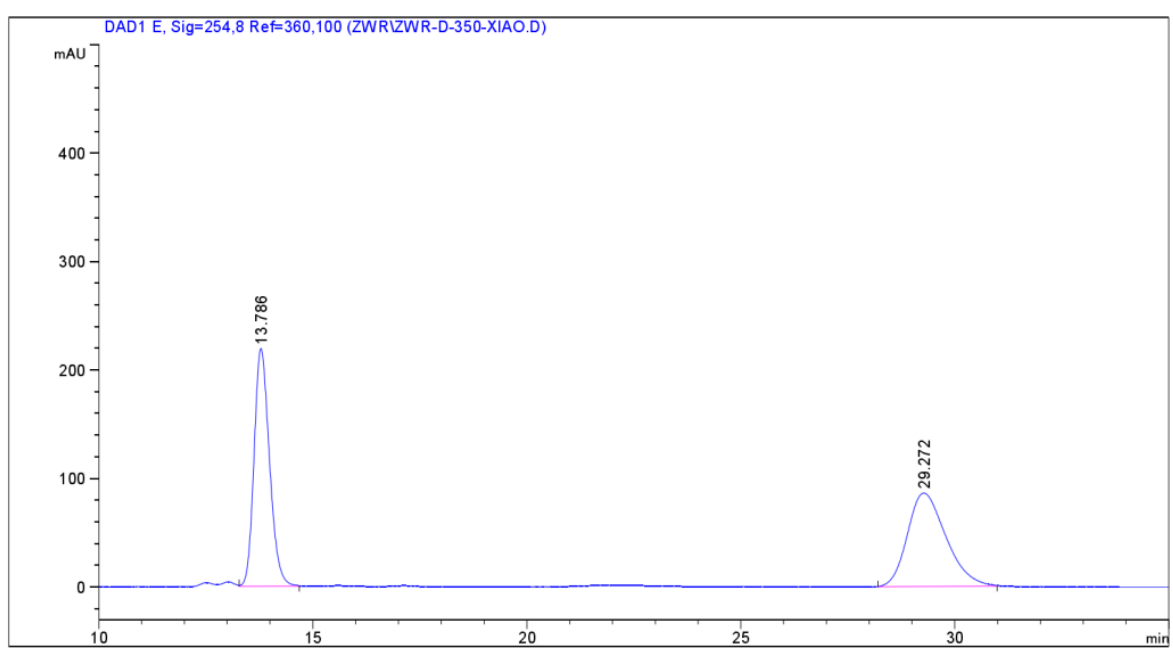

信号 1: DAD1 C, Sig=285,16 Ref=360,100

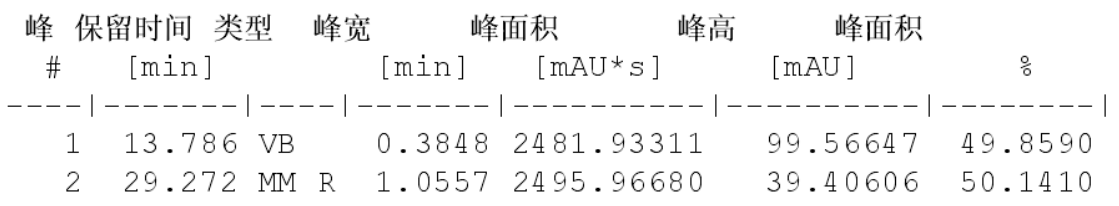

\section{HPLC spectrum of the chiral compound}

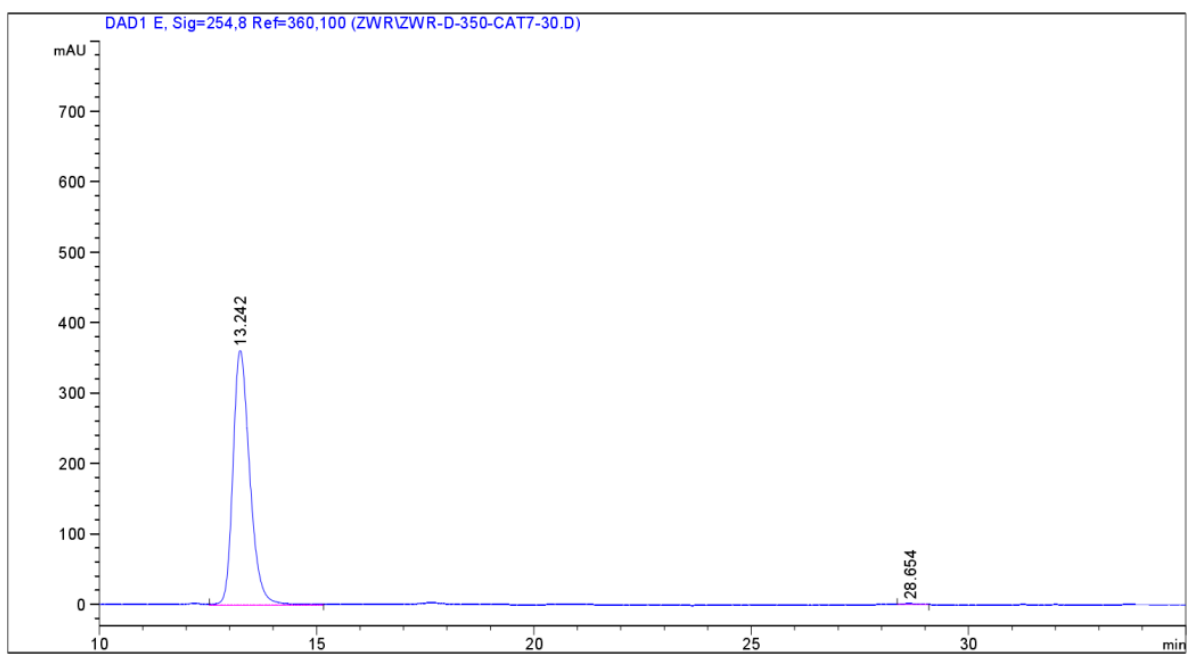

信号 1: DAD1 E， Sig=254,8 Ref=360,100

\begin{tabular}{|c|c|c|c|c|c|c|c|}
\hline 峰 & 留时间 类型 & 峰宽 & & 面积 & 峰高 & \multicolumn{2}{|l|}{ 峰面积 } \\
\hline \# & [min] & & [min] & {$[\mathrm{mAU} * \mathrm{~s}]$} & & [mAU ] & \% \\
\hline & --- & & ------ & ------ & & -------- & ------- \\
\hline 1 & 13.242 & $\mathrm{R}$ & 0.4510 & 9801.512 & 70 & 362.19843 & 99.6842 \\
\hline 2 & $28.654 \mathrm{M}$ & $R$ & 0.4297 & 31.050 & 50 & 1.20424 & 0.3158 \\
\hline
\end{tabular}




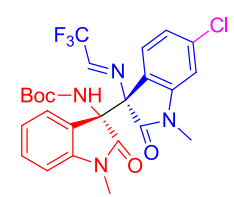

$3 g$

\section{HPLC spectrum of the racemate}

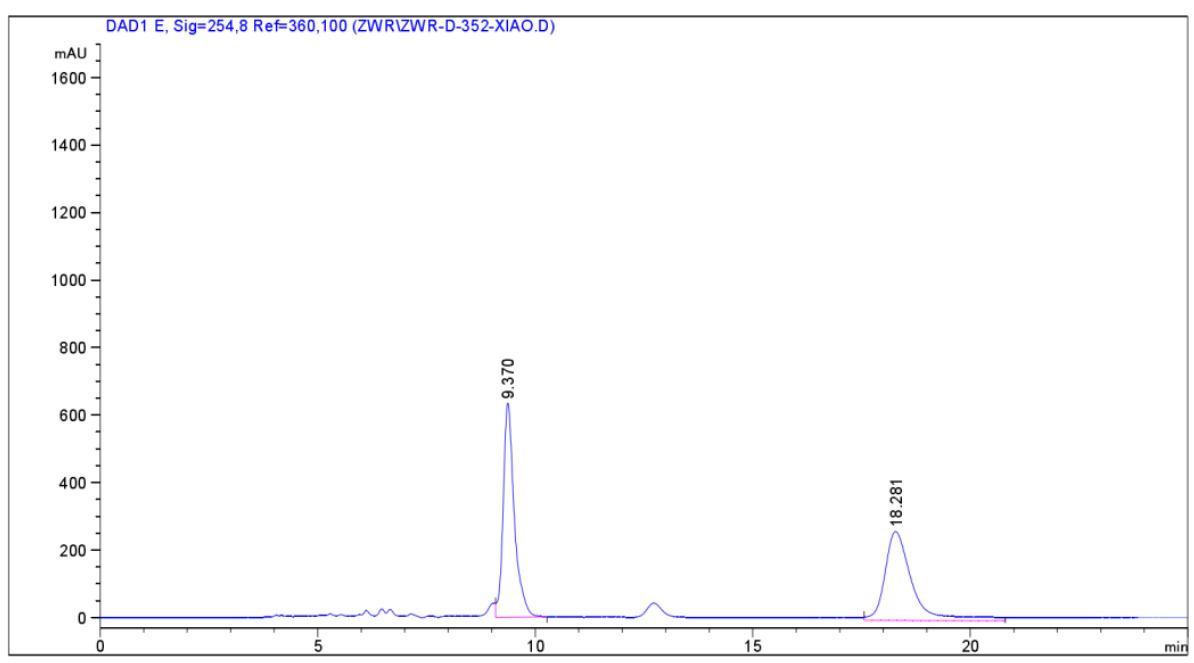

信号 1: DAD1 E， Sig=254,8 Ref=360,100

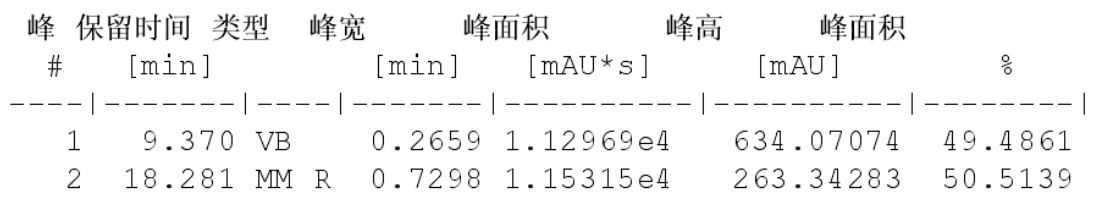

\section{HPLC spectrum of the chiral compound}

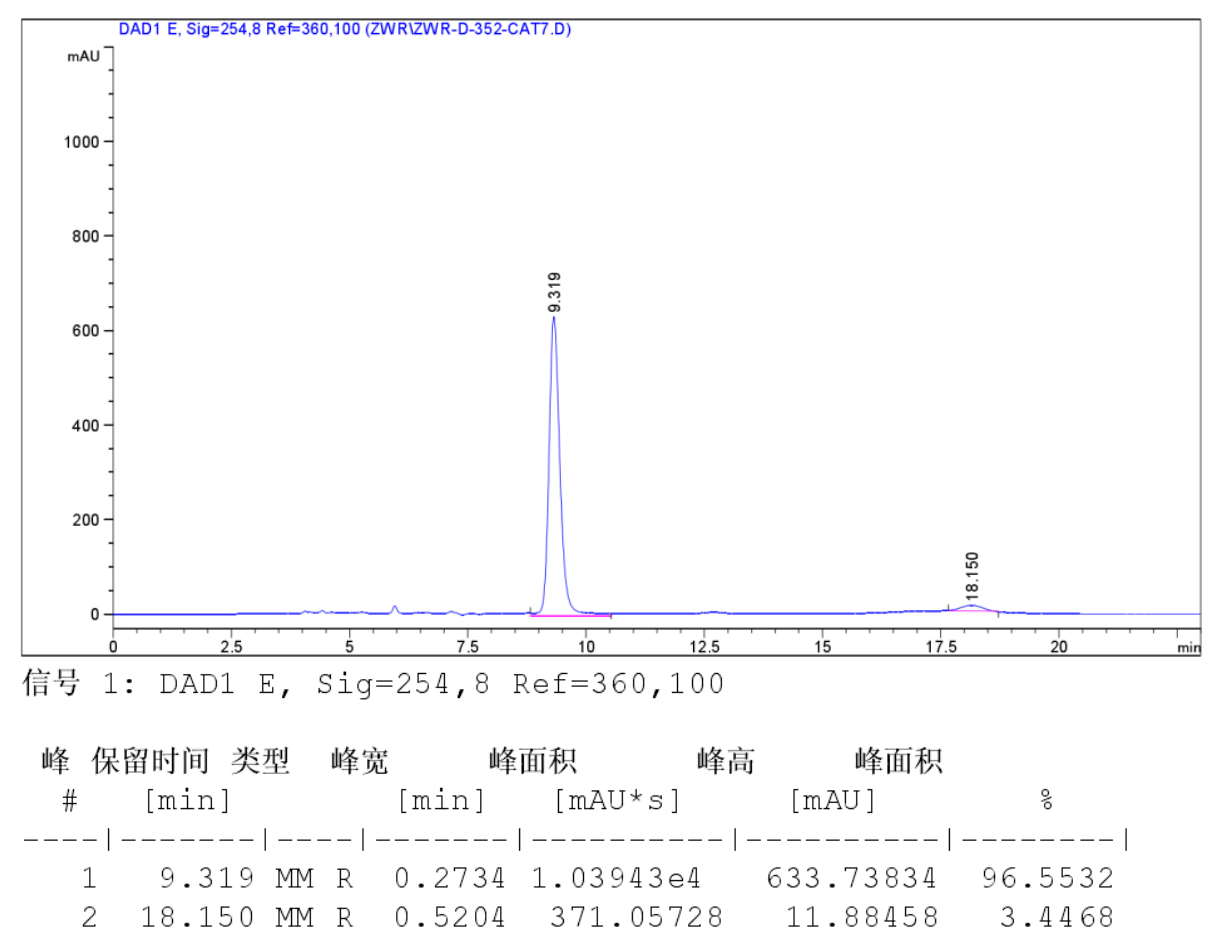




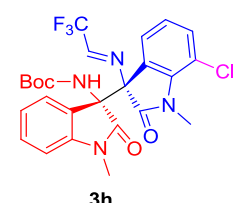

\section{HPLC spectrum of the racemate}

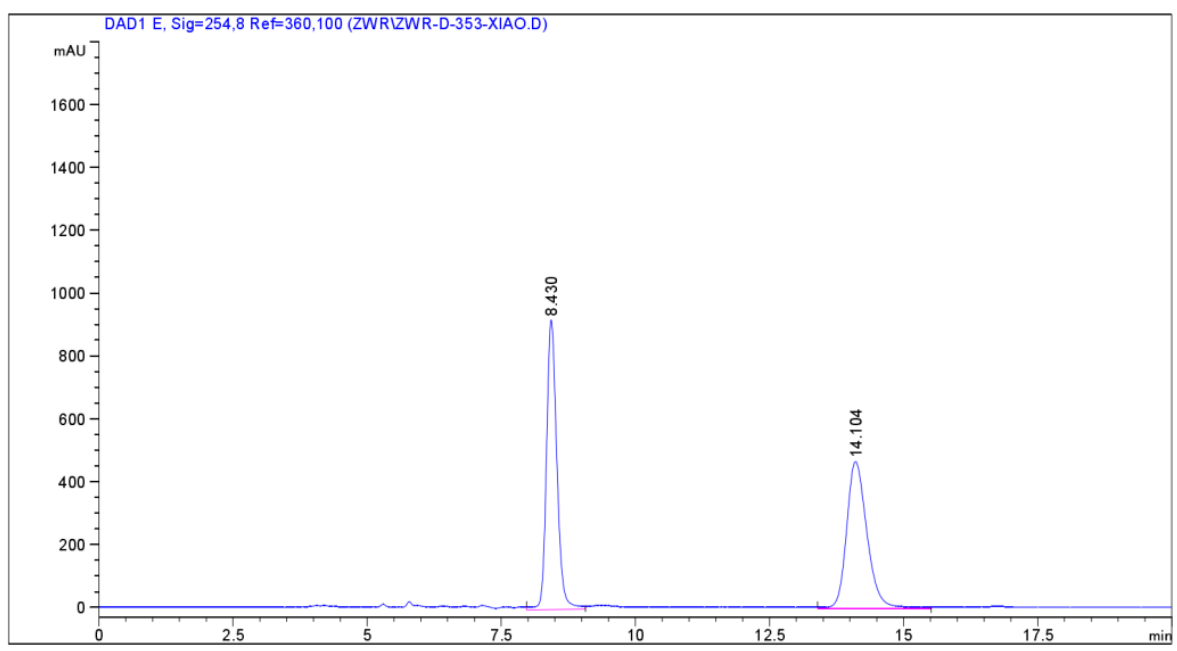

信号 1: DAD1 E, Sig=254,8 Ref=360,100

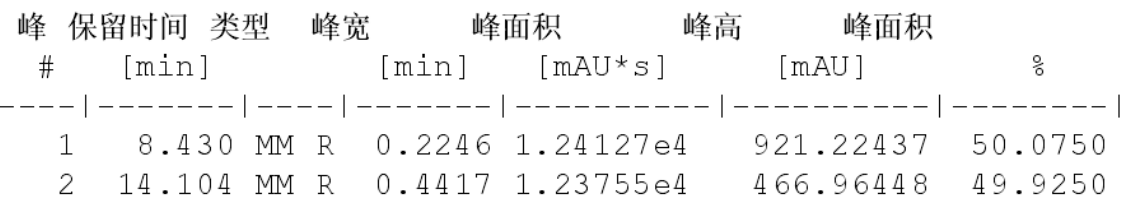

\section{HPLC spectrum of the chiral compound}

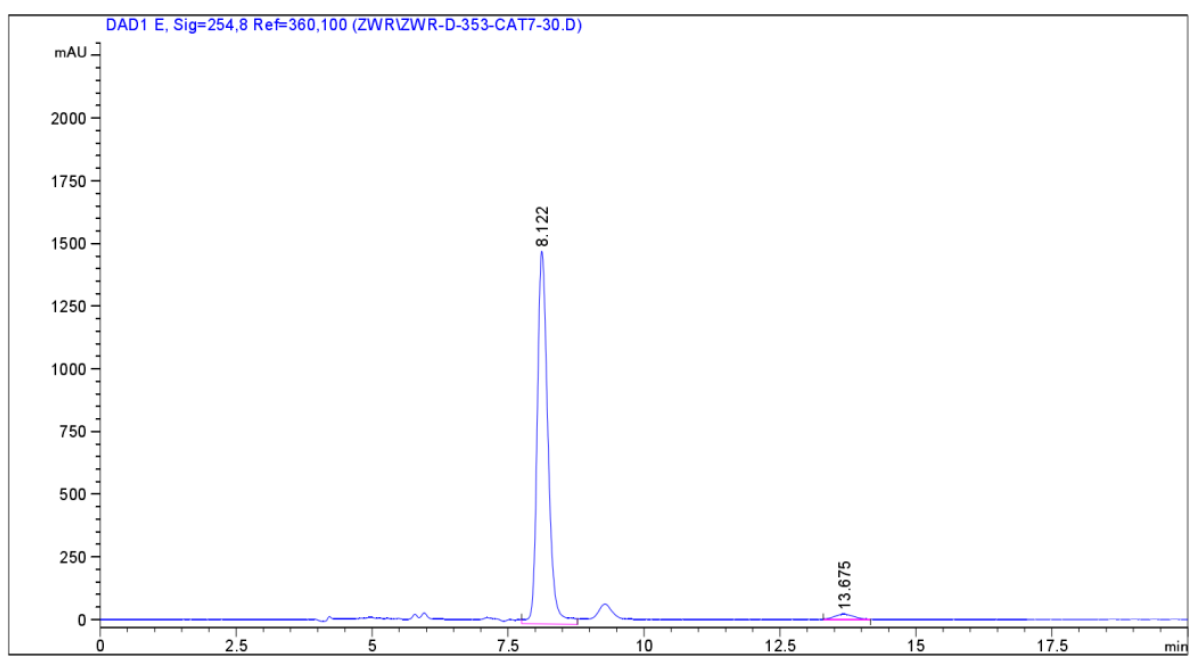

信号 1: DAD1 E， Sig=254,8 Ref=360,100

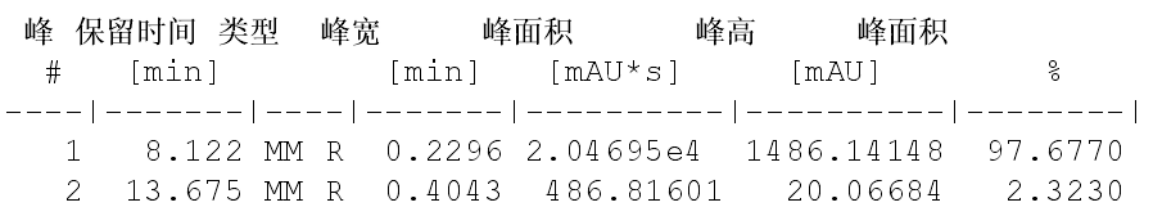




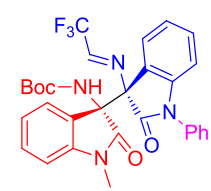

$3 \mathbf{i}$

HPLC spectrum of the racemate

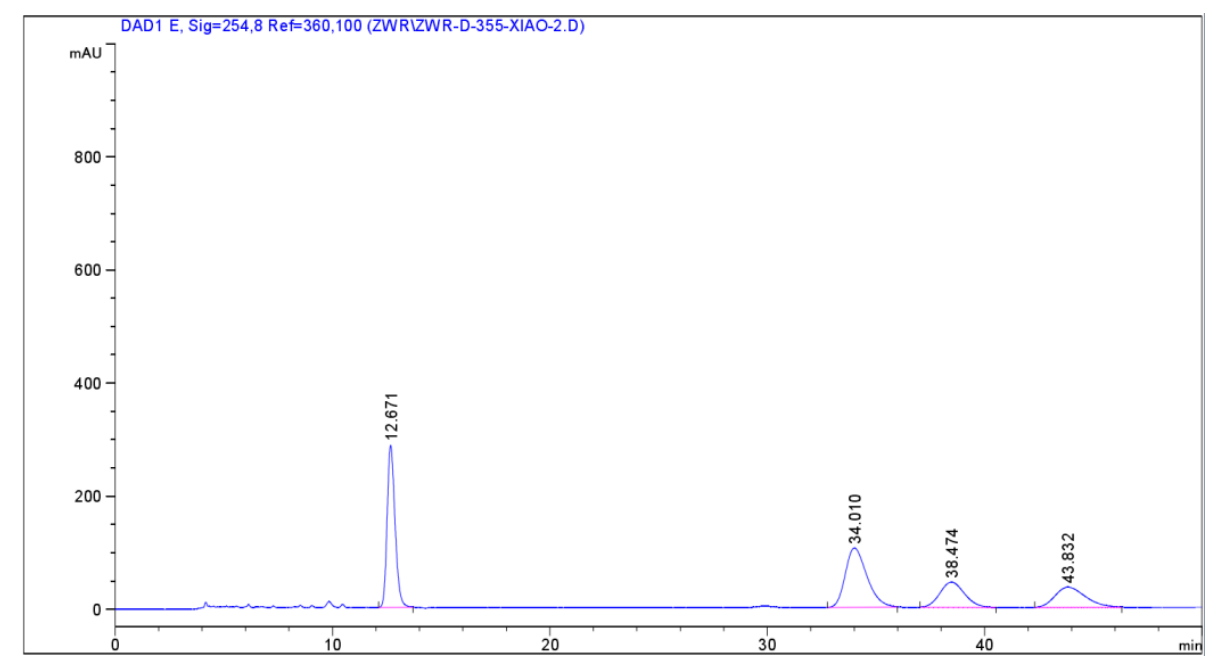

信号 1: DAD1 E， Sig=254,8 Ref=360,100

\begin{tabular}{|c|c|c|c|c|c|c|}
\hline 峰 & 留时间 类型 & 峰宽 & & 面积 & 峰面积 & \\
\hline$\#$ & [min $]$ & & {$[\mathrm{min}]$} & {$\left[\mathrm{mAU}{ }^{*} \mathrm{~S}\right]$} & [mAU ] & \% \\
\hline & & & & & & \\
\hline 1 & 12.671 & BB & 0.3912 & 7249.05469 & 286.51251 & 906 \\
\hline 2 & 34.010 & BB & 1.0588 & 7269.26611 & 105.11279 & 33.7846 \\
\hline 3 & 38.474 & 3B & 1.1984 & 3525.56128 & 44.59233 & 16.3854 \\
\hline 4 & 43.8321 & BB & 1.3683 & 3472.64795 & 35.59216 & 16.1394 \\
\hline
\end{tabular}

\section{HPLC spectrum of the chiral compound}

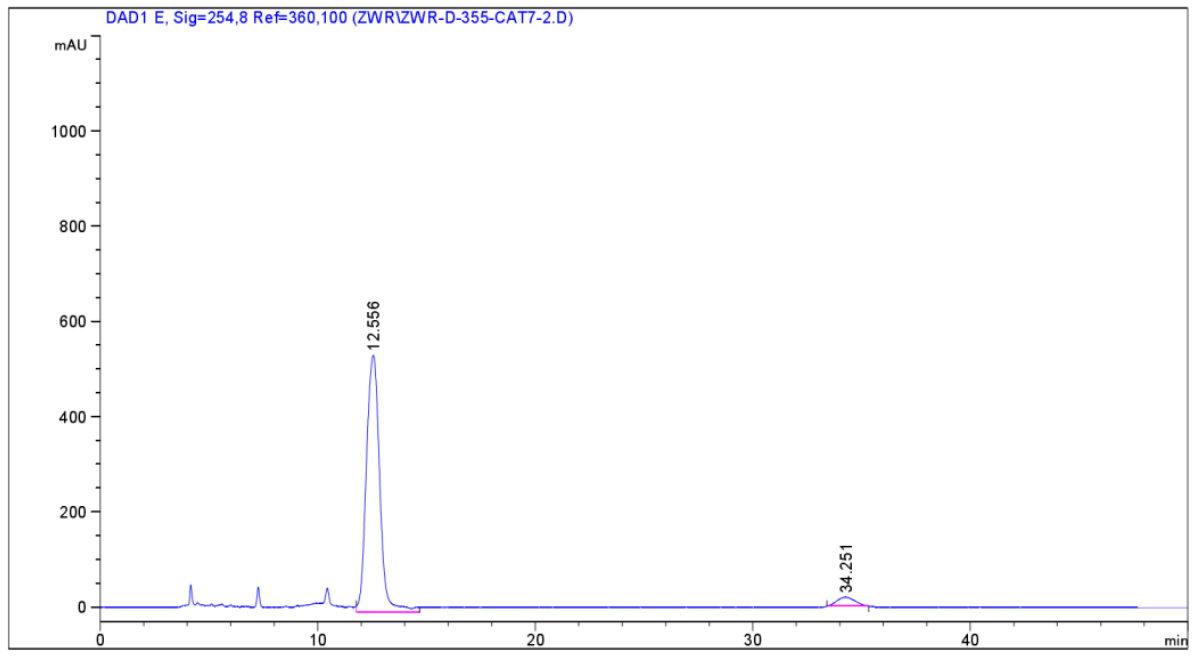

信号 1: DAD1 E， Sig=254, 8 Ref=360, 100

\begin{tabular}{|c|c|c|c|c|c|c|c|}
\hline & 留时间 类型 & 峰宽 & & 逢面积 & 峰高 & \multicolumn{2}{|c|}{ 峰面积 } \\
\hline \# & [min] & & [min ] & {$\left[\mathrm{mAU}{ }^{*} \mathrm{~S}\right]$} & & [mAU ] & $\%$ \\
\hline & & $\mathrm{R}$ & & 82.3 & & 53 & \\
\hline & & & 164 & A & & 17. & 3.98 \\
\hline
\end{tabular}




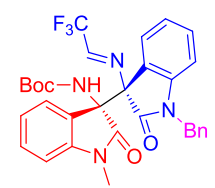

3j

HPLC spectrum of the racemate

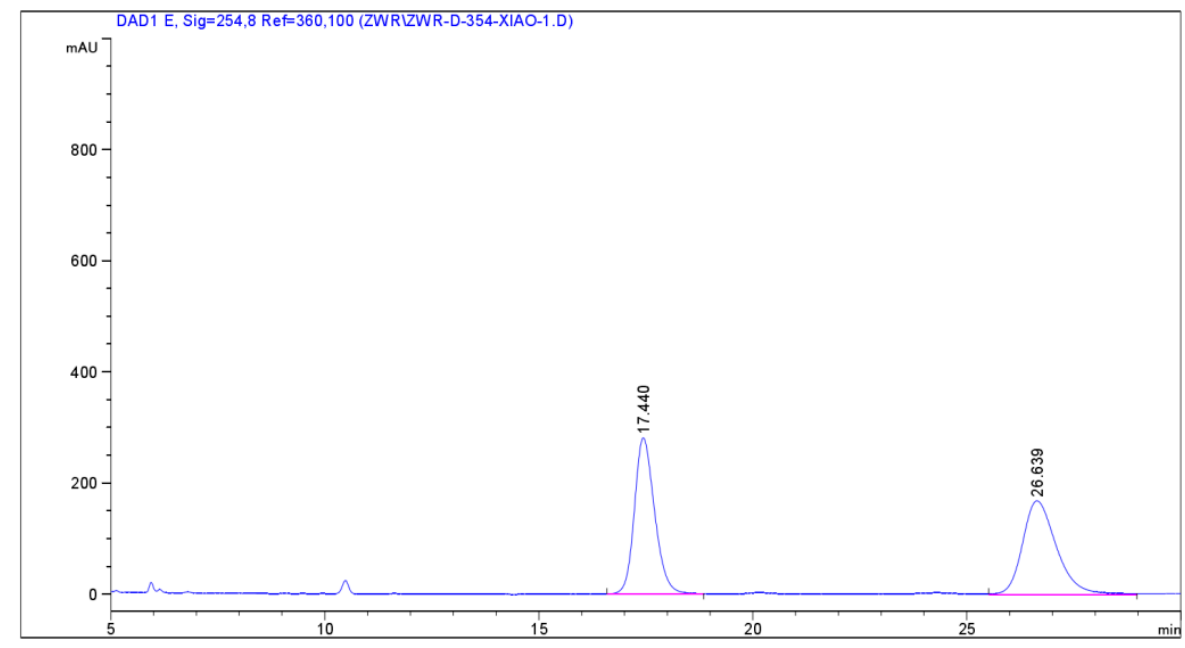

信号 1: DAD1 E， Sig=254,8 Ref=360,100

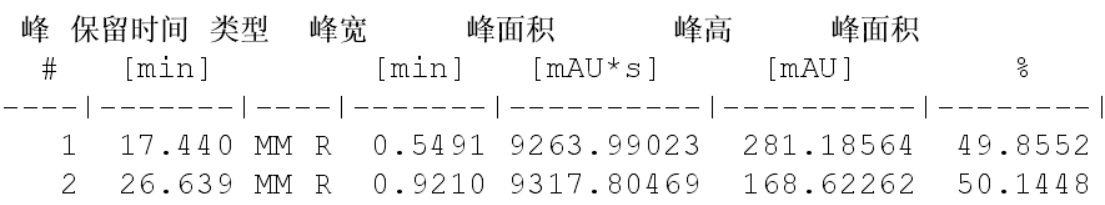

\section{HPLC spectrum of the chiral compound}

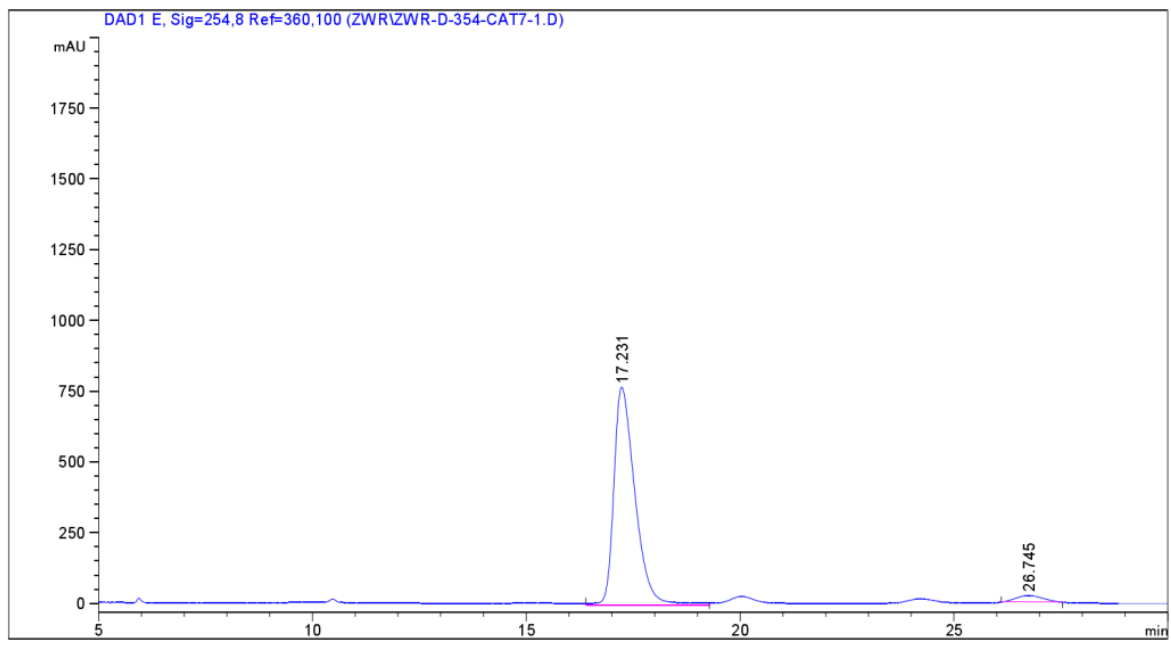

信号 1: DAD1 E, Sig=254,8 Ref=360,100

\begin{tabular}{ccccccc} 
峰 保留时间 类型 & 峰宽 & \multicolumn{3}{c}{ 峰面积 } & 峰高 & \multicolumn{2}{c}{ 峰面积 } \\
\# \\
{$[\mathrm{min}]$}
\end{tabular}




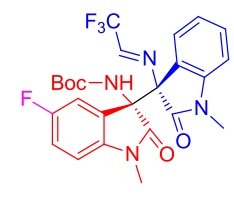

3k

HPLC spectrum of the racemate

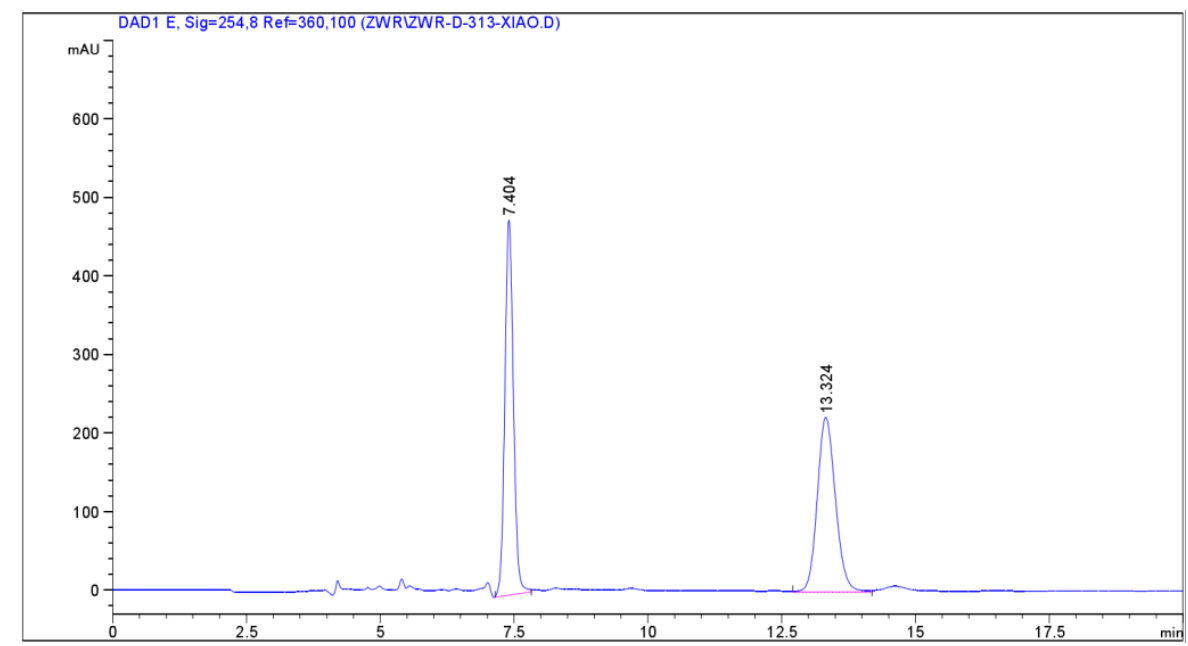

信号 1: DAD1 E， Sig=254,8 Ref=360,100

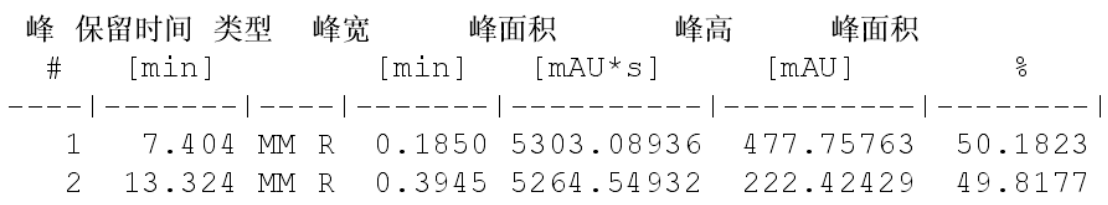

\section{HPLC spectrum of the chiral compound}

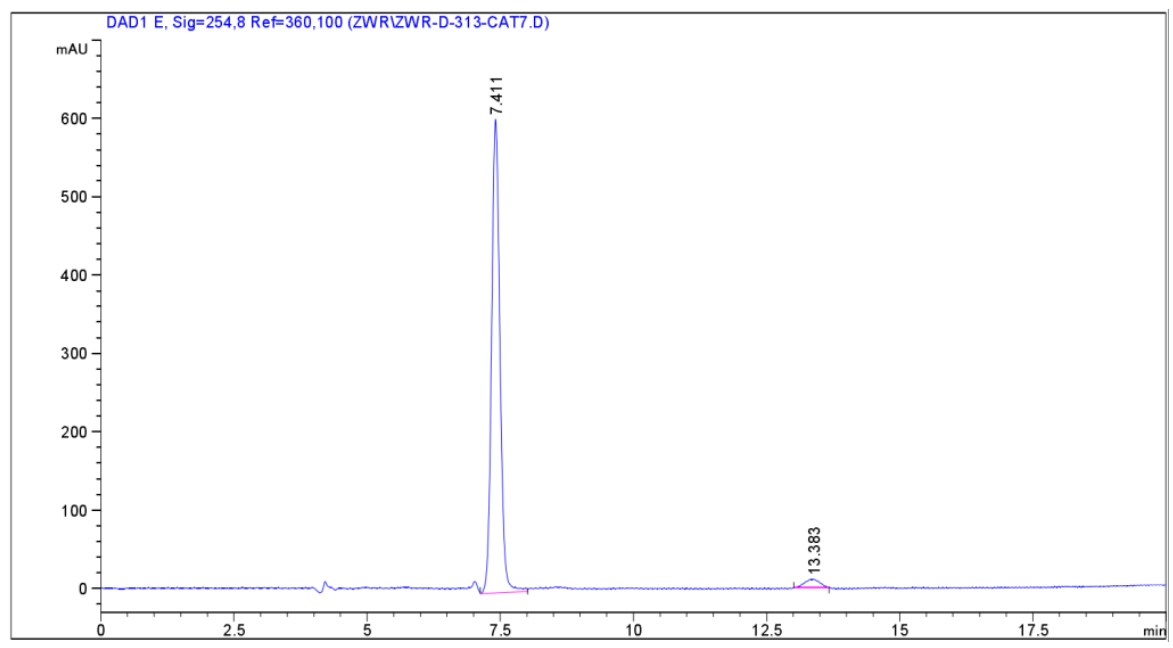

信号 1: DAD1 E， Sig=254,8 Ref $=360,100$

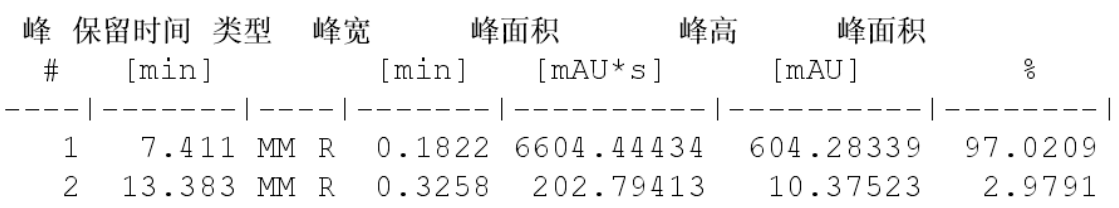




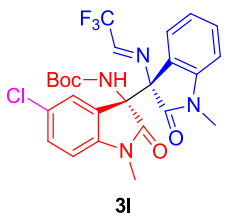

HPLC spectrum of the racemate

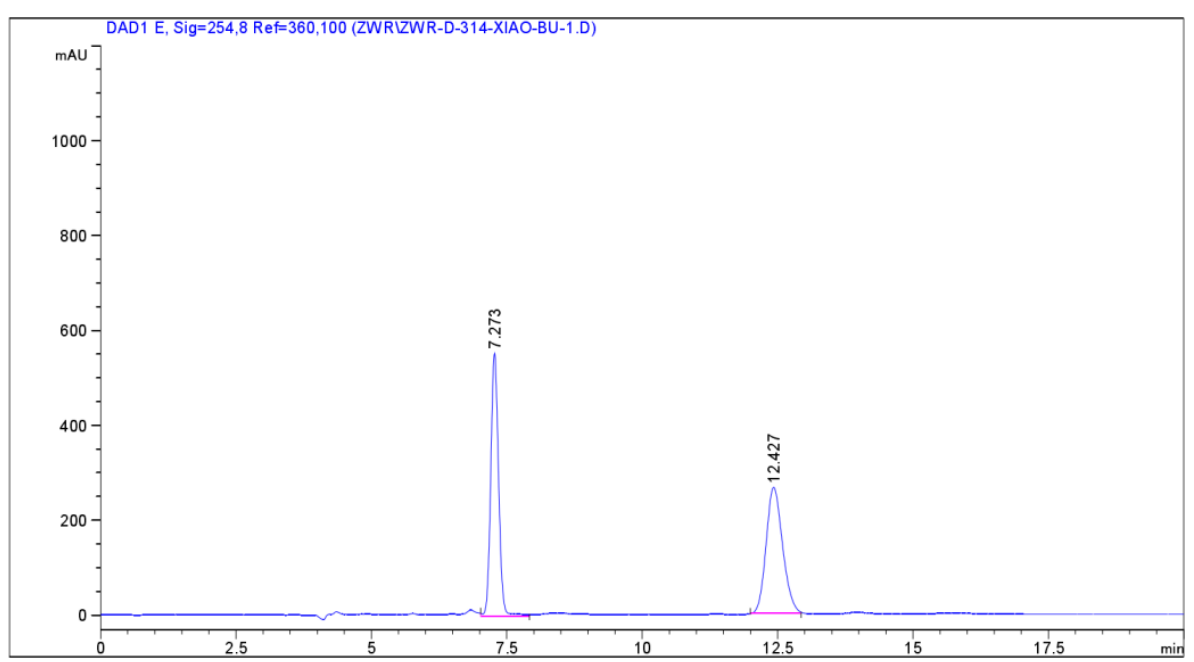

信号 1: DAD1 E， Sig=254,8 Ref=360,100

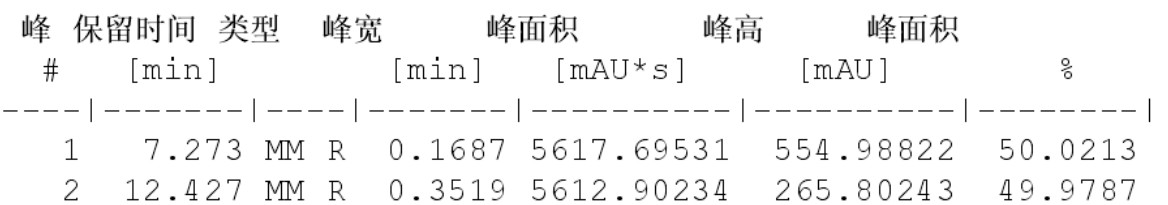

\section{HPLC spectrum of the chiral compound}

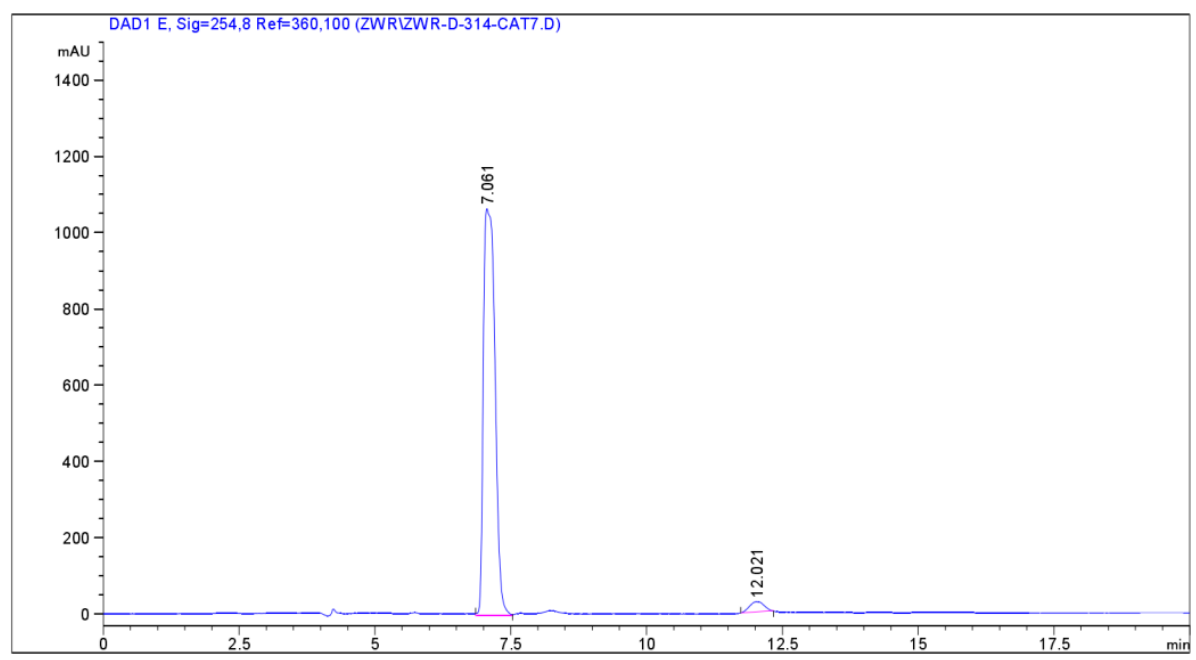


信号 1: DAD1 E, Sig=254,8 Ref=360,100

峰 保留时间 类型峰宽峰面积峰高峰面积

\# $[\mathrm{min}] \quad[\mathrm{min}] \quad\left[\mathrm{mAU}^{*} \mathrm{~S}\right] \quad[\mathrm{mAU}] \quad-$

$----|-------|----|-------|----------|----------|--------\mid$

$\begin{array}{llllllll}1 & 7.061 & \text { MM } & \text { R } & 0.2485 & 1.59149 e 4 & 1067.57349 & 97.0525\end{array}$

$\begin{array}{llllllll}2 & 12.021 & \text { MM R } & 0.3042 & 483.33224 & 26.48428 & 2.9475\end{array}$ 


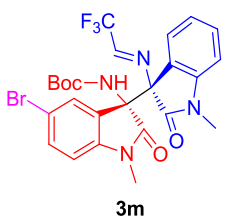

HPLC spectrum of the racemate

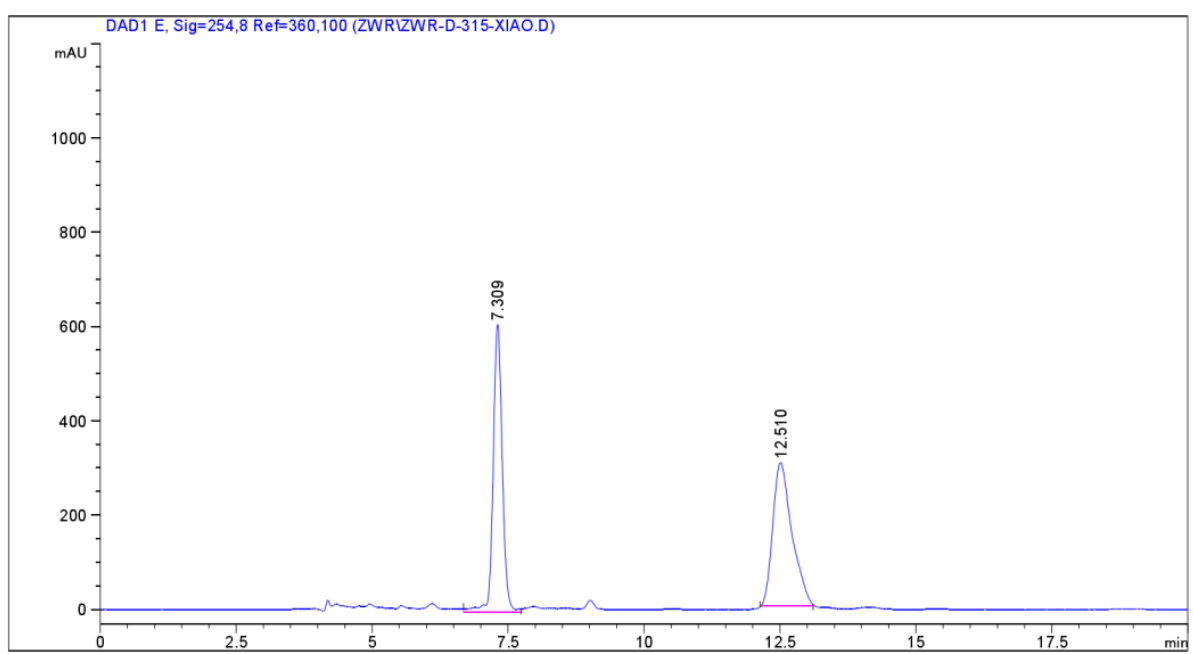

信号 1: DAD1 E， Sig=254,8 Ref=360,100

\begin{tabular}{|c|c|c|c|c|c|c|c|}
\hline 峰 & 留时间 类型 & 峰宽 & & 面积 & 峰高 & \multicolumn{2}{|l|}{ 峰面积 } \\
\hline \# & {$[\mathrm{min}]$} & & [min ] & {$\left[\mathrm{mAU}{ }^{*} \mathrm{~S}\right]$} & & [mAU ] & \% \\
\hline 1 & $7.309 \mathrm{MI}$ & $\mathrm{R}$ & 0.1960 & 7172.796 & & 609.92456 & 49.2046 \\
\hline 2 & $12.510 \mathrm{MI}$ & $\mathrm{R}$ & 0.4065 & 7404.706 & & 303.63327 & 50.7954 \\
\hline
\end{tabular}

\section{HPLC spectrum of the chiral compound}

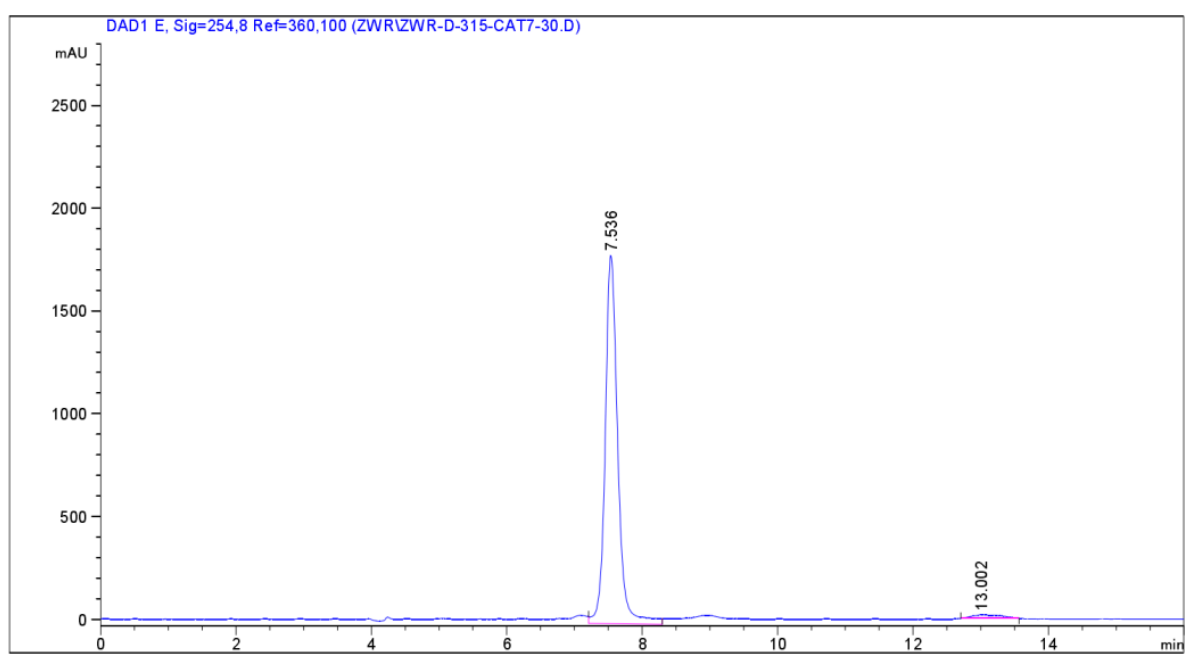

信号 1: DAD1 E， Sig=254,8 Ref=360,100

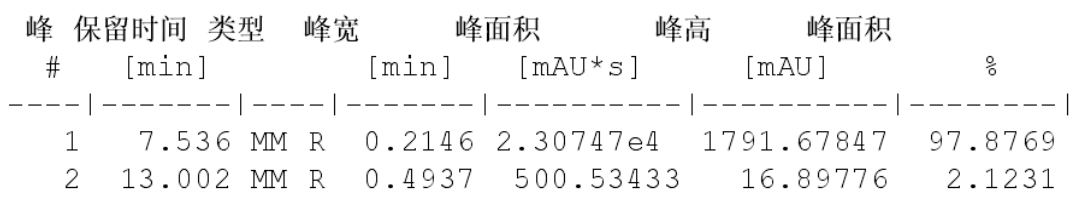




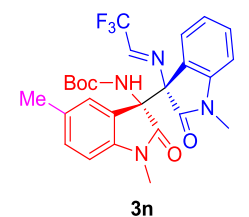

HPLC spectrum of the racemate

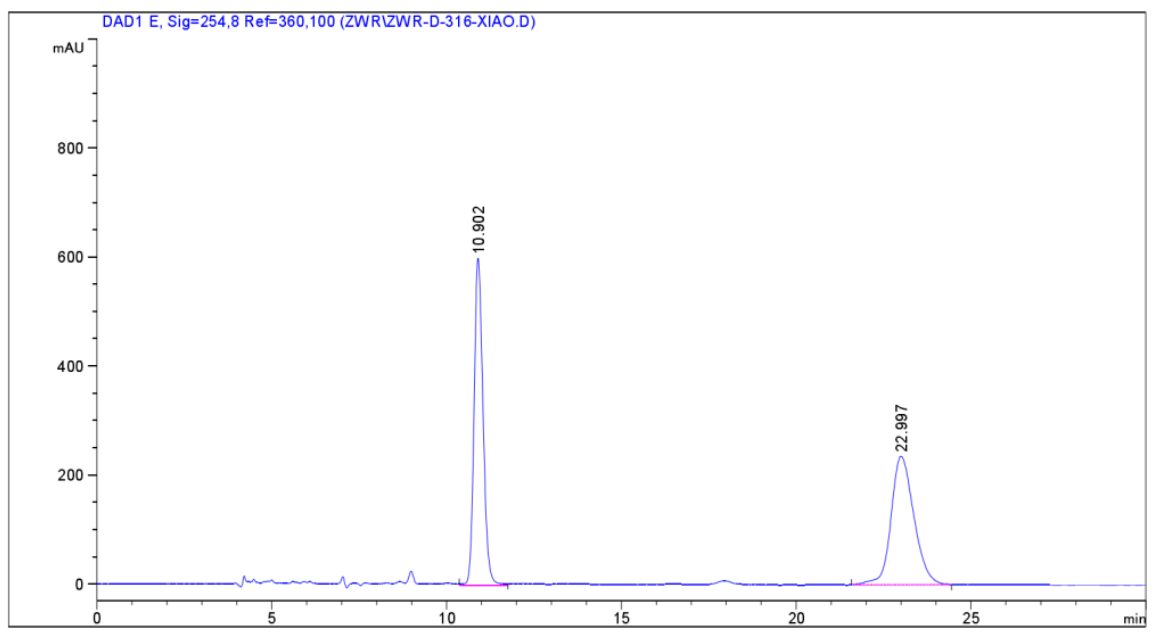

信号 1: DAD1 E， Sig=254,8 Ref=360,100

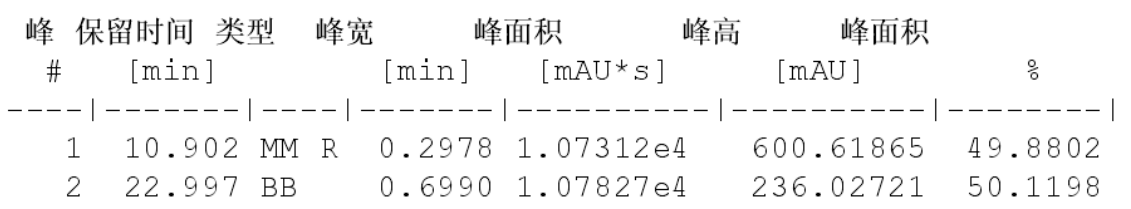

\section{HPLC spectrum of the chiral compound}

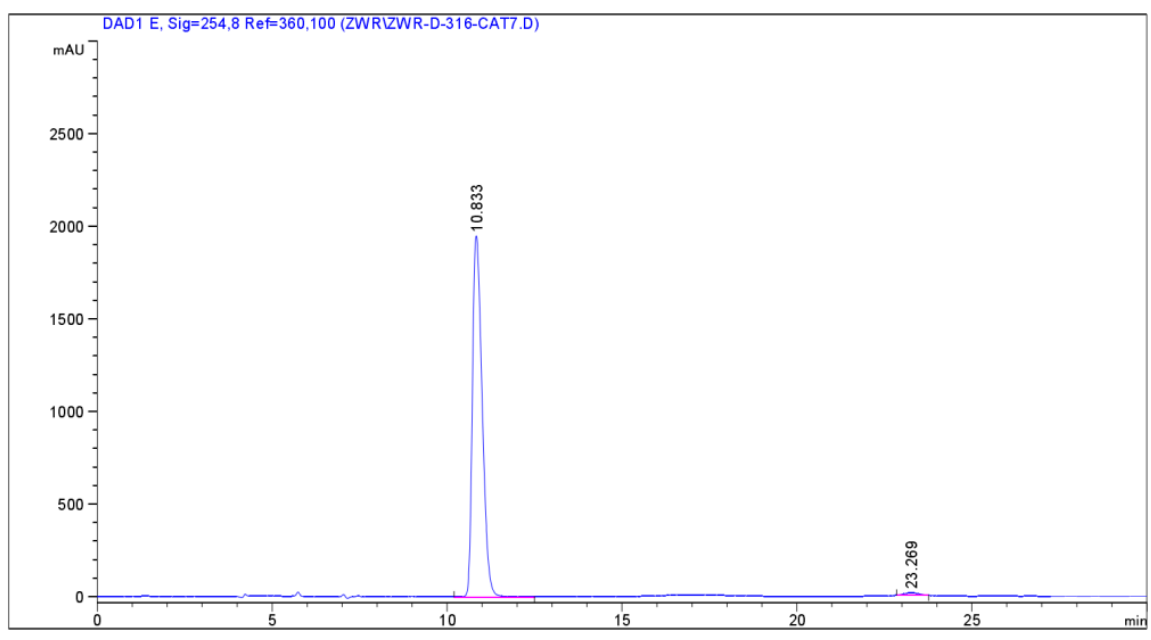

信号 1: DAD1 E， Sig=254,8 Ref=360,100

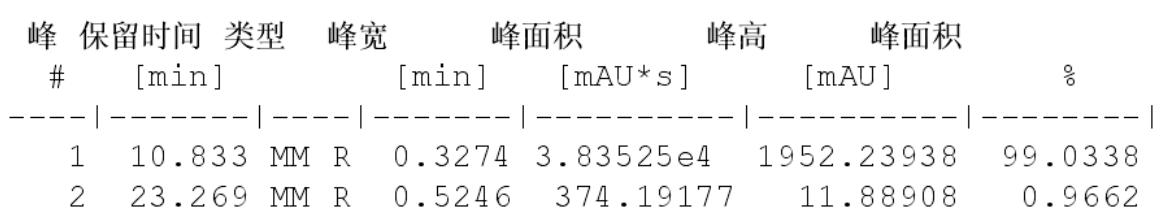




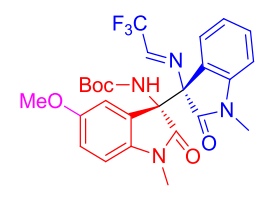

30

HPLC spectrum of the racemate

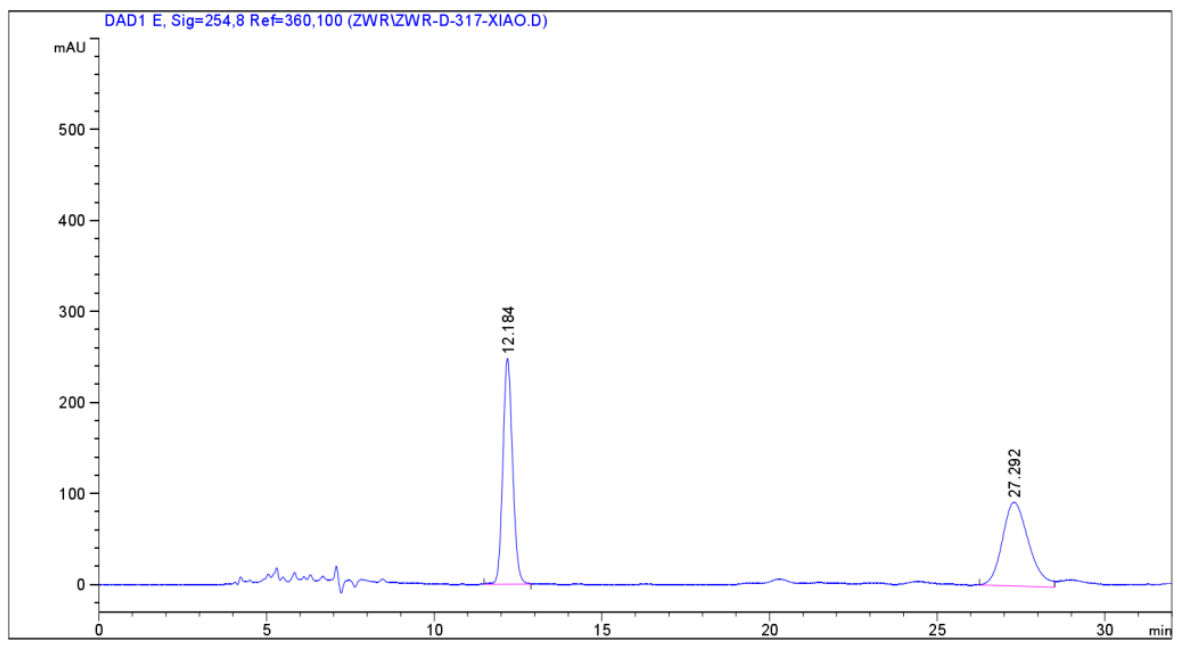

信号 1: DAD1 E， Sig=254,8 Ref=360, 100

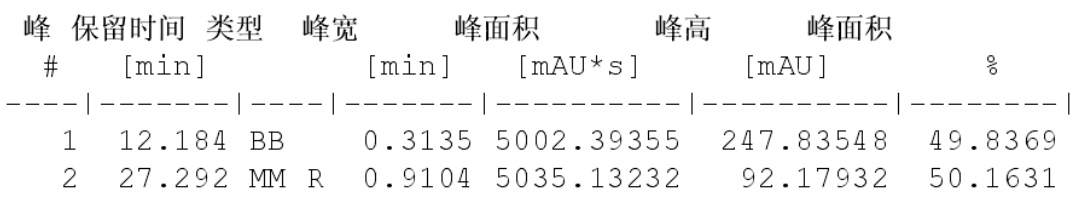

\section{HPLC spectrum of the chiral compound}

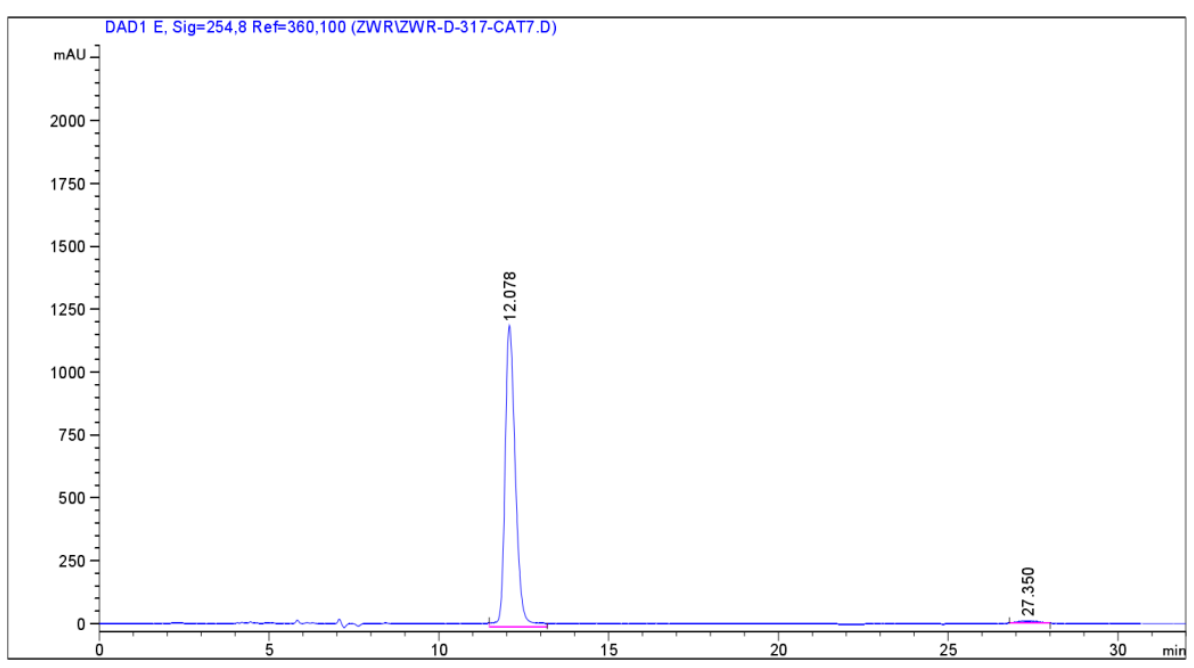

信号 1: DAD1 E, Sig=254,8 Ref=360,100

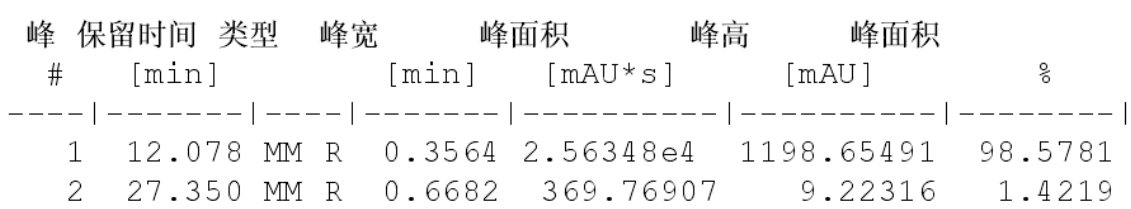




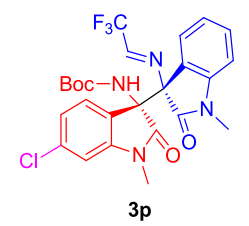

HPLC spectrum of the racemate

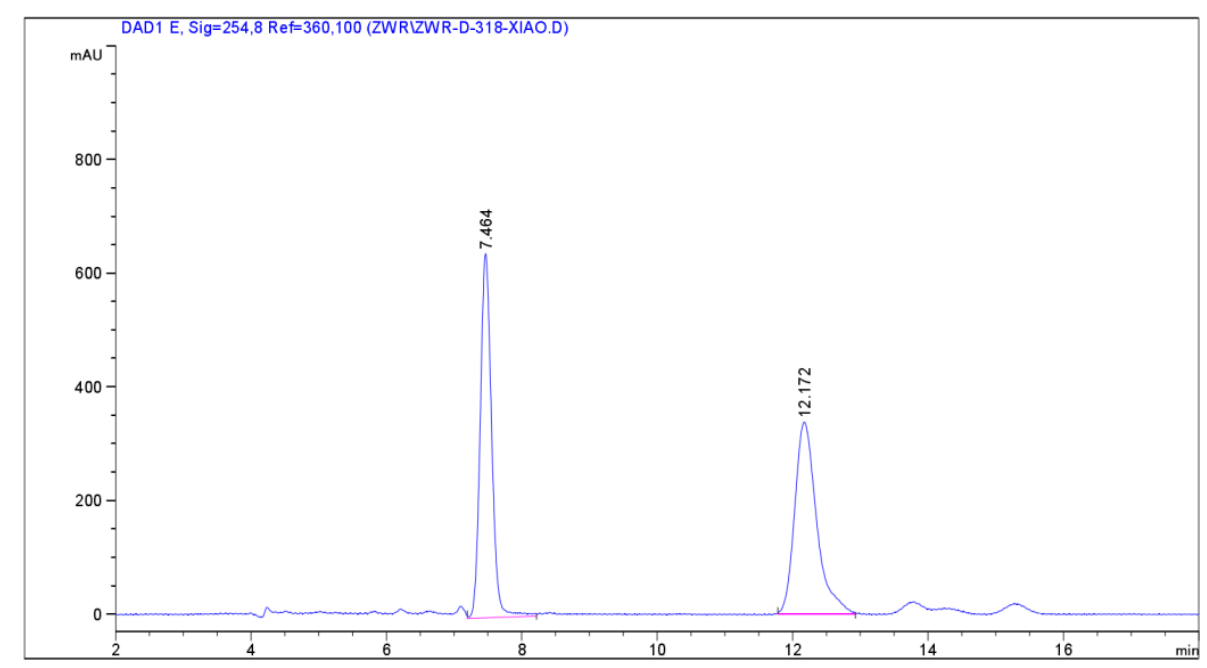

信号 1: DAD1 E， Sig=254,8 $\operatorname{Ref}=360,100$

\begin{tabular}{|c|c|c|c|c|c|c|c|}
\hline \multirow{2}{*}{$\begin{array}{c}\text { 峰 } \\
\text { \# }\end{array}$} & 留时间 类型 & 峰宽 & & 面积 & 峰高 & \multicolumn{2}{|l|}{ 峰面积 } \\
\hline & [min $]$ & & {$[\mathrm{min}]$} & {$\left[\mathrm{mAU}^{*} \mathrm{~s}\right]$} & & [mAU ] & $\%$ \\
\hline & & & & & & & \\
\hline 1 & 7.464 & $\mathrm{R}$ & 0.1948 & 7488.815 & 43 & 640.75610 & 498 \\
\hline 2 & $12.172 \mathrm{M}$ & $\mathrm{R}$ & 0.3726 & 7533.952 & 64 & 337.01459 & 50.1502 \\
\hline
\end{tabular}

\section{HPLC spectrum of the chiral compound}

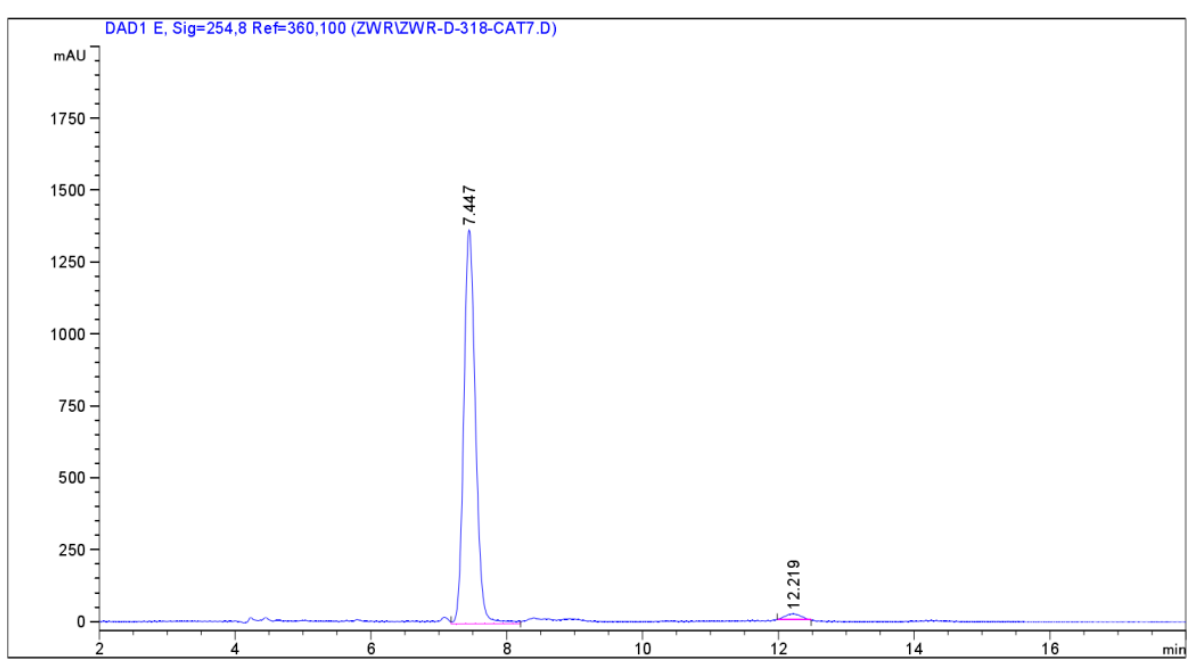

信号 1: DAD1 E, Sig=254,8 Ref=360,100

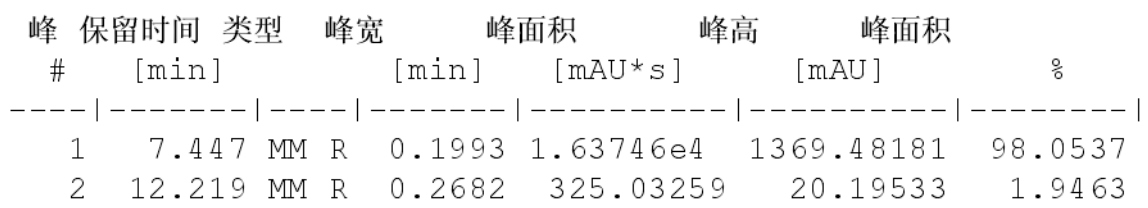




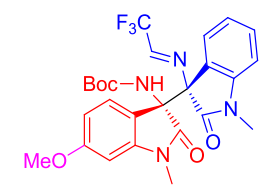

$3 q$

HPLC spectrum of the racemate

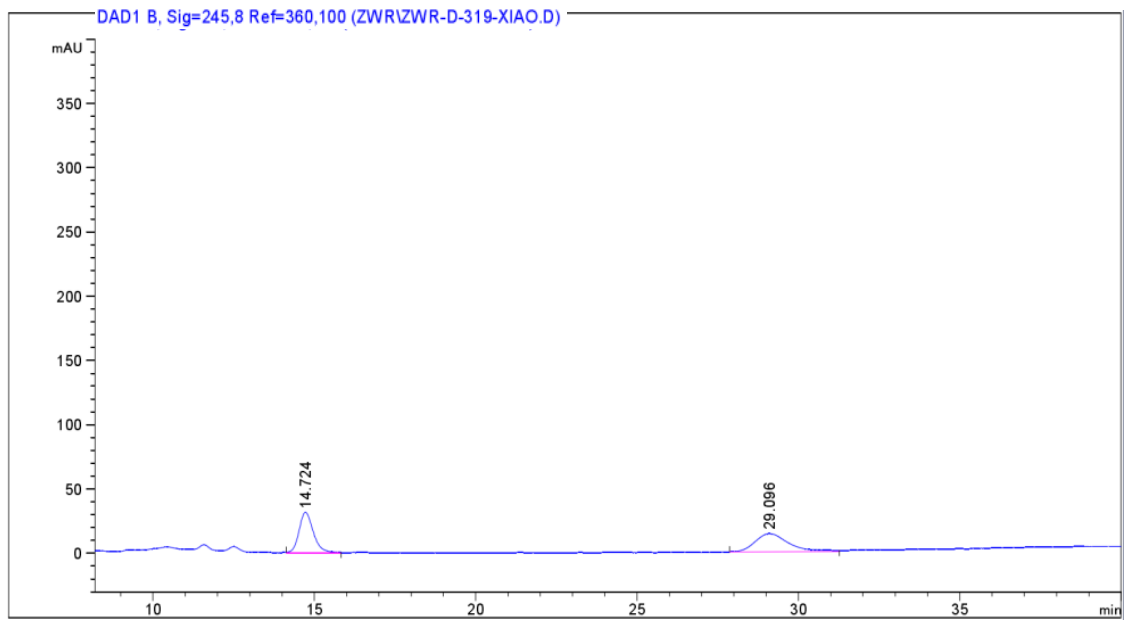

信号 1: DAD1 B， Sig=245,8 Ref $=360,100$

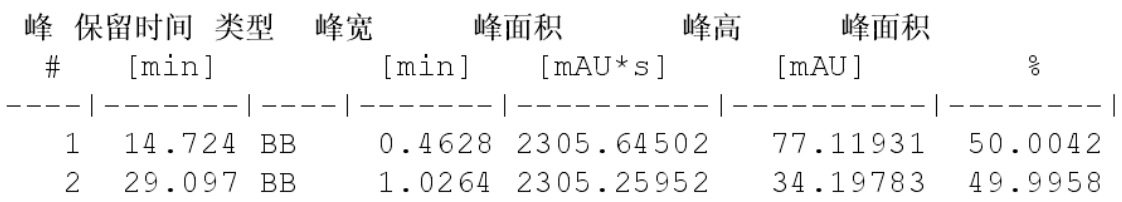

\section{HPLC spectrum of the chiral compound}

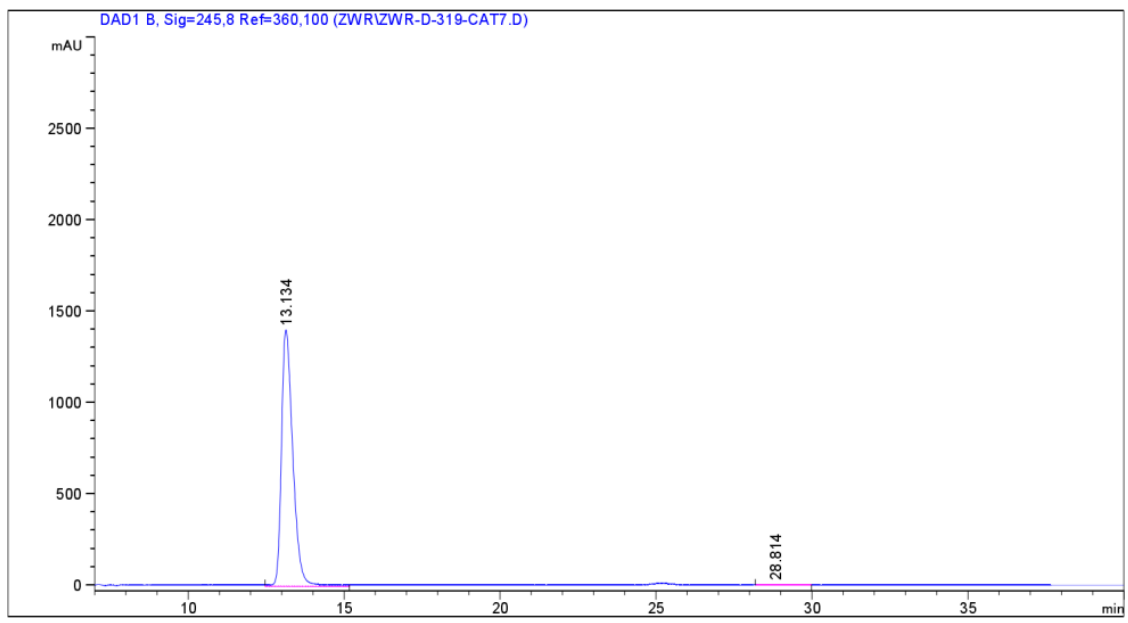

信号 1: DAD1 B， Sig=245,8 Ref $=360,100$

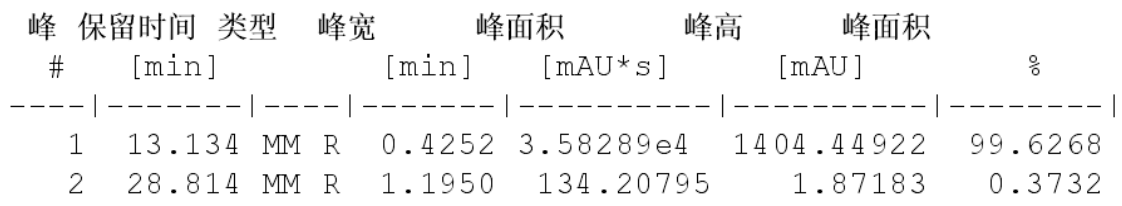




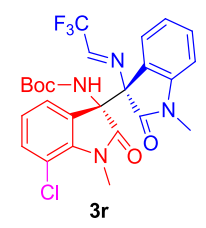

HPLC spectrum of the racemate

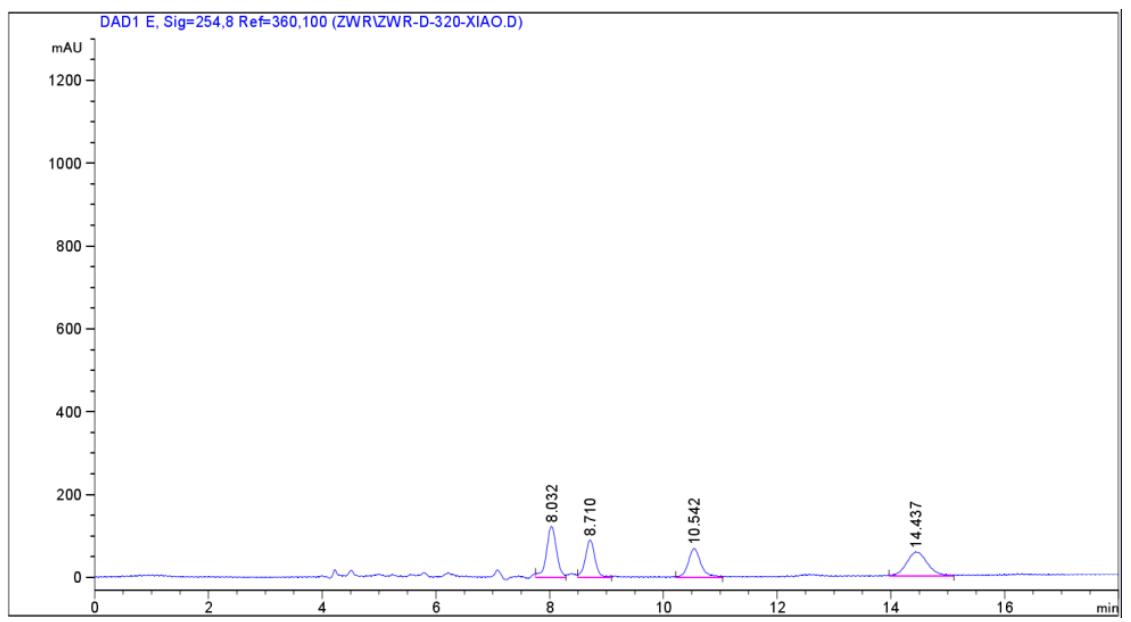

信号 1: DAD1 E， Sig=254,8 Ref=360,100

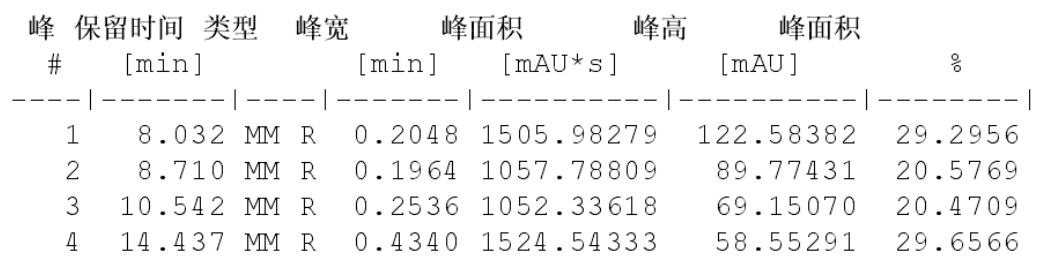

\section{HPLC spectrum of the chiral compound}

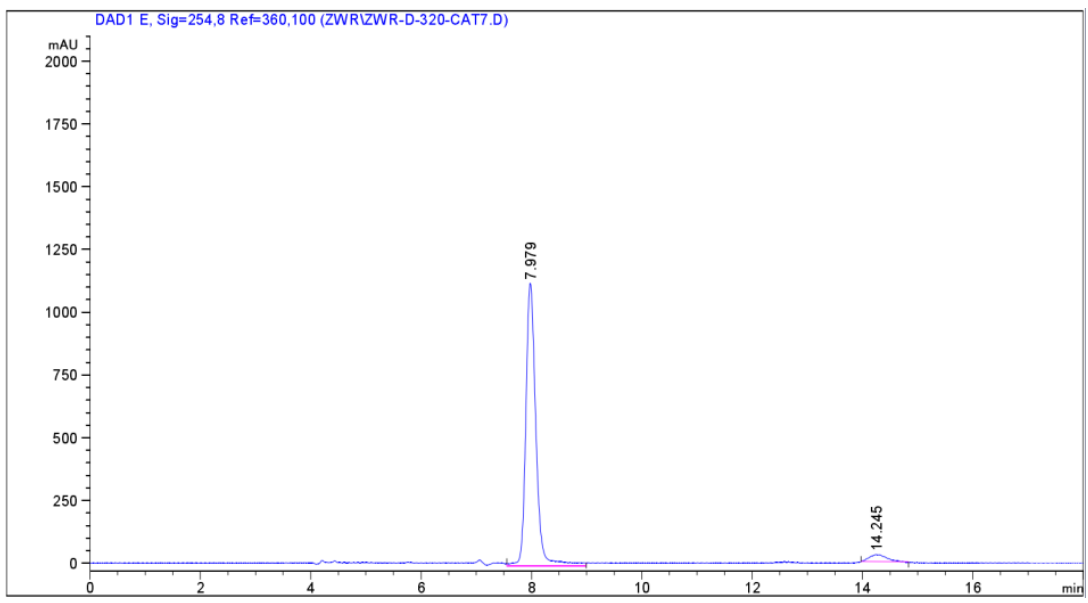

信号 1: DAD1 E， Sig=254,8 Ref=360, 100

\begin{tabular}{|c|c|c|c|c|c|c|c|}
\hline 峰 & 保留时间 类型 & 峰宽 & & 面积 & 峰高 & \multicolumn{2}{|l|}{ 峰面积 } \\
\hline$\#$ & [min ] & & [min ] & {$\left[\mathrm{mAU}{ }^{*} \mathrm{~s}\right]$} & & {$[\mathrm{mAU}]$} & $\%$ \\
\hline 1 & $979 \mathrm{MM}$ & $\mathrm{R}$ & 0.2132 & $1.44302 \mathrm{e}$ & & 1128.30896 & 96.0785 \\
\hline 2 & $14.245 \mathrm{MM}$ & $\mathrm{R}$ & 0.3593 & 588.983 & 83 & 27.32102 & 3.9215 \\
\hline
\end{tabular}




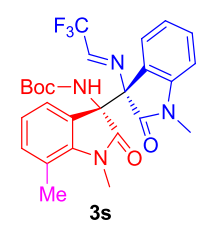

HPLC spectrum of the racemate

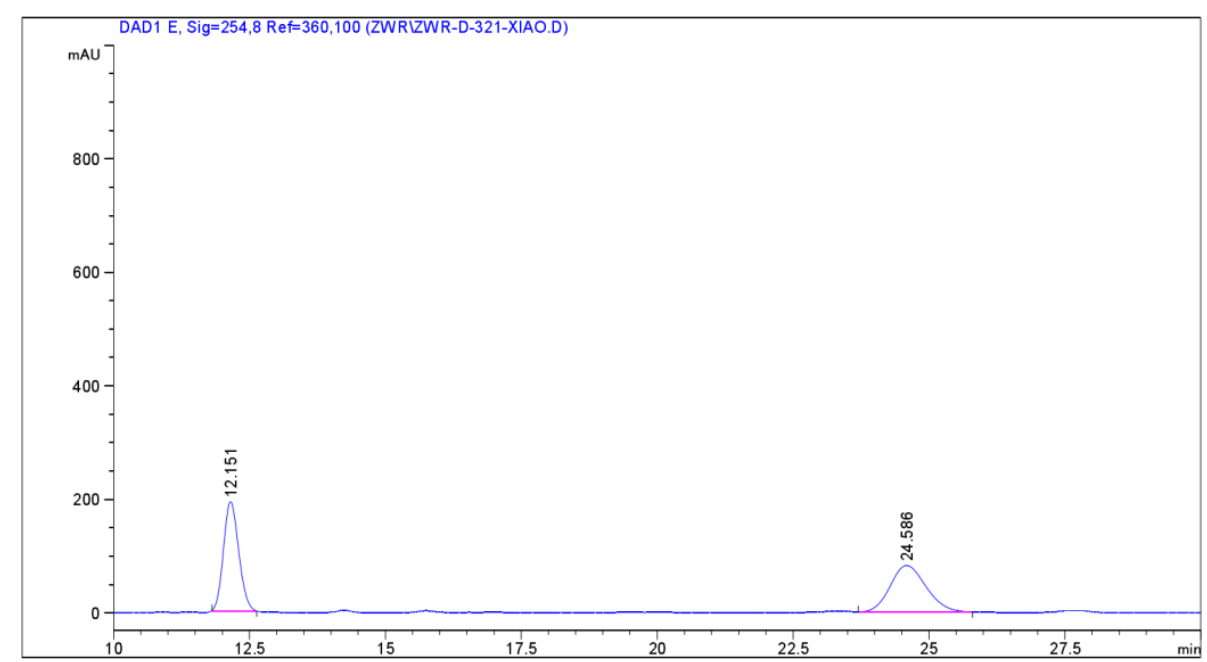

信号 1: DAD1 E， Sig=254,8 Ref=360,100

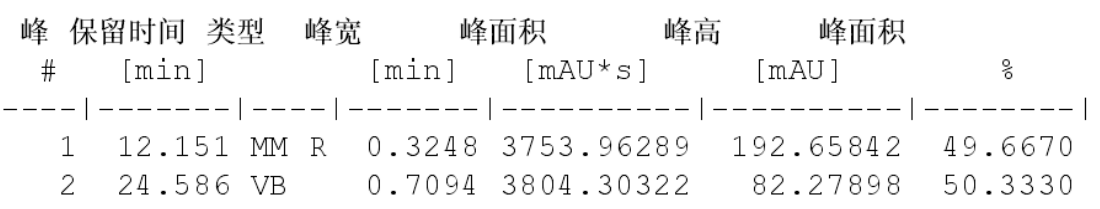

\section{HPLC spectrum of the chiral compound}

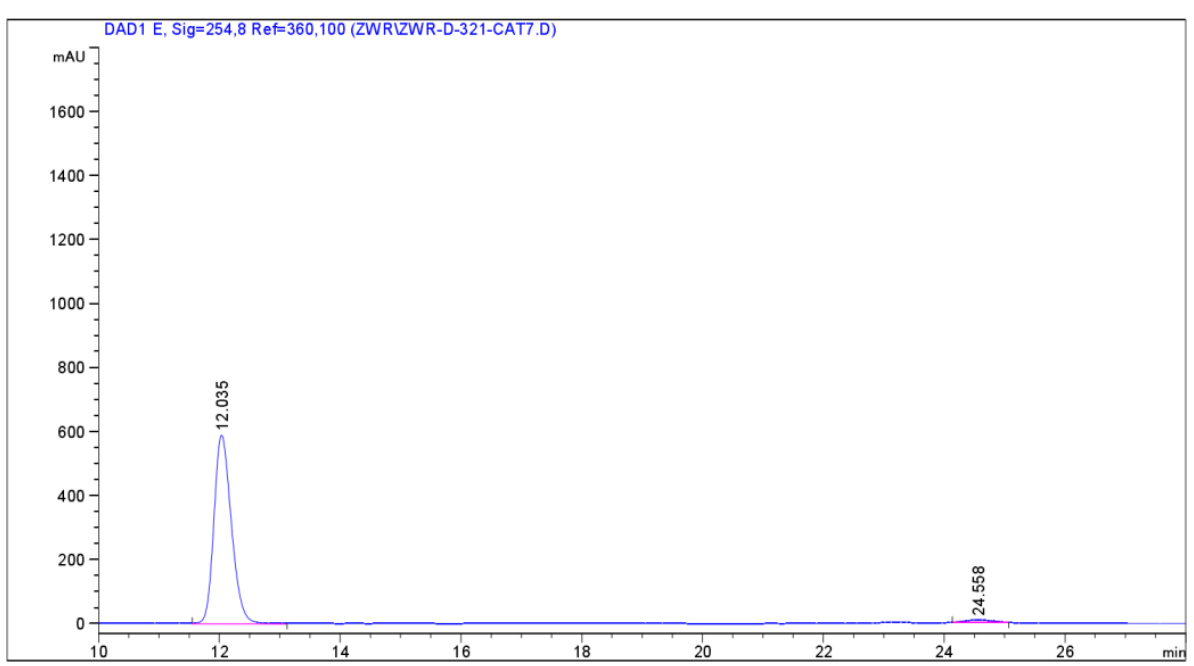

信号 1: DAD1 E, Sig=254,8 Ref=360,100

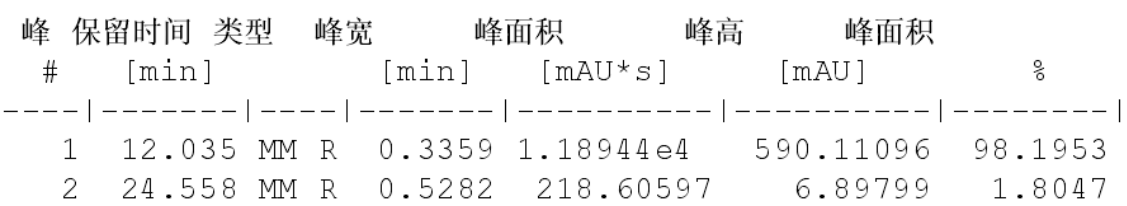




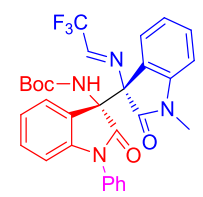

3t

HPLC spectrum of the racemate

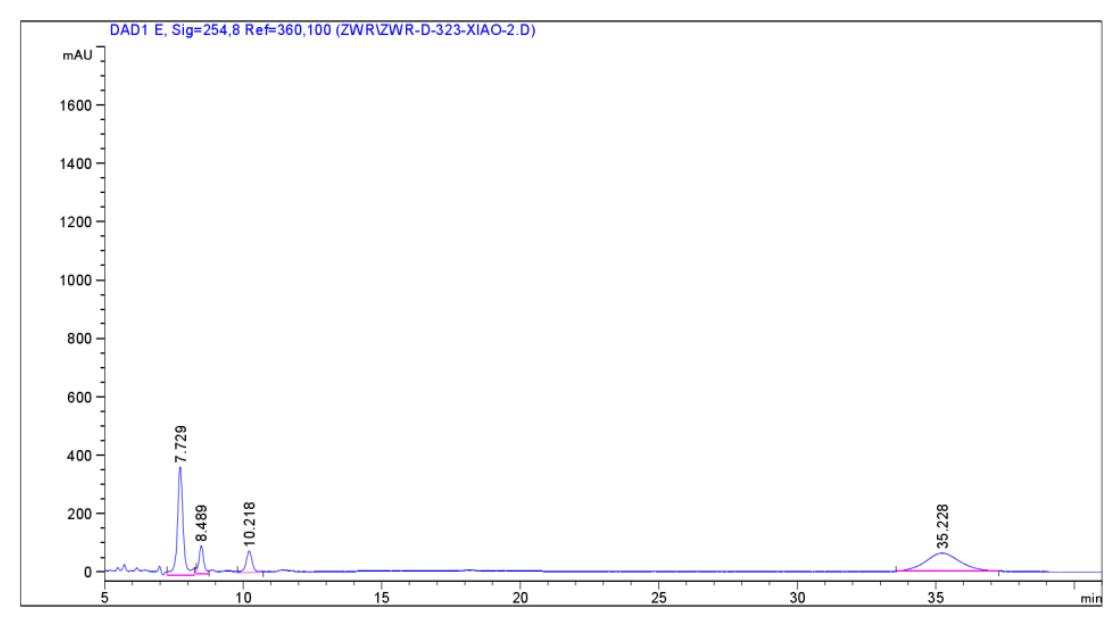

信号 1: DAD1 E， Sig=254, 8 Ref=360,100

\begin{tabular}{|c|c|c|c|c|c|c|c|}
\hline 峰 住 & 留时间 类 & & 峰宽 & & 面积 & 峰面积 & \\
\hline \# & [min $]$ & & & [min ] & {$[\mathrm{mAU} * \mathrm{~s}]$} & [mAU ] & $\%$ \\
\hline & & & & & - & -------- & ------- \\
\hline 1 & 7.729 & $\mathrm{MM}$ & $\mathrm{R}$ & 0.2414 & 5374.12744 & 371.01923 & 41.1859 \\
\hline 2 & 8.489 & MM & $\mathrm{R}$ & 0.1991 & 1152.49268 & 96.46130 & 8.8324 \\
\hline 3 & 10.218 & VB & & 0.2402 & 1158.86597 & 73.38143 & 8.8812 \\
\hline 4 & 35.228 & MM & $\mathrm{R}$ & 1.4462 & 5362.97900 & 61.80388 & 41.1005 \\
\hline
\end{tabular}

\section{HPLC spectrum of the chiral compound}

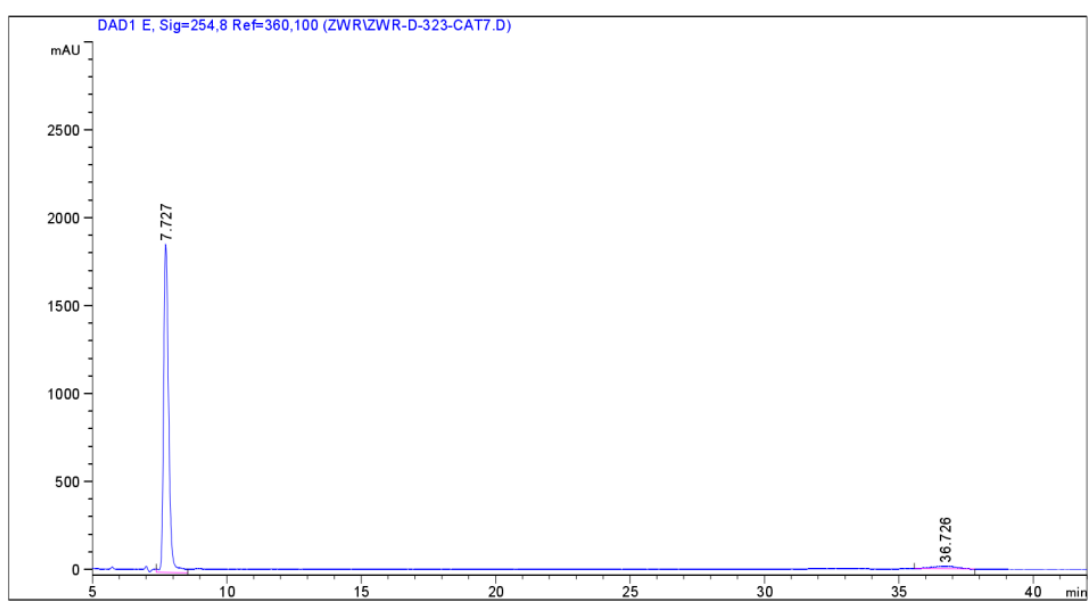

信号 1: DAD1 E, Sig=254,8 $\operatorname{Ref}=360,100$

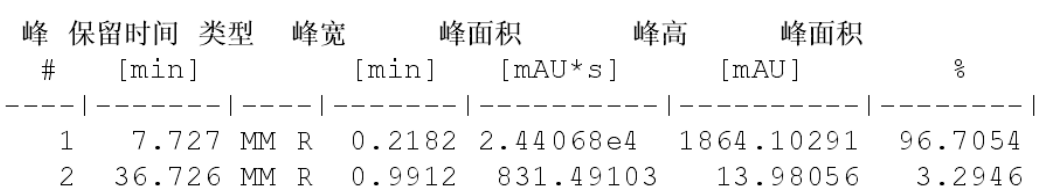




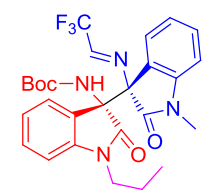

$3 \mathbf{u}$

HPLC spectrum of the racemate

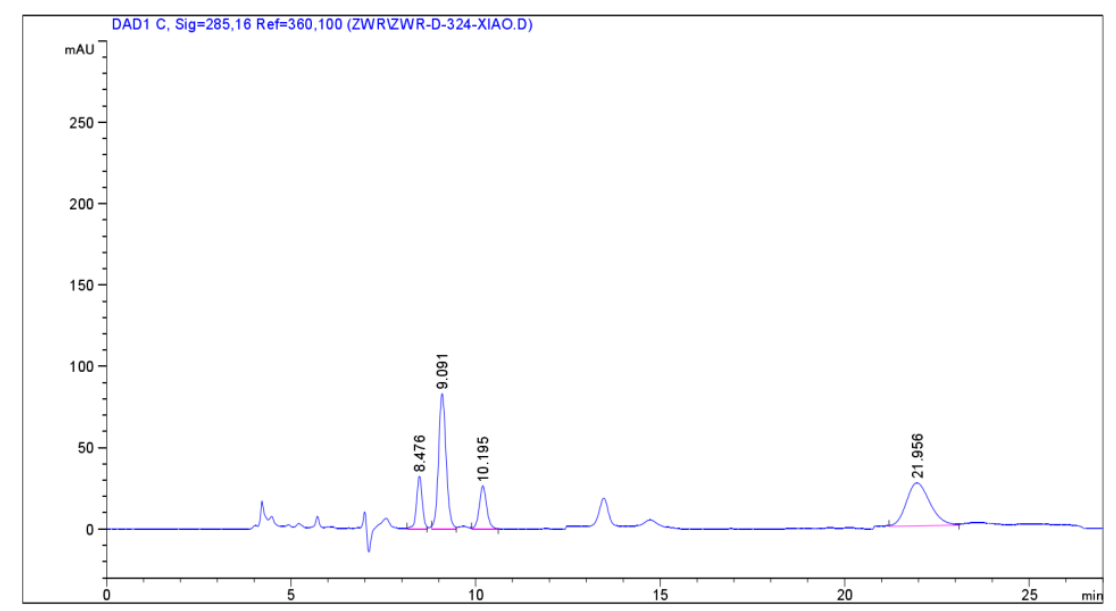

信号 1: DAD1 C, Sig=285,16 Ref=360,100

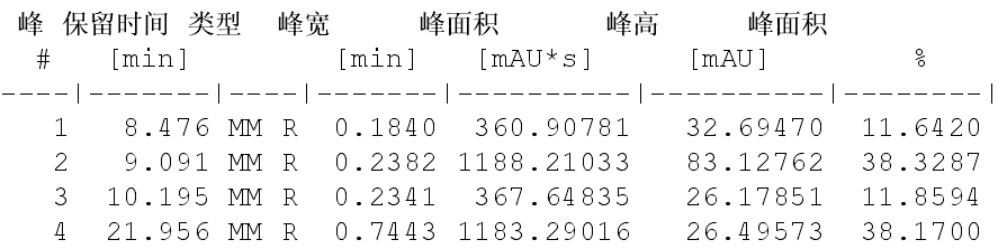

\section{HPLC spectrum of the chiral compound}

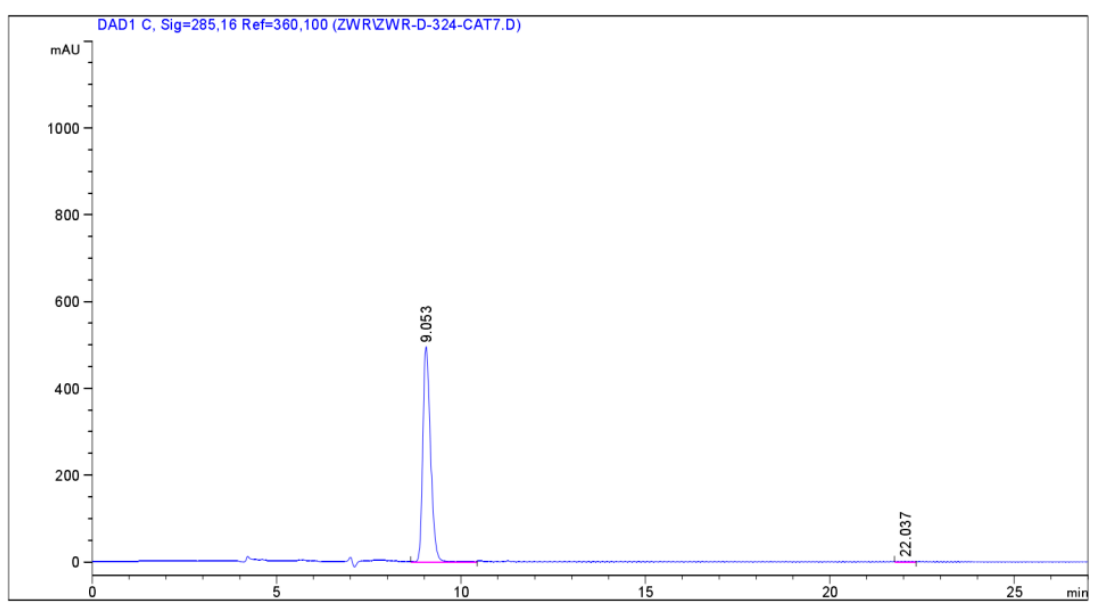

信号 1: DAD1 C, Sig=285,16 Ref=360,100

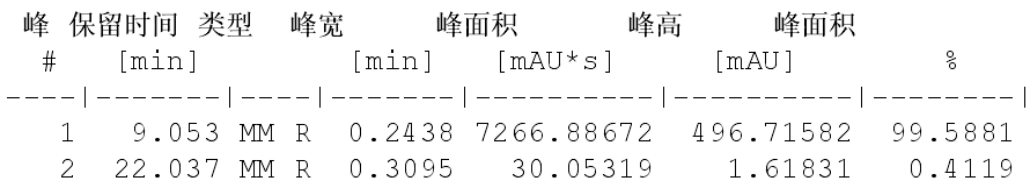




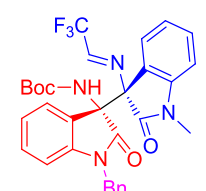

$3 v$

HPLC spectrum of the racemate

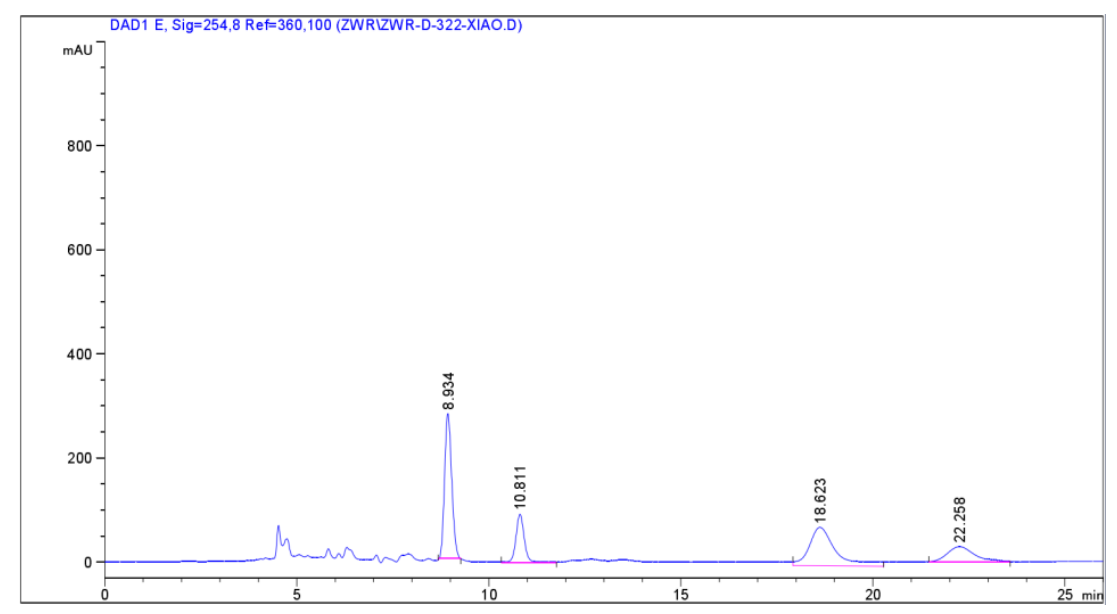

信号 1: DAD1 E， Sig=254,8 $\operatorname{Ref}=360,100$

\begin{tabular}{|c|c|c|c|c|c|c|c|}
\hline 峰 1 & 留时间 类 & & 峰宽 & & 面积 & 峰面积 & \\
\hline \# & [min] & & & [min $]$ & {$\left[\mathrm{mAU}{ }^{*} \mathrm{~s}\right]$} & [mAU ] & $\%$ \\
\hline & & & & ----- & ---------- & --------- & ------- \\
\hline 1 & 8.934 & MM & $\mathrm{R}$ & 0.2150 & 3595.37915 & 278.65433 & 35.6724 \\
\hline 2 & 10.811 & MM & $\mathrm{R}$ & 0.2582 & 1439.80823 & 92.95214 & 14.2854 \\
\hline 3 & 18.623 & MM & $\mathrm{R}$ & 0.8122 & 3608.78638 & 74.05626 & 35.8054 \\
\hline 4 & 22.258 & MM & $\mathrm{R}$ & 0.8218 & 1434.90442 & 29.10219 & 14.2367 \\
\hline
\end{tabular}

\section{HPLC spectrum of the chiral compound}

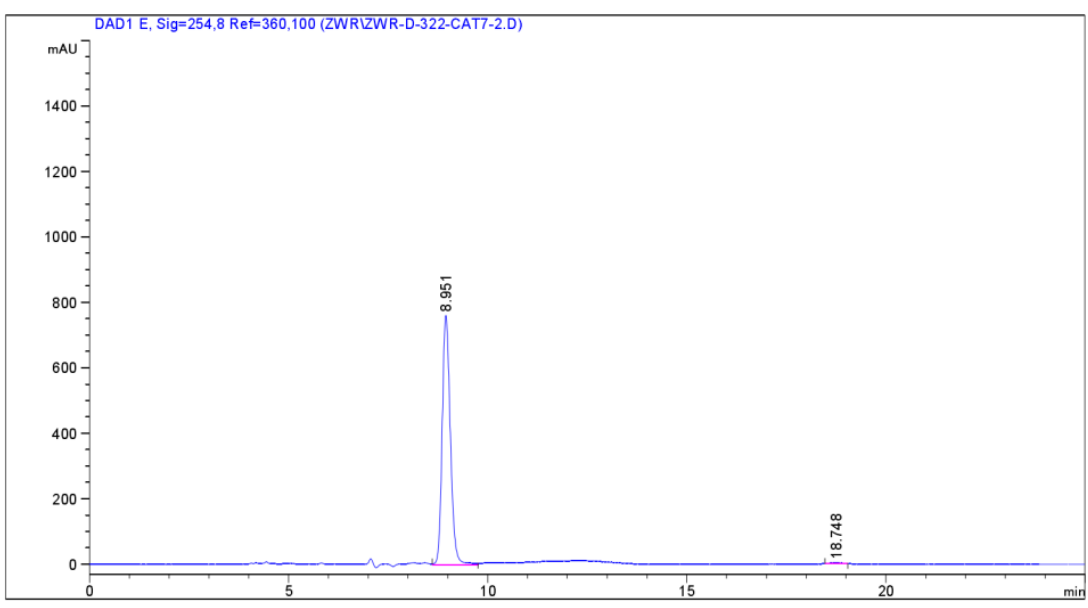

信号 1: DAD1 E, Sig=254, 8 Ref $=360,100$

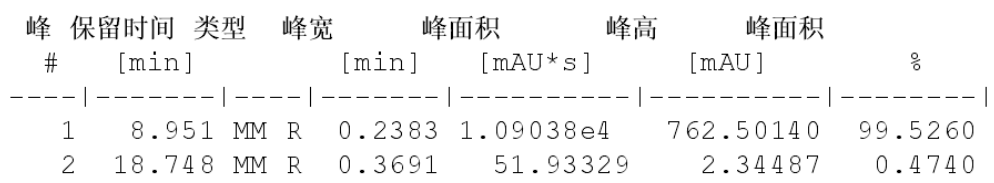




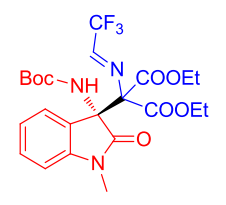

$8 a$

\section{HPLC spectrum of the racemate}

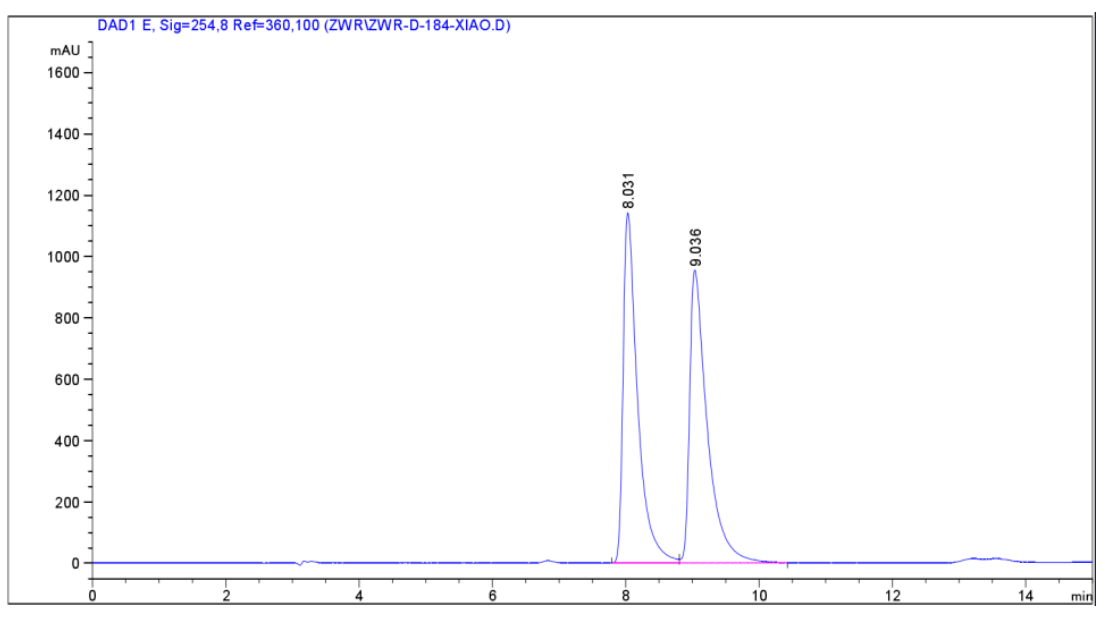

信号 1: DAD1 E, Sig=254,8 Ref=360,100

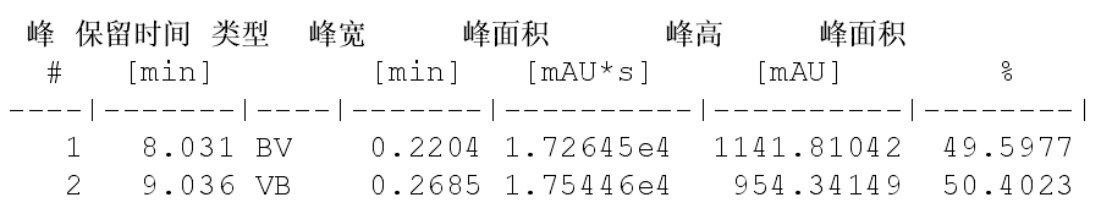

\section{HPLC spectrum of the chiral compound}

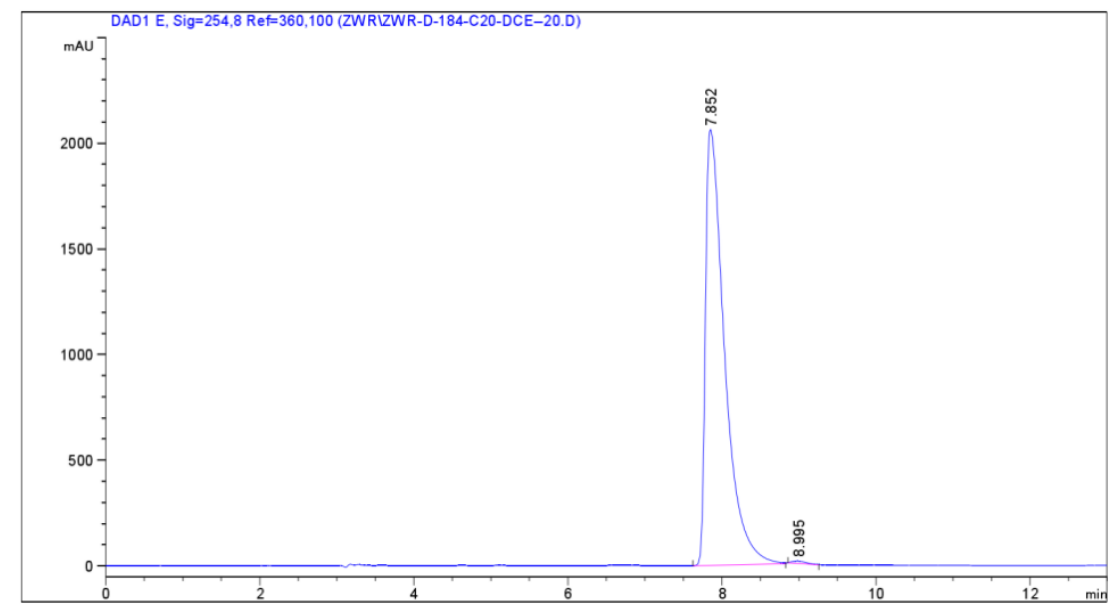

信号 1: DAD1 E, Sig=254,8 Ref=360,100

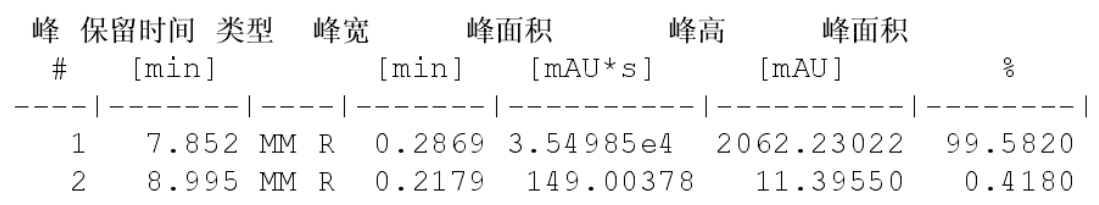




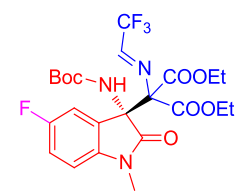

$8 b$

\section{HPLC spectrum of the racemate}

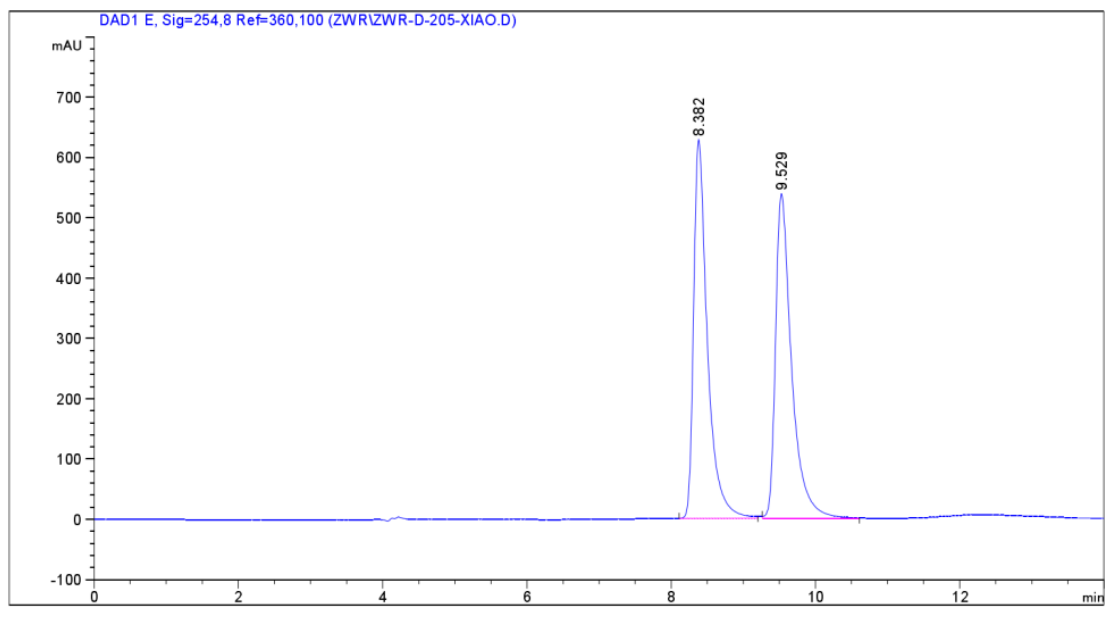

信号 1: DAD1 E， Sig=254, 8 Ref $=360,100$

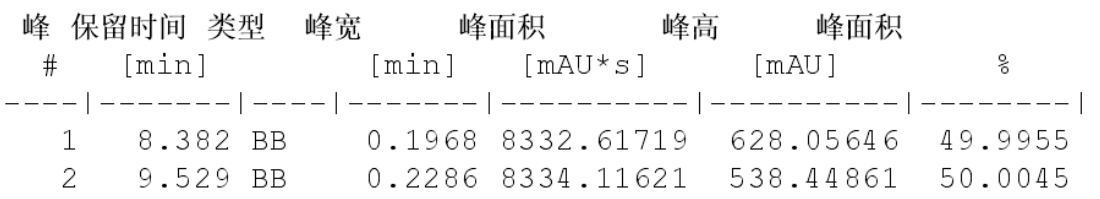

\section{HPLC spectrum of the chiral compound}

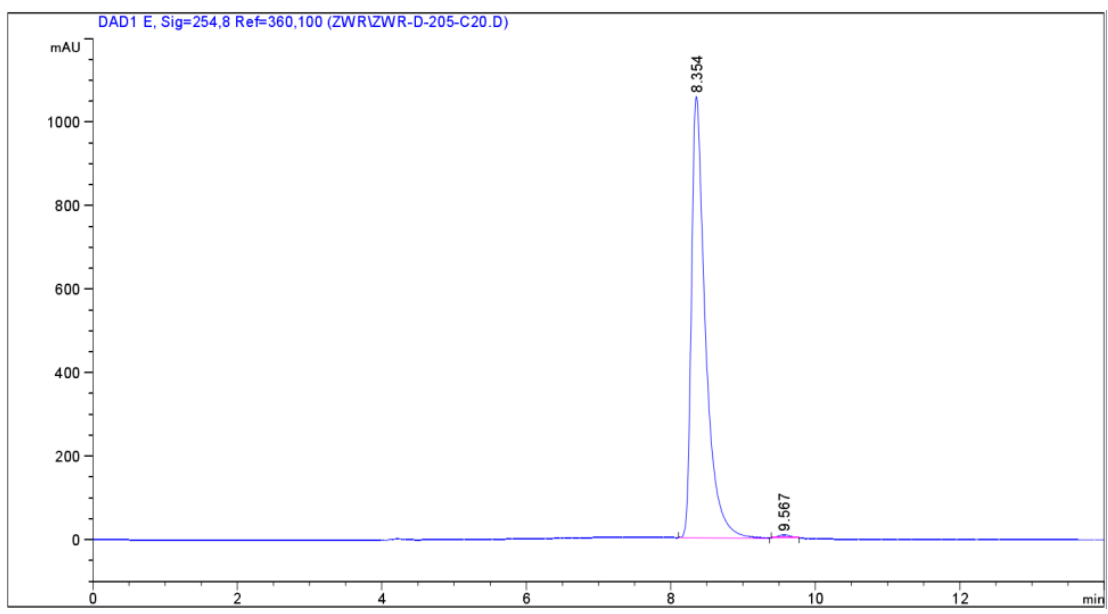

信号 1: DAD1 E， Sig=254,8 Ref=360,100

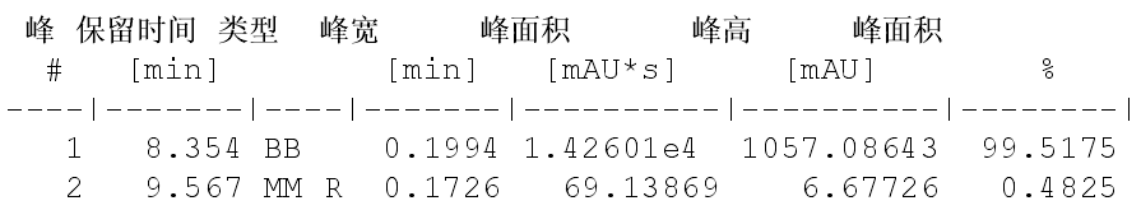




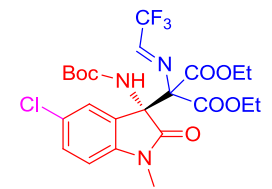

$8 c$

\section{HPLC spectrum of the racemate}

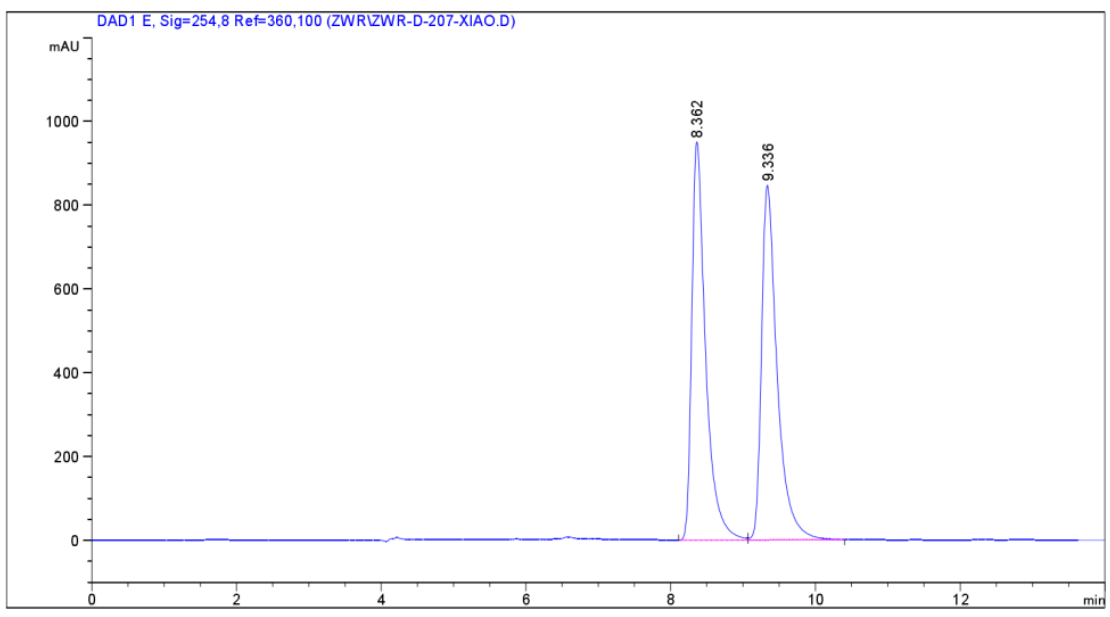

信号 1: DAD1 E， Sig=254, 8 Ref $=360,100$

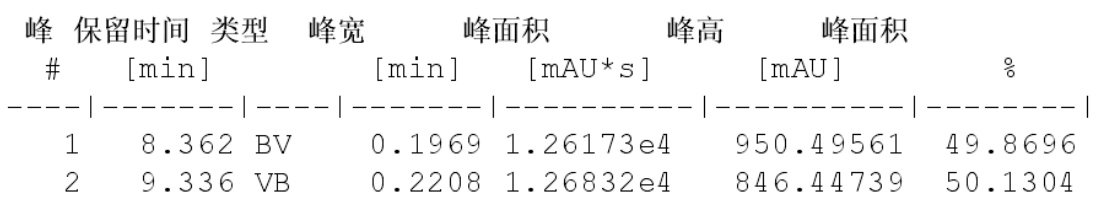

\section{HPLC spectrum of the chiral compound}

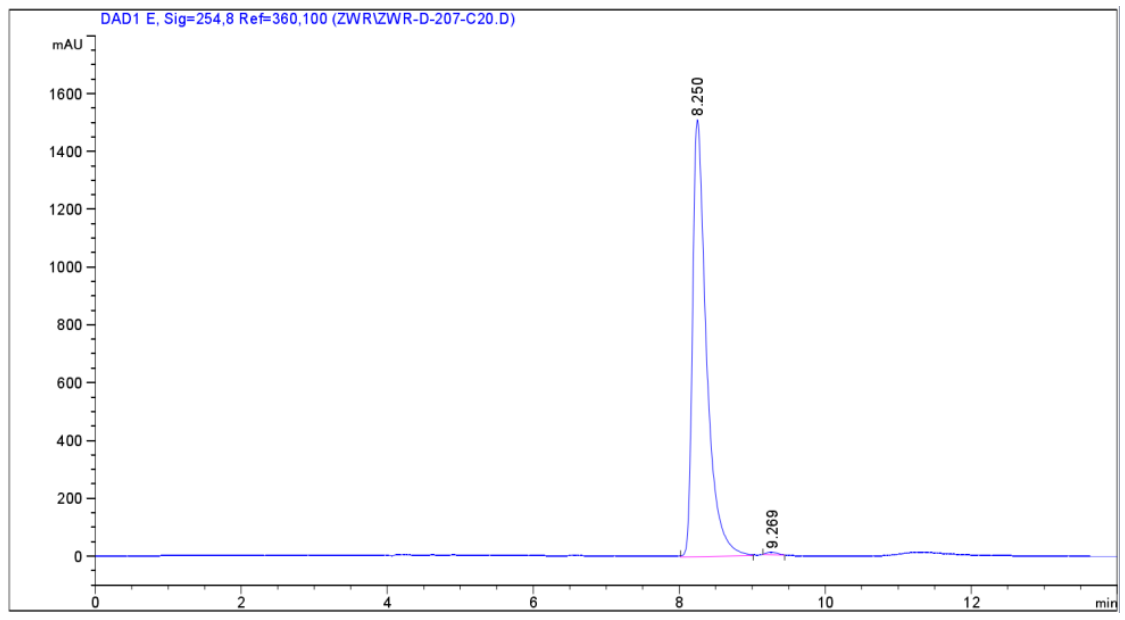

信号 1: DAD1 E， Sig=254,8 Ref $=360,100$

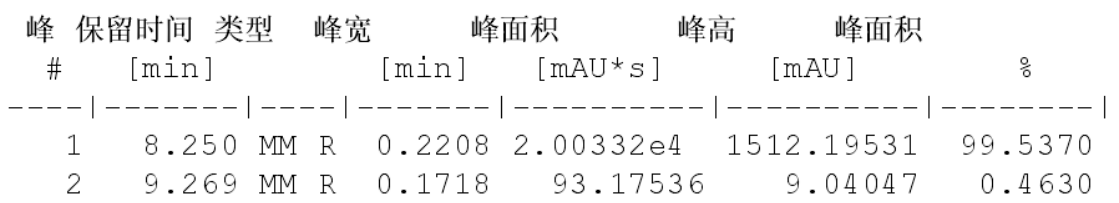




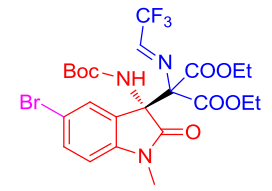

$8 d$

\section{HPLC spectrum of the racemate}

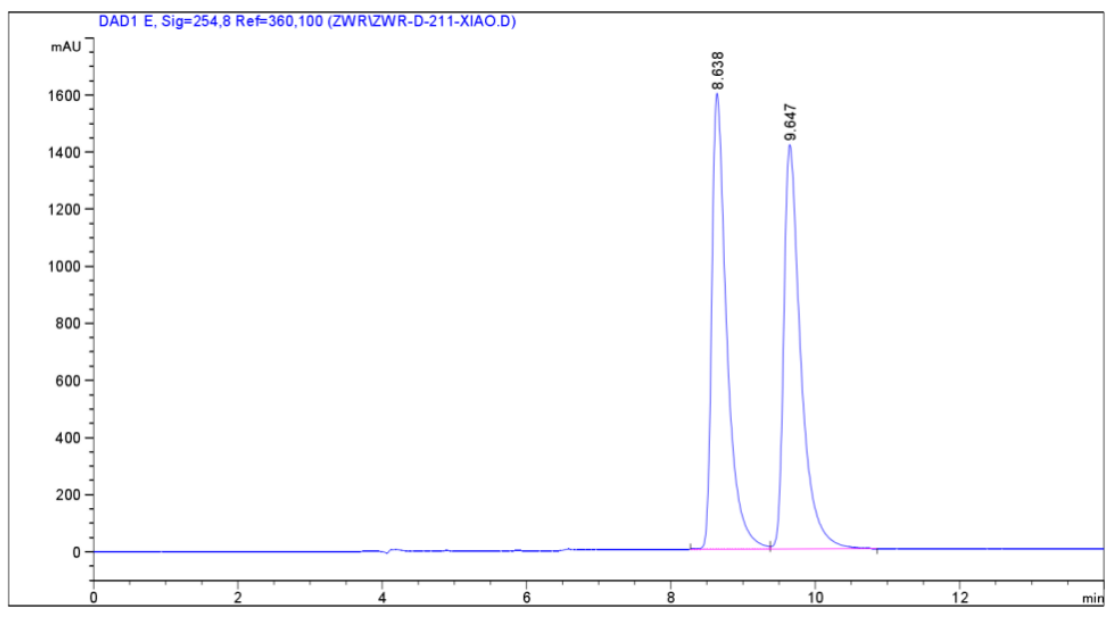

信号 1: DAD1 E， Sig=254,8 Ref=360,100

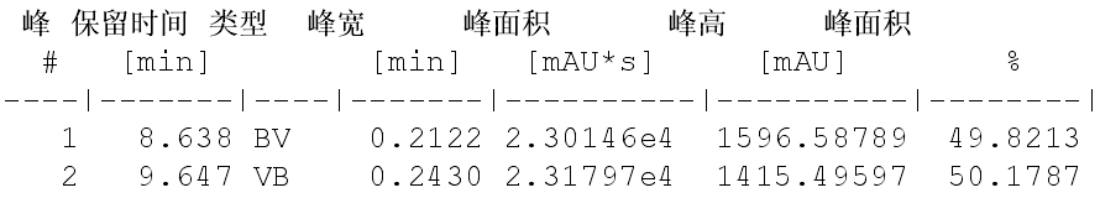

\section{HPLC spectrum of the chiral compound}

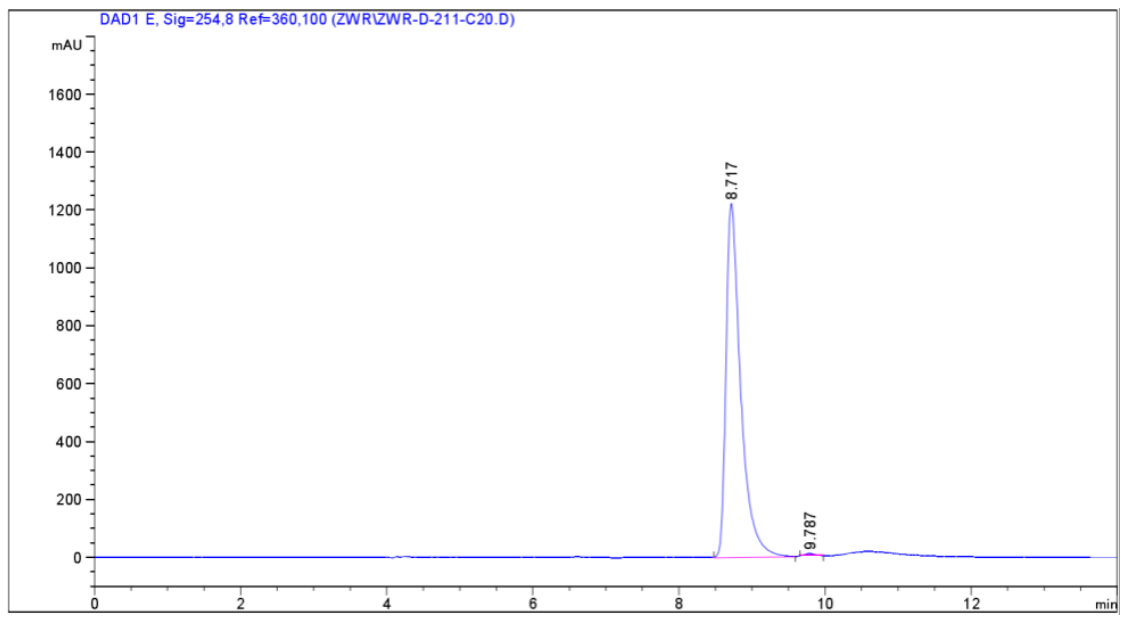

信号 1: DAD1 E, Sig=254, 8 Ref=360,100

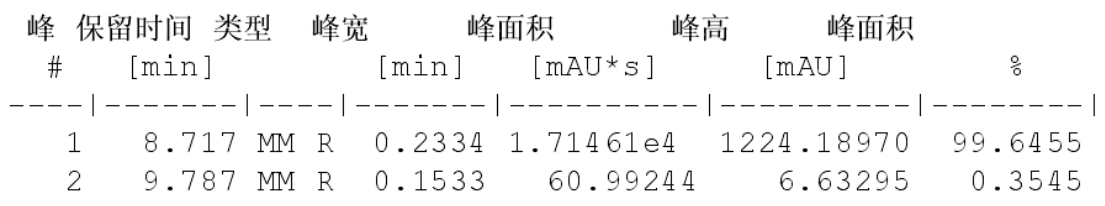




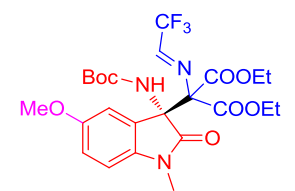

$8 \mathrm{e}$

\section{HPLC spectrum of the racemate}

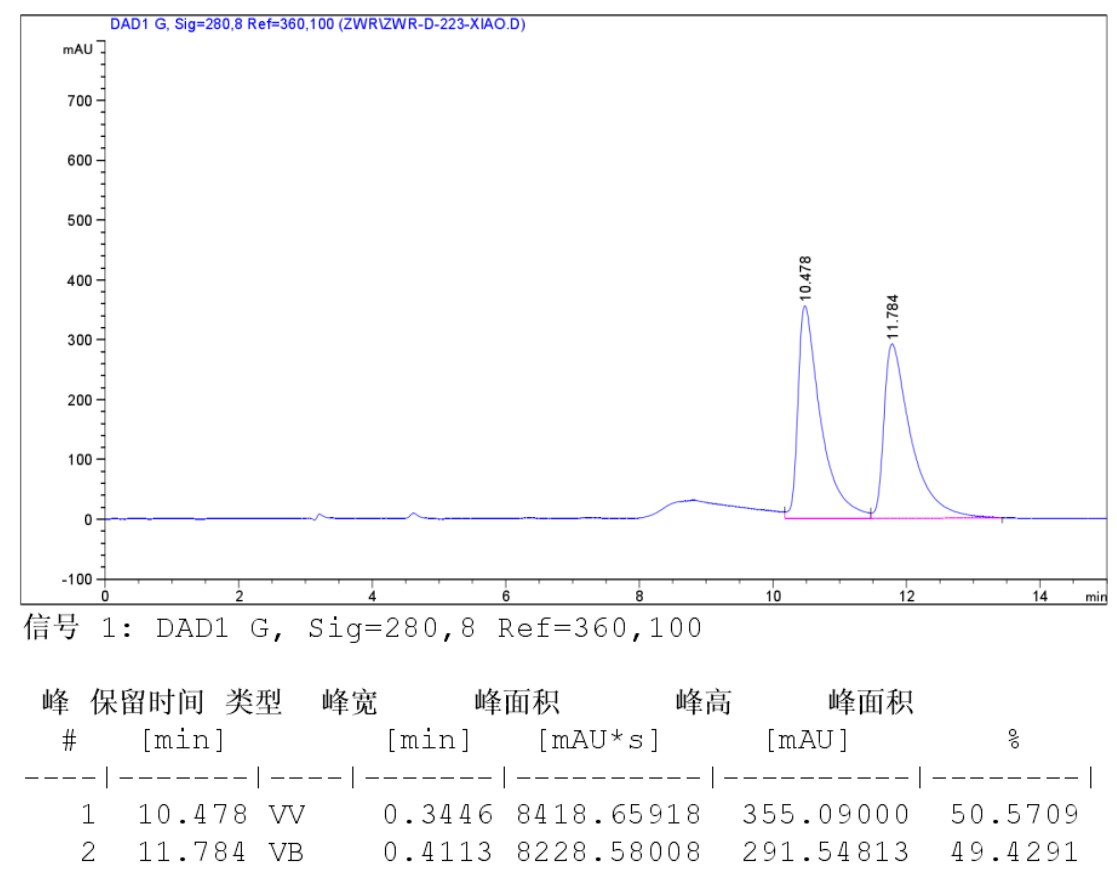

\section{HPLC spectrum of the chiral compound}

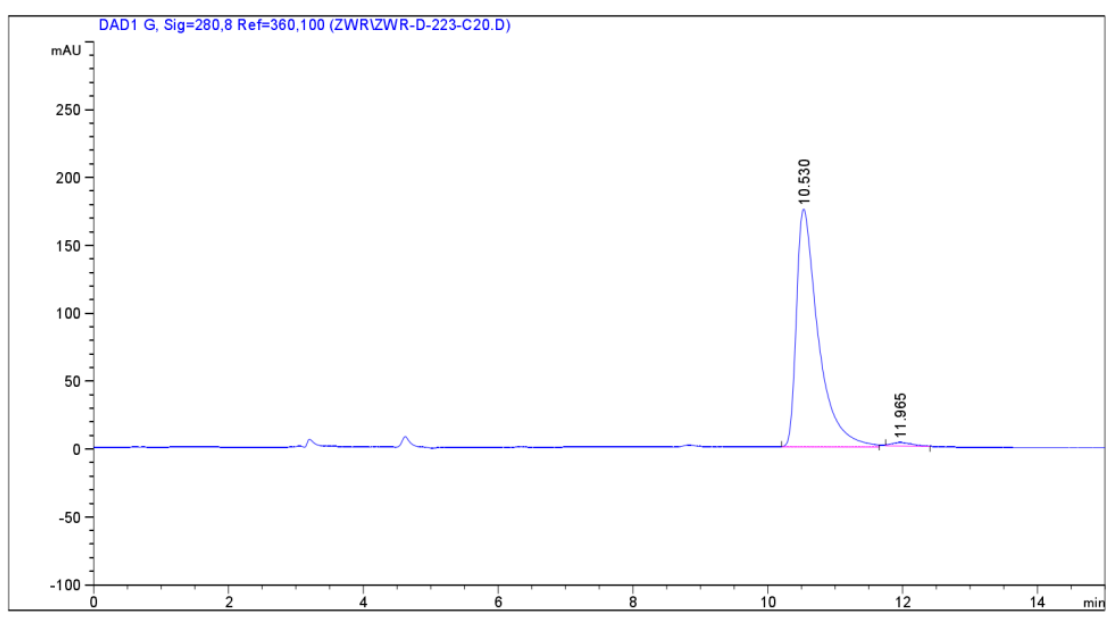

信号 1: DAD1 G, Sig=280,8 $\operatorname{Ref}=360,100$

\begin{tabular}{|c|c|c|c|c|c|c|c|}
\hline \multirow{2}{*}{$\begin{array}{c}\text { 峰 } \\
\text { \# }\end{array}$} & \multirow{2}{*}{$\begin{array}{c}\text { 保留时间 类型 } \\
\text { [min] }\end{array}$} & \multirow{2}{*}{ 峰宽 } & \multicolumn{2}{|c|}{ 峰面积 } & 峰高 & 峰面积 & \multirow[b]{2}{*}{$\%$} \\
\hline & & & [min ] & {$\left[\mathrm{mAU}{ }^{\star} \mathrm{S}\right]$} & & [mAU ] & \\
\hline & & & ----- & $-\mid-------$ & & --------- & \\
\hline 1 & $10.530 \mathrm{BE}$ & & 0.3309 & 93946.3813 & & 175.125 & 98.6639 \\
\hline 2 & $11.965 \mathrm{MI}$ & $\mathrm{R}$ & 0.3555 & 53.441 & & 2.50525 & 1.3361 \\
\hline
\end{tabular}




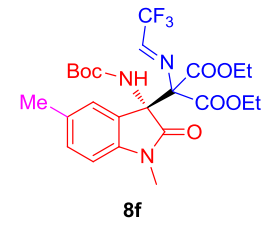

\section{HPLC spectrum of the racemate}

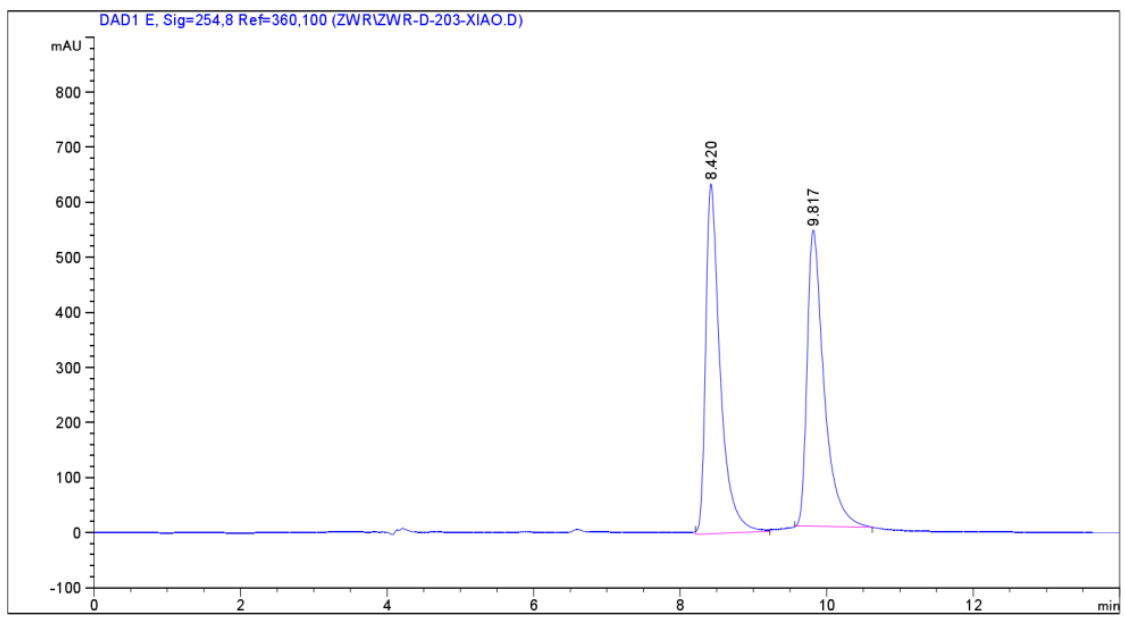

信号 1: DAD1 E，Sig=254,8 Ref=360,100

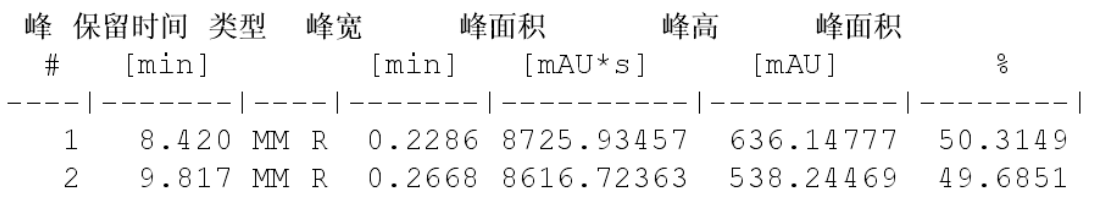

\section{HPLC spectrum of the chiral compound}

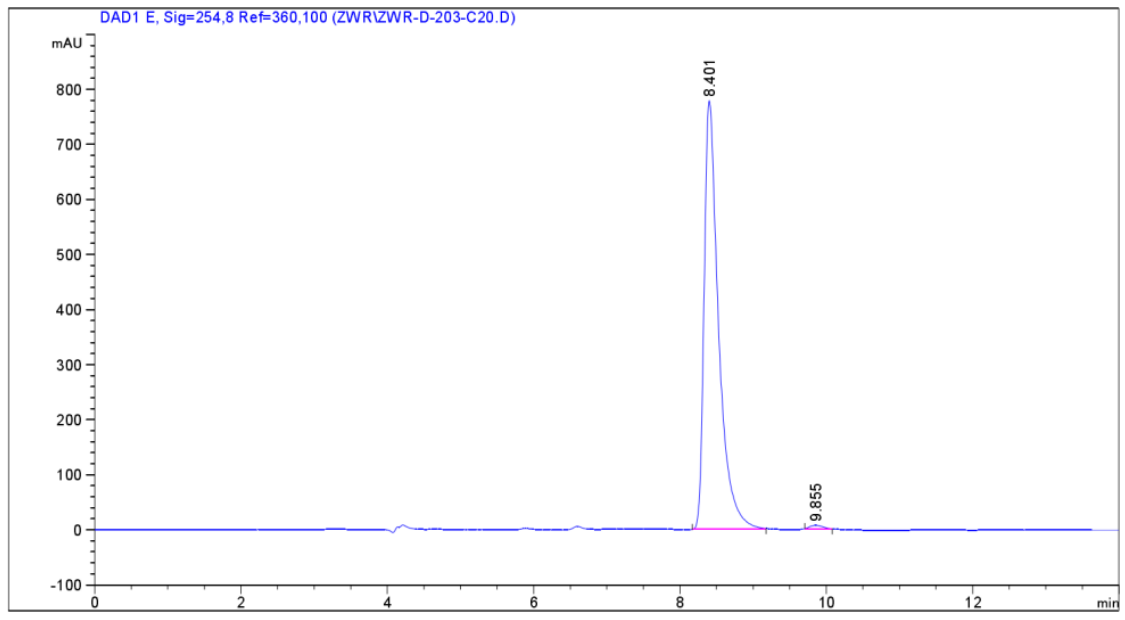

信号 1: DAD1 E， Sig=254,8 Ref $=360,100$

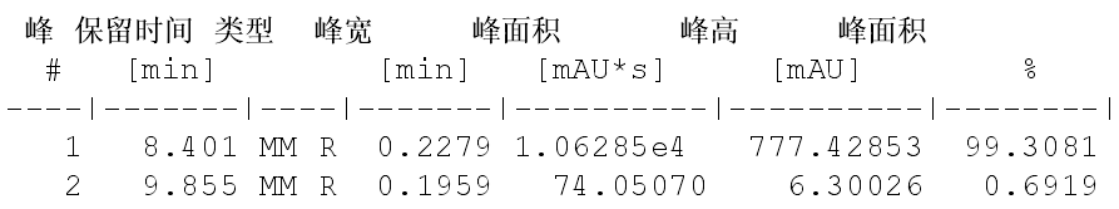




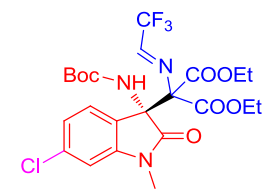

$8 \mathrm{~g}$

HPLC spectrum of the racemate

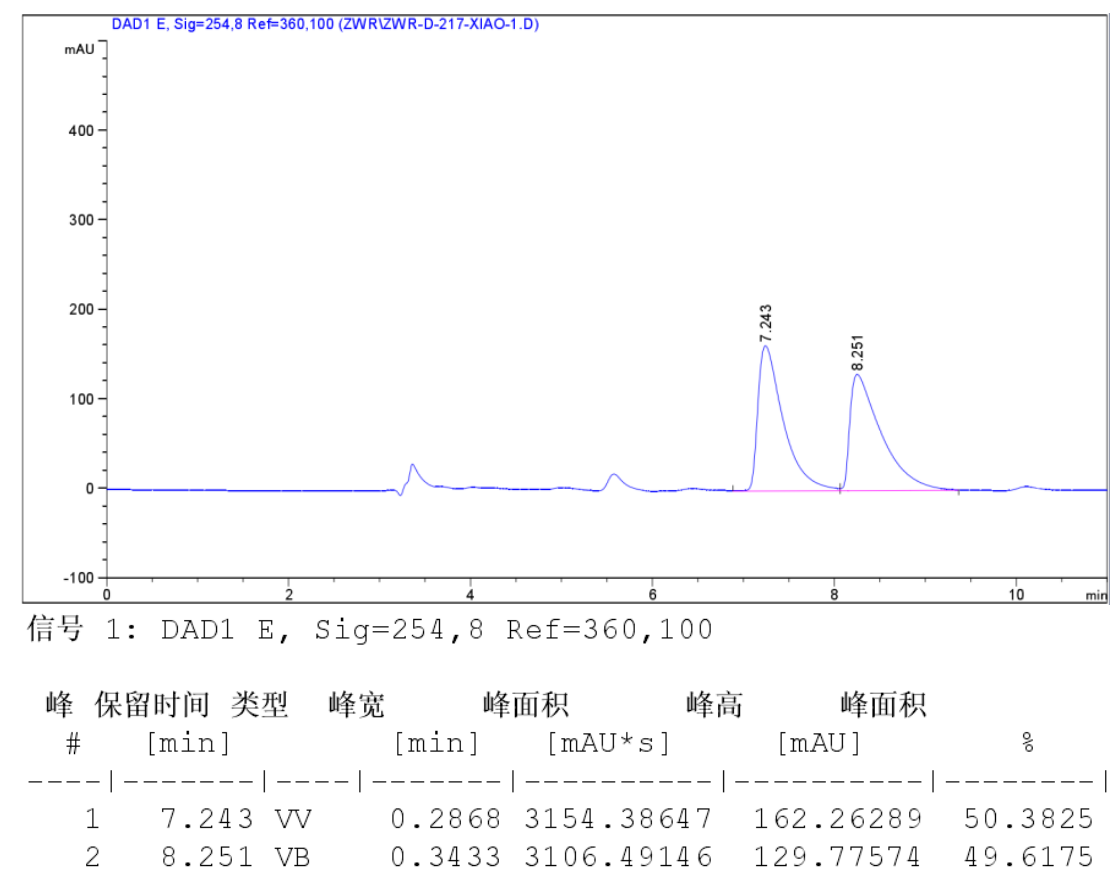

\section{HPLC spectrum of the chiral compound}

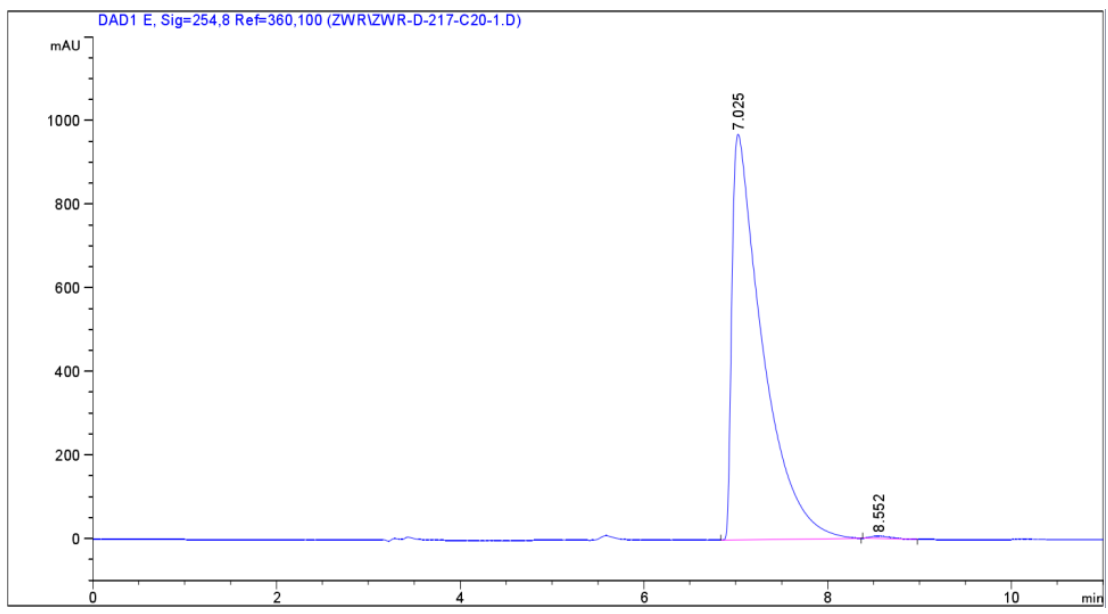

信号 1: DAD1 E， Sig=254, 8 Ref $=360,100$

\begin{tabular}{cccccc} 
峰 保留时间 类型 & 峰宽 & \multicolumn{2}{c}{ 峰面积 } & 峰高 & \multicolumn{2}{c}{ 峰面积 } \\
\# \\
{$[\mathrm{min}]$}
\end{tabular}




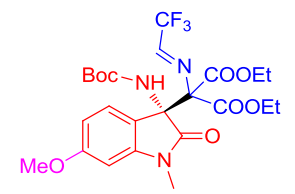

$8 \mathrm{~h}$

\section{HPLC spectrum of the racemate}

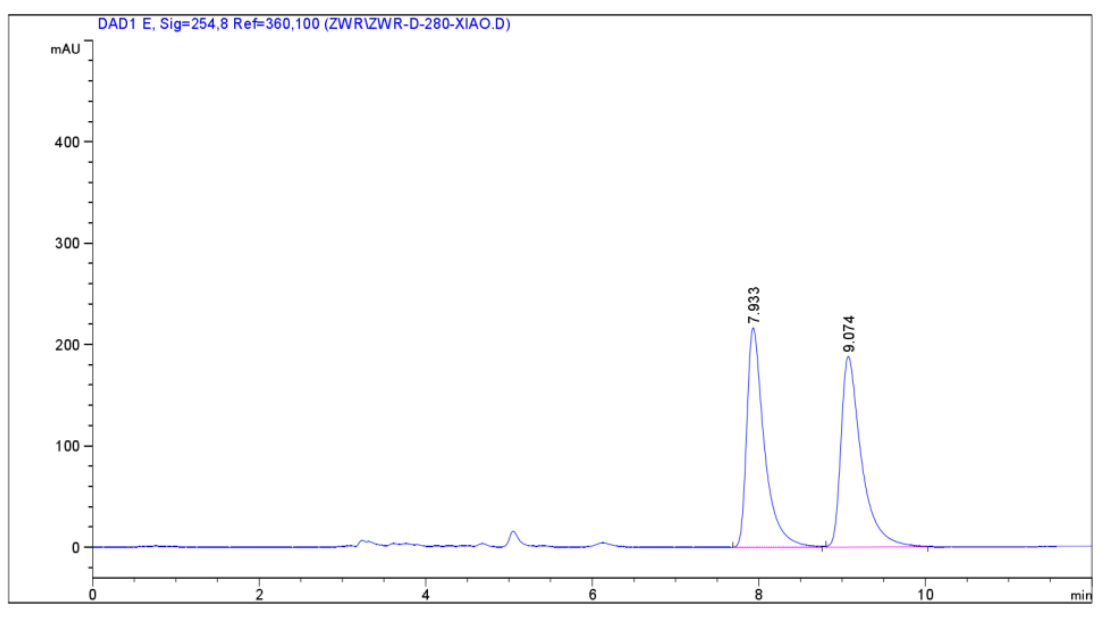

信号 1: DAD1 E, Sig=254,8 Ref=360,100

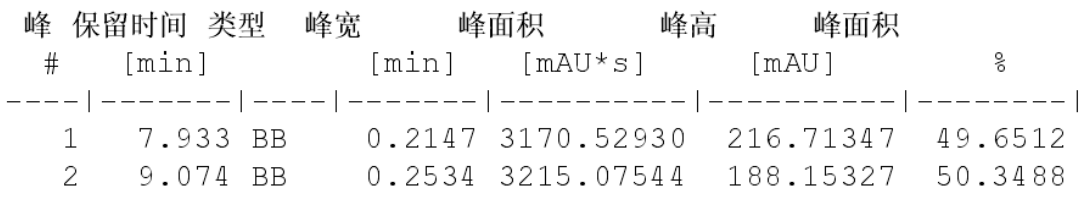

\section{HPLC spectrum of the chiral compound}

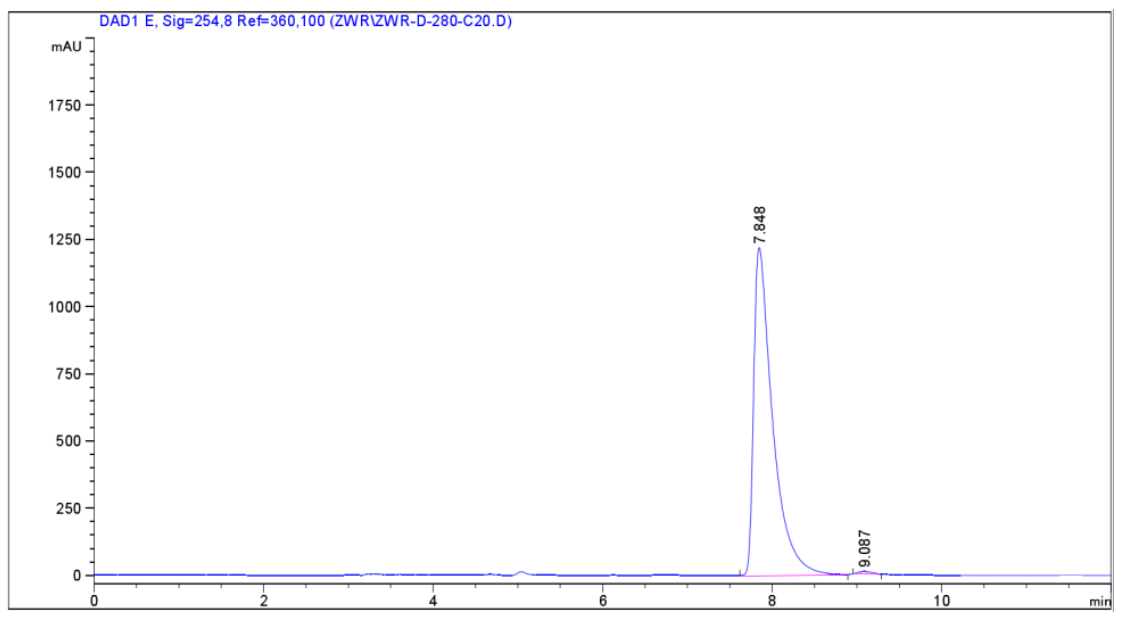

信号 1: DAD1 E, Sig $=254,8$ Ref $=360,100$

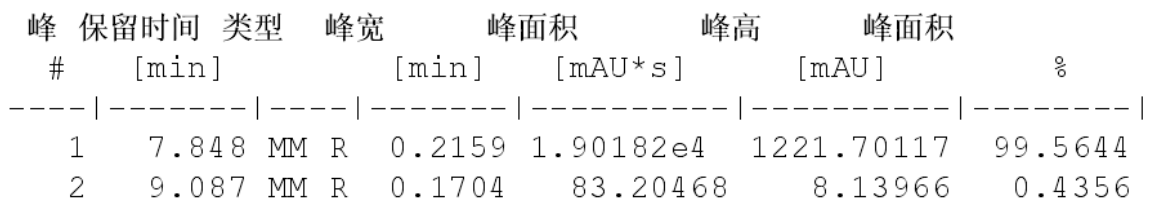




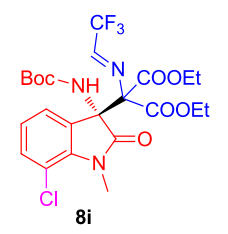

\section{HPLC spectrum of the racemate}

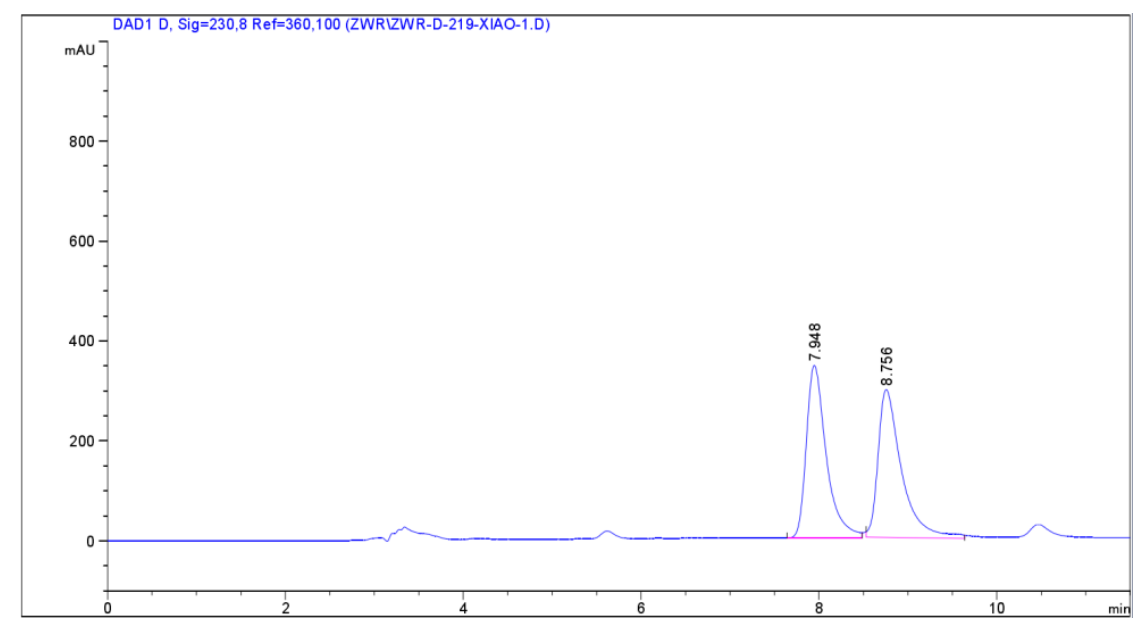

信号 1: DAD1 D, Sig $=230,8$ Ref $=360,100$

\begin{tabular}{|c|c|c|c|c|c|c|c|}
\hline 峰 & 保留时间 类型 & 峰宽 & & 峰面积 & 峰高 & \multicolumn{2}{|l|}{ 峰面积 } \\
\hline \# & {$[\mathrm{min}]$} & & [min $]$ & {$\left[\mathrm{mAU}{ }^{*} \mathrm{~s}\right]$} & & {$[\mathrm{mAU}]$} & $\frac{\circ}{0}$ \\
\hline & $---5+2+$ & & - & ---- & 1 & ------ & ------- \\
\hline & 7.948 & & 0.2359 & $9 \quad 5391.134$ & & 345.73013 & 49.7423 \\
\hline & $8.756 \mathrm{MI}$ & $\mathrm{R}$ & 0.3066 & $6 \quad 5446.992$ & 19 & 296.08496 & 50.2577 \\
\hline
\end{tabular}

\section{HPLC spectrum of the chiral compound}

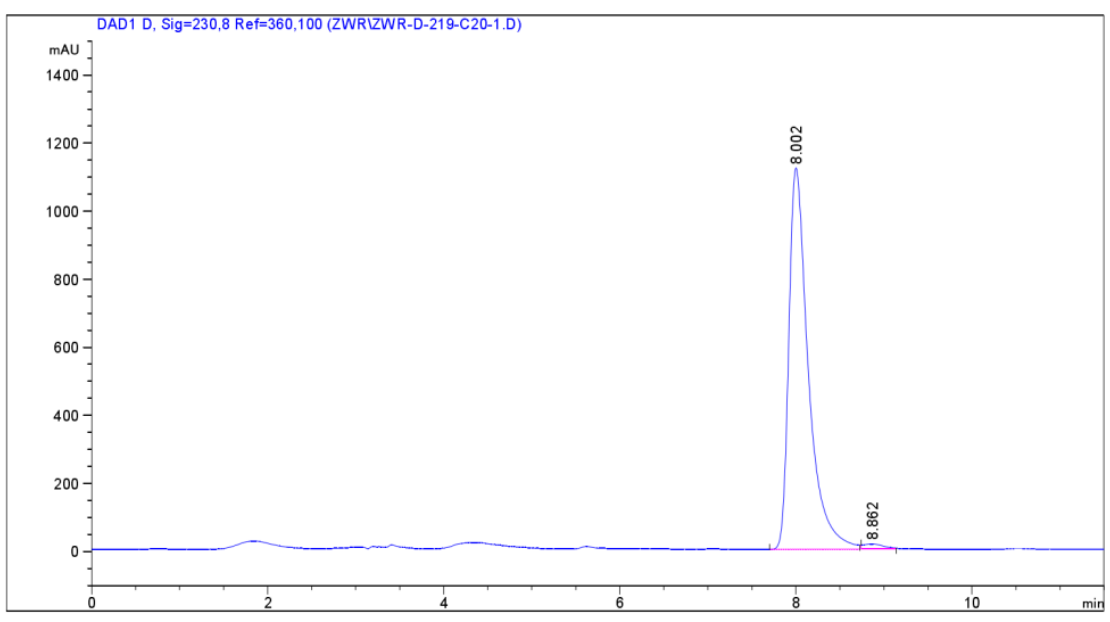

信号 1: DAD1 D， Sig=230,8 $\operatorname{Ref}=360,100$

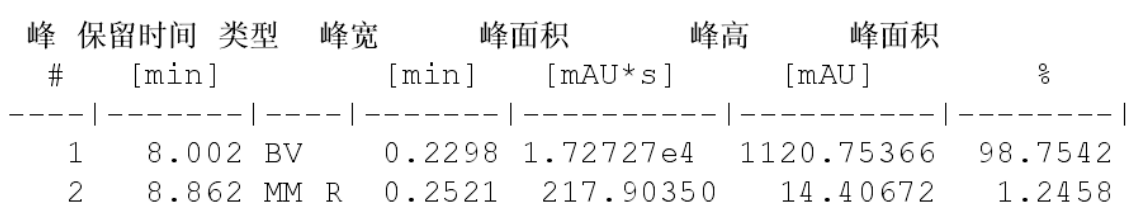




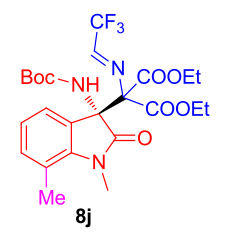

\section{HPLC spectrum of the racemate}

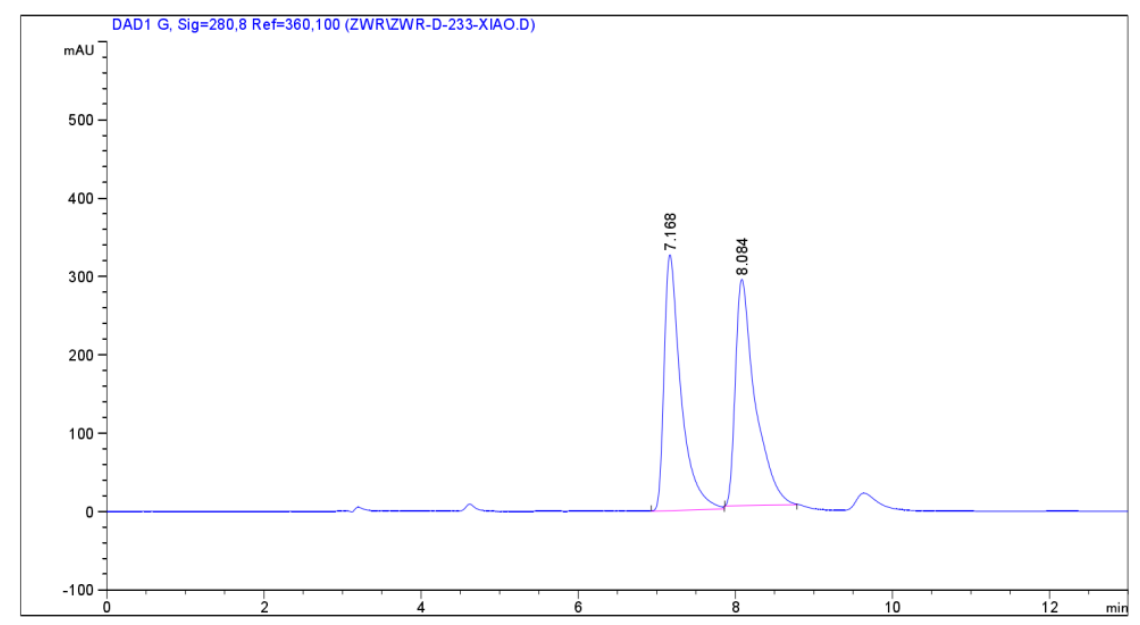

信号 1: DAD1 G， Sig $=280,8$ Ref $=360,100$

\begin{tabular}{|c|c|c|c|c|c|c|c|}
\hline 峰 & 保留时间 类型 & 峰宽 & & 峰面积 & 峰高 & 峰面积 & \\
\hline \# & [min $]$ & & [min ] & {$\left[\mathrm{mAU}^{*} \mathrm{~S}\right]$} & & {$[\mathrm{mAU}]$} & 음 \\
\hline & & & ----- & -- & & -- & --- \\
\hline & 168 & $\mathrm{R}$ & 0.2491 & 14886.453 & & 326.88303 & 2407 \\
\hline & $8.084 \mathrm{MI}$ & $\mathrm{R}$ & 0.2905 & 55037.146 & 97 & 288.97311 & 50.7593 \\
\hline
\end{tabular}

\section{HPLC spectrum of the chiral compound}

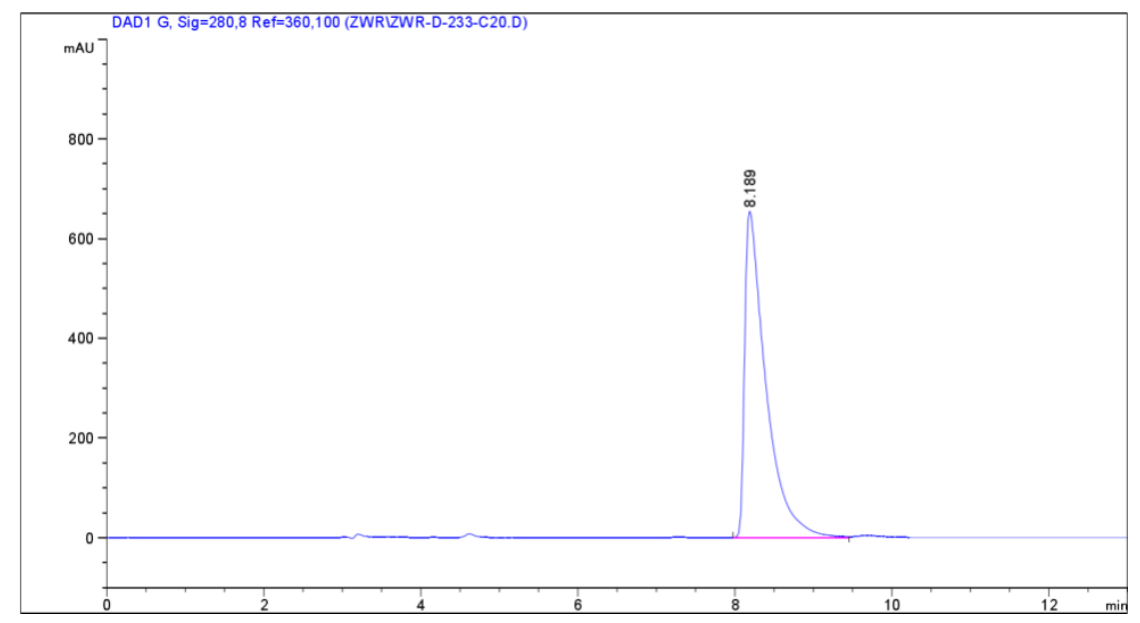

信号 1: DAD1 G， Sig $=280,8$ Ref $=360,100$

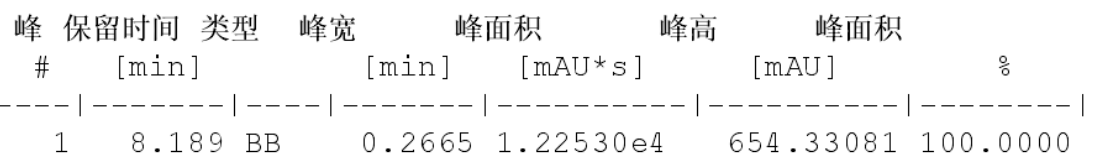




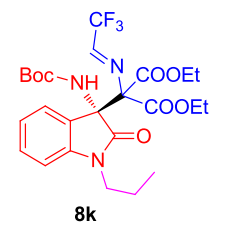

HPLC spectrum of the racemate

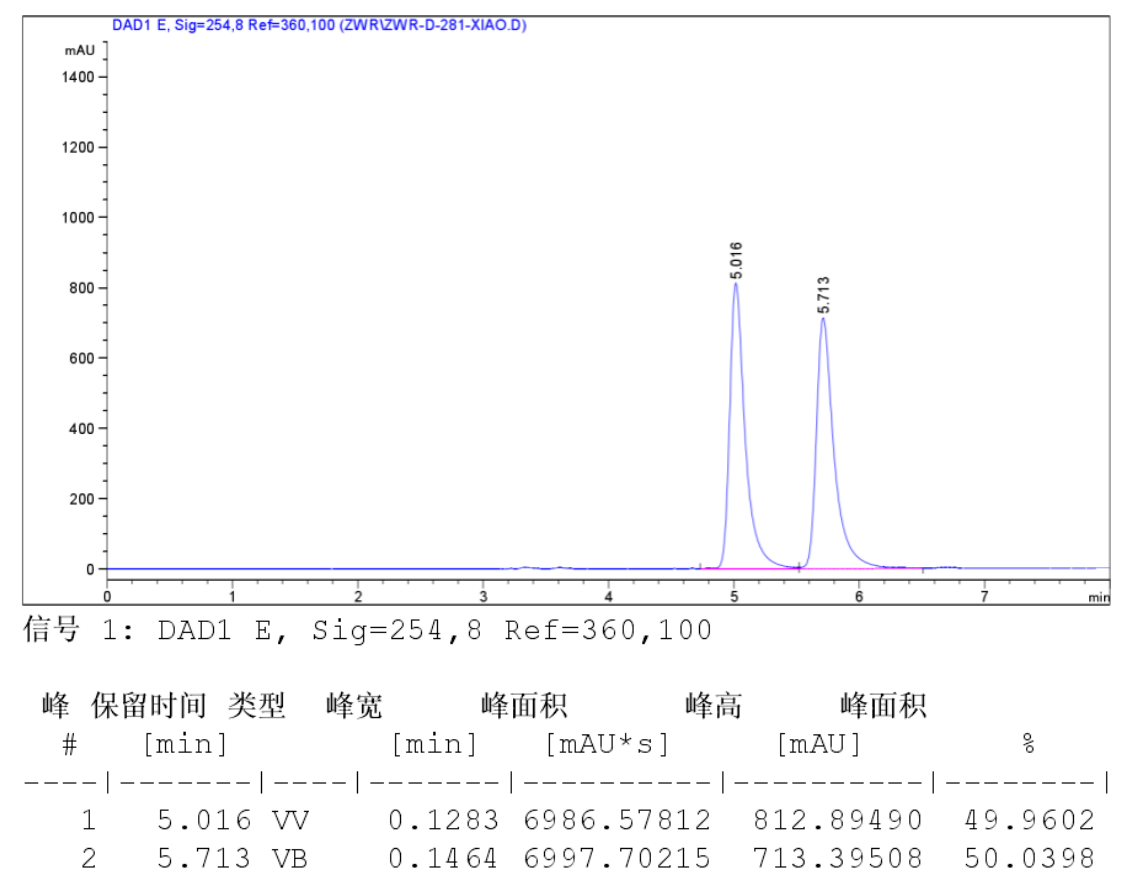

\section{HPLC spectrum of the chiral compound}

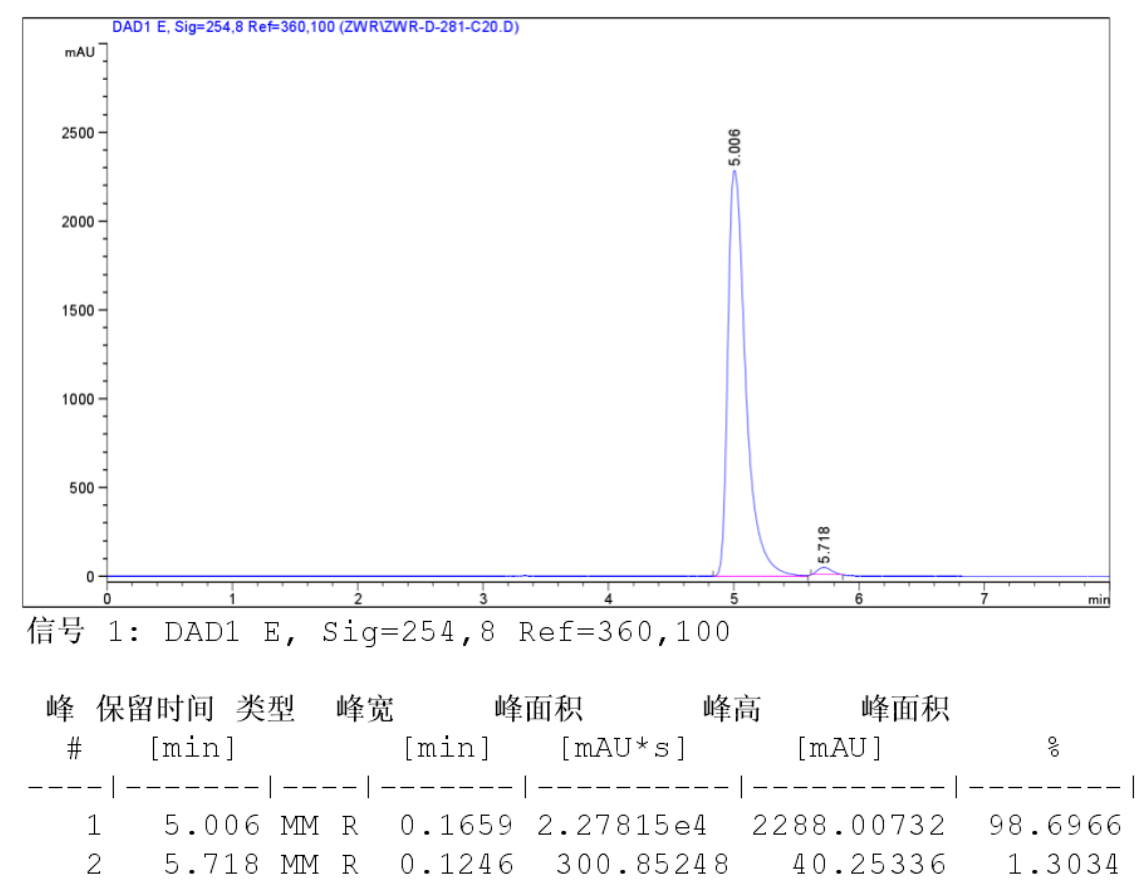




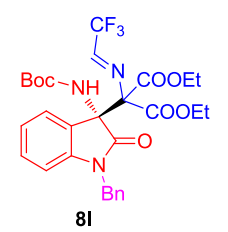

HPLC spectrum of the racemate

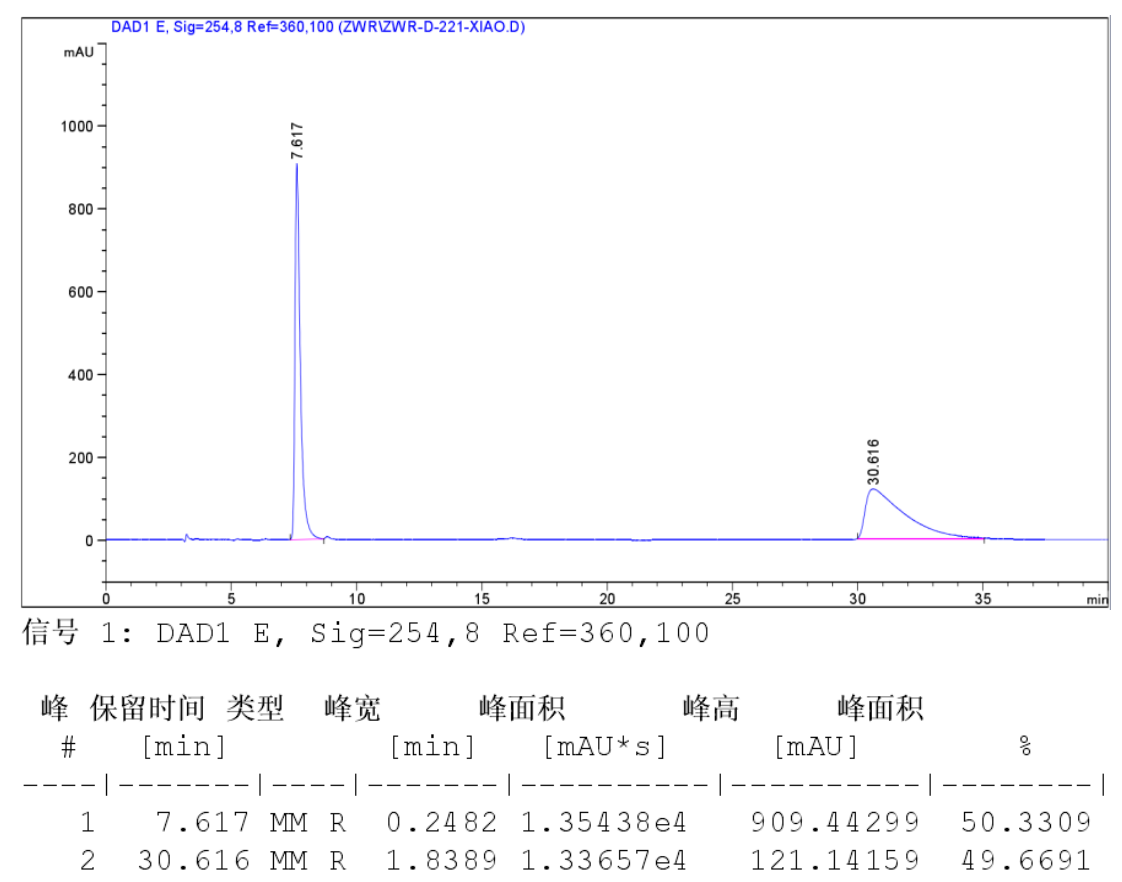

\section{HPLC spectrum of the chiral compound}

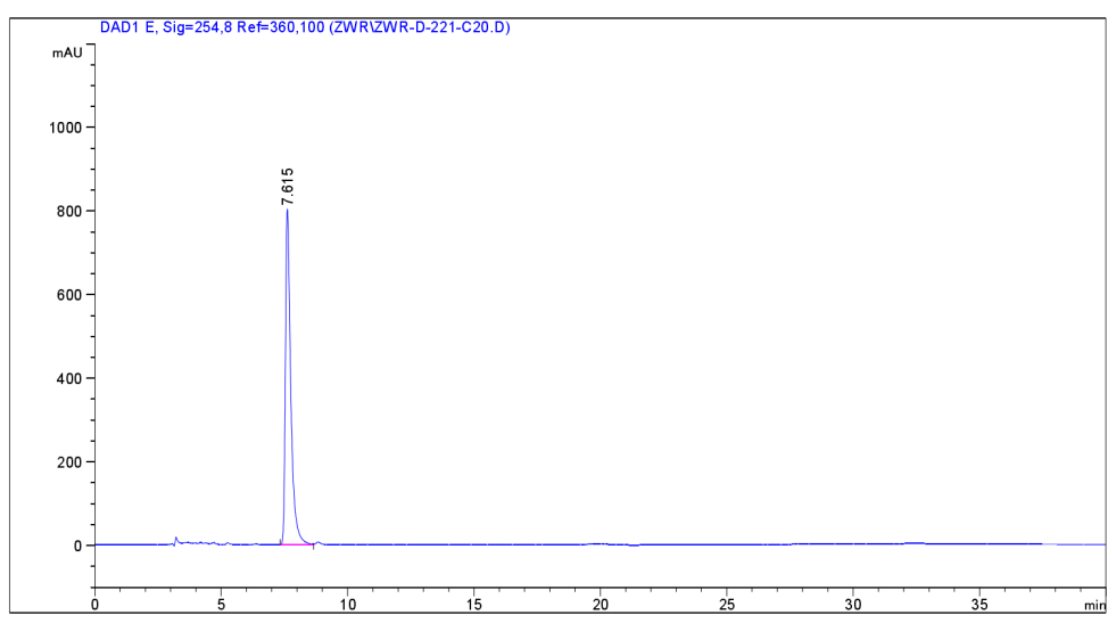

信号 1: DAD1 E， Sig=254, 8 Ref=360,100

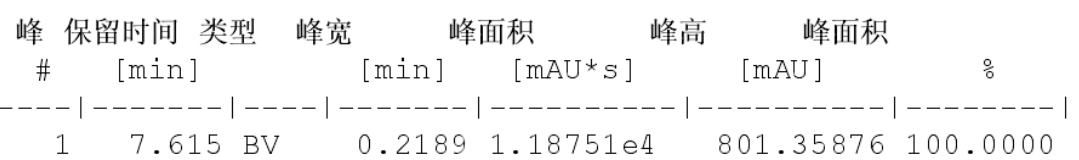




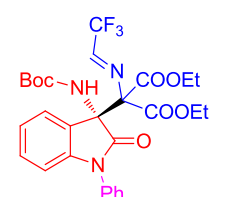

$8 \mathrm{~m}$

\section{HPLC spectrum of the racemate}

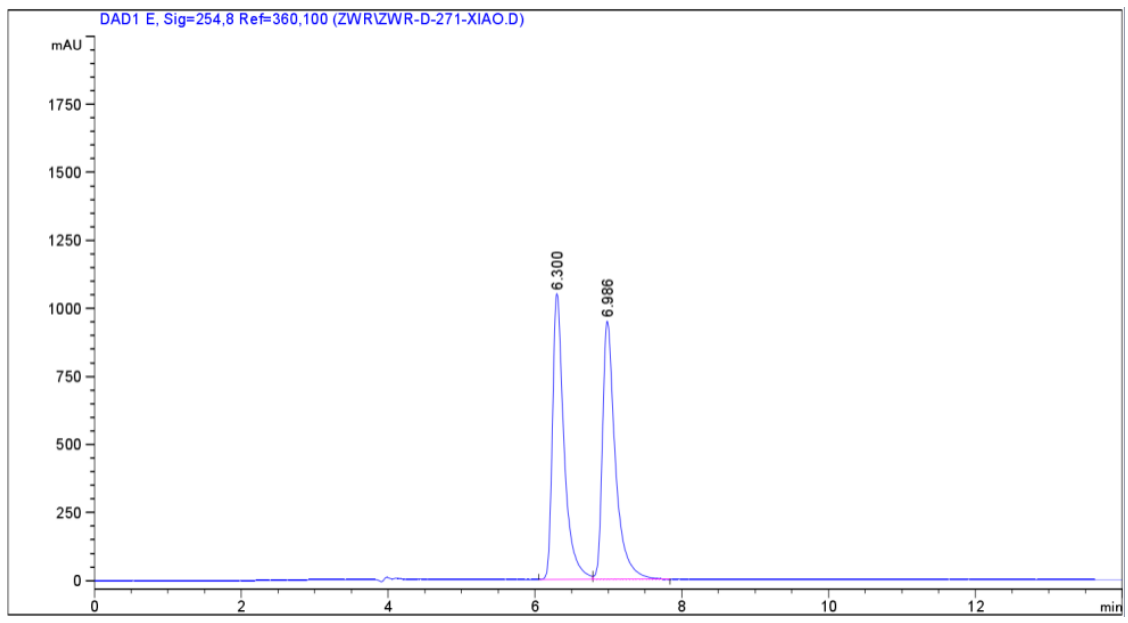

信号 1: DAD1 E， Sig=254,8 Ref=360,100

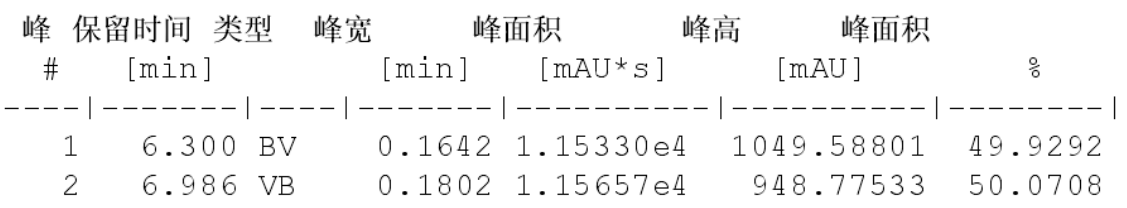

\section{HPLC spectrum of the chiral compound}

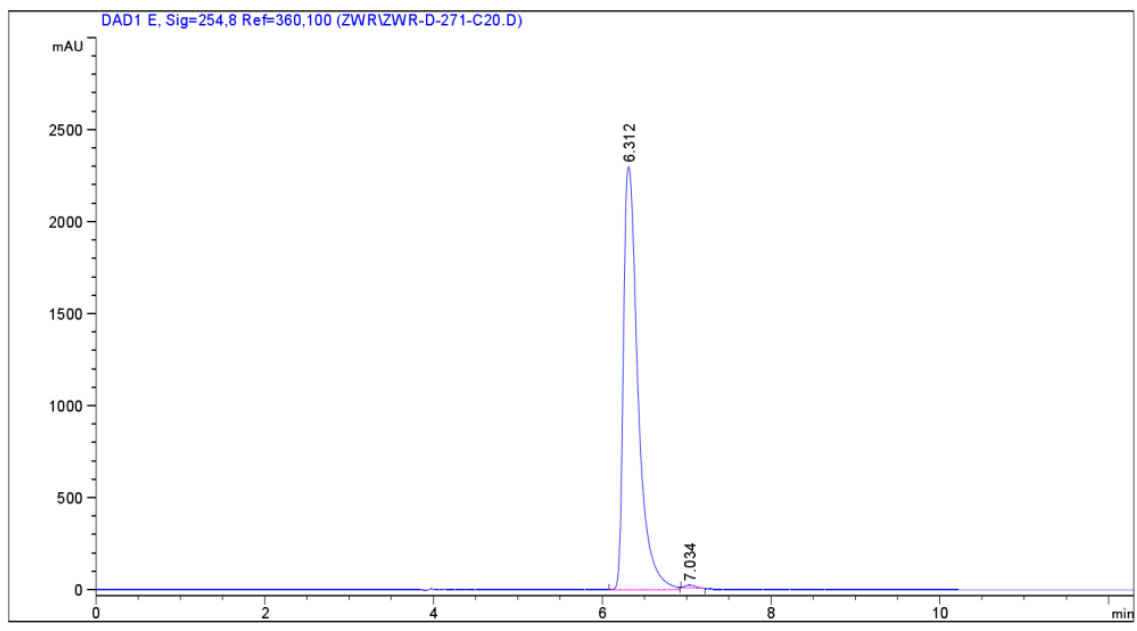

信号 1: DAD1 E， Sig=254,8 Ref=360,100

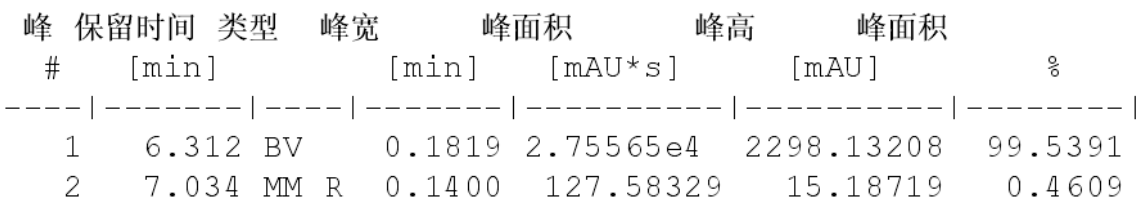




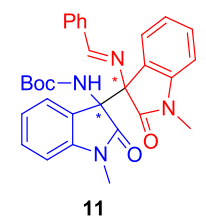

HPLC spectrum of the racemate

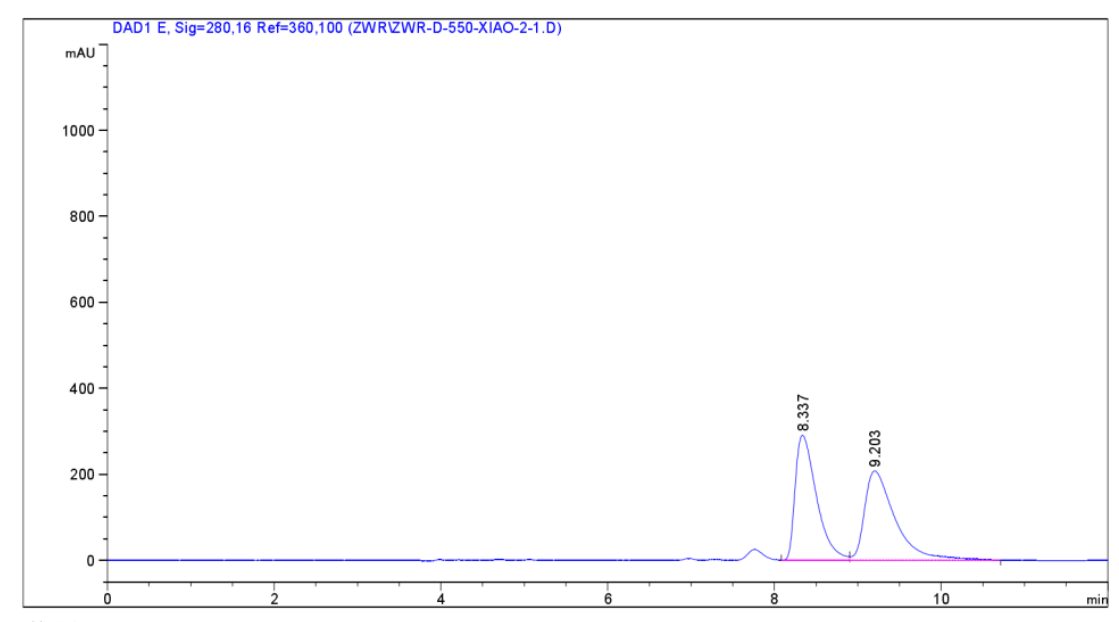

信号 1: DAD1 E， Sig=280,16 $\operatorname{Ref}=360,100$

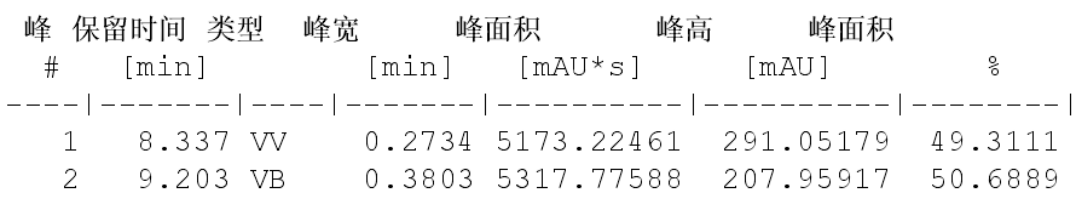

\section{HPLC spectrum of the chiral compound}

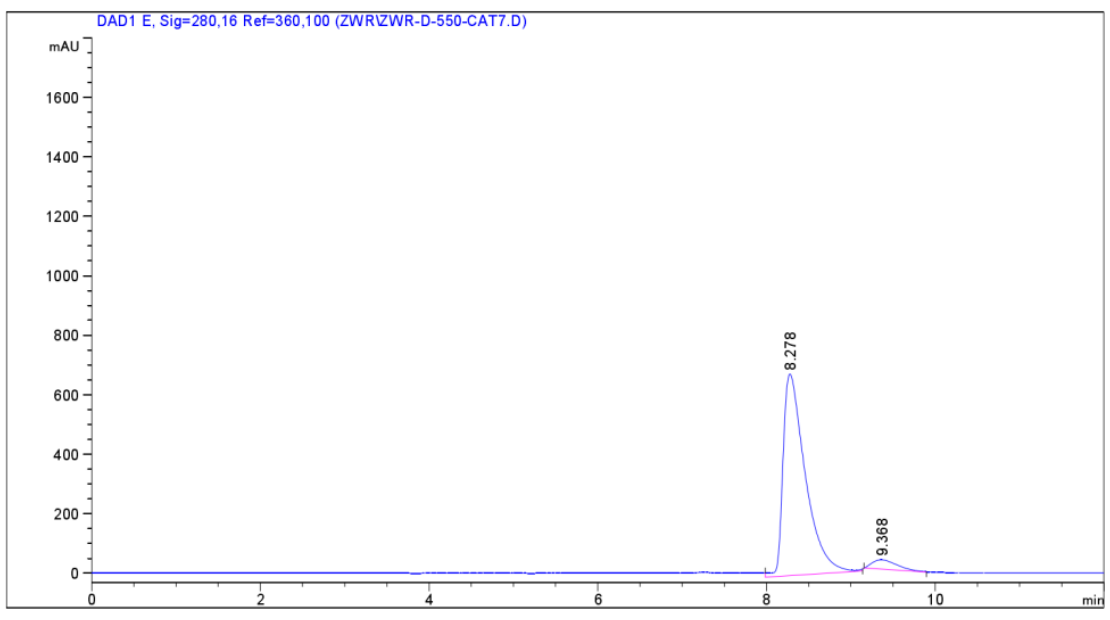

信号 1: DAD1 E, Sig=280,16 Ref $=360,100$

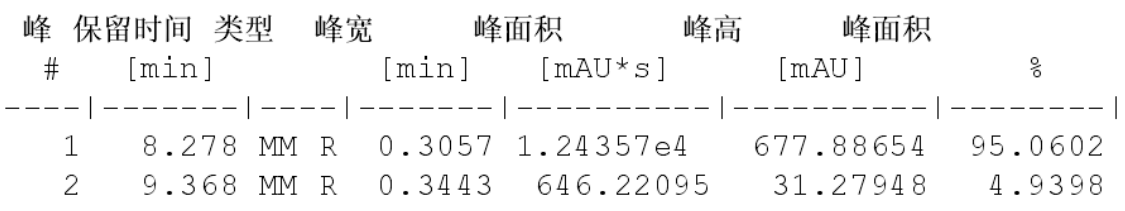




\section{References}

[1] a) Ma, M.-X.; Zhu, Y.-Y.; Sun, Q.-T.; Li, X.-Y.; Su, J.-H.; Zhao, L.; Zhao, Y.-Y.; Qiu, S.; Yan, W.-J.; Wang, K.-Y.; Wang, R. Chem. Commun. 2015, 51, 8789-8792. b) Su, J.; Ma, Z.; Li, X.; Lin, L.; Shen, Z.; Yang, P.; Li, Y.; Wang, H.; Yan, W.; Wang, K.; Wang, R. Adv. Synth. Catal. 2016, 358, 3777-3785. c) Zhao, H.-W.; Li, B.; Pang, H.-L.; Tian, T.; Chen, X.-Q.; Song, X.-Q.; Meng, W.; Yang, Z.; Zhao, Y.-D.; Liu, Y.-Y. Org. Lett. 2016, 18, 848-851.

[2] a) Storer, R. I.; Aciro, C.; Jones, L. H. Chem. Soc. Rev. 2011, 40, 2330-2346. b) Lin, N.; Long, X. W.; Chen, Q.; Zhu, W. R.; Wang, B. C.; Chen, K. B.; Jiang, C. W.; Weng, J.; Lu, G. Tetrahedron 2018, 74, 3734-3741. c) Zhu, W. R.; Chen, Q.; Lin, N.; Chen, K. B.; Zhang, Z. W.; Fang, G.; Weng, J.; Lu, G. Org. Chem. Front. 2018, 5, 1375-1380.

[3] Frisch, M. J.; Trucks, G. W.; Schlegel, H. B.; Scuseria, G. E.; Robb, M. A.; Cheeseman, J. R.; Scalmani, G.; Barone, V.; Petersson, G. A.; Nakatsuji, H.; Li, X.; Caricato, M.; Marenich, A. V.; Bloino, J.; Janesko, B. G.; Gomperts, R.; Mennucci, B.; Hratchian, H. P.; Ortiz, J. V.; Izmaylov, A. F.; Sonnenberg, J. L.; Williams, Ding, F.; Lipparini, F.; Egidi, F.; Goings, J.; Peng, B.; Petrone, A.; Henderson, T.; Ranasinghe, D.; Zakrzewski, V. G.; Gao, J.; Rega, N.; Zheng, G.; Liang, W.; Hada, M.; Ehara, M.; Toyota, K.; Fukuda, R.; Hasegawa, J.; Ishida, M.; Nakajima, T.; Honda, Y.; Kitao, O.; Nakai, H.; Vreven, T.; Throssell, K.; Montgomery, J. A.; Peralta, J. E.; Ogliaro, F.; Bearpark, M. J.; Heyd, J. J.; Brothers, E. N.; Kudin, K. N.; Staroverov, V. N.; Keith, T. A.; Kobayashi, R.; Normand, J.; Raghavachari, K.; Rendell, A. P.; Burant, J. C.; lyengar, S. S.; Tomasi, J.; Cossi, M.; Millam, J. M.; Klene, M.; Adamo, C.; Cammi, R.; Ochterski, J. W.; Martin, R. L.; Morokuma, K.; Farkas, O.; Foresman, J. B.; Fox, D. J. Gaussian 16 Rev. B.01, Wallingford, CT, (2016).

[4] Zhao, Y.; Truhlar, D. G. Acc. Chem. Res. 2008, 41, 157-167.

[5] Marenich, A. V.; Cramer, C. J.; Truhlar, D. G. J. Phys. Chem. B. 2009, 113, 6378-6396. 